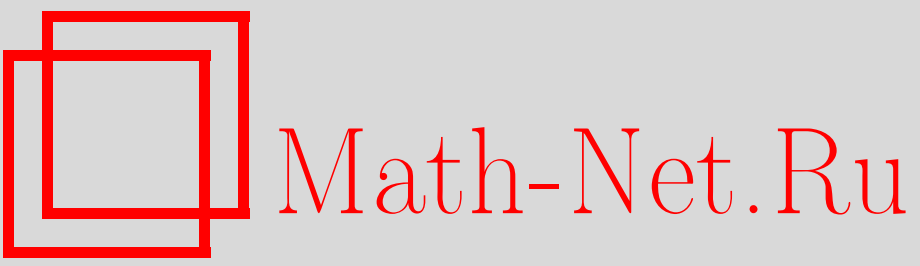

С. И. Пискарев, А. В. Овчинников, Аттракторы, затенение и аппроксимация абстрактных полулинейных дифференциальных уравнений, Итоги науки и техн. Сер. Соврем. мат. и ее прил. Темат. обз., 2021, том 189, 3-130

DOI: https://doi.org/10.36535/0233-6723-2021-189-3-130

Использование Общероссийского математического портала Math-Net.Ru подразумевает, что вы прочитали и согласны с пользовательским соглашением

http://www.mathnet.ru/rus/agreement

Параметры загрузки:

IP : 54.210 .77 .194

26 апреля 2023 г., 16:51:03 


\title{
АТТРАКТОРЫ, ЗАТЕНЕНИЕ И АППРОКСИМАЦИЯ АБСТРАКТНЫХ ПОЛУЛИНЕЙНЫХ ДИФФЕРЕНЦИАЛЬНЫХ УРАВНЕНИЙ
}

\author{
(c) 2021 г. $\quad$ С. И. ПИСКАРЕВ, А. В. ОВЧИННИКОВ
}

\begin{abstract}
АннотАция. В обзоре обсуждаются такие разделы теории аппроксимации абстрактных дифференциальных уравнений как аппроксимация аттракторов в случае гиперболических стационарных точек, затенение и аппроксимация дробных по времени полулинейных задач.

Ключевъе слова: абстрактное параболическое уравнение, общая аппроксимационная схема, компактная сходимость, аттрактор, неустойчивое многообразие, устойчивое многообразие, верхняя и нижняя полунепрерывность аттрактора, принцип родственности, принцип компактной аппроксимации, полулинейное дифференциальное уравнение в банаховом пространстве, периодическое решение полулинейного уравнения, устойчивость решения по Ляпунову, гиперболическая точка равновесия, полупоток, вращение векторного поля, индекс решения, затенение, аналитическая $C_{0}$-полугруппа, банахово пространство, полудискретизация, дискретизация по пространству, дискретизация по времени, дробное уравнение, дробная степень оператора, уплотняющий оператор.
\end{abstract}

\section{ATTRACTORS, SHADOWING, AND APPROXIMATION OF ABSTRACT SEMILINEAR DIFFERENTIAL EQUATIONS}

\author{
(c) 2021 S. I. PISKAREV, A. V. OVCHINNIKOV
}

Abstract. The review covers such sections of the theory of approximation of abstract differential equations as the approximation of attractors in the case of hyperbolic stationary points, the shadowing and, finally, the approximation of fractional in time semilinear problems.

Keywords and phrases: abstract parabolic equations, general approximation scheme, compact convergence of resolvents, attractor, unstable manifold, stable manifold, upper and lower semicontinuity of attractors, affinity principle, principle of compact approximation, semilinear differential equation, Banach space, periodic solution, Lyapunov stability, hyperbolic equilibrium, semiflow, rotation of a vector field, index of a solution, shadowing, analytic $C_{0}$-semigroup, semidiscretization, discretization in space, discretization in time, fractional equation, fractional power of an operator, condensing operator.

AMS Subject Classification: 34D09, 34G20, 35B41, 35K55, 35K58, 35K90, 35R11, 47J35, 65J08

Работа выполнена при поддержке Российского фонда фундаментальных исследований (проекты №o 20-1150004, 21-51-46001) и Российского научного фонда (проект № 20-11-20085, в части раздела 5). 


\section{СОДЕРЖАНИЕ}

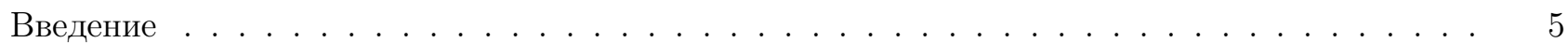

Предварительные сведения . . . . . . . . . . . . . . . . . . . . . . . . . . 6

Глава 1. Полулинейные уравнения . . . . . . . . . . . . . . . . . . . . . . . 7

1.1. Дифференциальные уравнения в банаховых пространствах . . . . . . . . . . . . . . . . 7

1.2. Некоторые интересные свойства . . . . . . . . . . . . . . . . . . . . . . . . . 8

1.3. Теорема АВC . . . . . . . . . . . . . . . . . . . . . . . . . 12

1.4. Сходимость полудискретизаций для периодической задачи . . . . . . . . . . . . . . . . 13

Глава 2. Сходимость аттракторов . . . . . . . . . . . . . . . . . . . . . . . . . . . . . . 20

2.1. Дискретная сходимость множеств . . . . . . . . . . . . . . . . . . . . . . . . . . . . . . . 21

2.2. Свойство равномерного притяжения и непрерывная сходимость . . . . . . . . . . . . . 21

2.3. Абстрактные параболические задачи . . . . . . . . . . . . . . . . . . . . . . . . . . . 23

Глава 3. Сходимость аттракторов в гиперболическом случае . . . . . . . . . . . . . . . . . 25

3.1. Аттракторы абстрактных параболических задач . . . . . . . . . . . . . . . . . . . 26

3.2. Аппроксимация операторов по общей дискретизационной схеме . . . . . . . . . . . . . 35

3.3. Аппроксимация аттракторов . . . . . . . . . . . . . . . . . . . . . . . . . . . . . . . . 39

3.4. Аппроксимация неустойчивых многообразий . . . . . . . . . . . . . . . . . . . . 43

3.5. Вспомогательные результаты . . . . . . . . . . . . . . . . . . . . . . . 45

Глава 4. Затенение для абстрактных параболических уравнений . . . . . . . . . . . . . . . 51

4.1. Предварительные сведения . . . . . . . . . . . . . . . . . . . . . . . . . . . 52

4.2. Дискретизация операторов и полугрупп . . . . . . . . . . . . . . . . . . . . . . . 55

4.3. Затенение по пространству . . . . . . . . . . . . . . . . . . . . . . . . . . . . . . . 59

4.4. Затенение по времени . . . . . . . . . . . . . . . . . . . . . . . . . . . . 65

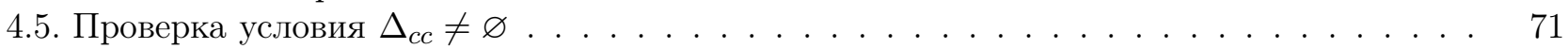

4.6. Дихотомия для полугруппп . . . . . . . . . . . . . . . . . . . . . . . . . 73

4.7. Общая дихотомия при полудискретизации . . . . . . . . . . . . . . . . . . . . . . . . . . 79

4.8. Общая дихотомия при дискретизации по временн' ой переменной . . . . . . . . . . . . 82

4.9. Полудискретная дихотомия для уплотняющих операторов . . . . . . . . . . . . . . . . 83

4.10. Приложения с уплотняющими условиями . . . . . . . . . . . . . . . . . . . . . 86

Глава 5. Полулинейные дробные уравнения . . . . . . . . . . . . . . . . . . . . . . . . . . 87

5.1. Постановка задачи . . . . . . . . . . . . . . . . . . . . . . . . 88

5.2. Полудискретная аппроксимация в специальном случае . . . . . . . . . . . . . . . . . . 89

5.3. Случай классической правой части . . . . . . . . . . . . . . . . . . . . . . . . . . . . 96

5.4. Полудискретная аппроксимация в классическом случае . . . . . . . . . . . . . . . . . . 98

5.5. Коэрцитивность в пространствах $C_{0}^{\alpha}([0, T] ; E) \ldots \ldots \ldots \ldots 1 . \ldots \ldots$

Глава 6. Устойчивые многообразия для дробных полулинейных уравнений . . . . . . . . . 106

6.1. Формулировка дробных полулинейных задач . . . . . . . . . . . . . . . . . . . . . . . . 107

6.2. Существование устойчивых многообразий . . . . . . . . . . . . . . . . . . . . . . . . . 108

6.3. Аппроксимация дробных разрешающих семейств . . . . . . . . . . . . . . . . . . . . . . 111

Список литературы . . . . . . . . . . . . . . . . . . . . . . . . . . . . . . . . . . . . . . . . . . 119 


\section{ВВЕДЕНИЕ}

В данном обзоре представлены основы теории и наиболее интересные разработки задач для разрешающих семейств абстрактных полулинейных задач Коши в банаховом пространстве $E$

$$
\frac{d u(t)}{d t}=A u(t)+f(t, u(t)), \quad 0 \leqslant t<T, \quad u(0)=u^{0},
$$

где $A$ - оператор, порождающий экспоненциально затухающую компактную аналитическую $C_{0^{-}}$ полугруппу в банаховом пространстве $E$ и $f(\cdot, \cdot)$ - глобально липшицево и ограниченное отображение из $[0, T] \times E^{\theta}$ в $E$ (здесь $E^{\theta}$ - область определения $D\left((-A)^{\theta}\right)$ оператора $(-A)^{\theta}$, снабженная нормой графика). Интерес к таким задачам появился сравнительно недавно (см. $[22,88,91,116$, $132,136,213,218,236,242,265])$.

Для динамической системы

$$
u^{\prime}(t)=A u(t)+f(u(t)), \quad u(0)=u^{0},
$$

будет показано, что если абстрактная параболическая задача обладает лишь гиперболическими точками равновесия, то все точки равновесия изолированы и их имеется лишь конечное число, причем это число нечетно. Основные предположения, которые при этом налагаются, это компактность резольвент, гиперболичность любой точки равновесия и равномерная ограниченность нелинейной функции $f(\cdot)$. Кроме того, в случае, когда система имеет градиентную структуру, если предположить, что каждое глобальное решение сходится при $t \rightarrow \pm \infty$, то она имеет глобальный аттрактор, являющийся объединением неустойчивых многообразий точек равновесия. Мы допускаем довольно общие схемы дискретизации, следуя теории дискретных приближений, разработанной $\Phi$. Штуммелем, Р. Д. Григорьевым и Г. Вайникко. При всех этих предположениях мы рассматриваем аппроксимации аттракторов на общей аппроксимационной схеме, которая включает конечно-элементные, проекционные и конечно-разностные методы; затем мы докажем, что на этой аппроксимационной схеме аттракторы также являются полунепрерывными снизу.

Вообще говоря, доказано, что свойство равномерного притяжения глобальных аттракторов $\mathcal{A}_{n}$ динамических систем $S_{n}(\cdot)$ в банаховых пространствах $\left(E_{n},\|\cdot\|\right)$ при $n \rightarrow \infty$ эквивалентно непрерывной сходимости $\mathcal{A}_{n}$, определенной в терминах концепции дискретной сходимости. Результаты применяются к общим аппроксимационным схемам для абстрактных полулинейных параболических задач вида (**), где $A$ порождает экспоненциально убывающую компактную аналитическую $C_{0}$-полугруппу в банаховом пространстве $E$, а функция $f(\cdot): E^{\theta} \rightarrow E$ глобально липшицева и ограничена.

В статье разработан общий подход к установлению дискретной дихотомии в весьма общей формулировке и доказаны теоремы затенения, позволяющие сравнивать решения исходной задачи с решениями дискретных аппроксимаций по пространству и времени. В [79] в предположении, что резольвента компактна, была построена дискретизация по пространству. Хорошо известно (см. $[76,165,167,168])$, что фазовое пространство в окрестности гиперболической точки равновесия можно разложить таким образом, что исходная начальная задача сводится к начальным задачам с решениями, экспоненциально ограниченными на соответствующих подпространствах. Показано, что такое разложение потока сохраняется при достаточно общих аппроксимационных схемах, основанных на свойстве равномерного уплотнения. Основные предположения, при которых получены эти результаты, естественным образом выполнены, в частности, для операторов с компактными резольвентами и уплотняющими полугруппами (см. [50, 63]). 


\section{ПРЕДВАРИТЕЛЬНЫЕ СВЕДЕНИЯ}

Поведение гладких динамических систем в окрестности точки равновесия в значительной степени определяется поведением линеаризованной системы в этой окрестности, поэтому понятие устойчивости решения крайне важно для задач Коши $u^{\prime}(t)=A u(t), u(0)=u^{0}$, где $A$-линейный оператор в банаховом пространстве $E$.

Определение 1. $C_{0}$-Полугруппа $\exp (t A), t \geqslant 0$, на банаховом пространстве $E$ называется

(а) равномерно экспоненииально устойчивой, если существует такое $\mu>0$, что

$$
\lim _{t \rightarrow \infty} e^{\mu t}\|\exp (t A)\|=0
$$

(b) равномерно устойчивой, если

$$
\lim _{t \rightarrow \infty}\|\exp (t A)\|=0
$$

(с) сильно устойчивой, если

$$
\lim _{t \rightarrow \infty}\|\exp (t A) x\|=0 \quad \text { для любого } x \in E ;
$$

(d) слабо устойчивой, если

$$
\lim _{t \rightarrow \infty}\left\langle\exp (t A) x, x^{*}\right\rangle=0 \quad \text { для любых } x \in E, x^{*} \in E^{*} .
$$

Пожалуй, наиболее важные факты в этом направлении получены в [117].

Теорема 1. $C_{0}$-Полугруппа $\exp (t A), t \geqslant 0$, на гильбертовом пространстве $H$ является равномерно экспоненииально устойчивой тогда и только тогда, когда полуплоскость $\operatorname{Re} \lambda>0$ лежит в резольвентном множестве $\rho(A)$ u

$$
C=\sup _{\operatorname{Re} \lambda>0}\left\|(\lambda I-A)^{-1}\right\|<\infty .
$$

Подробное объяснение свойств, введенных в определении 1, приведено в [106]. Отметим следующий результат (см. [50,268]).

Теорема 2. Предположим, что равномерно ограниченная $C_{0}$-полугруппа $\exp (t A), t \geqslant 0$, на банаховом пространстве Е такова, что выполнены следующие условия:

(i) $\operatorname{P\sigma }\left(A^{*}\right) \cap i \mathbb{R}=\varnothing$;

(ii) множество $\sigma(A) \cap i \mathbb{R}$ счетно.

Тогда $C_{0}$-полугруппа $\exp (t A), t \geqslant 0$, силъно устойчива, т.е.

$$
\lim _{t \rightarrow \infty} \exp (t A) x=0 \quad \text { для всех } x \in E .
$$

Сильная устойчивость $C_{0}$-полугрупп изучалась многими авторами (см., например, $[7,73,215$, 249]). Отметим следующим результат.

Теорема 3. Предположим, что $C_{0}$-полугруппа является медленно меняющейся на бесконечности. Предел $\exp (t A) x_{0}$ при $t \rightarrow \infty$ существует тогда и только тогда, когда существует предел

в этом случае

$$
\lim _{0<\epsilon \rightarrow 0} \epsilon(\epsilon I-A)^{-1} x_{0}
$$

$$
\lim _{t \rightarrow \infty} \exp (t A) x_{0}=\lim _{0<\epsilon \rightarrow 0} \epsilon(\epsilon I-A)^{-1} x_{0}=y_{0} .
$$

Кроме того, элемент у принадлежит нуль-пространству $\mathcal{N}(A)$. Полугруппа $\exp (\cdot A)$ сильно устойчива (т.е. имеет место соотношение (***)) тогда и только тогда, когда

$$
\lim _{0<\epsilon \rightarrow 0} \epsilon(\epsilon I-A)^{-1} x=0 \quad \text { для любого } x \in E .
$$


Отметим следующий интересный факт: разрешающие семейства $S_{\alpha}(t, A), t \geqslant 0$, дробных уравнений порядка $0<\alpha<1$ никогда не убывают экспоненциально. Этот факт весьма важен для изучения сильно устойчивых разрешающих семейств.

Известно (см. [227]), что $\alpha$-разрешающие семейства являются частными случаями семейств резольвент, возникающих в теории интегральных уравнений Вольтерра

$$
S_{\alpha}(t) x=x+\int_{0}^{t} a(t-s) A S_{\alpha}(s) x d s,
$$

где $a(t)=t^{\alpha-1} / \Gamma(\alpha)$. Отметим следующий факт для аналитического $\alpha$-разрешающего семейства $S_{\alpha}(t)$ : если существует такое $\delta<0$, что $1 / \hat{a}(z) \in \rho(A)$ и преобразование Лапласа функции $S_{\alpha}(t)$ таково, что функция $\frac{(1 / \hat{a}(z)-A)^{-1}}{z \hat{a}(z)}$ аналитична при $\operatorname{Re} z>\delta$, то $S_{\alpha}(t)$ может быть экспоненциально устойчивой (см. [44]), но для функции $a(\cdot)$, имеющей вид функции экспоненциального типа (см. также [246]).

\section{ГЛАВА 1}

\section{ПОЛУЛИНЕЙНЫЕ УРАВНЕНИЯ}

Анализ полудискретизаций и дискретизаций по времени задач Коши при условии компактности резольвент для уравнений первого и второго порядка был проведен в $[25,26,108]$. В этой главе представлен анализ полудискретизаций периодических задач и задач Коши для уравнений первого порядка в банаховых пространствах.

\section{1. ДИФФЕРЕНЦИАЛЬНЫЕ УРАВНЕНИЯ В БАНАХОВЫХ ПРОСТРАНСТВАХ}

Пусть $B(E)$ - банахова алгебра всех линейных ограниченных операторов на комплексном банаховом пространстве $E$. Обозначим через $\mathcal{C}(E)$ множество всех линейных замкнутых плотно определенных операторов на $E$. Для оператора $B$ обозначим через $\sigma(B)$ его спектр, через $\rho(B)$ - резольвентное множество, через $\mathcal{N}(B)$ - нуль-пространство и через $\mathcal{R}(B)$ - множество значений. Напомним, что оператор $B \in B(E)$ называют фредгольмовым оператором, если $\mathcal{R}(B)$ замкнуто, $\operatorname{dim} \mathcal{N}(B)<\infty$ и $\operatorname{codim} \mathcal{R}(B)<\infty$. Индекс оператора $B$ - это число $\operatorname{ind}(B)=\operatorname{dim} \mathcal{N}(B)-\operatorname{codim} \mathcal{R}(B)$.

Рассмотрим в банаховом пространстве $E$ полулинейную задачу Коши

$$
v^{\prime}(t)=A v(t)+f(t, v(t)), \quad v(0)=v^{0}, \quad t \in \overline{\mathbb{R}}^{+}=[0, \infty),
$$

и $T$-периодическую задачу

$$
u^{\prime}(t)=A u(t)+f(t, u(t)), \quad u(t)=u(T+t), \quad t \in \overline{\mathbb{R}}^{+},
$$

где оператор $A$ порождает аналитическую компактную $C_{0}$-полугруппу, а достаточно гладкая функция $f(\cdot, \cdot)$ удовлетворяет условию $f(t, x)=f(t+T, x)$ для любых $x \in E$ и $t \in \overline{\mathbb{R}}^{+}$. Мы понимаем решения в классическом смысле (см. [262]). Существование решений для таких задач доказано, например, в $[9,21,62,68,91,132,136,137,206,207]$. В дальнейшем $A: D(A) \subseteq E \rightarrow E-$ замкнутый линейный оператор, удовлетворяющий условию

$$
\left\|(\lambda I-A)^{-1}\right\|_{B(E)} \leqslant \frac{M}{1+|\lambda|} \text { для люб' } \operatorname{Re} \lambda \geqslant 0 .
$$

При выполнении условия (1.1.3) спектр оператора $A$ лежит в девой полуплоскости: $\sup \{\operatorname{Re} \lambda: \lambda \in$ $\sigma(A)\}<0$; поэтому можно определить оператор дробной степени $(-A)^{\theta}, \theta \in \overline{\mathbb{R}}^{+}$, ассоциированный с $A$ и $E^{\theta}$ (см. $\left.[23,131,136,199]\right)$, где пространство $E^{\theta}:=D\left((-A)^{\theta}\right)$ снабжено нормой графика $\|x\|_{E^{\theta}}=\left\|(-A)^{\theta} x\right\|_{E}$. 
Обозначим через $v\left(\cdot ; v^{0}\right)$ решение задачи Коши (1.1.1) с начальными данными $v\left(0 ; v^{0}\right)=v^{0}$. Функция $v\left(\cdot ; v^{0}\right)$ удовлетворяет интегральному уравнению

$$
v(t)=\exp (t A) v^{0}+\int_{0}^{t} \exp ((t-s) A) f(s, v(s)) d s, \quad t \in[0, T] .
$$

Введем оператор сдвига $\mathcal{K}\left(v^{0}\right)=v\left(T ; v^{0}\right)$, отображающий $E$ в $E$. Если $v\left(\cdot ; x^{*}\right)$ - периодическое решение задачи (1.1.1), то $x^{*}$ - нуль компактного векторного поля $I-\mathcal{K}$, т.е. $\mathcal{K}\left(x^{*}\right)=x^{*}$.

Пусть $\Omega$-открытое множество в банаховом пространстве $F$ и $\mathcal{B}: \bar{\Omega} \rightarrow F$-компактный оператор, не имеющий неподвижных точек на границе множества $\Omega$. Для векторного поля $\mathcal{V}(x)=x-\mathcal{B} x$ введем целочисленную характеристику, так называемое вращение $\gamma(I-\mathcal{B} ; \partial \Omega)$ (см. [21]). Пусть $z^{*}$ - единственная изолированная неподвижная точка оператора $\mathcal{B}$ в шаре $\mathcal{U}_{F}\left(z^{*}, r_{0}\right)$ радиуса $r_{0}$ с центром в $z^{*}$. Тогда

$$
\gamma\left(I-\mathcal{B} ; \partial \mathcal{U}_{F}\left(z^{*}, r_{0}\right)\right)=\gamma\left(I-\mathcal{B} ; \partial \mathcal{U}_{F}\left(z^{*}, r\right)\right)
$$

для $0<r \leqslant r_{0}$; это общее значение вращений назовем индексом неподвижной точки $z^{*}$ и обозначим через $\operatorname{ind}\left(z^{*} ; I-\mathcal{B}\right)$.

Отметим, что результаты, касающиеся задач, подобных $(1.1 .1)$, в случае, когда $\exp (\cdot A)$ не компактная, но уплотняющая полугруппа, можно найти, например, в $[50,86,206,207]$.

Введем также такой оператор $K$, что решение задачи (1.1.2) удовлетворяет интегральному уравнению

$$
\begin{aligned}
& u(t)=(K u)(t) \equiv \\
& \equiv \exp (t A)(I-\exp (T A))^{-1} \int_{0}^{T} \exp ((T-s) A) f(s, u(s)) d s+\int_{0}^{t} \exp ((t-s) A) f(s, u(s)) d s,
\end{aligned}
$$

где $T$-период решения $u(\cdot)$. Оператор $K$ отображает пространство непрерывных функций $C([0, T] ; E)$ в себя.

Замечание 1.1.1. Предполагается, что в (1.1.5) оператор $(I-\exp (T A))^{-1}$ существует и ограничен. Однако достаточно предположить, что включение $(I-\exp (t A))^{-1} \in B(E)$ имеет место при $t \geqslant t_{0}$, где $t_{0}>0$. Это предположение не является весьма строгим, поскольку без ограничения общности можно заменить $A$ на $A-\omega I$ и получить

$$
\|\exp (t(A-\omega))\| \leqslant M e^{-\delta t}, \quad \delta>0, \quad t \geqslant 0 .
$$

Согласно [70] имеем

$$
(I-\exp (t A))^{-1} \in B(E), \quad t>0 .
$$

Замечание 1.1.2. В дальнейшем фразу «функиия $f(\cdot, \cdot)$ является достаточно гладкой» будем понимать в следующем смысле: функция $f(\cdot, \cdot)$ по крайней мере непрерывна по обоим аргументам, $\sup \|f(t, x)\| \leqslant C_{2}$ и такова, что существует глобальное обобщенное решение задачи (1.1.1) $\cdot \begin{aligned} & t \in[0, T], \\ & \|x\| \leqslant c_{1}\end{aligned}$

\section{2. НЕКОТОРЫЕ ИНТЕРЕСНЫЕ СВОЙСТВА}

Теорема 1.2.1 (принцип родственности; см. [80]). Предположим, что функция $f(\cdot, \cdot)$ является достаточно гладкой, так что существует изолированное обобщенное решение $u^{*}(\cdot)$ периодической задачи (1.1.2), элемент $x^{*}$ - неподвижная точка оператора $\mathcal{K}$ и резольвента $(\lambda I-A)^{-1}$ компактна. Тогда имеет место следующее равенство:

$$
\operatorname{ind}\left(x^{*} ; I-\mathcal{K}\right)=\operatorname{ind}\left(u^{*}(\cdot) ; I-K\right) .
$$

Доказательство. Пусть $S \equiv \mathcal{U}_{E}\left(x^{*}, \rho\right)$. Тогда вращение $\gamma(I-\mathcal{K} ; \partial S)$ поля $I-\mathcal{K}$ на сфере $\partial S$ равно индексу ind $\left(x^{*} ; I-\mathcal{K}\right)$ :

$$
\gamma(I-\mathcal{K} ; \partial S)=\operatorname{ind}\left(x^{*} ; I-\mathcal{K}\right) .
$$


Рассмотрим оператор $K$, определенный в (1.1.5), на пространстве

$$
F=C([0, T] ; E) \equiv\left\{u(t):\|u\|_{F}=\max _{t \in[0, T]}\|u(t)\|_{E}<\infty\right\} .
$$

Пусть

$$
M=\sup _{x \in S} \max _{0 \leqslant t \leqslant T}\|v(t ; x)\| .
$$

Рассмотрим область

$$
\Omega=\left\{u(\cdot) \in C([0, T] ; E): u(0) \in S,\|u(\cdot)\|_{F} \leqslant M+1\right\} \subset C([0, T] ; E) .
$$

Функция $u^{*}(\cdot)$ является единственным нулем компактного векторного поля $I-K$ на $\bar{\Omega}$; следовательно,

$$
\gamma(I-K ; \partial \Omega)=\operatorname{ind}\left(u^{*}(\cdot) ; I-K\right) .
$$

В силу (1.2.1) and (1.2.3), для завершения доказательства теоремы родственности достаточно показать, что

$$
\gamma(I-\mathcal{K} ; \partial S)=\gamma(I-K ; \partial \Omega)
$$

Для этого рассмотрим следующее семейство компактных векторных полей на $\partial \Omega$ :

$$
\begin{aligned}
\Phi(u(\cdot) ; \lambda)=u(t)-(1-\lambda) \exp (t A)(I-\exp (T A))^{-1} \int_{0}^{T} \exp ((T-s) A) f(s ; u(s)) d s- \\
-\lambda \exp (t A) \mathcal{K}(u(0))-\int_{0}^{t} \exp ((t-s) A) f(s, u(s)) d s, \quad 0 \leqslant \lambda \leqslant 1 .
\end{aligned}
$$

Поля $\Phi(u(\cdot) ; \lambda)$ невырождены на $\partial \Omega$. Действительно, если $\Phi\left(u_{0}(\cdot) ; \lambda_{0}\right)=0$ для некоторых $u_{0}(\cdot) \in \partial \Omega$ и $\lambda_{0} \in[0,1]$, то

$$
u_{0}(0)=\left(1-\lambda_{0}\right)(I-\exp (T A))^{-1} \int_{0}^{T} \exp ((T-s) A) f\left(s, u_{0}(s)\right) d s+\lambda_{0} u_{0}(T) .
$$

Поскольку из уравнения $(1.2 .6)$ и соотношения $\Phi\left(u_{0}(\cdot) ; \lambda_{0}\right)=0$ следует, что функция $u_{0}(\cdot)-$ обобщенное решение задачи (1.1.1), получаем

$$
\int_{0}^{T} \exp ((T-s) A) f\left(s, u_{0}(s)\right) d s=u_{0}(T)-\exp (T A) u_{0}(0) .
$$

Не умаляя общности, предположим, что $\operatorname{Re} \sigma(A)<0$. Из (1.2.6) и $(1.2 .7)$ получим

$$
\exp (T A)\left(\left(u_{0}(0)-u_{0}(T)\right)=\lambda_{0}^{-1}\left(u_{0}(0)-u_{0}(T)\right) .\right.
$$

Если $u_{0}(0)-u_{0}(T) \neq 0$, то этот элемент является собственным вектором оператора $\exp (T A)$, соответствующим собственному значению $\lambda_{0}^{-1}>1$. Однако это невозможно, поскольку $\operatorname{Re} \sigma(A)<0$ и $\sigma(\exp (T A)) \backslash\{0\}=e^{T \sigma(A)}$. Следовательно, $u_{0}(0)=u_{0}(T)$, так что $u_{0}(\cdot)$ является $T$-периодическим решением задачи (1.1.2) и нулем поля $I-K$; противоречие.

Поля семейства (1.2.5) невырождены на $\partial \Omega ;$ следовательно, поля $\Phi(u(\cdot) ; 0)=I-K$ и $\Phi(u(\cdot) ; 1)$ гомотопны на $\partial \Omega$. Получаем

$$
\gamma(I-K ; \partial \Omega)=\gamma(\Phi(u(\cdot) ; 1) ; \partial \Omega)
$$

Рассмотрим на $\partial \Omega$ следующее семейство векторных полей:

$$
\Psi(u(\cdot) ; \lambda)=u(t)-P_{\lambda}\left(\exp (t A) \mathcal{K}(u(0))+\int_{0}^{t} \exp ((t-s) A) f(s, u(s)) d s\right), \quad 0 \leqslant \lambda \leqslant T,
$$


где оператор $P_{\lambda}: F \rightarrow F$ определен следующим образом:

$$
\left(P_{\lambda} w\right)(t)= \begin{cases}w(t), & 0 \leqslant t \leqslant \lambda \\ w(\lambda), & \lambda \leqslant t \leqslant T\end{cases}
$$

Оператор

$$
Q(u(\cdot))(t)=\exp (t A) \mathcal{K}(u(0))+\int_{0}^{t} \exp ((t-s) A) f(s, u(s)) d s,
$$

отображающий $F$ to $F$, компактен. Оператор $P_{\lambda}: F \rightarrow F$ сильно непрерывен по $\lambda$. Следовательно, оператор $P_{\lambda} Q$ равномерно непрерывен по $\lambda$ и, таким образом, семейство (1.2.9) является компактной деформацией (см. [21, §19.1]).

Покажем, что семейство (1.2.9) невырождено на $\partial \Omega$. Предположим, что имеем

$$
u_{0}(\cdot) \neq v\left(\cdot ; x^{*}\right), \quad \Psi\left(u_{0}(\cdot) ; \lambda_{0}\right)=0
$$

для некоторых $\lambda_{0} \in[0,1]$ и $u_{0}(\cdot) \in \partial \Omega$. Граница $\partial \Omega$ области $\Omega$ состоит из двух множеств:

$$
\begin{aligned}
& G_{0}=\left\{u(\cdot) \in C([0, T] ; E): u(0) \in S,\|u(\cdot)\|_{C([0, T] ; E)}=M+1\right\}, \\
& G_{1}=\left\{u(\cdot) \in C([0, T] ; E): u(0) \in \partial S,\|u(\cdot)\|_{C([0, T] ; E)} \leqslant M+1\right\} .
\end{aligned}
$$

Пусть $u_{0}(\cdot) \in G_{0}$. Тогда

$$
\left\|u_{0}(\cdot)\right\|_{C([0, T] ; E)}=M+1 .
$$

С другой стороны, поскольку функция $u_{0}(\cdot)$ является решением задачи (1.1.1) на отрезке $[0, \lambda]$ и $u_{0}(0) \in S$, из (1.2.2) заключаем, что

$$
\left\|u_{0}(\cdot)\right\|_{C([0, T] ; E)}=\max _{0 \leqslant t \leqslant T}\left\|u_{0}(t)\right\|_{E}=\max _{0 \leqslant t \leqslant \lambda}\left\|u_{0}(t)\right\|_{E} \leqslant M .
$$

Равенства (1.2.10) и (1.2.11) несовместны. Таким образом, имеем единственную возможность: $u_{0}(\cdot) \in G_{1}$ и $u_{0}(0) \in \partial S$. Из соотношения $\Psi\left(u_{0}(\cdot) ; \lambda_{0}\right)=0$ следует, что $u_{0}(0)=\mathcal{K}\left(u_{0}(0)\right)$, что невозможно в силу выбора радиуса $\rho$ шара $S$. Следовательно, поля семейства (1.2.9) невырождены на $\partial \Omega$ и гомотопны друг другу. Итак,

$$
\gamma(\Psi(u(\cdot) ; 0) ; \partial \Omega)=\gamma(\Psi(u(\cdot) ; T) ; \partial \Omega) .
$$

Однако $\Psi(u(\cdot) ; T)=\Phi(u(\cdot) ; 1) ;$ следовательно,

$$
\gamma(\Psi(u(\cdot) ; 0) ; \partial \Omega)=\gamma(\Phi(u(\cdot) ; 1) ; \partial \Omega) .
$$

Рассмотрим поле

$$
\Psi(u(\cdot) ; 0)=u(t)-\mathcal{K}(u(0)), \quad u(\cdot) \in \partial \Omega .
$$

Поскольку оператор $\mathcal{K}(u(0))$ можно рассматривать как отображение из $F$ в пространство $\widetilde{E}$ постоянных функций, заключаем (см. [21]), что его вращение совпадает с вращением его ограничения $\widetilde{\Psi}$ на $\partial \Omega \cap \widetilde{E}$ и с вращением его ограничения $\widetilde{\Psi}(u(\cdot) ; 0)$ на $\partial \Omega \cap \widetilde{E}$. Поскольку поле $\widetilde{\Psi}(u(\cdot) ; 0)$ на $\partial \Omega \cap \widetilde{E}$ изоморфно $I-\mathcal{K}$ на $\partial S$, имеем

$$
\gamma(\Psi(u(\cdot) ; 0) ; \partial \Omega)=\gamma(\widetilde{\Psi}(u(\cdot) ; 0) ; \partial \Omega \cap \widetilde{E})=\gamma(I-\mathcal{K} ; \partial S) .
$$

Из уравнений (1.2.8), (1.2.12) и (1.2.13) получим (1.2.4). Теорема доказана.

Замечание 1.2.1. Для доказательства компактности оператора

$$
\int_{0}^{t} \exp ((t-s) A) f(s, u(s)) d s
$$


обычно используют полугрупповое свойство. В случае других сильно непрерывных компактных функций вместо $\exp (\cdot A)$ (например, синус оператор-функций) можно применить подход, предложенный в [264]. В случае дробных дифференциальных уравнений $D^{\alpha} u(t)=A u(t)+f(u(t))$ можно установить компактность оператора

$$
u(t)=S_{\alpha}(t) u^{0}+\int_{0}^{t} P_{\alpha}(t-s) f(u(s)) d s
$$

используя подход из [109].

Рассмотрим в пространстве $E$ следующую полулинейную неавтономную параболическую задачу:

$$
\begin{aligned}
& u^{\prime}(t)=A u(t)+f(t, u(t)), \quad t \in \overline{\mathbb{R}}^{+}, \\
& u(0)=u^{0} \in D(A),
\end{aligned}
$$

где $A$-оператор, порождающий аналитическую $C_{0}$-полугруппу, и $f(\cdot, \cdot)$ - достаточно гладкая функция. Классическое решение задачи (1.2.14) удовлетворяет уравнению

$$
u(t)=\tilde{K} u(t) \equiv \exp (t A)+\int_{0}^{t} \exp ((t-s) A) f(s, u(s)) d s, \quad t \in \overline{\mathbb{R}}^{+},
$$

где $\tilde{K}$ рассматривается как оператор из $F$ в $F$. Нам понадобится следующий результат.

Теорема 1.2 .2 (см. [128]). Пусть $A-$ генератор аналитической $C_{0}$-полугруппь, резольвента $(\lambda I-A)^{-1}$ компактна для некоторого $\lambda \in \rho(A)$ и оператор $\tilde{K}$ определен формулой (1.2.15). Если $u^{*}(\cdot)$ - единственное обобщенное решение задачи $(1.2 .14), \operatorname{mo} \operatorname{ind}\left(u^{*}(\cdot) ; I-\tilde{K}\right)=1$.

Если оператор $A$ такой, как описано выше, и число $0<\theta<1$ фиксировано, рассмотрим следующую полулинейную автономную параболическую задачу в пространстве $E^{\theta}$ :

$$
\begin{aligned}
& u^{\prime}(t)=A u(t)+f(u(t)), \quad t \in \overline{\mathbb{R}}^{+}, \\
& u(0)=u^{0} \in E^{\theta}
\end{aligned}
$$

где $f(\cdot): E^{\theta} \subseteq E \rightarrow E$ - глобально липшицева, ограниченная и непрерывно дифференцируемая по Фреше функция.

Отметим, что даже в случае, когда

$$
u(t)=\exp (t A) u^{0}+\int_{0}^{t} \exp ((t-s) A) g(s) d s, \quad t \geqslant 0,
$$

и $C_{0}$-полугруппа $\exp (\cdot A)$ аналитична, функция $u(\cdot)$ не обязательно дифференцируема, если $g(\cdot) \in$ $C([0, T] ; E)$, т.е. задача

$$
\begin{aligned}
& u^{\prime}(t)=A u(t)+g(t), \quad t \in[0, T], \\
& u(0)=u_{0},
\end{aligned}
$$

не является корректно поставленной в классическом смысле в пространстве $C([0, T] ; E)$ для общего банахова пространства $E$. Однако задача (1.2.17) корректно поставлена в классическом смысле в $C\left([0, T] ; E^{\theta}\right)$ (см. $\left.[30,98,99]\right)$, где $E^{\theta}=(E, D(A))_{\theta}$ - подходящее интерполяционное пространство. Кроме того, если иметь в виду приложения к пространствам $E=L^{p}(\Omega)$ потребовать дифференцируемость по Фреше функции $f(\cdot): L^{2}(\Omega) \rightarrow L^{2}(\Omega)$, то функция $f(\cdot)$ должна быть линейной (см. [42]). Чтобы включить в рассмотрение более общие нелинейности, требуется более слабое предположение, что функция $f(\cdot): H_{0}^{1}(\Omega) \rightarrow L^{2}(\Omega)$ дифференцируема по Фреше. Трудности, вызываемые этими фактами, могут быть разрешены рассмотрением задачи (1.2.16) в банаховом пространстве $E^{\theta}, 0 \leqslant \theta<1$, в предположении, что $f(\cdot): E^{\theta} \rightarrow E$ дифференцируема по Фреше, причем производная в точке равновесия $f^{\prime}\left(u^{*}\right) \in B\left(E^{\theta}, E\right)$. В случае $E=L^{2}(\Omega)$ и 
$A=\Delta$, мы, как обычно, имеем $E^{1 / 2}=H_{0}^{1}(\Omega)$ при $\theta=1 / 2$. Теорему 1.2 .2 можно доказать и для задачи (1.2.16).

\subsection{TeOpema ABC}

Ф. Штуммель, Р. Д. Григорьев и Г. Вайникко предложили идею дискретной сходимости, или $\mathcal{P}$-сходимости в контексте, названном ими общей аппроксимационной схемой (см. [119-123, 244, $256,259])$, которую можно описать следующим образом. Пусть $E_{n}$ и $E$-банаховы пространства и $\left\{p_{n}\right\}$ - последовательность линейных ограниченных операторов $p_{n}: E \rightarrow E_{n}, p_{n} \in B\left(E, E_{n}\right)$, $n \in \mathbb{N}=\{1,2, \ldots\}$, удовлетворяющих следующему условию согласованности норм:

$$
\left\|p_{n} x\right\|_{E_{n}} \rightarrow\|x\|_{E} \quad \text { при } n \rightarrow \infty \text { для любого } x \in E .
$$

Из принципа равномерной ограниченности (см. [274]) получаем следующее утверждение.

Лемма 1.3.1 (см. [259]). Найдется такая константа $C \geqslant 1$, что $\left\|p_{n}\right\|_{B\left(E, E_{n}\right)} \leqslant C$ для всех $n \in \mathbb{N}$.

Определение 1.3.1. Будем говорить, что последовательность $\left\{x_{n}\right\}, x_{n} \in E_{n}, n \in \mathbb{N}, \mathcal{P}$-сходится $\kappa x \in E$ (обозначение $x_{n} \stackrel{\mathcal{P}}{\longrightarrow} x$ ), если $\left\|x_{n}-p_{n} x\right\|_{E_{n}} \rightarrow 0$ при $n \rightarrow \infty$.

Определение 1.3.2. Будем говорить, что последовательность ограниченных линейных операторов $B_{n} \in B\left(E_{n}\right), n \in \mathbb{N}, \mathcal{P} \mathcal{P}$-сходится к ограниченному линейному оператору $B \in B(E)$ (обозначение $B_{n} \stackrel{\mathcal{P} \mathcal{P}}{\longrightarrow} B$ ), если $B_{n} x_{n} \stackrel{\mathcal{P}}{\longrightarrow} B x$ для любого $x \in E$ и любой последовательности $\left\{x_{n}\right\}, x_{n} \in E_{n}, n \in \mathbb{N}$, удовлетворяющей условию $x_{n} \stackrel{\mathcal{P}}{\longrightarrow} x$.

Рассмотрим следующую корректно поставленную задач Коши в банаховом пространстве $E$ с оператором $A \in \mathcal{C}(E)$ :

$$
u^{\prime}(t)=A u(t), \quad t \in \in \overline{\mathbb{R}}^{+}, \quad u(0)=u^{0}
$$

оператор $A$ порождает $C_{0}$-полугруппу $\exp (\cdot A)$. Хорошо известно (см. [11]), что решение задачи (1.3.2) можно записать при помощи этой $C_{0}$-полугруппы: $u(t)=\exp (t A) u^{0}, t \geqslant 0$.

В рамках общей дискретизационной схемы рассмотрим следующую полудискретную аппроксимацию задачи (1.3.2) в банаховых пространствах $E_{n}$ :

$$
u_{n}^{\prime}(t)=A_{n} u_{n}(t), \quad t \in \in \overline{\mathbb{R}}^{+}, \quad u_{n}(0)=u_{n}^{0},
$$

где операторы $A_{n} \in \mathcal{C}\left(E_{n}\right)$ порождают $C_{0}$-полугруппы, согласованные с оператором $A$, и $u_{n}^{0} \stackrel{\mathcal{P}}{\longrightarrow} u^{0}$.

Приведем следующую версию теоремы Троттера-Като (только для аналитических $C_{0}$-полугрупп), которая дает решение задачи о сходимости решений задач (1.3.3) к решению задачи $(1.3 .2)$.

Теорема 1.3.1 (см. $[26,127,219]$, теорема АВС). Предположим, что операторы $A$ и $A_{n}$ порождают аналитические $C_{0}$-полугруппь. Следующие условия $(\mathrm{A})$ и $\left(\mathrm{B}_{1}\right)$ эквивалентны условию $\left(\mathrm{C}_{1}\right)$ :

(А) согласованность: существует такое $\lambda \in\left[\bigcap_{n} \rho\left(A_{n}\right)\right] \cap \rho(A)$, что резольвенты сходятся:

$$
\left(\lambda I_{n}-A_{n}\right)^{-1} \stackrel{\mathcal{P P}}{\longrightarrow}(\lambda I-A)^{-1}
$$

$\left(\mathrm{B}_{1}\right)$ устойчивость: существуют такие константы $M_{2} \geqslant 1 u \omega_{2}$, что

$$
\left\|\left(\lambda I_{n}-A_{n}\right)^{-1}\right\|_{B\left(E_{n}\right)} \leqslant \frac{M_{2}}{\left|\lambda-\omega_{2}\right|}, \quad \operatorname{Re} \lambda>\omega_{2}, \quad n \in \mathbb{N}
$$

$\left(\mathrm{C}_{1}\right)$ сходимость: для любого конечного $\mu>0$ и некоторого $0<\varphi<\pi / 2$ имеем

$$
\max _{\eta \in \Sigma(\varphi, \mu)}\left\|\exp \left(\eta A_{n}\right) u_{n}^{0}-p_{n} \exp (\eta A) u^{0}\right\|_{E_{n}} \rightarrow 0
$$

nрu $n \rightarrow \infty$, если $u_{n}^{0} \stackrel{\mathcal{P}}{\longrightarrow} u^{0}$. 
3деcъ

$$
\Sigma(\varphi, \mu)=\{z \in \Sigma(\varphi):|z| \leqslant \mu\}, \quad \Sigma(\varphi)=\{z \in \mathbb{C}:|\arg (z)| \leqslant \varphi\} .
$$

Итак, при исследовании полудискретизации полулинейной задачи (1.1.2) будем считать без ограничения общности, что условия $(\mathrm{A})$ и $\left(\mathrm{B}_{1}\right)$ выполнены.

\section{4. СХОДИМОСТЬ ПОЛУДИСКРЕТИЗАЦИЙ ДЛЯ ПЕРИОДИЧЕСКОЙ ЗАДАЧИ}

Под полудискретной аппроксимацией $T$-периодической задачи (1.1.2) будем понимать $T$-периодические задачи

$$
u_{n}^{\prime}(t)=A_{n} u_{n}(t)+f_{n}\left(t, u_{n}(t)\right), \quad u_{n}(t)=u_{n}(t+T), \quad t \in \overline{\mathbb{R}}^{+},
$$

где операторы $A_{n}$ порождают аналитические полугруппы в $E_{n}$, выполнено условие $(\mathrm{A})$, функции $f_{n}(\cdot, \cdot)$ равномерно ограничены:

$$
\sup _{\substack{t \in[0, T],\left\|x_{n}\right\| \leqslant c_{1}}}\left\|f_{n}\left(t, x_{n}\right)\right\| \leqslant C_{2},
$$

функции $f_{n}$ аппроксимируют $f$, являются достаточно гладкими и удовлетворяют условию $f_{n}\left(t, x_{n}\right)=f_{n}\left(t+T, x_{n}\right)$ для любых $x_{n} \in E_{n}$ и $t \in \overline{\mathbb{R}}^{+}$.

Обобщенные решения задач (1.4.1) определяются уравнениями

$$
\begin{aligned}
u_{n}(t)=\left(K_{n} u_{n}\right)(t) \equiv \exp \left(t A_{n}\right)\left(I_{n}-\exp \left(T A_{n}\right)\right)^{-1} & \int_{0}^{T} \exp \left((T-s) A_{n}\right) f_{n}\left(s, u_{n}(s)\right) d s+ \\
& +\int_{0}^{t} \exp \left((t-s) A_{n}\right) f_{n}\left(s, u_{n}(s)\right) d s .
\end{aligned}
$$

К этим задачам в литературе проявлен большой интерес (см., например, работы $[40,136,137]$ посвященные задачам (1.1.1)-(1.1.2)). Особенно интересна разработка численного анализа для таких задач, поскольку многие модели (например, различные физические модели, модели популяционной динамики и т. д., см. $[132,136])$ могут быть описаны абстрактными параболическими уравнениями. Условия, при которых исследовались полудискретизации, являются весьма общими. Введем следующие понятия.

Определение 1.4.1. Последовательность элементов $\left\{x_{n}\right\}, x_{n} \in E_{n}, n \in \mathbb{N}$, назовем $\mathcal{P}$-комnактной, если для любого $\mathbb{N}^{\prime} \subseteq \mathbb{N}$ существуют такие $\mathbb{N}^{\prime \prime} \subseteq \mathbb{N}^{\prime}$ и $x \in E$, что $x_{n} \stackrel{\mathcal{P}}{\longrightarrow} x$ при $n \rightarrow \infty$ в $\mathbb{N}^{\prime \prime}$.

Определение 1.4.2. Функция $\mu(\cdot)$ называется дискретной мерой некомпактности, если для любой ограниченной последовательности $\left\{x_{n}\right\}, x_{n} \in E_{n}$, имеем

$$
\mu\left(\left\{x_{n}\right\}\right)=\inf \left\{\epsilon>0: \forall \mathbb{N}^{\prime} \subseteq \mathbb{N} \exists \mathbb{N}^{\prime \prime} \subseteq \mathbb{N}^{\prime} \& x^{\prime} \in E \text { такие, что }\left\|x_{n}-p_{n} x^{\prime}\right\| \leqslant \epsilon, n \in \mathbb{N}^{\prime \prime}\right\} .
$$

Определение 1.4.3. Будем говорить, что последовательность операторов $\left\{\mathcal{B}_{n}\right\}, \mathcal{B}_{n}: E_{n} \rightarrow E_{n}$, сходится компактно к оператору $\mathcal{B}, \mathcal{B}: E \rightarrow E$, если $\mathcal{B}_{n} \stackrel{\mathcal{P} \mathcal{P}}{\longrightarrow} \mathcal{B}$ дискретно и выполнено следующее условие компактности:

$$
\text { из }\left\|x_{n}\right\|=O(1) \text { следует, что }\left\{\mathcal{B}_{n} x_{n}\right\} \text { является } \mathcal{P} \text {-компактной. }
$$

Определение 1.4.4. Решение $v(\cdot)$ задачи Коши (1.1.1) называется устойчивым в смысле Ляnунова, если для любого $\epsilon>0$ найдется такое $\delta>0$, что из неравенства

$$
\|v(0)-\tilde{v}(0)\| \leqslant \delta
$$

следует

$$
\max _{0 \leqslant t<\infty}\|v(t)-\tilde{v}(t)\| \leqslant \epsilon
$$

где $\tilde{v}(\cdot)$ - обобщенное решение задачи (1.1.1) с начальным значением $\tilde{v}(0)$. 
Определение 1.4.5. Решение $v(\cdot)$ задачи Коши (1.1.1) называется равномерно асимптотичеcки устойчивым в точке $v(0)$, если оно устойчиво в смысле Ляпунова и для любого обобщенного решения $\tilde{v}(\cdot)$ задачи (1.1.1), удовлетворяющего условию $\|v(0)-\tilde{v}(0)\| \leqslant \delta$, имеем

$$
\lim _{t \rightarrow \infty}\|v(t)-\tilde{v}(t)\|=0
$$

равномерно по $\tilde{v}(\cdot) \in B(v(0) ; \delta)$, т.е. существует такая функция $\phi_{v(0), \delta}(\cdot)$, что

$$
\|v(t ; v(0))-v(t ; \tilde{v}(0))\| \leqslant \phi_{v(0), \delta}(t),
$$

где $\phi_{v(0), \delta}(t) \rightarrow 0$ при $t \rightarrow \infty$ и $\|v(0)-\tilde{v}(0)\| \leqslant \delta$.

Замечание 1.4.1. Достаточные условия существования равномерно асимптотически устойчивого решения задачи (1.1.1) приведены, например, в [136, теорема 8.1.1]; они связаны с положением спектра оператора $A+\frac{\partial f}{\partial v}\left(t, v^{*}(t)\right)$.

Напомним следующую теорему.

Теорема 1.4.1 (см. [80]). Предположим, что условия (A) и (B 1 ) выполнены и компактные резольвенты $R(\lambda ; A)$ и $R\left(\lambda ; A_{n}\right)$ сходятся компактно для некоторого $\lambda \in \rho(A)$ :

$$
\left(\lambda I_{n}-A_{n}\right)^{-1} \stackrel{\mathcal{P P}}{\longrightarrow}(\lambda I-A)^{-1} .
$$

Предположим такюе, что выполнены следующие условия:

(i) функиии $f$ u $f_{n}$ являются достаточно гладкими, так что существует такое изолированное обобщенное решение $u^{*}(\cdot)$ периодической задачи (1.1.2) с начальным условием $u^{*}(0)=x^{*}$, что задача Коши

$$
v^{\prime}(t)=A v(t)+f(t, v(t)), \quad v(0)=x^{*},
$$

имеет равномерно асимптотически устойчивое изолированное решение в точке $x^{*}$;

(ii) $f_{n}\left(t, x_{n}\right) \stackrel{\mathcal{P}}{\longrightarrow} f(t, x)$ для любого $t \in[0, T]$ при $x_{n} \stackrel{\mathcal{P}}{\longrightarrow} x$.

Тогда почти для всех п задачи (1.4.1) имеют периодические обобщенные решения $u_{n}^{*}(t), t \in[0, T]$, в окрестности точки $p_{n} u^{*}(\cdot)$, где $u^{*}(\cdot)$ - обобщенное периодическое решение задачи (1.1.2) с начальным условием $u^{*}(0)=x^{*}$. Кажсдая последовательность $\left\{u_{n}^{*}(\cdot)\right\}$ является $\mathcal{P}$-компактной $u$ $u_{n}^{*}(t) \stackrel{\mathcal{P}}{\longrightarrow} u^{*}(t)$ равномерно по $t \in[0, T]$.

Доказательство. Разобьем доказательство на несколько шагов.

Шаг 1. Во-первых, покажем, что компактная сходимость резольвент $R\left(\lambda ; A_{n}\right) \stackrel{\mathcal{P P}}{\longrightarrow} R(\lambda ; A)$ эквивалентна компактной сходимости $C_{0}$-полугрупп $\exp \left(t A_{n}\right) \rightarrow \exp (t A)$ для любого $t>0$. Пусть $\left\|x_{n}\right\|=O(1)$. Тогда из оценки

$$
\left\|A_{n} \exp \left(t A_{n}\right)\right\| \leqslant \frac{M}{t} e^{\omega t}
$$

получаем ограниченность последовательности $\left\{\left(A_{n}-\lambda I_{n}\right) \exp \left(t A_{n}\right) x_{n}\right\}$. Благодаря компактной сходимости резольвент можно сделать заключение о компактности последовательности $\left\{\exp \left(t A_{n}\right) x_{n}\right\}$.

Необходимость будет доказана, если для меры некомпактности $\mu(\cdot)$ (см. определение в $[26,259$, 262]) мы установим, что

$$
\mu\left(\left\{\left(\lambda I_{n}-A_{n}\right)^{-1} x_{n}\right\}\right)=0 \text { для }\left\|x_{n}\right\|=O(1) .
$$


Имеем

$$
\begin{aligned}
& \mu\left(\left\{\left(\lambda-A_{n}\right)^{-1} x_{n}\right\}\right)=\mu\left(\left\{\int_{0}^{\infty} e^{-\lambda t} \exp \left(t A_{n}\right) x_{n}\right\}\right) \leqslant \mu\left(\left\{\int_{0}^{q} e^{-\lambda t} \exp \left(t A_{n}\right) x_{n} d t\right\}\right)+ \\
& +\mu\left(\left\{\int_{Q}^{\infty} e^{-\lambda t} \exp \left(t A_{n}\right) x_{n} d t\right\}\right)+\mu\left(\left\{\exp \left(\epsilon A_{n}\right) \int_{q}^{Q} e^{-\lambda t} \exp \left((t-\epsilon) A_{n}\right) x_{n} d t\right\}\right) .
\end{aligned}
$$

Первые два члена справа могут быть сделаны малыми (меньше, чем $\epsilon$ ) подходящим выбором $q$ и $Q$. Последний член равен нулю в силу компактной сходимости $\exp \left(\epsilon A_{n}\right) \stackrel{\mathcal{P} \mathcal{P}}{\longrightarrow} \exp (\epsilon A)$ для любого $0<\epsilon<q$.

Шаг 2. Оператор $K$, определенный формулой (1.1.5), компактен в пространстве $F$. Действительно, следуя [216, с. 192], видим, что оператор

$$
\mathcal{F}_{\epsilon}\left(u_{k}\right)(t)=\exp (\epsilon A) \int_{0}^{t-\epsilon} \exp ((t-s-\epsilon) A) f\left(s, u_{k}(s)\right) d s
$$

отображает любое ограниченное множество функций $\left\{u_{k}(\cdot)\right\},\left\|u_{k}(\cdot)\right\|_{F} \leqslant C$, в компактное множество в $E$ для любых $t>0$ и $0<\epsilon<t$. Кроме того,

$$
\left\|\mathcal{F}_{\epsilon}\left(u_{k}\right)(t)-\mathcal{F}\left(u_{k}\right)(t)\right\| \leqslant C \epsilon \quad \text { для любого } t \in(0, T]
$$

где

$$
\mathcal{F}\left(u_{k}\right)(t)=\int_{0}^{t} \exp ((t-s) A) f\left(s, u_{k}(s)\right) d s
$$

и $0<\epsilon<t$. Заключаем, что оператор $\mathcal{F}(\cdot)(t): F \rightarrow E$ компактен для тех же самых $t>0$. При $t=0$ оператор $\mathcal{F}(\cdot)(0)$ также компактен. Кроме того, множество функций $\left\{F_{k}(\cdot)\right\}, F_{k}(t)=\mathcal{F}\left(u_{k}\right)(t)$, $t \in[0, T]$, является эквиограниченным и равностепенно непрерывным семейством, поскольку для $0<t_{1}<t_{2}$ имеем

$$
\left\|F_{k}\left(t_{2}\right)-F_{k}\left(t_{1}\right)\right\| \leqslant C\left(\int_{0}^{t_{1}}\left\|\exp \left(\left(t_{2}-s\right) A\right)-\exp \left(\left(t_{1}-s\right) A\right)\right\| d s+\left|t_{2}-t_{1}\right|\right),
$$

и $\exp (\cdot A)$ равномерно непрерывна при $t>0$.

Последовательность $\left\{y_{k}\right\}$, где

$$
y_{k}=(I-\exp (T A))^{-1} \int_{0}^{T} \exp ((T-s) A) f\left(s, u_{k}(s)\right) d s \in E,
$$

компактна, поскольку $\left\{\mathcal{F}\left(u_{k}\right)(T)\right\}$ - компактное множество. Таким образом, $\left\{\exp (\cdot A) y_{k}\right\}-$ компактная последовательность функций в $F$. По обобщенной теореме Арцела-Асколи оператор $K$ компактен.

Шаг 3. Рассмотрим операторы $K_{n}$, определенные формулой (1.4.2) на пространствах

$$
F_{n}=C\left([0, T] ; E_{n}\right) \equiv\left\{u_{n}(t):\left\|u_{n}\right\|_{F_{n}}=\max _{t \in[0, T]}\left\|u_{n}(t)\right\|_{E_{n}}<\infty\right\} .
$$

Легко видеть, что $K_{n} \stackrel{\mathcal{P P}}{\longrightarrow} K$. Действительно,

$$
I_{n} \stackrel{\mathcal{P P}}{\longrightarrow} I \text { устойчиво, } \quad \exp \left(T A_{n}\right) \stackrel{\mathcal{P P}}{\longrightarrow} \exp (T A) \text { компактно; }
$$

следовательно,

$$
I_{n}-\exp \left(T A_{n}\right) \rightarrow I-\exp (T A) \text { собственно, }
$$


нуль-пространство $\mathcal{N}(I-\exp (T A))$ тривиально, т.е. $\mathcal{N}(I-\exp (T A))=\{0\}$, и $I_{n}-\exp \left(T A_{n}\right)-$ фредгольмов оператор нулевого индекса. Тогда из [259] следует, что

$$
I_{n}-\exp \left(T A_{n}\right) \stackrel{\mathcal{P P}}{\longrightarrow} I-\exp (T A) \text { устойчиво, }
$$

т.е.

$$
\left(I_{n}-\exp \left(T A_{n}\right)\right)^{-1} \stackrel{\mathcal{P P}}{\longrightarrow}(I-\exp (T A))^{-1},
$$

и сходимость $K_{n} \stackrel{\mathcal{P P}}{\longrightarrow} K$ вытекает из теоремы о мажорантной сходимости. Чтобы показать, что $K_{n} \rightarrow K$ компактно, предположим, что $\left\|u_{n}\right\|_{F_{n}}=O(1)$. Тогда $\left\{K_{n} u_{n}\right\}$ является $\mathcal{P}$-компактной по обобщенной теореме Арцела-Асколи (см. [22] или [216, с. 192-193]). Чтобы это доказать, проверим, что мера некомпактности обращается в нуль, $\mu\left(\left\{\left(K_{n} u_{n}\right)(t)\right\}\right)=0$, для всех $t \in[0, T]$. Рассмотрим равенство

$$
\left(K_{n} u_{n}\right)(t)=\exp \left(t A_{n}\right) y_{n}+\psi_{n}^{\tau}(t)+\varphi_{n}^{\tau}(t)
$$

где

$$
\begin{aligned}
& y_{n}=\left(I_{n}-\exp \left(T A_{n}\right)\right)^{-1} \int_{0}^{T} \exp \left((T-s) A_{n}\right) f_{n}\left(s, u_{n}(s)\right) d s \\
& \psi_{n}^{\tau}(t)=\exp \left(\tau A_{n}\right) \int_{0}^{t-\tau} \exp \left((t-s-\tau) A_{n}\right) f_{n}\left(s, u_{n}(s)\right) d s \\
& \varphi_{n}^{\tau}(t)=\int_{t-\tau}^{t} \exp \left((t-s) A_{n}\right) f_{n}\left(s, u_{n}(s)\right) d s .
\end{aligned}
$$

В силу ограниченности $\left\|f_{n}\left(\cdot, u_{n}(\cdot)\right)\right\|_{F_{n}}$ можно выбрать $\left\|\varphi_{n}^{\tau}(\cdot)\right\|_{F_{n}}$ достаточно малым при достаточно малом $\tau$ и $\mu\left(\left\{\psi_{n}^{\tau}\right\}\right)=0$. Последовательность $\left\{y_{n}\right\}$ является $\mathcal{P}$-компактной, что и завершает проверку.

Шаг 4. Из условия существования изолированного равномерно асимптотически устойчивого решения $v\left(t ; x^{*}\right)$ задачи (1.4.3) вытекает, что в малой окрестности точки $x^{*}$ (обозначим ее $\mathcal{U}\left(x^{*}, \rho\right) \subset E$ ) оператор $\mathcal{K}$ компактен, поскольку множество $\mathcal{F}\left(u_{k}\right)(T)$ компактно для любой $\left\{u_{k}\right\}, u_{k}(t) \in \mathcal{U}\left(x^{*}, \epsilon\right), t \in[0, T]$, при условии $\left\|u_{k}(0)-x^{*}\right\| \leqslant \delta$. Точка $x^{*}$ является изолированным нулем компактного векторного поля $I-\mathcal{K}$, и индекс ind $\left(x^{*} ; I-\mathcal{K}\right)$ определен. Аналогично, функция $u^{*}(t)=v\left(t ; x^{*}\right), t \in[0, T]$, являющаяся решением задачи (1.1.5), - изолированный нуль поля $I-K$, и $\operatorname{ind}\left(u^{*}(\cdot) ; I-K\right)$ определен.

Шаг 5. Из условия равномерной асимптотической устойчивости решения $v(\cdot)$ задачи (1.1.1) в точке $x^{*}$ следует, что найдется такое целое число $m$, что оператор $\mathcal{K}^{m}$ отображает шар $\mathcal{U}\left(x^{*}, \delta\right)$ в себя; точнее,

$$
\left\|\mathcal{K}^{m}\left(x^{*}\right)-\mathcal{K}^{m}(x)\right\| \leqslant \phi_{x^{*}, \delta}(m T)<\delta \quad \text { для любого } x \in \mathcal{U}\left(x^{*}, \delta\right) .
$$

Это означает, что $\operatorname{ind}\left(x^{*} ; I-\mathcal{K}^{m}\right)=1$, и по $[21$, теорема 31.1$]$ получим, что $\operatorname{ind}\left(x^{*} ; I-\mathcal{K}\right)=1$. Используя шаг 4 и принцип родственности, имеем $\operatorname{ind}\left(u^{*}(\cdot) ; I-K\right)=1$. Итак, $K_{n} \rightarrow K$ компактно и $\operatorname{ind}\left(u^{*}(\cdot) ; I-K\right)=1$. Применяя результат из [259], заключаем, что множество решений задач (1.4.2) непусто, любая последовательность решений $\left\{u_{n}^{*}(\cdot)\right\}$ является $\mathcal{P}$-компактной и, кроме того, $u_{n}^{*}(t) \stackrel{\mathcal{P P}}{\longrightarrow} u^{*}(t)$ равномерно по $t \in[0, T]$ при $n \rightarrow \infty$. Теорема доказана.

Замечание 1.4.2. Условие (i) теоремы 1.4 .1 можно заменить на одно из следующих двух условий:

$\left(\mathrm{i}^{\prime}\right)$ найдется такая изолированная неподвижная точка $x^{*}$ оператора $\mathcal{K}$, что выполняется неравенство ind $\left(x^{*}, I-\mathcal{K}\right) \neq 0$ или

$\left(\mathrm{i}^{\prime \prime}\right)$ найдется такая изолированная неподвижная точка $u^{*}(\cdot)$ оператора $K$, что выполняется неравенство $\operatorname{ind}\left(u^{*}(\cdot), I-K\right) \neq 0$. 
Напомним, что каждое обобщенное решение задачи (1.2.16) удовлетворяет уравнению

$$
u(t)=(\tilde{K} u)(t) \equiv \exp (t A) u^{0}+\int_{0}^{t} \exp ((t-s) A) f(u(s)) d s, \quad t \in[0, T], \quad T<\infty .
$$

Рассмотрим также в банаховых пространствах $E_{n}^{\theta}$ следующее семейство параболических задач:

$$
\begin{aligned}
& u_{n}^{\prime}(t)=A_{n} u_{n}(t)+f_{n}\left(u_{n}(t)\right), \quad t \in \overline{\mathbb{R}}^{+}, \\
& u_{n}(0)=u_{n}^{0} \in E_{n}^{\theta},
\end{aligned}
$$

где $u_{n}^{0} \stackrel{\mathcal{P}}{\longrightarrow} u^{0}$, операторы $A_{n}$ и $A$ согласованы и $f_{n}(\cdot): E_{n}^{\theta} \rightarrow E_{n}$ - ограниченные липшицнепрерывные функции. Также предположим, что $f_{n}\left(x_{n}\right) \stackrel{\mathcal{P}}{\longrightarrow} f(x)$ и $f_{n}^{\prime}\left(x_{n}\right) \stackrel{\mathcal{P}^{\theta} \mathcal{P}}{\longrightarrow} f^{\prime}(x)$ при $x_{n} \stackrel{\mathcal{P}^{\theta}}{\longrightarrow} x$.

Согласно теореме 1.3.1, предположим, что операторы $A_{n}$ порождают (см. $\left.\left(\mathrm{B}_{1}\right)\right)$ аналитические $C_{0}$-полугруппы, удовлетворяющие оценкам

$$
\left\|e^{t A_{n}}\right\| \leqslant M e^{-t \tilde{\omega}}, \quad t \geqslant 0
$$

где $\tilde{\omega}>0$, резольвенты $\left(\lambda I_{n}-A_{n}\right)^{-1}$ компактны при некотором $\lambda \in \rho\left(A_{n}\right)$ и сходятся компактно для некоторого $\lambda \in \bigcap_{n=1}^{\infty} \rho\left(A_{n}\right) \cap \rho(A)$ :

$$
\left(\lambda I_{n}-A_{n}\right)^{-1} \stackrel{\mathcal{P P}}{\longrightarrow}(\lambda I-A)^{-1},
$$

а функции $f_{n}(\cdot): E_{n}^{\theta} \rightarrow E_{n}$ глобально липшицевы и ограничены, т.е. найдется такая константа $\tilde{c}>0$, что

$$
\left\|f_{n}\left(w_{n}\right)\right\|_{E_{n}} \leqslant \tilde{c} \quad \text { для всех } w_{n} \in E_{n}^{\theta},
$$

и непрерывно дифференцируемы по Фреше.

Пусть $t \mapsto u_{n}\left(t, u_{n}^{0}\right)$ - решение задачи (1.4.5). Известно, что все решения задач (1.4.5) глобально определены; таким образом, $t \mapsto u_{n}(t, \cdot)$ - непрерывная нелинейная полугруппа для каждого $n \in \mathbb{N}$. Рассмотрим операторные уравнения

$$
u_{n}(t)=\left(\tilde{K}_{n} u_{n}\right)(t) \equiv \exp \left(t A_{n}\right) u_{n}^{0}+\int_{0}^{t} \exp \left((t-s) A_{n}\right) f_{n}\left(u_{n}(s)\right) d s, \quad t \in[0, T], \quad T<\infty .
$$

Рассмотрим также операторы $\tilde{K}$ и $\tilde{K}_{n}$, определенные формулами by (1.4.4) в (1.4.6) на пространствах

$$
\begin{aligned}
F & =C\left([0, T] ; E^{\theta}\right) \equiv\left\{u(t):\|u\|_{F}=\max _{t \in[0, T]}\|u(t)\|_{E^{\theta}}<\infty\right\}, \\
F_{n} & =C\left([0, T] ; E_{n}^{\theta}\right) \equiv\left\{u_{n}(t):\left\|u_{n}\right\|_{F_{n}}=\max _{t \in[0, T]}\left\|u_{n}(t)\right\|_{E_{n}^{\theta}}<\infty\right\} .
\end{aligned}
$$

Теорема 1.4.2. Предположим, что резольвента $(\lambda I-A)^{-1}$ компактна для некоторого $\lambda \in$ $\rho(A)$ и функиия $f(\cdot)$ является достаточно гладкой. Тогда оператор $\tilde{K}$ компактен.

Доказательство. Оператор $\tilde{K}$, определенный формулой (1.4.4), компактен в пространстве $F$. Действительно, оператор

$$
\mathcal{F}_{\epsilon}\left(u^{k}\right)(t)=\exp (\epsilon A) \int_{0}^{t-\epsilon} \exp ((t-s-\epsilon) A) f\left(u^{k}(s)\right) d s
$$

отображает любое ограниченное множество функций $\left\{u^{k}(\cdot)\right\},\left\|u^{k}(\cdot)\right\|_{F} \leqslant C$, в компактное множество в $E$ для любых $t>0$ и $0<\epsilon<t$. Видим, что

$$
\left\|\mathcal{F}_{\epsilon}\left(u^{k}\right)(t)-\mathcal{F}\left(u^{k}\right)(t)\right\| \leqslant C \epsilon
$$


для любого $t \in(0, T]$, где

$$
\mathcal{F}\left(u^{k}\right)(t)=\int_{0}^{t} \exp ((t-s) A) f\left(u^{k}(s)\right) d s
$$

и $0<\epsilon<t$. Отсюда следует, что оператор $\mathcal{F}(\cdot)(t): F \rightarrow E^{\theta}$ компактен для любого $t>0$. При $t=0$ оператор $\mathcal{F}(\cdot)(0)$ также компактен. Кроме того, множество функций $\left\{F_{k}(\cdot)\right\}, F_{k}(t)=\mathcal{F}\left(u^{k}\right)(t)$, $t \in[0, T]$, является эквиограниченным и равностепенно непрерывным семейством, поскольку для $0<t_{1}<t_{2}$ получим

$$
\left\|F_{k}\left(t_{2}\right)-F_{k}\left(t_{1}\right)\right\|_{E^{\theta}} \leqslant C\left(\int_{0}^{t_{1}}\left\|\exp \left(\left(t_{2}-s\right) A\right)-\exp \left(\left(t_{1}-s\right) A\right)\right\|_{E^{\theta}} d s+\left|t_{2}-t_{1}\right|^{1-\theta}\right)
$$

и $\exp (\cdot A)$ равномерно непрерывна при $t>0$. По обобщенной теореме Арцела-Асколи, аналогично теореме 1.4 .3 , заключаем, что оператор $\tilde{K}$ компактен.

Предложение 1.4.1. Предположим, что условия $(\mathrm{A})$ и $\left(\mathrm{B}_{1}\right)$ выполнены. Тогда компактная сходимость резольвент

$$
\left(\lambda I_{n}-A_{n}\right)^{-1} \stackrel{\mathcal{P P}}{\longrightarrow}(\lambda I-A)^{-1}
$$

эквивалентна компактной сходимости аналитических $C_{0}$-полугрупп $\exp \left(t A_{n}\right) \stackrel{\mathcal{P P}}{\longrightarrow} \exp (t A)$ для любого $t>0$.

Доказательство. Во-первых, покажем, что компактная сходимость резольвент (1.4.7) эквивалентна компактной сходимости $C_{0}$-полугрупп $\exp \left(t A_{n}\right) \rightarrow \exp (t A)$ для любого $t>0$. Пусть $\left\|x_{n}\right\|=O(1)$. Тогда из оценки

$$
\left\|A_{n} \exp \left(t A_{n}\right)\right\| \leqslant \frac{M}{t} e^{\omega t}
$$

получим ограниченность последовательности $\left\{\left(A_{n}-\lambda I_{n}\right) \exp \left(t A_{n}\right) x_{n}\right\}$. В силу теоремы 2.3.1 и компактной сходимости резольвент получаем компактность последовательности $\left\{\exp \left(t A_{n}\right) x_{n}\right\}$.

Необходимость будет доказана, если для меры некомпактности $\mu(\cdot)$ мы установим, что

$$
\mu\left(\left\{\left(\lambda I_{n}-A_{n}\right)^{-1} x_{n}\right\}\right)=0 \quad \text { при }\left\|x_{n}\right\|=O(1) .
$$

Имеем

$$
\begin{array}{r}
\mu\left(\left\{\left(\lambda I_{n}-A_{n}\right)^{-1} x_{n}\right\}\right)=\mu\left(\left\{\int_{0}^{\infty} e^{-\lambda t} \exp \left(t A_{n}\right) x_{n}\right\}\right) \leqslant \mu\left(\left\{\int_{0}^{q} e^{-\lambda t} \exp \left(t A_{n}\right) x_{n} d t\right\}\right)+ \\
+\mu\left(\left\{\int_{Q}^{\infty} e^{-\lambda t} \exp \left(t A_{n}\right) x_{n} d t\right\}\right)+\mu\left(\left\{\exp \left(\epsilon A_{n}\right) \int_{q}^{Q} e^{-\lambda t} \exp \left((t-\epsilon) A_{n}\right) x_{n} d t\right\}\right)
\end{array}
$$

Два первых члена справа можно сделать малыми (меньше, чем $\epsilon$ ) надлежащим выбором $q$ и $Q$. Последний член обращается в нуль благодаря компактной сходимости $\exp \left(\epsilon A_{n}\right) \stackrel{\mathcal{P} \mathcal{P}}{\longrightarrow} \exp (\epsilon A)$ для любого $0<\epsilon<q$.

Теорема 1.4.3. Операторная сходимость $\tilde{K}_{n} \rightarrow \tilde{K}$ компактна.

Доказательство. Чтобы показать, что $\tilde{K}_{n} \rightarrow \tilde{K}$ компактно, предположим, что $\left\|u_{n}\right\| F_{n}=$ $O(1)$. Тогда последовательность $\left\{\tilde{K}_{n} u_{n}\right\}$ является $\mathcal{P}$-компактной по обобщеннной теореме Арцела-Асколи. Чтобы это доказать, проверим, что мера некомпактности обращается в нуль, $\mu\left(\left\{\left(\tilde{K}_{n} u_{n}\right)(t)\right\}\right)=0$, для всех $t \in[0, T]$. Рассмотрим соотношение

$$
\left(\tilde{K}_{n} u_{n}\right)(t)=\exp \left(t A_{n}\right) u_{n}^{0}+\psi_{n}^{\tau}(t)+\varphi_{n}^{\tau}(t)
$$


где

$$
\begin{aligned}
& \psi_{n}^{\tau}(t)=\exp \left(\tau A_{n}\right) \int_{0}^{t-\tau} \exp \left((t-s-\tau) A_{n}\right) f_{n}\left(u_{n}(s)\right) d s \\
& \varphi_{n}^{\tau}(t)=\int_{t-\tau}^{t} \exp \left((t-s) A_{n}\right) f_{n}\left(u_{n}(s)\right) d s .
\end{aligned}
$$

В силу ограниченнности величины $\left\|f_{n}\left(u_{n}(\cdot)\right)\right\|_{F_{n}}$, можно выбрать $\left\|\varphi_{n}^{\tau}(\cdot)\right\|_{F_{n}}$ достаточно малым при достаточно малых $\tau$ и $\mu\left(\left\{\psi_{n}^{\tau}\right\}\right)=0$. Последовательность $\left\{e^{t A_{n}} u_{n}^{0}\right\}$ также $\mathcal{P}$-компактна.

Ясно, что $\left\{\tilde{K}_{n} u_{n}\right\}$ равномерно ограничена по $n$. Докажем равностепенную непрерывность. Для $t_{1} \leqslant t_{2}$ имеем

$$
\begin{aligned}
& \left\|A_{n}^{\theta} \int_{0}^{t_{2}} \exp \left(\left(t_{2}-s\right) A_{n}\right) f_{n}\left(u_{n}(s)\right) d s-A_{n}^{\theta} \int_{0}^{t_{1}} \exp \left(\left(t_{1}-s\right) A_{n}\right) f_{n}\left(u_{n}(s)\right) d s\right\|_{E_{n}} \leqslant \\
& \leqslant\left\|\int_{t_{1}}^{t_{2}} A_{n}^{\theta} e^{\left(t_{2}-s\right) A_{n}} f_{n}\left(u_{n}(s)\right) d s\right\|_{E_{n}}+\left\|\int_{0}^{t_{1}} A_{n}^{\theta}\left(e^{\left(t_{2}-s\right) A_{n}}-e^{\left(t_{1}-s\right) A_{n}}\right) f_{n}\left(u_{n}(s)\right) d s\right\|_{E_{n}} .
\end{aligned}
$$

Первый член стремится к нулю при $\left|t_{2}-t_{1}\right| \rightarrow 0$, поскольку

$$
\int_{t_{1}}^{t_{2}} \frac{d s}{\left(t_{2}-s\right)^{\theta}}=\left.\left(t_{2}-s\right)^{1-\theta}\right|_{t_{1}} ^{t_{2}}=\left(t_{2}-t_{1}\right)^{1-\theta} .
$$

Чтобы оценить второй член для любого $\epsilon>0$ и некоторого $0<\delta \leqslant \epsilon^{1 /(1-\theta)}$, рассмотрим случаи $t_{1} \leqslant \delta$ и $t_{1}>\delta$ отдельно. Если $t_{1} \leqslant \delta$ и $\left|t_{2}-t_{1}\right| \leqslant \delta$, то

$$
\left\|\int_{0}^{t_{2}} \cdots d s\right\| \leqslant c \delta^{1-\theta} \leqslant C \epsilon .
$$

Если $t_{1}>\delta$, то можно разложить второй член следующим образом:

$$
\int_{0}^{t_{1}}=\int_{0}^{t_{1}-\delta}+\int_{t_{1}-\delta}^{t_{1}}
$$

Таким образом, если $\left|t_{2}-t_{1}\right| \leqslant \delta$, то

$$
\int_{t_{1}-\delta}^{t_{1}} \leqslant c \delta^{1-\theta} \leqslant C \epsilon,
$$

как и ранее. Поскольку $t_{1}-s, t_{2}-s \in\left[\delta, t_{1}\right]$ при $s \in\left[0, t_{1}-\delta\right]$, то мы видим, что

$$
\int_{0}^{t_{1}} A_{n}^{\theta}\left(e^{\left(t_{2}-s\right) A_{n}}-e^{\left(t_{1}-s\right) A_{n}}\right) f_{n}\left(u_{n}(s)\right) d s \rightarrow 0
$$

при $\left|t_{2}-t_{1}\right| \rightarrow 0$ в силу сходимости

$$
\left\|A_{n}^{\theta}\left(e^{\left(t_{2}-s\right) A_{n}}-e^{\left(t_{1}-s\right) A_{n}}\right)\right\|_{E_{n}} \rightarrow 0
$$

при $\left|t_{2}-t_{1}\right| \rightarrow 0$, так как аргументы $t_{2}-s$ и $t_{1}-s$ отделены от нуля на расстояние, большее $\delta$. 
Теорема 1.4.4 (см. [26]). Предположим, что условия (A) и (B $\left.{ }_{1}\right)$ выполнены и компактные резольвенты $R(\lambda ; A)$ и $R\left(\lambda ; A_{n}\right)$ сходлтся компактно для некоторого $\lambda \in \rho(A)$ :

$$
\left(\lambda I_{n}-A_{n}\right)^{-1} \stackrel{\mathcal{P P}}{\longrightarrow}(\lambda I-A)^{-1} .
$$

Предположим также, что выполнены условия (A) u $\left(\mathrm{B}_{1}\right)$, условия гладкости для $f_{n} u f, a$ также условие

$$
f_{n}\left(x_{n}\right) \stackrel{\mathcal{P}}{\longrightarrow} f(x) \quad \text { npu } x_{n} \stackrel{\mathcal{P}^{\theta}}{\longrightarrow} x .
$$

Тогда для решений задач (1.4.4) и (1.4.6) имеем

$$
u_{n}\left(t, u_{n}^{0}\right) \stackrel{\mathcal{P}^{\theta}}{\longrightarrow} u\left(t, u^{0}\right) \quad \text { равномерно по } t \in[0, T], T<\infty, \text { при } u_{n}^{0} \stackrel{\mathcal{P}^{\theta}}{\longrightarrow} u^{0} .
$$

Доказательство. Известно (см. теорему 1.2.2), что $\operatorname{ind}\left(u^{*}, I-\tilde{K}\right)=1$. По теореме 1.4 .3 имеем компактную операторную сходимость $\tilde{K}_{n} \rightarrow \tilde{K} ;$ следовательно, согласно [259, теорема 3],

$$
\gamma\left(I_{n}-\tilde{K}_{n}, \partial \Omega_{n}\right)=1 \quad \text { при } n \geqslant n_{0} .
$$

Это означает, что найдется последовательность $\left\{u_{n}^{*}\right\}, n \in \mathbb{N}$, решений задач (1.4.6), причем $\left\{u_{n}^{*}\right\}$, $u_{n}^{*} \in F_{n}$, компактна. Таким образом, заключаем, что $u_{n}^{*}(t) \stackrel{\mathcal{P}^{\theta}}{\longrightarrow} u^{*}(t)$ равномерно по $t \in[0, T]$ при $n \rightarrow \infty$.

\section{ГЛАВА 2}

\section{СХОДИМОСТЬ АТТРАКТОРОВ}

Интерес к изучению существования и аппроксимации аттракторов не ослабевает (см. $[15,37,67,78$, $97,100,139,237,278,279])$. Хорошо известен стандартный результат теории динамических систем, согласно которому аттракторы параметрических динамических систем сходятся полунепрерывно сверху по параметру (см. $[88,132])$. При дополнительных предположениях имеет место полунепрерывная снизу сходимость и, следовательно, непрерывная сходимость аттракторов. Такие предположения обычно связаны со структурой аттракторов, например структурой Морса-Смейла. Имеется способ (см. [174]) установить, что непрерывная сходимость аттракторов эквивалентна их свойству равномерного притяжения (см. также [20], где аналогичный результат был анонсирован без доказательства и не на общей аппроксимационной схеме).

Аналогичный результат о полунепрерывной сверху сходимости имеет место для численных аппроксимаций обыкновенных дифференциальных уравнений (см. $[53,134,157,243])$ и для галеркинских аппроксимаций параболических дифференциальных уравнений с частными производными (см. [158]). Сходимость аттракторов неконформных конечно-элементных аппроксимаций параболических уравнений с частными производными и зависимость аттракторов дифференциальных уравнений с запаздыванием более сложны для исследования, поскольку сравниваемые системы имеют различные пространства состояний, однако факт аналогичной полунепрерывной сверху сходимости можно установить (см. $[156,196])$.

В этой главе мы на основе концепции «дискретной сходимости», определенной в главе 1 для сравнения систем на различных пространствах, докажем эквивалентность свойства равномерного притяжения и непрерывной сходимости аттракторов таких систем. Этот результат будет применен к общим аппроксимационным схемам для абстрактных полулинейных параболическых систем, включая конечно-разностные и неконформные конечно-элементные аппроксимации, а также аппроксимации Галеркина и конформные конечно-элементные аппроксимации. 


\section{1. ДИСКРЕТНАЯ СХОДИМОСТЬ мНОЖЕСТВ}

Для непустых подмножеств $U_{n}$ и $V_{n}$ пространств $E_{n}, n \in \mathbb{N}$, и непустого подмножества $U$ пространства $E$ введем полуметрику Хаусдорфа

и метрику Хаусдорфа

$$
d_{n}\left(U_{n}, V_{n}\right)=\sup _{u_{n} \in U_{n}} \inf _{v_{n} \in V_{n}}\left\|u_{n}-v_{n}\right\|_{E_{n}}
$$

$$
\delta_{n}\left(U_{n}, U\right) \equiv \delta_{n}\left(U, U_{n}\right)=\max \left\{d_{n}\left(p_{n} U, U_{n}\right), d_{n}\left(U_{n}, p_{n} U\right)\right\} .
$$

Отметим, что $\delta_{n}\left(U_{n}, U\right)$ может обращаться в нуль и в случае, когда $U_{n} \neq U$.

Определение 2.1.1. Пусть $\mathcal{A}_{n} \subset E_{n}, n \in \mathbb{N}$, и $\mathcal{A} \subset E$.

(1) Будем говорить, что семейство множеств $\left\{\left\{\mathcal{A}_{n}\right\}, n \in \mathbb{N}\right\}$ сходится $\mathcal{P}$-полунепреръвно сверху к множеству $\mathcal{A} \subset E$, если $d_{n}\left(\mathcal{A}_{n}, p_{n} \mathcal{A}\right) \rightarrow 0$ при $n \rightarrow \infty$.

(2) Будем говорить, что семейство множеств $\left\{\left\{\mathcal{A}_{n}\right\}, n \in \mathbb{N}\right\}$ сходится $\mathcal{P}$-полунепрерывно снизу к множеству $\mathcal{A} \subset E$, если $d_{n}\left(p_{n} \mathcal{A}, \mathcal{A}_{n}\right) \rightarrow 0$ при $n \rightarrow \infty$.

Объединяя эти понятия, скажем, что $\mathcal{A}_{n}$ сходится $\mathcal{P}$-непрерывно к $\mathcal{A} \subset E$ при $n \rightarrow \infty$, если

$$
\delta_{n}\left(\mathcal{A}_{n}, p_{n} \mathcal{A}\right) \rightarrow 0 \quad \text { при } n \rightarrow \infty .
$$

Лемма 2.1.1 (см. [89]). Пусть $\mathcal{A}_{n} \subset E_{n}, n \in \mathbb{N}, u \mathcal{A} \subset E$.

(1) Если любая последовательность $\left\{u_{n}\right\}, u_{n} \in \mathcal{A}_{n}$, обладает $\mathcal{P}$-сходящейся подпоследовательностью, предел которой принадлежит $\mathcal{A}$, то $\mathcal{A}_{n}$ сходится $\mathcal{P}$-полунепрерывно сверху $\kappa \mathcal{A}$.

(2) Если семейство $\mathcal{A}$ компактно и для любого $u \in \mathcal{A}$ найдется последовательность $\left\{u_{n}\right\}$, $u_{n} \in \mathcal{A}_{n}, \mathcal{P}$-сходящался $к и$, то $\mathcal{A}_{n}$ сходится $\mathcal{P}$-полунепрерывно снизу $\kappa \mathcal{A}$.

\section{2. СВОЙСТВО РАВНОМЕРНОГО ПРИТЯЖЕНИЯ И НЕПРЕРЫВНАЯ СХОДИМОСТЬ}

Полудинамическая система $S_{n}(\cdot)$ на банаховом пространстве $\left(E_{n},\|\cdot\|_{E_{n}}\right)$ называется диссиnативной (см. [159]), если найдется такое ограниченное подмножество $U_{n}$ пространства $E_{n}$, что для любых ограниченных подмножеств $B_{n}$ пространств $E_{n}$ существуют конечные времена $T_{n}=T_{n}\left(B_{n}\right)$, для которых

$$
S_{n}(t) B_{n} \subseteq U_{n} \quad \text { при } t \geqslant T_{n} .
$$

Определение 2.2.1. Будем говорить, что семейство полудинамических систем $\left\{S_{n}(\cdot)\right\}, n \in \mathbb{N}$, является $\mathcal{P}$-эквидиссипативным, если найдется такая равномерно ограниченная последовательность $\left\{U_{n}\right\}, n \in \mathbb{N}$, подмножеств $U_{n}$ пространств $E_{n}$, что для любой равномерно ограниченной последовательности $\mathcal{B}=\left\{\left\{B_{n}\right\}, n \in \mathbb{N}\right\}$ подмножеств $B_{n} \subset E_{n}$ существует конечное $T_{0}=T_{0}(\mathcal{B})$, не зависящее от $n$, для которого

$$
S_{n}(t) B_{n} \subseteq U_{n} \quad \text { при } t \geqslant T_{0} .
$$

Если полудинамическая система $S_{n}(\cdot)$ имеет глобальный аттрактор $\mathcal{A}_{n}$ при любом $n \in \mathbb{N}$, то $\mathcal{A}_{n}$ инвариантно, т.е. $S_{n}(t) \mathcal{A}_{n}=\mathcal{A}_{n}$ для всех $t \geqslant 0$, и $\mathcal{A}_{n}$ притягивает произвольные ограниченные подмножества пространства $E_{n}$ под действием $S_{n}(\cdot)$, т.е. для любого ограниченного подмножества $B_{n}$ в $E_{n}$ и любого $\epsilon>0$ существует такое $\tau_{n}=\tau_{n}\left(B_{n}, \varepsilon\right)>0$, что

$$
d_{n}\left(S_{n}(t) B_{n}, \mathcal{A}_{n}\right)<\varepsilon \quad \forall t \geqslant \tau_{n} .
$$

Определение 2.2.2. Будем говорить, что семейство аттракторов $\left\{\mathcal{A}_{n}\right\}, n \in \mathbb{N}$, семейства полудинамических систем $\left\{S_{n}(\cdot)\right\}, n \in \mathbb{N}$, обладает свойством $\mathcal{P}$-равномерного притяљсения, если для любой равномерно ограниченной последовательности $\mathcal{B}=\left\{B_{n}, n \in \mathbb{N}\right\}$ подмножеств $B_{n}$ в $E_{n}$ и любого $\epsilon>0$ существует такое конечное $T=T(\mathcal{B}, \epsilon)>0$, не зависящее от $n$, что

$$
d_{n}\left(S_{n}(t) B_{n}, \mathcal{A}_{n}\right)<\epsilon \quad \text { для любого } t \geqslant T .
$$

Наложим следующее условие сходимости для семейства $\left\{S_{n}(\cdot)\right\}, n \in \mathbb{N}$, полудинамических систем в банаховых пространствах $E_{n}$ и полудинамических систем $S(\cdot)$ в банаховых пространствах $E$. 
Предположение 2.2.1. Семейство полудинамических систем $\left\{S_{n}(\cdot)\right\}, n \in \mathbb{N}$, дискретно компактно сходится к полудинамической системе $S(\cdot)$, т.е. $S_{n}(t) \stackrel{\mathcal{P} \mathcal{P}}{\longrightarrow} S(t)$ для любого $t \geqslant 0$ и любой равномерно ограниченной последовательности $\left\{x_{n}\right\}, n \in \mathbb{N}$, и последовательность $\left\{S_{n}(t) x_{n}\right\}$, $n \in \mathbb{N}, \mathcal{P}$-компактна для любого $t>0$.

Сформулируем основной результат этой главы.

Теорема 2.2.2 (см. [159]). Предположим, что семейство полудинамических систем $\left\{S_{n}(\cdot)\right\}$, $n \in \mathbb{N}$, в банаховых пространствах $E_{n}$ является $\mathcal{P}$-эквидиссипативным. Кроме того, предположим, что $S(\cdot)$ - такая полудинамическая система в банаховом пространстве E, что предположение 2.2 .1 имеет место для $S_{n}(\cdot)$ и $S(\cdot)$. Пусть $\mathcal{A}_{n}$ - глобальный аттрактор для $S_{n}(\cdot)$ u $\mathcal{A}$ - глобальный аттрактор для $S(\cdot)$. Семейство аттракторов $\left\{\mathcal{A}_{n}\right\}, n \in \mathbb{N}$, обладает свойством $\mathcal{P}$-равномерного притяљсения тогда и только тогда, когда $\mathcal{A}_{n}$ сходится $\mathcal{P}$-непрерывно $\kappa$ глобальному аттрактору $\mathcal{A}$.

Доказательство. Диссипативность и компактность обеспечивают существование глобальных аттракторов и, кроме того, равномерную ограниченность последовательности аттракторов $\mathcal{A}_{n}$.

Во-первых, покажем $\mathcal{P}$-полунепрерывную сверху сходимость. Предположим, что $x_{n}^{*} \stackrel{\mathcal{P}}{\longrightarrow} x^{*}$ и $x_{n}^{*} \in \mathcal{A}_{n}$. Поскольку $S_{n}(t) \mathcal{A}_{n}=\mathcal{A}_{n}$ для любого $t>0$, найдется такая последовательность $\left\{x_{n}\right\}$, $x_{n} \in \mathcal{A}_{n}$, что $S_{n}(t) x_{n}=x_{n}^{*}$ для любого $t>0$. Множество $\left\{x_{n}\right\}$ ограничено в силу $\mathcal{P}$-эквидиссипативности; кроме того, $\left\{x_{n}\right\} \mathcal{P}$-компактна. Таким образом, существует такое подмножество $\mathbb{N}^{\prime} \subset \mathbb{N}$, что $x_{n} \stackrel{\mathcal{P}}{\longrightarrow} x$ при $n \rightarrow \infty$ в $\mathbb{N}^{\prime}$ для некоторого $x \in E$. Благодаря единственности предела ясно, что $S_{n}(T) x_{n} \stackrel{\mathcal{P}}{\longrightarrow} S(T) x=x^{*}$ при $n \rightarrow \infty$ в $\mathbb{N}^{\prime}$. Из равномерной ограниченности $S_{n}(T) x_{n}$ следует, что для любого $\epsilon>0$ найдется такое $T(\epsilon)>0$, что $S(T) x$ лежит в некотором ограниченном шаре и

$$
d_{E}(S(T) x, \mathcal{A})<\epsilon \quad \text { для всех } T>T(\epsilon) .
$$

Поскольку $S(T) x=x^{*}$, можно выбирать $x^{*}$ все ближе и ближе к $\mathcal{A}$, полагая $T$ достаточно большим. Поскольку

$$
d_{n}\left(x_{n}^{*}, p_{n} \mathcal{A}\right) \leqslant d_{n}\left(S_{n}(T) x_{n}, p_{n} S(T) x\right)+d_{n}\left(p_{n} S(T) x, p_{n} \mathcal{A}\right),
$$

заключаем, что $d_{n}\left(x_{n}^{*}, p_{n} \mathcal{A}\right) \rightarrow 0$ при $n \rightarrow \infty$ in $\mathbb{N}^{\prime}$. Таким образом, $\mathcal{A}_{n}$ сходится $\mathcal{P}$-полунепрерывно сверху к $\mathcal{A}$.

Пусть теперь семейство аттракторов $\left\{\mathcal{A}_{n}\right\}, n \in \mathbb{N}$, обладает свойством $\mathcal{P}$-равномерного притяжения. Предположим, что $\mathcal{A}_{n}$ не сходится $\mathcal{P}$-полунепрерывно снизу к $\mathcal{A}$. Тогда существует такая последовательность $x^{n} \in \mathcal{A}$, что

$$
d_{n}\left(p_{n} x^{n}, \mathcal{A}_{n}\right) \geqslant 1>0, \quad n \in \mathbb{N}
$$

Поскольку $\mathcal{A}$ компактно, найдется такое подмножество $\mathbb{N}^{\prime} \subset \mathbb{N}$, что $x^{n} \rightarrow x^{*}$ при $n \rightarrow \infty$ в $\mathbb{N}^{\prime}$. Следовательно,

$$
d_{n}\left(p_{n} x^{n}, \mathcal{A}_{n}\right) \leqslant d_{n}\left(p_{n} x^{*}, \mathcal{A}_{n}\right)+\frac{\epsilon}{3}
$$

при $n \geqslant N(\epsilon)$ в $\mathbb{N}^{\prime}$. Благодаря $S(T)$-инвариантности аттрактора $\mathcal{A}$ найдется такой элемент $x \in \mathcal{A}$, что $S(T) x=x^{*}$.

Согласно предположению 2.2 .1 найдется такая последовательность $x_{n} \in E_{n}$, что $x_{n} \stackrel{\mathcal{P}}{\longrightarrow} x$, причем $S_{n}(T) x_{n} \stackrel{\mathcal{P}}{\longrightarrow} S(T) x$ при $n \rightarrow \infty$. Поскольку $\mathcal{A}$ ограничен, последовательность $\left\{x_{n}\right\}$ также содержится в последовательности равномерно ограниченных шаров $\mathcal{B}=\left\{\left\{B_{n}\right\}, n \in \mathbb{N}\right\}$, т.е. $x_{n} \in B_{n}$ для каждого $n$. Согласно свойству $\mathcal{P}$-равномерного притяжения для этой равномерно ограниченной последовательности $\mathcal{B}$ существует такое $T=T(\mathcal{B}, \epsilon / 3)$, что $d_{n}\left(S_{n}(T) B_{n}, \mathcal{A}_{n}\right) \leqslant \epsilon / 3$. Окончательно для $T=T(\mathcal{B}, \epsilon / 3)$ получим

$$
d_{n}\left(p_{n} x^{n}, \mathcal{A}_{n}\right) \leqslant d_{n}\left(p_{n} x^{*}, \mathcal{A}_{n}\right)+\frac{\epsilon}{3} \leqslant\left\|p_{n} S(T) x-S_{n}(T) x_{n}\right\|_{E_{n}}+d_{n}\left(S_{n}(T) x_{n}, \mathcal{A}_{n}\right)+\frac{\epsilon}{3} \leqslant \epsilon
$$


при достаточно больших $n \in \mathbb{N}^{\prime}$, зависящих от $\epsilon$. Выбирая $\epsilon<1$, получим противоречие с (2.2.3). Таким образом, $\mathcal{P}$-полунепрерывная снизу сходимость аттракторов имеет место, откуда получаем их $\mathcal{P}$-непрерывную сходимость.

Теперь предположим, что $\mathcal{A}_{n}$ сходятся $\mathcal{P}$-непрерывно к $\mathcal{A}$, т.е. $\delta_{n}\left(\mathcal{A}_{n}, \mathcal{A}\right) \rightarrow 0$ при $n \rightarrow \infty$. Докажем от противного, что они обладают свойством $\mathcal{P}$-равномерного притяжения. Предположим, что для некоторой равномерно ограниченной последовательности множеств $\left\{B_{n}\right\}, n \in \mathbb{N}$, имеем

$$
d_{n}\left(S_{n}(T) B_{n}, \mathcal{A}_{n}\right) \geqslant 1>0
$$

для любого $T>0$ равномерно по $n \in \mathbb{N}^{\prime}$. Тогда найдется такая последовательность $\left\{u_{n}\right\}$, $u_{n} \in B_{n}$, что $d_{n}\left(S_{n}(T) u_{n}, \mathcal{A}_{n}\right) \geqslant 1>0$ равномерно по $n \in \mathbb{N}^{\prime}$ для любого $T>0$. Ясно, что последовательность $\left\{S_{n}\left(t_{0}\right) u_{n}\right\}$ является $\mathcal{P}$-компактной для некоторого $t_{0}>0$ и, кроме того, что она сходится на некоторой подпоследовательности $n \in \mathbb{N}^{\prime \prime}, S_{n}\left(t_{0}\right) u_{n} \stackrel{\mathcal{P}}{\longrightarrow} u^{*}$. Таким образом, $S_{n}(T) u_{n} \stackrel{\mathcal{P}}{\longrightarrow} S\left(T-t_{0}\right) u^{*}$ на некоторой подпоследовательности $n \in \mathbb{N}^{\prime \prime}$, где $\mathbb{N}^{\prime \prime} \subset \mathbb{N}^{\prime}$. Теперь $d\left(S\left(T-t_{0}\right) u^{*}, \mathcal{A}\right)$ можно выбрать произвольно малым для произвольно большого $T-t_{0}$. Следовательно,

$$
d_{n}\left(S_{n}(T) u_{n}, \mathcal{A}_{n}\right) \leqslant d_{n}\left(S_{n}(T) u_{n}, p_{n} S\left(T-t_{0}\right) u^{*}\right)+d_{n}\left(p_{n} S\left(T-t_{0}\right) u^{*}, p_{n} \mathcal{A}\right)+d_{n}\left(p_{n} \mathcal{A}, \mathcal{A}_{n}\right)
$$

можно сделать меньше 1 при $n \in \mathbb{N}^{\prime \prime}$ и достаточно большом $T$; это противоречит неравенству (2.2.4). Отсюда следует, что семейство аттракторов $\left\{\mathcal{A}_{n}\right\}, n \in \mathbb{N}$, обладает свойством $\mathcal{P}$ равномерного притяжения. Теорема 2.2 .2 доказана.

\section{3. АБСТРАКТНЫЕ ПАРАБОЛИЧЕСКИЕ ЗАДАЧИ}

В этом разделе мы рассмотрим применения теоремы 2.2 .2 к абстрактным полулинейным параболическим задачам.

Предположим, что $\theta \in(0,1)$, оператор $A$ удовлетворяет условию $(1.1 .3)$ и $f(\cdot): E^{\theta} \rightarrow E-$ глобально липшицева, ограниченная и непрерывно дифференцируемая по Фреше функция. Тогда полулинейная автономная параболическая начальная задача (1.2.16) имеет единственное обобщенное решение, определенное для всех $t \geqslant 0$, и нелинейные операторы $u(\cdot)=S(\cdot) u^{0}: \overline{\mathbb{R}}^{+} \rightarrow E^{\theta}$ образуют сильно непрерывную полугруппу операторов на $E^{\theta}$, т.е. полудинамическую систему (см. $[136,262])$. Кроме того, полугруппа $S(\cdot)$ задается формулой вариации постоянной:

$$
S(t) u^{0}=\exp \left(\left(t-t_{0}\right) A\right) S\left(t_{0}\right) u^{0}+\int_{t_{0}}^{t} \exp ((t-s) A) f\left(S(s) u^{0}\right) d s, \quad t \geqslant t_{0},
$$

и является эквидиссипативной и компактной. Таким образом, она обладает глобальным аттрактором $\mathcal{A}$ в $E^{\theta}$ (см. $\left.[89,91]\right)$.

Рассмотрим замкнутые линейные операторы $A_{n}: D\left(A_{n}\right) \subseteq E_{n} \rightarrow E_{n}, n \in \mathbb{N}$, резольвенты которых удовлетворяют неравенству

$$
\left\|\left(\lambda I_{n}-A_{n}\right)^{-1}\right\|_{B\left(E_{n}\right)} \leqslant \frac{M_{2}}{\left|\lambda-\omega_{2}\right|}, \quad \operatorname{Re} \lambda>\omega_{2}, \quad n \in \mathbb{N}
$$

для некоторых констант $M_{2} \geqslant 1$ и $\omega_{2}$ (см. условие $\left(\mathrm{B}_{1}\right)$ в теореме 1.3.1). Напомним (см. [26]), что из условия $\Delta_{c c} \neq \varnothing$ следует, что имеет место оценка $\left(\mathrm{B}_{1}\right)$ в виде

$$
\left\|\left(\lambda I_{n}-A_{n}\right)^{-1}\right\|_{B\left(E_{n}\right)} \leqslant \frac{M}{1+|\lambda|}, \quad \operatorname{Re} \lambda>0, \quad n \in \mathbb{N} .
$$

Следовательно, операторы $\left(-A_{n}\right)^{\theta}$ корректно определены. Позже нам также понадобятся операторы $p_{n}^{\theta}=\left(-A_{n}\right)^{-\theta} p_{n}(-A)^{\theta} \in B\left(E^{\theta}, E_{n}^{\theta}\right)$, обладающие тем же свойством (1.3.1), но в пространствах $E^{\theta}$ и $E_{n}^{\theta}$. Предположим, что операторы $A_{n}$ и $A$ связаны условиями $(1.1 .3),(\mathrm{A})$ и $\left(\mathrm{B}_{1}\right)$. Сходимость $y_{n} \stackrel{\mathcal{P}^{\theta}}{\longrightarrow} y$ определена по отношению к пространствам $E_{n}^{\theta}$ и $E^{\theta}$. 
Определение 2.3.1. Будем говорить, что последовательность замкнутых линейных операторов $\left\{A_{n}\right\}, A_{n} \in \mathcal{C}\left(E_{n}\right), n \in \mathbb{N}$, согласована с замкнутым линейным оператором $A \in \mathcal{C}(E)$, если для каждого $x \in D(A)$ найдется такая последовательность $\left\{x_{n}\right\}, x_{n} \in D\left(A_{n}\right) \subseteq E_{n}, n \in \mathbb{N}$, что $x_{n} \stackrel{\mathcal{P}}{\longrightarrow} x$ и $A_{n} x_{n} \stackrel{\mathcal{P}}{\longrightarrow} A x$. Кратко будем говорить, что $\left(A_{n}, A\right)$ согласованы.

Предположим, что операторы $\left(A_{n}, A\right)$ согласованы и $f_{n}(\cdot): E_{n}^{\theta} \rightarrow E_{n}$ - глобально ограниченные, равномерно глобально липшиц-непрерывные и непрерывно дифференцируемые по Фреше функции, $n \in \mathbb{N}$. Кроме того, предположим, что $f_{n}\left(x_{n}\right) \stackrel{\mathcal{P}}{\longrightarrow} f(x)$ и $f_{n}^{\prime}\left(x_{n}\right) \stackrel{\mathcal{P}^{\theta} \mathcal{P}}{\longrightarrow} f^{\prime}(x)$ при $x_{n} \stackrel{\mathcal{P}^{\theta}}{\longrightarrow} x$ и для любого $\rho>0$ существует такое $\delta>0$, что

$$
\sup _{n \in \mathbb{N}} \sup _{\left\|w_{n}\right\|_{E_{n}^{\theta}} \leqslant \delta}\left\|f_{n}^{\prime}\left(w_{n}+p_{n}^{\theta} u^{*}\right)-f_{n}^{\prime}\left(p_{n}^{\theta} u^{*}\right)\right\|_{B\left(E_{n}^{\theta}, E_{n}\right)} \leqslant \rho
$$

при всех $u^{*}$, где $A u^{*}+f\left(u^{*}\right)=0$.

В качестве аппроксимации начальной задачи (1.2.16) рассмотрим автономные начальные задачи

$$
\begin{aligned}
& u_{n}^{\prime}(t)=A_{n} u_{n}(t)+f_{n}\left(u_{n}(t)\right), \quad t \geqslant 0, \\
& u_{n}(0)=u_{n}^{0} \in E_{n}^{\theta},
\end{aligned}
$$

где $u_{n}^{0} \stackrel{\mathcal{P}^{\theta}}{\longrightarrow} u^{0}$. При наложенных выше условиях задача (2.3.2) имеет единственное обобщенн'мягк'ое решение $u_{n}(\cdot)=S_{n}(\cdot) u_{n}^{0}: \overline{\mathbb{R}}^{+} \rightarrow E_{n}^{\theta}$, которое порождает сильно непрерывную полугруппу $S_{n}(\cdot)$, заданную формулой вариации постоянной:

$$
S_{n}(t) u_{n}^{0}=\exp \left(\left(t-t_{0}\right) A_{n}\right) S_{n}\left(t_{0}\right) u_{n}^{0}+\int_{t_{0}}^{t} \exp \left((t-s) A_{n}\right) f_{n}\left(S_{n}(s) u_{n}^{0}\right) d s, \quad t \geqslant t_{0},
$$

при всех $n \in \mathbb{N}$. Кроме того, соответствующая полудинамическая система $S_{n}(\cdot)$ диссипативна и компактна и обладает глобальным аттрактором $\mathcal{A}_{n}$ в $E_{n}^{\theta}$.

Рассмотрим некоторые случаи, в которых теорему 2.2 .2 об эквивалентности свойства $\mathcal{P}$-равномерного притяжения и $\mathcal{P}$-непрерывной сходимости можно применить к указанной системе в пространствах $E_{n}^{\theta}$ и $E^{\theta}$. Для этой цели удобно сузить рассмотрение аппроксимационных операторов $A_{n}$ до операторов с компактными резольвентами.

По поводу определения множеств $\Delta_{s}, \Delta_{c}$ и $\Delta_{c c}$ см. раздел 3.2.2.

Теорема 2.3.1 (см. [26]). Предположим, что $\Delta_{c c} \neq \varnothing$. Тогда для любого $\zeta \in \Delta_{s}$ имеет место следующая импликачия:

$$
\left\|x_{n}\right\|_{E_{n}}=O(1) \&\left\|\left(\zeta I-A_{n}\right) x_{n}\right\|_{E_{n}}=O(1) \quad \Longrightarrow \quad\left\{x_{n}\right\} \text { является } \mathcal{P} \text {-компактной. }
$$

Обратно, если для некоторого $\zeta \in \Delta_{c} \cap \rho(A)$ имеет место импликация (2.3.4), то $\Delta_{c c} \neq \varnothing$.

Доказательство. Пусть $\left(\mu I_{n}-A_{n}\right)^{-1} \stackrel{\mathcal{P P}}{\longrightarrow}(\mu I-A)^{-1}$ компактно для некоторого $\mu \in \Delta_{c c}$. Тогда при $\left\|x_{n}\right\|_{E_{n}}=O(1)$ и $\left\|\left(\zeta I-A_{n}\right) x_{n}\right\|_{E_{n}}=O(1)$ из тождества Гильберта

$$
\left(\zeta I-A_{n}\right)^{-1}-\left(\mu I-A_{n}\right)^{-1}=(\mu-\zeta)\left(\zeta I-A_{n}\right)^{-1}\left(\mu I-A_{n}\right)^{-1}
$$

получим

$$
x_{n}=\left(\mu I-A_{n}\right)^{-1}\left(\zeta I-A_{n}\right) x_{n}-(\zeta-\mu)\left(\mu I-A_{n}\right)^{-1} x_{n} ;
$$

таким образом, последовательность $\left\{x_{n}\right\}$ является $\mathcal{P}$-компактной.

Обратно, пусть импликация (2.3.4) имеет место для некоторого $\zeta_{0} \in \Delta_{c} \cap \rho(A)$. Покажем, что $\zeta_{0} \in \Delta_{c c}$. Выбрав ограниченную последовательность $\left\{y_{n}\right\}, n \in \mathbb{N}$, получим

$$
\left\|\left(\zeta_{0} I-A_{n}\right)^{-1} y_{n}\right\|_{E_{n}}=O(1), \quad n \in \mathbb{N} .
$$

Применим импликацию (2.3.4) к последовательности $x_{n}=\left(\zeta_{0} I-A_{n}\right)^{-1} y_{n}$. Легко видеть, что последовательность $\left\{x_{n}\right\}$ является $\mathcal{P}$-компактной. Таким образом, $\zeta_{0} \in \Delta_{c c}$.

Сделаем следующее предположение. 
Предположение 2.3.2. Предположим, что выполнены резольвентные условия (1.1.3) и $\left(\mathrm{B}_{1}\right)$, а также условие $\Delta_{c c} \neq \varnothing$, резольвенты $\left(\lambda I_{n}-A_{n}\right)^{-1}$ компактны при $\lambda \in \Delta_{c c}$ и $f_{n}\left(x_{n}\right) \stackrel{\mathcal{P}}{\longrightarrow} f(x)$ при $x_{n} \stackrel{\mathcal{P}^{\theta}}{\longrightarrow} x$.

Теорема 2.3.3. Предположим, что условие 2.3.2 въполнено. Тогда $S_{n}(t) u_{n}^{0} \stackrel{\mathcal{P}^{\theta}}{\longrightarrow} S(t) u^{0}$ равномерно по $t \in[0, T], T<\infty$, при $u_{n}^{0} \stackrel{\mathcal{P}^{\theta}}{\longrightarrow} u^{0}$.

Нетрудно видеть, что при условии 2.3 .2 семейство операторов $S_{n}(t)$ сходится компактно к $S(t)$ для любого $t>0$, так что условие дискретной компактности из предположения 2.2.1 выполнено.

Отсылаем читателя к [89] (см. также ссылки в указанной работе), где подробно обсуждаются конкретные аппроксимации, использующие методы конечных элементов и конечно-разностные методы; в частности, показано, как использовать (2.3.4) для доказательства компактной сходимости резольвент. Также в [89] указан способ проверки предположения $\Delta_{c c} \neq \varnothing$, например, в случае метода конечных элементов.

Замечание 2.3.1 (о непрерывной сходимости и свойстве равномерного притяжения). Известно, что непрерывная сходимость аттракторов наблюдается в некоторых задачах, если исходный аттрактор является аттрактором Морса-Смейла, т.е. состоит из конечного числа гиперболических точек равновесия и их неустойчивых многообразий, хотя непрерывная сходимость также возможна и для негиперболических равновесий (см. [20,161]).

Рассмотрим особую ситуацию. Предположим, что траектория $S(\cdot) u^{0}$ исходной системы, начинающаяся в произвольной точке $u^{0}$. сходится к некоторому пределу, т.е. $S(t) u^{0} \rightarrow u^{*}$ при $t \rightarrow \infty$. Тогда точка $u^{*}$ (которая может зависеть от $u^{0}$ ) является решением уравнения $A u+f(u)=0$, т.е. является точкой равновесия. Такие равновесия существуют при определенных предположениях о функции $f(\cdot)$, и задачи $(2.3 .2)$ также обладают точками равновесия, которые близки к одноточечному множеству $\left\{u^{*}\right\}$, т.е. $u_{n}^{*} \stackrel{\mathcal{P}}{\longrightarrow} u^{*}$ при $n \rightarrow \infty$. В некотором смысле мы имеем непрерывность аттракторов в точке равновесия $\left\{u^{*}\right\}$. Предположим, что $a \in \mathcal{A}$ не является точкой равновесия. Тогда в $\epsilon$-окрестности $\mathcal{U}\left(u^{*}, \epsilon\right)$ точки $u^{*}$ существует такая точка $a^{\prime}$, что $S(T) a^{\prime}=a$ при некотором $T>0$. Следуя [89], можем найти такие точки $a_{n}^{\prime} \in \mathcal{A}_{n} \cap \mathcal{U}\left(u_{n}^{*}, \epsilon\right)$, что $a_{n}^{\prime} \stackrel{\mathcal{P}^{\theta}}{\longrightarrow} a^{\prime}$ и, согласно предположению сходимости 2.2.1, имеем $a_{n}=S_{n}(T) a_{n}^{\prime} \stackrel{\mathcal{P}^{\theta}}{\longrightarrow} S(T) a^{\prime}=a$. Поскольку $a_{n}^{\prime} \in \mathcal{A}_{n}$, отсюда следует, что $a_{n} \in \mathcal{A}_{n}$, и непрерывность аттракторов доказана. По теореме 2.2 .2 можем теперь заключить, что нелинейные полугруппы $S_{n}(\cdot)$ обладают свойством $\mathcal{P}$-равномерного притяжения

\section{ГЛАВА 3}

\section{СХОДИМОСТЬ АТТРАКТОРОВ В ГИПЕРБОЛИЧЕСКОМ СЛУЧАЕ}

Пусть $E$-комплексное банахово пространство и $A: D(A) \subset E \rightarrow E$-замкнутый линейный оператор с компактной резольвентой, для которого имеет место условие (1.1.3). В этой ситуации

$$
\operatorname{Re} \sigma(A)=\{\operatorname{Re} \lambda: \lambda \in \sigma(A)\} \subset(-\infty, 0) .
$$

Обозначим через $(-A)^{\theta}, \theta \in \overline{\mathbb{R}}^{+}$, операторы дробных степеней, ассоциированные с $A$, а через $E^{\theta}-$ пространства дробных степеней, ассоциированные с $A$. Хорошо известно (см. [136]), что при этих предположениях найдутся такие константы $M, \beta>0$, что

$$
\left\|e^{t A}\right\|_{B\left(E^{\theta_{1}}, E^{\theta_{2}}\right)} \leqslant M t^{\theta_{1}-\theta_{2}} e^{-\beta t}, \quad \theta_{2}>\theta_{1} \geqslant 0, \quad t \geqslant 0 .
$$

При сделанных предположениях, а также условии, что функция $f(\cdot): E^{\theta} \rightarrow E$ глобально ограничена, равномерно глобально липшиц-непрерывна и непрерывно дифференцируема по Фреше, глобально определено обобщенное решение задачи (1.2.16) (см. [136]). Пусть 
$u(t)=S(\cdot) u^{0}: \overline{\mathbb{R}}^{+} \rightarrow E^{\theta}$ - обобщенное решение задачи (1.2.16). Тогда можно определить семейство нелинейных операторов $\{S(t): t \geqslant 0\}$, обладающее следующими свойствами:

(i) $S(0)=I$,

(ii) $S(t+s)=S(t) S(s), t \geqslant 0, s \geqslant 0$,

(iii) отображение $\overline{\mathbb{R}}^{+} \times E^{\theta} \ni(t, x) \mapsto S(t) x \in E^{\theta}$ непрерывно.

Хорошо известно, что нелинейная полугруппа $S(\cdot)$ определяется формулой вариаии постоянной (2.3.1). Для каждого $u^{0} \in E^{\theta}$ определена положительная орбита $\gamma^{+}\left(u^{0}\right)$, проходящая через $u^{0}$ : $\gamma^{+}\left(u^{0}\right)=\left\{S(t) u^{0}: t \geqslant 0\right\}$. Отрицательная орбита, проходящая через $u^{0}$-это такая непрерывная функция $\phi:(-\infty, 0] \rightarrow E^{\theta}$, что $\phi(0)=u^{0}$ и для любого $s \leqslant 0$ выполняется соотношение $S(t) \phi(s)=\phi(t+s)$ при $0 \leqslant t \leqslant-s$. Полная орбита, проходящая через $u^{0}-$ это такая функция $\phi: \mathbb{R} \rightarrow E^{\theta}$, что $\phi(0)=u^{0}$ и для любого $s \in \mathbb{R}$ равенство $S(t) \phi(s)=\phi(t+s)$ имеет место при $t \geqslant 0$.

Поскольку множество значений полугруппы $S(t)$ не совпадает со всем пространством $E^{\theta}$, факт существования отрицательной или полной орбиты налагает определенные ограничения на $u^{0}$. Кроме того, поскольку отображение $S(t)$, вообще говоря, не является биективным, отрицательная орбита - если она существует - не обязательно единственна. Пусть отрицательная орбита, проходящая через $u^{0}$, определена как объединение всех отрицательных орбит, проходящих через $u^{0}$. Тогда

$$
\gamma^{-}\left(u^{0}\right)=\bigcup_{t \geqslant 0} H\left(t, u^{0}\right)
$$

где $H\left(t, u^{0}\right)=\left\{y \in E^{\theta}:\right.$ найдется отрицательная орбита, проходящая через $u^{0}$ и определенная отображением $\left.\phi:(-\infty, 0] \rightarrow E^{\theta}, \phi(0)=u^{0}, \phi(-t)=y\right\}$.

Полная орбита $\gamma\left(u^{0}\right)$, проходящая через $u^{0}$, определена как $\gamma\left(u^{0}\right)=\gamma^{-}\left(u^{0}\right) \cup \gamma^{+}\left(u^{0}\right)$. Будем говорить, что множество $B \subset E^{\theta}$ притягивает множество $C$ посредством $S(t)$, если $\operatorname{dist}(S(t) C, B) \rightarrow 0$ в норме пространства $E^{\theta}$ при $t \rightarrow \infty$. Будем говорить, что множество $Q \subset E^{\theta}$ инвариантно относительно $S(\cdot)$, если для любого $u^{0} \in Q$ найдется такая полная орбита $\gamma\left(u^{0}\right)$, проходящая через $u^{0}$, что $\gamma\left(u^{0}\right) \subset Q$ или, эквивалентно, $S(t) Q=Q$ для любого $t \geqslant 0$. Множество $\mathcal{A} \subseteq E^{\theta}$ называется глобальным аттрактором для полугруппы $\{S(t): t \geqslant 0\}$, если оно компактно, инвариантно и притягивает любые ограниченные подмножества пространства $E^{\theta}$.

\section{1. АТТРАКТОРЫ АБСТРАКТНЫХ ПАРАБОЛИЧЕСКИХ ЗАДАЧ}

Предположим, что оператор $A$ порождает (см. (1.1.3)) аналитическую $C_{0}$-полугруппу, удовлетворяющую оценке

$$
\left\|e^{t A}\right\| \leqslant M e^{-t \omega}, \quad t \geqslant 0,
$$

где $\omega>0$, резольвента $(\lambda I-A)^{-1}$ компактна для некоторого $\lambda \in \rho(A)$, a $f(\cdot): E^{\theta} \rightarrow E-$ глобально липшицева, ограниченная (т.е. найдется такая константа $K>0$, что $\|f(x)\|_{E} \leqslant K$ для всех $\left.x \in E^{\theta}\right)$ и непрерывно дифференцируемая по Фреше функция.

3.1.1. Инвариантные и компактные множества. Покажем, что условия, наложенные на $A$ и $f(\cdot)$ в задаче $(1.2 .16)$, достаточны для существования у полугруппы $\{S(t): t \geqslant 0\}$ глобального аттрактора. Напомним, что для любого множества $B \subset E^{\theta}$ множества

$$
\gamma^{+}(B)=\bigcup_{u^{0} \in B} \gamma^{+}\left(u^{0}\right), \quad \gamma^{-}(B)=\bigcup_{u^{0} \in B} \gamma^{-}\left(u^{0}\right), \quad \gamma(B)=\cup_{u^{0} \in B} \gamma\left(u^{0}\right)
$$

являются, соответственно, положительной, отрицательной и полной орбитами, проходящими через $B$ (если они существуют).

Для любого множества $B \subset E^{\theta}$ определим $\omega$-предельное множество $\omega(B)$ и $\alpha$-предельное множество $\alpha(B)$ следующим образом:

$$
\omega(B)=\bigcap_{s \geqslant 0} \overline{\bigcup_{t \geqslant s} S(t) B}, \quad \alpha(B)=\bigcap_{s \geqslant 0} \overline{\bigcup_{t \geqslant s} H(t, B)} .
$$

Напомним следующее важное утверждение (см. [89]). 
Лемма 3.1.1. Если множество $S \subset E^{\theta}$ инвариантно относительно $\{S(t): t \geqslant 0\} u v \in S$, то найдется полная орбита, проходящая через $v$.

Лемма 3.1.2. Точка $v$ лежит в $\omega(B)$ тогда и только тогда, когда существуют такие последовательности $t_{n} \rightarrow+\infty u v_{n} \in B$, что $S\left(t_{n}\right) v_{n} \rightarrow v$.

Лемма 3.1.3. Точка $v$ лежит в $\alpha(B)$ тогда и только тогда, когда существуют такие последовательности $t_{n} \rightarrow+\infty$ u $v_{n} \in B$, что отрицательная орбита $\phi_{n}:(-\infty, 0] \rightarrow E^{\theta}$, удовлетворяющая условиям $\phi_{n}(0)=v_{n} u \phi_{n}\left(-t_{n}\right) v_{n} \rightarrow v$, проходит через каждую точку $v_{n}$.

Лемма 3.1.4. Для любого ограниченного множества $B \subset E^{\theta}$ множество $\gamma^{+}(B)$ ограничено u $S(t) \gamma^{+}(B)$ предкомпактно для любого $t>0$.

Доказательство. Известно, что отображение $S(t): E^{\theta} \rightarrow E^{\theta}$ компактно. Используя формулу вариации постоянной, стандартные оценки и предположения, сделанные относительно $A$ и $f(\cdot)$, заключаем, что орбита $\gamma^{+}(B)$ ограничена в $E^{\theta}$, если $B$ ограничено в $E^{\theta}$. Поскольку $\gamma^{+}(B)$ ограничена, имеем тогда, что $\{S(s) B: s \geqslant t\}=S(t) \gamma^{+}(B)$ компактно.

Лемма 3.1.5. Для любого $u^{0} \in E^{\theta}$ множество $\omega\left(u^{0}\right)$ непусто, связно, компактно, инвариантно и притягивает $u^{0}$.

Доказательство. Легкл видеть, что из компактности множества $\overline{\gamma^{+}\left(u^{0}\right)}$ следует, что $\omega\left(u^{0}\right)$ непусто и $\omega\left(u^{0}\right)$ ограничено. Кроме того, из непрерывности отображения $t \mapsto S(t) u^{0}$ и определения $\omega\left(u^{0}\right)$ следует, что $\omega\left(u^{0}\right)$ связно. Докажем, что $\omega\left(u^{0}\right)$ инвариантно. Действительно, если $v \in \omega\left(u^{0}\right)$, то найдется такая последовательность $t_{n} \rightarrow+\infty$, что $S\left(t_{n}\right) u^{0} \rightarrow v$. Из непрерывности $S(t): E^{\theta} \rightarrow E^{\theta}$ получаем, что $S(t) S\left(t_{n}\right) u^{0} \rightarrow S(t) v$ и, следовательно, $S(t) v \in \omega\left(u^{0}\right)$. С другой стороны, если $w \in \omega\left(u^{0}\right)$, то найдется такая последовательность $t_{n} \rightarrow \infty$ (без ограничения общности можно считать, что $t_{n} \geqslant t, n \in \mathbb{N}$ ), что $S\left(t_{n}\right) u^{0} \rightarrow w$. Поскольку

$$
\left\{S\left(t_{n}-t\right) u^{0}: n \in \mathbb{N}\right\}=\left\{S(t) S\left(t_{n}-2 t\right) u^{0}: n \in \mathbb{N}\right\}
$$

относительно компактно, найдется такая точка $z \in E^{\theta}$, что $S\left(t_{n}-t\right) u^{0} \rightarrow z$ и $z \in \omega\left(u^{0}\right)$. Из единственности предела следует, что $S(t) z=w$. Теперь компактность легко вытекает из инвариантности, компактности $S(t)$ и ограниченности $\omega\left(u^{0}\right)$. Осталось показать, что $\omega\left(u^{0}\right)$ притягивает $u^{0}$ посредством $\{S(t): t \geqslant 0\}$. Предположим, что существуют такие $\epsilon>0$ и последовательность $t_{n} \rightarrow \infty$, что $\operatorname{dist}\left(S\left(t_{n}\right) u^{0}, \omega\left(u^{0}\right)\right) \geqslant \epsilon$. Поскольку множество $\gamma^{+}\left(u^{0}\right)$ компактно, найдутся такие подпоследовательность последовательности $\left\{t_{n}\right\}$ (сохраним для нее обозначение $\left\{t_{n}\right\}$ ) и $y \in E^{\theta}$, что $S\left(t_{n}\right) u^{0} \rightarrow y$. Отсюда следует, что $y \in \omega\left(u^{0}\right)$ и, следовательно,

$$
\lim _{t_{n} \rightarrow \infty} \operatorname{dist}\left(S\left(t_{n}\right) u^{0}, \omega\left(u^{0}\right)\right)=0,
$$

что противоречит сделанному предположению и доказывает, что $\omega\left(u^{0}\right)$ притягивает $u^{0}$ посредством $\{S(t): t \geqslant 0\}$.

Лемма 3.1.6. Предположим, что $u^{0} \in E^{\theta}$ такова, что найдется отрицательная орбита $\phi:(-\infty, 0] \rightarrow E^{\theta}$, проходящая через $u^{0}$ и замыкание $\overline{\phi((-\infty, 0])}$ компактно. Пусть

$$
\alpha_{\phi}\left(u^{0}\right)=\left\{v \in E^{\theta} \mid \exists t_{n} \rightarrow \infty: \phi\left(-t_{n}\right) \rightarrow v\right\} .
$$

Тогда множество $\alpha_{\phi}\left(u^{0}\right)$ непусто, связно, компактно и инвариантно.

Доказательство. Легко видеть, что

$$
\alpha_{\phi}\left(u^{0}\right)=\bigcap_{t \geqslant 0} \overline{\phi((-\infty, t])}
$$

отсюда следует, что $\alpha_{\phi}\left(u^{0}\right)$ непусто, компактно и связно. Осталось доказать, что $\alpha_{\phi}\left(u^{0}\right)$ инвариантно. Действительно, если $v \in \alpha_{\phi}\left(u^{0}\right)$, то найдется такая последовательность $t_{n} \rightarrow+\infty$, что $\phi\left(-t_{n}\right) \rightarrow v$. Из непрерывности отображения $S(t): E^{\theta} \rightarrow E^{\theta}$ получим, что $S(t) \phi\left(-t_{n}\right)=\phi\left(t-t_{n}\right) \rightarrow S(t) v$ и, следовательно, $S(t) v \in \alpha_{\phi}\left(u^{0}\right)$. С другой стороны, если 
$w \in \alpha_{\phi}\left(u^{0}\right)$, то найдется такая последовательность $t_{n} \rightarrow \infty$ (без ограничения общности можем предположить, что $\left.t_{n} \geqslant t, n \in \mathbb{N}\right)$, что $\phi\left(-t_{n}\right) \rightarrow w$. Поскольку множество $\left\{\phi\left(-t_{n}-t\right): n \in \mathbb{N}\right\}$ относительно компактно, переходя при необходимости к подпоследовательности, видим, что найдется такая точка $z \in E^{\theta}$, что $\phi\left(-t_{n}-t\right) \rightarrow z$ и $z \in \alpha_{\phi}\left(u^{0}\right)$. Из единственности предела следует, что $S(t) z=w$.

Лемма 3.1.7. Орбита любого ограниченного множества $B \subset E^{\theta}$ ограничена. Для каждого ограниченного множества $B \subset E^{\theta}$ найдутся такие момент $\tau_{B}$ и константа $N$ (не зависящая om $B)$, чmo

$$
\sup _{t \geqslant \tau_{B}} \sup _{w \in S(t) B}\|w\|_{E^{\theta}} \leqslant N
$$

Кроме того,

$$
\sup _{\substack{B \subset E^{\theta}, B \text { ограничено }}} \sup _{v \in \omega(B)}\|v\|_{E^{\theta}} \leqslant N
$$

Доказательство. Первое утверждение тривиально следует из формулы вариации постоянной, а (3.1.2) следует из (3.1.1) и определения $\omega(B)$. Осталось доказать (3.1.1). Для данного ограниченного множества $B \subset E^{\theta}$ и $v \in B$ из (2.3.1) получаем, что

$$
S(t) v=e^{t A} v+\int_{0}^{t} e^{(t-s) A} f(S(s) v) d s, \quad t \geqslant 0 .
$$

Используя наложенные на $f(\cdot)$ и $A$ условия, заключаем, что найдется такая константа $K$, что $\|f(w)\|_{E} \leqslant K$ для всех $w \in E^{\theta}$ и

$$
\|S(t) v\|_{E^{\theta}} \leqslant M e^{-\beta t}\|v\|_{E^{\theta}}+M K \int_{0}^{t}(t-s)^{-\theta} e^{-\beta(t-s)} d s .
$$

Из того факта, что $v$ лежит в ограниченном подмножестве пространства $E^{\theta}$, следует, что существует такой момент времени $\tau_{B}$, что

$$
\|S(t) v\|_{E^{\theta}} \leqslant 1+M K \int_{0}^{\infty} \xi^{-\theta} e^{-\beta \xi} d \xi=: N, \quad t \geqslant \tau_{B} .
$$

Поскольку $N$ не зависит от $B \subset E^{\alpha}$, получаем требуемый результат.

Лемма 3.1.8. Если $B \subset E^{\theta}$-ограниченное множество, то $\omega(B)$ непусто, компактно, инвариантно и притягивает $B$ посредством $\{S(t): t \geqslant 0\}$. Кроме того, если $B$ связно, то и $\omega(B)$ тоже связно.

Доказательство. Из леммы 3.1.7 следует, что $\gamma^{+}(B)$ ограничено; таким образом, $S(t) \gamma^{+}(B)$ компактно. Из того факта, что $\omega\left(u^{0}\right) \subset \omega(B)$ для всех $u^{0} \in B$, следует, что $\omega(B)$ непусто. Чтобы доказать, что $\omega(B)$ компактно, заметим, что $\overline{S(t) \gamma^{+}(B)}$ компактно и содержит $\omega(B)$. Теперь покажем, что $\omega(B)$ инвариантно. Если $u \in \omega(B)$, то найдутся такие последовательности $t_{n} \rightarrow \infty$ и $v_{n} \in B$, что $S\left(t_{n}\right) v_{n} \rightarrow u$. Отсюда следует, что $S\left(t+t_{n}\right) v_{n} \rightarrow S(t) v$ и $S(t) v \in \omega(B)$. Следовательно, $S(t) \omega(B) \subset \omega(B)$. С другой стороны, если $v \in \omega(B)$, то найдутся такие последовательности $t_{n} \rightarrow \infty$ и $v_{n} \in B$, что $S\left(t_{n}\right) v_{n} \rightarrow v$. Поскольку $\left\{S\left(t_{n}-t\right) v_{n}\right\}$ компактно, найдутся такие подпоследовательность (обозначим ее снова $\left\{S\left(t_{n}-t\right) v_{n}\right\}$ ) и точка $u \in E^{\theta}$, что $S\left(t_{n}-t\right) v_{n} \rightarrow u$. Следовательно, $u \in \omega(B), S(t) u=v$ и $v \in S(t) \omega(B)$. Таким образом, $\omega(B) \subset S(t) \omega(B)$, и мы получаем требуемое.

Далее, покажем, что $\omega(B)$ притягивает $B$. Предположим, что для некоторого $\epsilon>0$ найдутся такие последовательности $t_{n} \rightarrow \infty$ и $u_{n} \in B$, что $\operatorname{dist}\left(S\left(t_{n}\right) u_{n}, \omega(B)\right) \geqslant \epsilon$. Из компактности 
$\left\{S\left(t_{n}\right) u_{n}\right\}$ следует, что существуют такие точка $w \in E^{\theta}$ и подпоследовательность (которую обозначим снова $\left.\left\{S\left(t_{n}\right) u_{n}\right\}\right)$, что $S\left(t_{n}\right) u_{n} \rightarrow w$. Тогда $w \in \omega(B)$, противоречние. Для доказательства связности $\omega(B)$ нужно воспользоваться непрерывностью отображения $(t, u) \mapsto S(t) u$.

Теорема 3.1.1. Если $B_{N}=\left\{u \in E^{\theta}:\|u\|_{E^{\theta}} \leqslant N\right\}$, mо $\omega\left(B_{N}\right)$ - глобалъный аттрактор для $\{S(t): t \geqslant 0\}$.

Доказательство. Ясно, что $\omega\left(B_{N}\right)$ компактно, инвариантно, связно и притягивает $B_{N}$. Вопервых, заметим, что для любого ограниченного множества $B$ найдется такой момент $\tau_{B}$, что $S(t) B \subset B_{N}$ для всех $t \geqslant \tau_{B}$. Поскольку $B_{N}$ притягивается множеством $\omega\left(B_{N}\right)$ и $B_{N}$ притягивает $B$, получим требуемый результат.

Лемма 3.1.9. Пусть ограниченное множество $B \subset E^{\theta}$ таково, что множество $\overline{\gamma^{-}(B)}$ непусто и компактно. Тогда $\alpha(B)$ непусто, компактно и инвариантно.

Доказательство. Если $u^{0} \in B$ таково, что существует отрицательная орбита $\phi:(-\infty, 0] \rightarrow E^{\theta}$, проходящая через $u^{0}$, то замыкание $\overline{\{\phi(t): t \in(-\infty, 0]\}}$ компактно и найдутся такие последовательност $t_{n} \rightarrow \infty$ и точка $v \in E^{\theta}$, что $\phi\left(-t_{n}\right) \rightarrow v$. Из леммы 3.1 .3 следует, что $v \in \alpha(B)$ и $\alpha(B) \neq \varnothing$. Из определения и сделанных предположений следует, что $\alpha(B)$ компактно.

Для доказательства инвариантности $\alpha(B)$ заметим, что если $v \in \alpha(B)$, то найдутся такие последовательности $t_{n} \rightarrow \infty$ и $v_{n} \in B$ и соответствующие орбиты $\phi_{n}:(-\infty, 0] \rightarrow E^{\theta}$, проходящие через $v_{n}\left(\phi_{n}(0)=v_{n}\right)$, что $\phi_{n}\left(-t_{n}\right) \rightarrow v$. В силу непрерывности $S(t), t \geqslant 0$, заключаем, что $\phi_{n}\left(t-t_{n}\right)=S(t) \phi_{n}\left(-t_{n}\right) \rightarrow S(t) v$ и $S(t) v \in \alpha(B)$. Таким образом, $S(t) \alpha(B) \subset \alpha(B)$. С другой стороны, если $v \in \alpha(B)$, то найдутся такие последовательности $t_{n} \rightarrow \infty$ и $v_{n} \in B$ и соответствующие отрицательные орбиты $\phi_{n}:(-\infty, 0] \rightarrow E^{\theta}$, что $\phi_{n}\left(-t_{n}\right) \rightarrow v$. Поскольку $\left\{\phi_{n}\left(-t-t_{n}\right)\right\}$ лежит в компактном множестве, найдутся такие точка $z \in E^{\theta}$ и подпоследовательность (которую мы также обозначим через $\left.\left\{\phi_{n}\left(-t-t_{n}\right)\right\}\right)$, что $\phi_{n}\left(-t-t_{n}\right) \rightarrow z$. Следовательно, $z \in \alpha(B)$ и $S(t) z=v$. Итак, $\alpha(B) \subset S(t) \alpha(B)$, и инвариантность доказана.

3.1.2. Точки равновесия. Опишем структуру аттракторов задачи (1.2.16). Начнем с простейших элементов аттракторов, а именно, равновесных решений. Равновесные решения задачи (1.2.16) - это решения, не зависящие от времени: $u(t)=u, t \geqslant 0$; они удовлетворяют уравнению

$$
A u+f(u)=0 .
$$

Будем называть решение $u^{*}$ уравнения (3.1.4), т.е. точку равновесия $u^{*}$, гиперболической, если спектр $\sigma\left(A+f^{\prime}\left(u^{*}\right)\right)$ не пересекается с мнимой осью, т.е. $\sigma\left(A+f^{\prime}\left(u^{*}\right)\right) \cap i \mathbb{R}=\varnothing$.

Предложение 3.1.1. Предположим, что все точки равновесия задачи (1.2.16) гиперболические. Тогда существует лищь конечное их число, и это число нечетно.

Доказательство. Во-первых, заметим, что, поскольку функция $f(\cdot): E^{\theta} \rightarrow E$ ограничена, все решения уравнения (3.1.4) удовлетворяют уравнению

$$
I u+A^{-1} f(u)=0
$$

и, таким образом, $\|u\|_{E^{1}} \leqslant\|f(u)\|_{E} \leqslant C_{1}$. Если мы рассмотрим шар радиуса, большего $\left\|A^{-1}\right\| K$, то оператор $A^{-1} f(\cdot)$ отобразит шар $\mathcal{U}_{E^{\theta}}\left(0,\left\|A^{-1}\right\| K\right) \subset E^{\theta}$ в себя. По теореме Шаудера о неподвижной точке (см. [21, теорема 21.5]) имеем

$$
\gamma\left(I+A^{-1} f(\cdot), \partial \mathcal{U}_{E^{\theta}}\left(0,\left\|A^{-1}\right\| K\right)\right)=1,
$$

т.е. существует по крайней мере одна такая неподвижная точка уравнения $I u^{*}+A^{-1} f\left(u^{*}\right)=0$, что $u^{*} \in \mathcal{U}_{E^{\theta}}\left(0,\left\|A^{-1}\right\| K\right)$. Поскольку оператор $A^{-1} f(\cdot): E^{\theta} \rightarrow E^{\theta}$ компактен, заключаем, что множество $\mathcal{E}=\{u: A u+f(u)=0\}$ компактно в $E^{\theta}$. Кроме того, любая неподвижная точка $u^{*}$ изолирована и $\left|\operatorname{ind}\left(u^{*}, I+A^{-1} f(\cdot)\right)\right|=1$, поскольку $u^{*}$ гиперболическая, и $\mathcal{N}\left(I+A^{-1} f^{\prime}\left(u^{*}\right)\right)=\{0\}$ (см. [21, теорема 21.6]). Если число неподвижных точек бесконечно, т.е. имеем последовательность 
$\left\{u_{i}^{*}\right\}_{i=1}^{\infty}$, то последовательность $-A^{-1} f\left(u_{i}^{*}\right)=u_{i}^{*} \rightarrow u_{\infty}^{*}$ сходится на некоторой подпоследовательности $i \in \mathbb{N}^{\prime} \subseteq \mathbb{N}$, что противоречит факту изолированности неподвижной точки $u_{\infty}^{*}$. Таким образом, число точек равновесия конечно. Согласно [21, теорема 20.6] имеем

$$
1=\gamma\left(I+A^{-1} f(\cdot), \partial \mathcal{U}_{E^{\theta}}\left(0,\left\|A^{-1}\right\| K\right)\right)=\sum_{i=1}^{d} \operatorname{ind}\left(u_{i}^{*}, I+A^{-1} f(\cdot)\right)
$$

и, следовательно, $d=2 k+1$ для некоторого целого $k \geqslant 0$.

3.1.3. Неустойчивые многообразия. Далее мы покажем, что помимо стационарных решений аттракторы содержат множество других решений. Легко видеть, что каждое обобщенное решение задачи (1.2.16) остается ограниченным при любом $u^{0} \in E^{\theta}$, т.е.

$$
\sup _{t \geqslant 0}\left\|u\left(t, u^{0}\right)\right\|_{E^{\theta}}<\infty .
$$

Если мы покажем, что обобщенное решение $u\left(\cdot, u^{0}\right)$, определенное на $\mathbb{R}$, принимает значения в $E^{\theta}$ и является ограниченным, то оно должно принадлежать аттрактору $\mathcal{A}$. Таким образом, чтобы получить решение, лежащее в аттракторе, достаточно показать, что решение определено и ограничено в $\overline{\mathbb{R}}_{-}=(-\infty, 0]$. Введем неустойчивое множество

$$
W^{u}\left(u^{*}\right)=\left\{\eta \in E^{\theta}: u(t, \eta) \text { определено для всех } t \leqslant 0 \text { и } u(t, \eta) \rightarrow u^{*} \text { при } t \rightarrow-\infty\right\} .
$$

Прежде чем строить $W^{u}\left(u^{*}\right)$ (по крайней мере в окрестности точки $u^{*}$ ), кратко обсудим линейные задачи. Мы естественным образом придем к линейному случаю, если выполним замену переменных $v(\cdot)=u(\cdot)-u^{*}$ в задаче (1.2.16), добавим и затем вычтем $f^{\prime}\left(u^{*}\right) v(\cdot)$ в правой части. Получим

$$
\begin{aligned}
& v^{\prime}(t)=\left(A+f^{\prime}\left(u^{*}\right)\right) v(t)+f\left(v(t)+u^{*}\right)-f\left(u^{*}\right)-f^{\prime}\left(u^{*}\right) v(t), \\
& v(0)=u^{0}-u^{*}=v^{0} .
\end{aligned}
$$

В этом уравнении для весьма малого $v^{0}$ слагаемое $f\left(v(t)+u^{*}\right)-f\left(u^{*}\right)-f^{\prime}\left(u^{*}\right) v(t)$ также является малым. Естественно выяснить, что произойдет, если пренебречь нелинейностью, т.е. исследовать задачу Коши

$$
\begin{aligned}
& v^{\prime}(t)=\left(A+f^{\prime}\left(u^{*}\right)\right) v(t), \\
& v(0)=v^{0} .
\end{aligned}
$$

Оператор $f^{\prime}\left(u^{*}\right)$ лежит в $B\left(E^{\theta}, E\right)$; следовательно, оператор $A_{u^{*}}=A+f^{\prime}\left(u^{*}\right)$, определенный на $D(A)$, является генератором аналитической $C_{0}$-полугруппы с компактной резольвентой (см. [26]). Известно, что спектр этого генератора состоит лишь из изолированных собственных значений с конечномерными корневыми подпространствами (так называемый дискретный спектр). Часть $\sigma^{+}$спектра оператора $A+f^{\prime}\left(u^{*}\right)$ лежит правее мнимой оси и состоит из конечного числа собственных значений конечной кратности (см. рис. 1).

Рассмотрим разложение $E^{\theta}=P\left(\sigma^{+}\right) E^{\theta} \oplus\left(I-P\left(\sigma^{+}\right)\right) E^{\theta}$, используя проектор

$$
P\left(\sigma^{+}\right):=P\left(\sigma^{+}, A_{u^{*}}\right):=\frac{1}{2 \pi i} \int_{\partial U\left(\sigma^{+}\right)}\left(\zeta I-A_{u^{*}}\right)^{-1} d \zeta
$$

определенный множеством $\sigma^{+}$, где $U\left(\sigma^{+}\right)$- такая окрестность $\sigma^{+}$, что $U\left(\sigma^{+}\right) \cap i \mathbb{R}=\varnothing$. Видим, что существует такое $\omega>0$, что

$$
\begin{cases}\left\|e^{t\left(A+f^{\prime}\left(u^{*}\right)\right)} P\left(\sigma^{+}\right)\right\|_{B\left(E^{\theta}\right)} \leqslant M e^{\omega t}, & t \leqslant 0, \\ \left\|e^{t\left(A+f^{\prime}\left(u^{*}\right)\right)}\left(I-P\left(\sigma^{+}\right)\right)\right\|_{B\left(E^{\theta}, E^{\phi}\right)} \leqslant M t^{\theta-\phi} e^{-\omega t}, & \phi>\theta, \quad t \geqslant 0 .\end{cases}
$$

Если $P\left(\sigma^{+}\right)$- спектральный проектор, определенный частью спектра оператора $\left(A+f^{\prime}\left(u^{*}\right)\right)$, лежащей правее мнимой оси, и $v_{0} \in P\left(\sigma^{+}\right) E^{\theta}$, то решение $v\left(t, v_{0}\right)$ уравнения (3.1.7) существует для отрицательных $t$, причем $v\left(t, v_{0}\right) \rightarrow 0$ при $t \rightarrow-\infty$ и $v(t)+u^{*} \rightarrow u^{*}$ при $t \rightarrow-\infty$. 


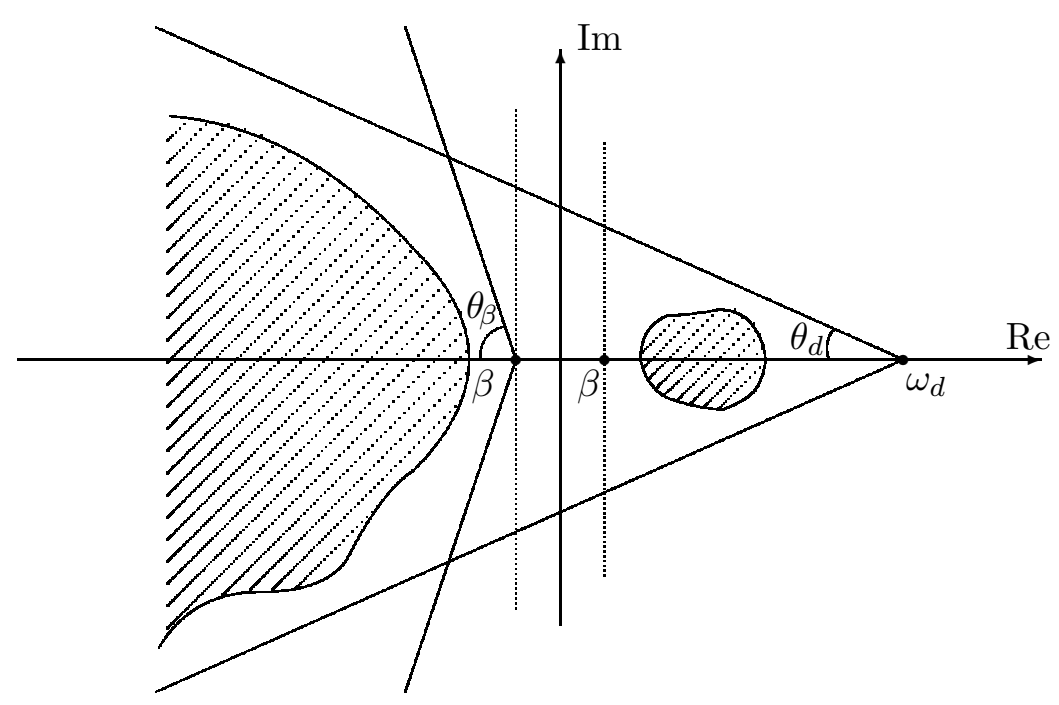

Рис. 1

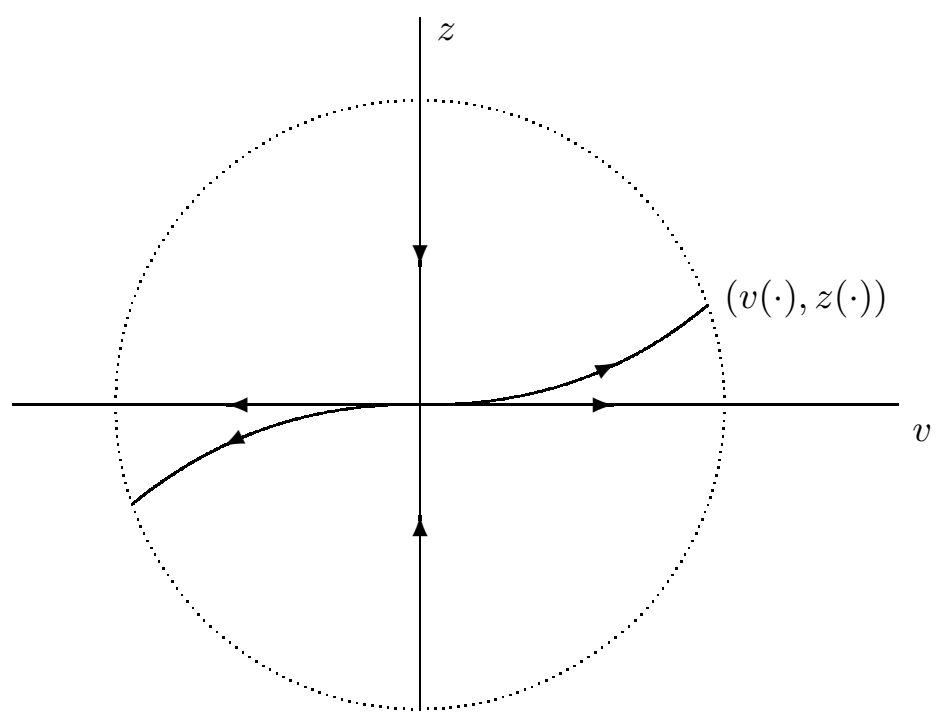

Рис. 2

Если произвести возмущение (3.1.7) малой нелинейностью, обнаружим, решения уравнения (3.1.6), существующие для всех отрицательных $t$. Разумеется, начальные данные, при которых такие решения существуют, лежат теперь не в $P\left(\sigma^{+}\right) E^{\theta}$, а в нелинейном многообразии вблизи него (см. рис. 2).

Теорема 3.1.2 (см. [89]). Предположим, что и* - гиперболическая равновесная точка задачи (1.2.16), являющаяся изолированным решением уравнения (3.1.4). Тогда существует такал окрестность $U$ точки $u^{*}$ в $E^{\theta}$, что локальное неустойчивое многообразие $W^{u}\left(u^{*}\right)$ задается следующим образом:

$$
W_{\delta}^{u}\left(u^{*}\right)=\left\{w(\cdot) \in W^{u}\left(u^{*}\right):\left\|w(\cdot)-u^{*}\right\|_{E^{\theta}}<\delta\right\}=\left\{w(\cdot):(v(\cdot), z(\cdot)) \in E^{\theta}: u \in U\right\} .
$$


Доказательство. Переписав задачу $(1.2 .16)$ для $w(t)=u(t)-u^{*}$, чтобы иметь возможность работать в окрестности точки $u^{*} \in \mathcal{E}$, приходим к уравнению

$$
w^{\prime}(t)=\left(A+f^{\prime}\left(u^{*}\right)\right) w(t)+f\left(w(t)+u^{*}\right)-f\left(u^{*}\right)-f^{\prime}\left(u^{*}\right) w(t) .
$$

Если $w(\cdot)$ - обобщенное решение задачи (3.1.10), то можем записать

$$
v(t)=P\left(\sigma^{+}\right) w(t), \quad z(t)=\left(I-P\left(\sigma^{+}\right)\right) w(t) .
$$

Таким образом, если обозначить через $B$ сужение оператора $\left(A+f^{\prime}\left(u^{*}\right)\right)$ на $P\left(\sigma^{+}\right) E^{\theta}$ и через $\tilde{A}$ сужение оператора $\left(A+f^{\prime}\left(u^{*}\right)\right)$ на $\left(I-P\left(\sigma^{+}\right)\right) E^{\theta}$, то получим

$$
v^{\prime}(t)=B v(t)+P\left(\sigma^{+}\right) f\left(v(t)+z(t)+u^{*}\right)-P\left(\sigma^{+}\right) f\left(u^{*}\right)-P\left(\sigma^{+}\right) f^{\prime}\left(u^{*}\right)(v(t)+z(t))
$$

и

$z^{\prime}(t)=\tilde{A} z(t)+\left(I-P\left(\sigma^{+}\right)\right) f\left(v(t)+z(t)+u^{*}\right)-\left(I-P\left(\sigma^{+}\right)\right) f\left(u^{*}\right)-\left(I-P\left(\sigma^{+}\right)\right) f^{\prime}\left(u^{*}\right)(v(t)+z(t))$

(см. рис. 1 , на котором изображено положение частей спектра). Запишем

$$
\begin{gathered}
H(v, z)=P\left(\sigma^{+}\right) f\left(v(t)+z(t)+u^{*}\right)-P\left(\sigma^{+}\right) f\left(u^{*}\right)-P\left(\sigma^{+}\right) f^{\prime}\left(u^{*}\right)(v(t)+z(t)), \\
G(v, z)=\left(I-P\left(\sigma^{+}\right)\right) f\left(v(t)+z(t)+u^{*}\right)-\left(I-P\left(\sigma^{+}\right)\right) f\left(u^{*}\right)-\left(I-P\left(\sigma^{+}\right)\right) f^{\prime}\left(u^{*}\right)(v(t)+z(t)) .
\end{gathered}
$$

Таким образом, в начале координат $(0,0)$ функции $H$ и $G$ и их производные обращаются в нуль. В силу непрерывной дифференцируемости функций $H$ и $G$ получаем следующие неравенства для заданных $\rho>0$ и $\delta>0$, удовлетворяющих условию $\|v\|_{E^{\theta}}+\|z\|_{E^{\theta}}<\delta$ :

$$
\left\{\begin{array}{l}
\|H(v, z)\|_{E} \leqslant \rho, \\
\|G(v, z)\|_{E} \leqslant \rho, \\
\|H(v, z)-H(\tilde{v}, \tilde{z})\|_{E} \leqslant \rho\left(\|v-\tilde{v}\|_{E^{\theta}}+\|z-\tilde{z}\|_{E^{\theta}}\right), \\
\|G(v, z)-G(\tilde{v}, \tilde{z})\|_{E} \leqslant \rho\left(\|v-\tilde{v}\|_{E^{\theta}}+\|z-\tilde{z}\|_{E^{\theta}}\right) .
\end{array}\right.
$$

Используя введенные выше обозначения, перепишем уравнение (3.1.10) в следующем виде:

$$
\left\{\begin{array}{l}
v^{\prime}(t)=B v(t)+H(v(t), z(t)), \\
z^{\prime}(t)=\tilde{A} z(t)+G(v(t), z(t)),
\end{array}\right.
$$

где $H$ и $G$ удовлетворяют (3.1.11) для всех $v(\cdot) \in P\left(\sigma^{+}\right) E^{\theta}$ и $z(\cdot) \in\left(I-P\left(\sigma^{+}\right)\right) E^{\theta}$. Кроме того, для некоторых положительных $M$ и $\omega$, в силу аналитичности $C_{0}$-полугруппы и разложения пространства $E^{\theta}=P\left(\sigma^{+}\right) E^{\theta} \oplus\left(I-P\left(\sigma^{+}\right)\right) E^{\theta}$, имеем

$$
\begin{cases}\left\|e^{\tilde{A} t} z\right\|_{E^{\theta}} \leqslant M e^{-\omega t}\|z\|_{E^{\theta}}, & t \geqslant 0 \\ \left\|e^{\tilde{A} t} z\right\|_{E^{\theta}} \leqslant M t^{-\theta} e^{-\omega t}\|z\|_{E}, & t \geqslant 0 \\ \left\|e^{B t} v\right\|_{E^{\theta}} \leqslant M e^{\omega t}\|v\|_{E}, & t \leqslant 0\end{cases}
$$

Заметим, что

$$
\left\|e^{\tilde{A} t} z\right\|_{E^{\theta}}=\left\|(-A)^{\theta} e^{\tilde{A} t} z\right\|=\left\|(-A)^{\theta} \tilde{A}^{-\theta}(-\tilde{A})^{\theta} e^{\tilde{A} t} z\right\|
$$

поскольку $(-A)^{\theta} \tilde{A}^{-\theta}$ ограничено, получим неравенства (3.1.13). Заметим также, что $P\left(\sigma^{+}\right) E^{\theta}$ и $P\left(\sigma^{+}\right) E$ - те же самые конечномерные подпространства пространства $E$, и в этом смысле $E^{\theta}$ норма эквивалентна $E$-норме в нем, т.е.

$$
\left\|P\left(\sigma^{+}\right) A P\left(\sigma^{+}\right)\right\|_{B(E)}=\left\|\frac{1}{2 \pi i} \int_{\Gamma} A(\lambda I-A)^{-1} d \lambda\right\| \leqslant \text { const . }
$$

Покажем теперь, что при некотором малом $\rho>0$ существует локальное неустойчивое многообразие для точки $u^{*} \in \mathcal{E}$ :

$$
W^{u}=\left\{(v(\cdot), z(\cdot)): z(\cdot) \in\left(I-P\left(\sigma^{+}\right)\right) E^{\theta}, v(\cdot) \in P\left(\sigma^{+}\right) E^{\theta}\right\} .
$$


Решение $(v(t), z(t))$ должно стремиться к нулю при $t \rightarrow-\infty$ и, в частности, должно быть ограниченным. Поскольку

$$
z(t)=e^{\tilde{A}\left(t-t_{0}\right)} z\left(t_{0}\right)+\int_{t_{0}}^{t} e^{\tilde{A}(t-s)} G(v(s), z(s)) d s,
$$

устремляя $t_{0} \mathrm{~K}-\infty$, имеем

$$
z(t)=\int_{-\infty}^{t} e^{\tilde{A}(t-s)} G(v(s), z(s)) d s
$$

Обобщенное решение задачи (3.1.12) является векторнозначной функцией $\zeta(t)=\left(\begin{array}{l}v(t) \\ z(t)\end{array}\right)$, удовлетворяющей уравнению

$$
\zeta(t)=\Theta(\eta, \zeta(t)) \equiv\left(\begin{array}{c}
e^{B(t-\tau)} \eta+\int_{\tau}^{t} e^{B(t-s)} H(v(s), z(s)) d s \\
\int_{-\infty}^{t} e^{\tilde{A}(t-s)} G(v(s), z(s)) d s
\end{array}\right) .
$$

Теперь рассмотрим уравнение (3.1.14) в пространстве

$$
\Upsilon=C_{0}\left((-\infty, \tau] ; P E^{\theta}\right) \times C_{0}\left((-\infty, \tau] ;(I-P) E^{\theta}\right),
$$

снабженном sup-нормой, при условии $v(-\infty)=z(-\infty)=0$. Покажем, что $\Theta(\eta, \cdot): \Upsilon \rightarrow \Upsilon$.

Возьмем $\zeta(\cdot) \in \Upsilon$; согласно (3.1.11), для любого $\epsilon>0$ существует такое число $-\infty<t_{\epsilon}<0$, что

$$
\|G(\zeta(s))\|_{(I-P) E} \leqslant \epsilon, \quad\|H(\zeta(s))\|_{P E} \leqslant \epsilon, \quad s \leqslant t_{\epsilon} .
$$

Из (3.1.13) следует, что $e^{B(\cdot-\tau)} \eta \in C_{0}\left((-\infty, \tau] ; P E^{\theta}\right)$ для любого $\eta \in P E^{\theta}$ и

$$
\begin{gathered}
\left\|(-A)^{\theta} \int_{\tau}^{t} e^{(t-s) B} H(\zeta(s)) d s\right\| \leqslant C\left\|\int_{\tau}^{t} e^{(t-s) B} H(\zeta(s)) d s\right\| \leqslant \\
\leqslant C\left\|e^{\left(t-t_{\epsilon}\right) A} \int_{\tau}^{t_{\epsilon}} e^{\left(t_{\epsilon}-s\right) B} H(\zeta(s)) d s+\int_{t_{\epsilon}}^{t} e^{(t-s) B} H(\zeta(s)) d s\right\| \leqslant \\
\leqslant C M^{2} e^{\omega\left(t-t_{\epsilon}\right)} \int_{t_{\epsilon}}^{\tau} e^{\omega\left(t_{\epsilon}-s\right)} K d s+M \epsilon \int_{t}^{t_{\epsilon}} e^{\omega(t-s)} d s \leqslant C M^{2} e^{\omega\left(t-t_{\epsilon}\right)} \frac{K}{\omega}+\epsilon \frac{M}{\omega}
\end{gathered}
$$

при $s \leqslant t_{\epsilon}$. Таким образом, для данного $\epsilon>0$ имеем

$$
0 \leqslant \limsup _{t \rightarrow-\infty}\left\|(-A)^{\theta} \int_{\tau}^{t} e^{(t-s) B} H(\zeta(s)) d s\right\| \leqslant \epsilon \frac{M}{\omega} .
$$

Таким образом, первая координата $\Theta(\eta, \zeta(t))$ лежит в $C_{0}\left((-\infty, \tau] ; P E^{\theta}\right)$. Нетрудно видеть, что вторая координата $\Theta(\eta, \zeta(t))$ лежит в $C_{0}\left((-\infty, \tau] ;(I-P) E^{\theta}\right)$.

Если положить $\eta=\eta_{0}=0$ и $\zeta(t)=\zeta_{0}(t)=\left(\begin{array}{c}v_{0}(t) \\ z_{0}(t)\end{array}\right)=0, t \in(-\infty, \tau]$, то $\zeta_{0}(t)=\Theta\left(0, \zeta_{0}(t)\right)$ и оператор $\Theta(\cdot, \cdot)$ непрерывен по обоим аргументам. Кроме того, производная Фреше $\Theta_{\zeta}^{\prime}\left(\eta_{0}, \zeta_{0}\right)$ : 
$\Upsilon \rightarrow \Upsilon$ равна

$$
\Theta_{\zeta}^{\prime}\left(\eta_{0}, \zeta_{0}\right) h=\left(\begin{array}{ll}
\int_{\tau}^{t} e^{B(t-s)} H_{v}^{\prime}\left(\zeta_{0}(s)\right) h^{1}(s) d s & \int_{\tau}^{t} e^{B(t-s)} H_{z}^{\prime}\left(\zeta_{0}(s)\right) h^{2}(s) d s \\
\int_{-\infty}^{t} e^{\tilde{A}(t-s)} G_{v}^{\prime}\left(\zeta_{0}(s)\right) h^{1}(s) d s & \int_{-\infty}^{t} e^{\tilde{A}(t-s)} G_{z}^{\prime}\left(\zeta_{0}(s)\right) h^{2}(s) d s
\end{array}\right)=0
$$

для любого $h=\left(\begin{array}{l}h^{1}(t) \\ h^{2}(t)\end{array}\right) \in \Upsilon$, поскольку производные функций $G$ и $H$ обращаются в нуль в начале координат. Следовательно, существует непрерывный оператор $\left(I-\Theta_{\zeta}^{\prime}\left(\eta_{0}, \zeta_{0}\right)\right)^{-1}$. В силу [21, теорема 54.2] заключаем, что найдутся такие $\delta, \rho>0$, что при $\left\|\eta-\eta_{0}\right\|_{Q E^{\theta}} \leqslant \delta$ уравнение (3.1.14) имеет единственное решение $\zeta(\cdot)$ в шаре $\left\|\zeta-\zeta_{0}\right\|_{\Upsilon} \leqslant \rho$, непрерывно зависящее от $\eta$.

Отсюда следует, что неустойчивое многообразие является графиком функции

$$
£:\left\{\eta \in P E^{\theta}:\|\eta\|_{E^{\theta}} \leqslant \delta\right\} \rightarrow(I-P) E^{\theta},
$$

определенной уравнением $£(\eta)=z(\tau)$, где $z(\tau)$ - вторая координата единственного решения уравнения (3.1.14). Проведенные выше рассуждения показывают также, что $£$-непрерывная функция.

Далее, предположим, что задача (1.2.16) имеет градиентную структуру.

Определение 3.1.1. Будем говорить, что полугруппа $\{S(t): t \geqslant 0\}$ является градиентной, если найдется такая непрерывная функция $V: E^{\theta} \rightarrow \mathbb{R}$, что функция $\overline{\mathbb{R}}^{+} \ni t \mapsto V\left(S(t) u^{0}\right) \in \mathbb{R}$ не возрастает и

$$
\mathcal{E}=\left\{u \in E^{\theta}: A u+f(u)=0\right\}=\left\{u^{0} \in E^{\theta}: V\left(S(t) u^{0}\right)=V\left(u^{0}\right) \forall t \in \mathbb{R}\right\} .
$$

Лемма 3.1.10. Для любого $u^{0} \in E^{\theta}$ имеем, что $\omega\left(u^{0}\right)=\left\{u^{*}\right\}$ для некоторого $u^{*} \in \mathcal{E}$. Как следствие этого факта, отметим, что $S(t) u^{0} \rightarrow u^{*}$ при $t \rightarrow \infty$.

Доказательство. Во-первых, заметим, что если $v \in \omega\left(u^{0}\right)$, то найдется такая последовательность $t_{n} \rightarrow \infty$, что $S\left(t_{n}\right) u^{0} \rightarrow v$. Поскольку функция $V\left(S(t) u^{0}\right)$ убывает и ограничена снизу, существует такое $\ell \in \mathbb{R}$, что $V\left(S(t) u^{0}\right) \rightarrow \ell$ при $t \rightarrow \infty$. Легко видеть, что $V(S(t) v)=\ell$ для всех $t \in \mathbb{R}$. Отсюда следует, что $v \in \mathcal{E}$. Поскольку множество $\mathcal{E}$ состоит из конечного числа точек и $\omega\left(u^{0}\right)$ связно, заключаем, что $\omega\left(u^{0}\right)=\left\{u^{*}\right\}$ для некоторого $u^{*} \in \mathcal{E}$.

Из следующего результата вытекает, что если существует ограниченная отрицательная орбита, проходящая через точку $u^{0}$, то $u^{0}$ лежит в неустойчивом многообразии некоторой точки $u^{*} \in \mathcal{E}$.

Лемма 3.1.11. Предположим, что точка $u^{0} \in E^{\theta}$ такова, что существует отрицательная орбита $\phi:(-\infty, 0] \rightarrow E^{\theta}$, проходящая через $u^{0}$, и замыкание $\overline{\phi((-\infty, 0])}$ компактно. Пусть

$$
\alpha_{\phi}\left(u^{0}\right)=\left\{v \in E^{\theta} \mid \exists t_{n} \rightarrow \infty: \phi\left(-t_{n}\right) \rightarrow v\right\} .
$$

Тогда существует такая точка $u^{*} \in \mathcal{E}$, что $\alpha_{\phi}\left(u^{0}\right)=\left\{u^{*}\right\}$. Кроме того, $\phi(t) \rightarrow u^{*}$ при $t \rightarrow-\infty$.

Доказательство. Во-первых, заметим, что если $v \in \alpha_{\phi}\left(u^{0}\right)$, то найдется такая последовательность $t_{n} \rightarrow \infty$, что $\phi\left(-t_{n}\right) \rightarrow v$. Поскольку $V(\phi(-t))$ возрастает и ограничена сверху, заключаем, что найдется такое $r \in \mathbb{R}$, что $V(\phi(-t)) \rightarrow r$ при $t \rightarrow \infty$. Легко видеть, что $V(S(t) v)=r$ для всех $t \in \mathbb{R}$. Отсюда следует, что $v \in \mathcal{E}$. Поскольку множество $\mathcal{E}$ состоит из конечного числа точек и $\alpha_{\phi}\left(u^{0}\right)$ связно, видим, что $\alpha_{\phi}\left(u^{0}\right)=\left\{u^{*}\right\}$ для некоторой точки $u^{*} \in \mathcal{E}$. Кроме того, для любой последовательности $t_{n} \rightarrow \infty$ последовательность $\left\{\phi\left(-t_{n}\right)\right\}$ обладает подпоследовательностью, сходящейся к $u^{*}$; следовательно, $\phi(t) \rightarrow u^{*}$ при $t \rightarrow-\infty$. 
Лемма 3.1.12. Пусть $B$-относительно компактное инвариантное подмножество пространства $E^{\theta}$ относительно $\{S(t): t \geqslant 0\}$. Если $v \in B$, то найдутся такие $v^{+}, v^{-} \in \mathcal{E}$, что $v \in W^{u}\left(v^{+}\right) u v \in W^{s}\left(v^{-}\right)$.

Доказательство. Пусть $v \in B$. Поскольку $B$ инвариантно, существует полная орбита $\mathbb{R} \ni t \mapsto$ $S(t) v \in B$, проходящая через $v$. Поскольку $B$ компактно, эта полная орбита компактна. Требуемое утверждение теперь следует из предыдущей леммы.

Из леммы 3.1.12 вытекает следующий результат.

Теорема 3.1.3 (см. [89]). Пусть $\mathcal{A}$ - аттрактор для градиентного полупотока $\{S(t): t \geqslant 0\}$ с компактной резольвентой $(\lambda I-A)^{-1}$ и пусть любая точка множества $\mathcal{E}$ является гиперболической. Тогда

$$
\mathcal{A}=\bigcup_{u^{*} \in \mathcal{E}} W^{u}\left(u^{*}\right)
$$

\section{2. АППРОКСИМАЦИЯ ОПЕРАТОРОВ ПО ОБЩЕЙ ДИСКРЕТИЗАЦИОННОЙ СХЕМЕ}

Общие примеры $\mathcal{P}$-сходимости см. в $[120,244,255,260]$.

Теорема 3.2.1. Пусть $B \in B(E)$ и $B_{n} \in B\left(E_{n}\right)$. Следуюшие условия эквивалентны:

(i) $B_{n} \stackrel{\mathcal{P P}}{\longrightarrow}$ B npu $n \rightarrow \infty$;

(ii) $\left\|B_{n}\right\| \leqslant$ const, $n \in \mathbb{N}, u\left\|B_{n} p_{n} x-p_{n} B x\right\| \rightarrow 0$ nри $n \rightarrow \infty$ для любого $x \in E$;

(iii) $\left\|B_{n}\right\| \leqslant$ const, $n \in \mathbb{N}, u\left\|B_{n} x_{n}-p_{n} B x\right\| \rightarrow 0$ nри $n \rightarrow \infty$ для любого $x \in E$, если $x_{n} \stackrel{\mathcal{P}}{\longrightarrow} x \in E$.

3.2.1. Аппроксимация спектра линейных операторов. Наиболее важную роль в аппроксимациях уравнения $B x=y$ и аппроксимациях спектра оператора $B$ играют понятия устойчивой и собственной сходимости. Эти понятия широко используются в различных областях численного анализа (см. [44,119, 124, 125, 256, 259]).

Определение 3.2.1. Будем говорить, что последовательность операторов $\left\{B_{n}\right\}, B_{n} \in B\left(E_{n}\right)$, $n \in \mathbb{N}$, устойчиво сходится к оператору $B \in B(E)$, если $B_{n} \stackrel{\mathcal{P P}}{\longrightarrow} B$ и $\left\|B_{n}^{-1}\right\|_{B\left(E_{n}\right)}=O(1)$, $n \rightarrow \infty$. Этот факт будем обозначать следующим образом: $B_{n} \stackrel{\mathcal{P P}}{\longrightarrow} B$ устойчиво.

Определение 3.2.2. Будем говорить, что последовательность операторов $\left\{B_{n}\right\}, B_{n} \in B\left(E_{n}\right)$, собственно сходится к оператору $B \in B(E)$, если $B_{n} \stackrel{\mathcal{P P}}{\longrightarrow} B$ и имеет место следующая импликация:

$$
\left\|x_{n}\right\|_{E_{n}}=O(1) \&\left\{B_{n} x_{n}\right\} \text { является } \mathcal{P} \text {-компактной } \quad \Longrightarrow \quad\left\{x_{n}\right\} \text { является } \mathcal{P} \text {-компактной. }
$$

Этот факт будем обозначать следующим образом: $B_{n} \stackrel{\mathcal{P P}}{\longrightarrow} B$ собственно.

Теорема 3.2.2 (см. [259]). Для $B_{n} \in B\left(E_{n}\right)$ и $B \in B(E)$ следующие условия эквивалентнъ:

(i) $B_{n} \stackrel{\mathcal{P P}}{\longrightarrow} B$ собственно, $B_{n}-$ фредголъмовы операторы индекса 0 и $\mathcal{N}(B)=\{0\}$;

(ii) $B_{n} \stackrel{\mathcal{P P}}{\longrightarrow}$ В устойчиво и $\mathcal{R}(B)=E$;

(iii) $B_{n} \stackrel{\mathcal{P P}}{\longrightarrow} B$ устойчиво и собственно;

(iv) если имеет место одно из условий (i)-(iii), то существуют $B_{n}^{-1} \in B\left(E_{n}\right)$ и $B^{-1} \in B(E)$ и $B_{n}^{-1} \stackrel{\mathcal{P P}}{\longrightarrow} B^{-1}$ собственно и устойчиво.

Эта теорема допускает обобщение на случай замкнутых операторов $B \in \mathcal{C}(E)$ и $B_{n} \in \mathcal{C}\left(E_{n}\right)$ (см. [261]). Пусть $\Lambda \subseteq \mathbb{C}$ - открытое связное множество и пусть $B \in B(E)$. Для изолированной точки $\lambda \in \sigma(B)$ обозначим через $\mathcal{W}(\lambda ; B)=P(\lambda) E$ соответствующее максимальное инвариантное пространство (или корневое подпространство), где

$$
P(\lambda)=\frac{1}{2 \pi i} \int_{|\zeta-\lambda|=\delta}(\zeta I-B)^{-1} d \zeta
$$


- проектор Рисса и $\delta$ достаточно мало, так что в круге $\{\zeta:|\zeta-\lambda| \leqslant \delta\}$ нет точек спектра $\sigma(B)$, отличных от $\lambda$. Изолированная точка $\lambda \in \sigma(B)$ называется точкой Pисса оператора $B$, если $\lambda I-B-$ фредгольмов оператор нулевого индекса и $P(\lambda)$ имеет конечный ранг. Пусть

$$
\mathcal{W}\left(\lambda, \delta ; B_{n}\right)=\bigcup_{\substack{\left|\lambda_{n}-\lambda\right|<\delta, \lambda_{n} \in \sigma\left(B_{n}\right)}} \mathcal{W}\left(\lambda_{n}, B_{n}\right),
$$

где $\lambda_{n} \in \sigma\left(B_{n}\right)$ выбраны из $\delta$-окрестности $\lambda$. Ясно, что $\mathcal{W}\left(\lambda, \delta ; B_{n}\right)=P_{n}(\lambda) E_{n}$, где

$$
P_{n}(\lambda)=\frac{1}{2 \pi i} \int_{|\zeta-\lambda|=\delta}\left(\zeta I-B_{n}\right)^{-1} d \zeta .
$$

В следующих теоремах установлена полная картина аппроксимации спектра.

Теорема 3.2.3 (см. $[120,257,258])$. Предположим, что $L_{n}(\lambda)=\lambda I-B_{n} u L(\lambda)=\lambda I-B-$ фредгольмови операторы нулевого индекса для любого $\lambda \in \Lambda$ и $L_{n}(\lambda) \rightarrow L(\lambda)$ устойчиво для любого $\lambda \in \rho(B) \cap \Lambda \neq \varnothing$. Тогда справедливы следующие утверждения:

(i) для любого $\lambda_{0} \in \sigma(B) \cap \Lambda$ существует такая последовательность $\left\{\lambda_{n}\right\}, \lambda_{n} \in \sigma\left(B_{n}\right), n \in \mathbb{N}$, что $\lambda_{n} \rightarrow \lambda_{0}$ nрu $n \rightarrow \infty$;

(ii) если для некоторой последовательности $\left\{\lambda_{n}\right\}, \lambda_{n} \in \sigma\left(B_{n}\right), n \in \mathbb{N}$, имеем $\lambda_{n} \rightarrow \lambda_{0} \in \Lambda$ при $n \rightarrow \infty$, mo $\lambda_{0} \in \sigma(B)$

(iii) для любого $x \in \mathcal{W}\left(\lambda_{0}, B\right)$ существует такая последовательность $\left\{x_{n}\right\}, x_{n} \in \mathcal{W}\left(\lambda_{0}, \delta ; B_{n}\right)$, $n \in \mathbb{N}$, что $x_{n} \rightarrow x$ nри $n \rightarrow \infty$;

(iv) существует такое $n_{0} \in \mathbb{N}$, что $\operatorname{dim} \mathcal{W}\left(\lambda_{0}, \delta ; B_{n}\right) \geqslant \operatorname{dim} \mathcal{W}\left(\lambda_{0}, B\right)$ для любого $n \geqslant n_{0}$.

Замечание 3.2.1. В [256] было показано, что неравенство в (iv) может быть строгим для всех $n \in \mathbb{N}$.

Теорема 3.2.4 (см. [256]). Предположим, что $L_{n}(\lambda)$ и $L(\lambda)$ - фредгольмовы операторы нулевого индекса для всех $\lambda \in \Lambda$ u $L_{n}(\lambda) \stackrel{\mathcal{P P}}{\longrightarrow} L(\lambda)$ собственно для всех $\lambda \in \Lambda$ и $\rho(B) \cap \Lambda \neq \varnothing$. Тогда справедливы утверждения (i)-(iii) теоремы 3.2 .3 и, кроме того,

$\left(\right.$ iv $\left.^{\prime}\right)$ cуществует такое $n_{0} \in \mathbb{N}$, что $\operatorname{dim} \mathcal{W}\left(\lambda_{0}, \delta ; B_{n}\right)=\operatorname{dim} \mathcal{W}\left(\lambda_{0}, B\right)$ для всех $n \geqslant n_{0}$;

(v) любал последовательность $\left\{x_{n}\right\}, x_{n} \in \mathcal{W}\left(\lambda_{0}, \delta ; B_{n}\right), n \in \mathbb{N}$, для которой $\left\|x_{n}\right\|_{E_{n}}=1$, является $\mathcal{P}$-компактной и любая ее предельная точка лежит в $\mathcal{W}\left(\lambda_{0}, B\right)$.

3.2.2. Области сходимости. Теоремы 3.2 .3 и 3.2.4 были обобщены на случай замкнутых операторов в [261]. Будем использовать следующее понятие, введенное Т. Като (см. [151]).

Определение 3.2.3. Область устойчивости $\Delta_{s}=\Delta_{s}\left(\left\{A_{n}\right\}\right), A_{n} \in \mathcal{C}\left(B_{n}\right)$, определяется как множество всех таких $\lambda \in \mathbb{C}$, что $\lambda \in \rho\left(A_{n}\right)$ для почти всех $n$, причем последовательность $\left\{\left\|\left(\lambda I-A_{n}\right)^{-1}\right\|\right\}_{n \in \mathbb{N}}$ ограничена. Область сходимости $\Delta_{c}=\Delta_{c}\left(\left\{A_{n}\right\}\right), A_{n} \in \mathcal{C}\left(E_{n}\right)$, определяется как множество всех таких $\lambda \in \mathbb{C}$, что $\lambda \in \Delta_{s}\left(\left\{A_{n}\right\}\right)$ и последовательность операторов $\left\{\left(\lambda I-A_{n}\right)^{-1}\right\}_{n \in \mathbb{N}}$ является $\mathcal{P} \mathcal{P}$-сходящейся к некоторому оператору $S(\lambda) \in B(E)$.

Определение 3.2.4. Последовательность операторов $\left\{K_{n}\right\}, K_{n} \in \mathcal{C}\left(E_{n}\right)$, называется собственно согласованной с оператором $K \in \mathcal{C}(E)$, если $\left(K_{n}, K\right)$ согласованы и для любой ограниченной последовательности $\left\|x_{n}\right\|_{E_{n}}=O(1)$, для которой $x_{n} \in D\left(K_{n}\right)$ и последовательность $\left\{K_{n} x_{n}\right\}$ является $\mathcal{P}$-компактной, имеем, что $\left\{x_{n}\right\}$ также $\mathcal{P}$-компактна, причем из $\mathcal{P}$-сходимости последовательности $\left\{x_{n}\right\}$ к некоторому $x$ и $\mathcal{P}$-сходимости последовательности $\left\{K_{n} x_{n}\right\}$ к некоторому $y$ при $n \rightarrow \infty$ в $\mathbb{N}^{\prime} \subseteq \mathbb{N}$ следует, что $x \in D(K)$ и $K x=y$.

Определение 3.2.5. Область собственной сходимости $\Delta_{r}=\Delta_{r}\left(\left\{A_{n}\right\}, A\right)$ определяется как множество всех таких $\lambda \in \mathbb{C}$, что $\left(K_{n}, K\right)$, где $K_{n}=\lambda I-A_{n}$ и $K=\lambda I-A$, собственно согласованы.

Соотношения между этими понятиями установлены в следующем предложении. 
Предложение 3.2 .1 (см. [261]). Предположим, что $\Delta_{c} \neq \varnothing$ u $\mathcal{N}(S(\lambda))=\{0\}$ по крайней мере для одной точки $\lambda \in \Delta_{c}$, так что $S(\lambda)=(\lambda I-A)^{-1}$. Тогда $A_{n}$ и $A$ согласованы и

$$
\Delta_{c}=\Delta_{s} \cap \rho(A)=\Delta_{s} \cap \Delta_{r}=\Delta_{r} \cap \rho(A) .
$$

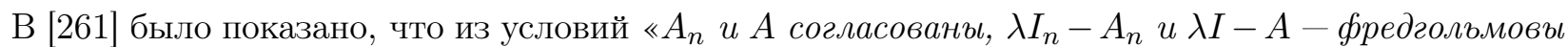
операторы нулевого индекса для любого $\lambda \in \Lambda \gg$ и $\langle\rho(A) \cap \Lambda \neq \varnothing »$ вытекают условия (i)-(iv) теоремы 3.2.3, если $\rho(A) \cap \Lambda \subseteq \Delta_{s}$, либо условия (i)-(iii) теоремы 3.2 .3 и условия (iv)-(v) of теоремы 3.2.4, если $\Lambda \subseteq \Delta_{r}$.

Определение 3.2.6. Точка Рисса $\lambda_{0} \in \sigma(A)$ называется сильно устойчивой в смысле Като, если

$$
\operatorname{dim} \mathcal{W}\left(\lambda_{0}, \delta ; B_{n}\right) \leqslant \operatorname{dim} \mathcal{W}\left(\lambda_{0}, B\right)
$$

для всех $n \geqslant n_{0}$.

Теорема 3.2.5 (см. [261]). Точка Рисса $\lambda_{0} \in \sigma(A)$ является сильно устойчивой в смысле Като тогда и толъко тогда, когда $\lambda_{0} \in \Lambda \cap \Delta_{r} \cap \sigma(A)$.

Следствие 3.2.1. Предположим, что $\Delta_{c c} \neq \varnothing$. Тогда $\Delta_{c c}=\Delta_{c} \cap \rho(A)$.

Доказательство. Ясно, что $\Delta_{c c} \subseteq \Delta_{c} \cap \rho(A)$. Чтобы доказать, что $\Delta_{c c} \supseteq \Delta_{c} \cap \rho(A)$, рассмотрим тождество Гильберта (2.3.5). Пусть $\mu \in \Delta_{c c}$. Тогда $\mu \in \Delta_{c c} \cap \Delta_{c} \cap \rho(A)$. Таким образом, для любого $\zeta \in \Delta_{c} \cap \rho(A)$ и любой ограниченной последовательности $\left\{x_{n}\right\}, n \in \mathbb{N}$, последовательность $\left\{\left(\zeta I_{n}-A_{n}\right)^{-1} x_{n}\right\}$ является $\mathcal{P}$-компактной.

Теорема 3.2.6. Предположим, что $\Delta_{c c} \neq \varnothing$. Тогда $\Delta_{r}=\mathbb{C}$.

Доказательство. Возьмем произвольную точку $\lambda_{1} \in \mathbb{C}$. Мы должны доказать, что $\lambda_{1} I-A_{n}$ и $\lambda_{1} I-A$ собственно согласованы. Предположим, что $\left\|x_{n}\right\|_{E_{n}}=O(1)$ и последовательность $\left\{\left(\lambda_{1} I_{n}-A_{n}\right) x_{n}\right\}$ является $\mathcal{P}$-компактной. Для доказательства $\mathcal{P}$-компактности последовательности $\left\{x_{n}\right\}$ возьмем $\mu \in \Delta_{c c}$. Используя (2.3.5) при $\zeta=\lambda_{1}$, получим

$$
x_{n}=\left(\mu I_{n}-A_{n}\right)^{-1}\left(\lambda_{1} I_{n}-A_{n}\right) x_{n}+\left(\lambda_{1}-\mu\right)\left(\mu I_{n}-A_{n}\right)^{-1} x_{n}
$$

и, следовательно, последовательность $\left\{x_{n}\right\}$ является $\mathcal{P}$-компактной. Предположим теперь, что $x_{n} \stackrel{\mathcal{P P}}{\longrightarrow} x$ и $\left(\lambda_{1} I_{n}-A_{n}\right)^{-1} x_{n} \stackrel{\mathcal{P}}{\longrightarrow} y$ при $n \rightarrow \infty$ в $\mathbb{N}^{\prime} \subseteq \mathbb{N}$. Тогда

$$
x=(\mu I-A)^{-1} y-\left(\lambda_{1}-\mu\right)(\mu I-A)^{-1} x,
$$

откуда следует, что $x \in D(A)$ и $\left(\lambda_{1} I-A\right) x=y$.

Из теоремы 3.2.6 следует, что в случае $\Delta_{c c} \neq \varnothing$ все предположения теоремы 3.2.4 выполнены.

Аналогично оператору $\Theta(\eta, u)$ (см. уравнение (3.1.14)) введем оператор $\Theta_{n}\left(\eta_{n}, u_{n}\right)$. Предположим, что $\eta_{n} \stackrel{\mathcal{P}^{\theta}}{\longrightarrow} \eta$, например, $\eta_{n}=p_{n}^{\theta} \eta$. Переписав уравнение (1.4.5) для новой функции $w_{n}(t)=u_{n}(t)-u_{n}^{*}$, что дает нам возможность работать в окрестности точки $u_{n}^{*}$, приходим к уравнению

$$
w_{n}^{\prime}(t)=A_{n} w_{n}(t)+f_{n}^{\prime}\left(u_{n}^{*}\right) w_{n}(t)+f_{n}\left(w_{n}(t)+u_{n}^{*}\right)-f_{n}\left(u_{n}^{*}(t)\right)-f_{n}^{\prime}\left(u_{n}^{*}\right) w_{n}(t) .
$$

Если оператор $P_{n}$ - проектор, определенный спектральным множеством

$$
\sigma_{n}^{+}=\left\{\lambda \in \sigma\left(A_{n}+f_{n}^{\prime}\left(u_{n}^{*}\right)\right): \operatorname{Re} \lambda>0\right\},
$$

и $W_{n}=P_{n} E_{n}^{\theta}$, то пространство $W_{n}$ изоморфно пространству $W=P E^{\theta}$; изоморфизм задается оператором $\Xi_{n}=P_{n} p_{n}^{\theta}$. Этот оператор является ограниченным и имеет ограниченный обратный $\Xi_{n}^{-1}$, причем нормы отображений $\Xi_{n}$ и $\Xi_{n}^{-1}$ равномерно ограничены при $n \geqslant n_{0}$. Напомним, что $P_{n}$ - такие проекторы, что $P_{n} \stackrel{\mathcal{P}^{\theta}}{\longrightarrow} P\left(\sigma^{+}\right)$компактно (определение $P\left(\sigma^{+}\right)$см. в п. 3.1.3). При помощи указанных изоморфизмов мы отождествим все пространства $W_{n}$ и $P_{n} E_{n}$ с фиксировааным пространством $W$. 
Теперь разложим уравнение (3.2.1) следующим образом. Если $w_{n}(\cdot)$-решение уравнения (3.2.1), то можем записать

$$
w_{n}(t)=v_{n}(t)+z_{n}(t), \quad \text { где } \quad v_{n}(t)=P_{n} w_{n}(t), \quad z_{n}(t)=\left(I_{n}-P_{n}\right) w_{n}(t) .
$$

Следовательно, если обозначить через $B_{n}$ ограниченный линейный оператор, определенный как сужение оператора $A_{n}$ на $P_{n} E_{n}$, получим

$$
\begin{aligned}
& v_{n}^{\prime}=B_{n} v_{n}(t)+P_{n}\left(f_{n}\left(v_{n}+z_{n}+u_{n}^{*}\right)-f_{n}\left(u_{n}^{*}\right)-f_{n}^{\prime}\left(u_{n}^{*}\right)\left(v_{n}+z_{n}\right)\right), \\
& z_{n}^{\prime}=A_{n} z_{n}+f_{n}^{\prime}\left(u_{n}^{*}\right) z+\left(I_{n}-P_{n}\right)\left(f_{n}\left(v_{n}+z_{n}+u_{n}^{*}\right)-f_{n}\left(u_{n}^{*}\right)-f_{n}^{\prime}\left(u_{n}^{*}\right)\left(v_{n}+z_{n}\right)\right) .
\end{aligned}
$$

Введем обозначения

$$
\begin{aligned}
& H_{n}\left(v_{n}, z_{n}\right)=P_{n}\left(f_{n}\left(v_{n}+z_{n}+u_{n}^{*}\right)-f_{n}\left(u_{n}^{*}\right)-f_{n}^{\prime}\left(u_{n}^{*}\right)\left(v_{n}+z_{n}\right)\right), \\
& G_{n}\left(v_{n}, z_{n}\right)=\left(I_{n}-P_{n}\right)\left(f_{n}\left(v_{n}+z_{n}+u_{n}^{*}\right)-f_{n}\left(u_{n}^{*}\right)-f_{n}^{\prime}\left(u_{n}^{*}\right)\left(v_{n}+z_{n}\right)\right) .
\end{aligned}
$$

Таким образом, имеем $H_{n}(0,0)=0$ и $G_{n}(0,0)=0$. Из непрерывной дифференцируемости функций $H_{n}$ и $G_{n}$ и оценок из раздела 3.5 , которые выполняются равномерно по $n$, следует, что для данного $\rho>0$ существуют такие $n_{0}>0$ и $\delta>0$, что если $\left\|v_{n}\right\|_{W_{n}}+\left\|z_{n}\right\|_{E_{n}^{\theta}}<\delta$ и $n \geqslant n_{0}$, то

$$
\left\{\begin{array}{l}
\left\|H_{n}\left(v_{n}, z_{n}\right)\right\|_{W_{n}} \leqslant \rho, \\
\left\|G_{n}\left(v_{n}, z_{n}\right)\right\|_{E_{n}} \leqslant \rho, \\
\left\|H_{n}\left(v_{n}, z_{n}\right)-H_{n}\left(\tilde{v}_{n}, \tilde{z}_{n}\right)\right\|_{W_{n}} \leqslant \rho\left(\left\|v_{n}-\tilde{v}_{n}\right\|_{W_{n}}+\left\|z_{n}-\tilde{z}_{n}\right\|_{E_{n}^{\theta}}\right), \\
\left\|G_{n}\left(v_{n}, z_{n}\right)-G_{n}\left(\tilde{v}_{n}, \tilde{z}_{n}\right)\right\|_{E_{n}} \leqslant \rho\left(\left\|v_{n}-\tilde{v}_{n}\right\|_{W_{n}}+\left\|z_{n}-\tilde{z}_{n}\right\|_{E_{n}^{\theta}}\right) .
\end{array}\right.
$$

Возможность выбора $\rho$ и $\delta$, удовлетворяющих приведенным выше неравенствам равномерно по $n \geqslant n_{0}$, является ключевым моментом при установлении того факта, что локально неустойчивые многообразия определены в малой «окрестности» точки равновесия $u_{n}$ равномерно при $n \geqslant n_{0}$.

Пусть $\tilde{A}_{n}=\left.\left(A_{n}+f_{n}^{\prime}\left(u_{n}^{*}\right)\right)\right|_{\left(I_{n}-P_{n}\right) E_{n}^{\theta}}$. Тогда уравнение $(3.2 .1)$ можно переписать следующим образом:

$$
\left\{\begin{array}{l}
v_{n}^{\prime}(t)=\left(A_{n}+f_{n}^{\prime}\left(u_{n}^{*}\right)\right) P_{n} v_{n}(t)+H_{n}\left(v_{n}(t), z_{n}(t)\right) \\
z_{n}^{\prime}(t)=\left(A_{n}+f_{n}^{\prime}\left(u_{n}^{*}\right)\right)\left(I_{n}-P_{n}\right) z_{n}(t)+G_{n}\left(v_{n}(t), z_{n}(t)\right)
\end{array}\right.
$$

где $H_{n}$ и $G_{n}$ удовлетворяют неравенствам (3.2.2) для всех $v_{n}(\cdot) \in W_{n}$ и $z_{n}(\cdot) \in\left(I_{n}-P_{n}\right) E_{n}^{\theta}$.

Пусть $M, \omega>0$ и

$\Omega^{M, \omega}=\left\{(v(\cdot), z(\cdot)) \in C_{0}\left((-\infty, 0] ; P E^{\theta}\right) \times C_{0}\left((-\infty, 0] ;(I-P) E^{\theta}\right):\right.$

$$
\left.\|v(t)\|_{E^{\theta}},\|z(t)\|_{E^{\theta}} \leqslant M e^{\omega t}, t \leqslant 0\right\} .
$$

Теорема 3.2.7. Оператор $\Theta(\eta, \cdot): \Omega^{M, \omega} \subset \Upsilon \rightarrow \Upsilon$ компактен.

Доказательство. Рассмотрим последовательности ограниченных функций $\left\{v^{k}(\cdot)\right\}$ и $\left\{z^{k}(\cdot)\right\}$, $v^{k}, z^{k} \in \Omega^{M, \omega}, k \in \mathbb{N}$. Тогда операторы

$$
(-A)^{\theta} \int_{0}^{t} e^{(t-s)\left(A+f^{\prime}\left(u^{*}\right)\right)} Q H\left(v^{k}(s), z^{k}(s)\right) d s, \quad(-A)^{\theta} \int_{-\infty}^{t} e^{(t-s)\left(A+f^{\prime}\left(u^{*}\right)\right)}(I-Q) G\left(v^{k}(s), z^{k}(s)\right) d s
$$

отображают эти последовательности в некоторые компактные последовательности. Действительно, легко проверить равномерную ограниченность и компактность для любого фиксированного $t<0$. Чтобы проверить равностепенную непрерывность, заметим, что

$$
\left\|(-A)^{\theta} \int_{0}^{t} e^{(t-s)\left(A+f^{\prime}\left(u^{*}\right)\right)} Q H\left(v^{k}(s), z^{k}(s)\right) d s\right\| \leqslant \epsilon
$$


если $\left\|v^{k}(s)\right\|,\left\|z^{k}(s)\right\| \leqslant \delta$ при $t \leqslant t_{\epsilon}$. Таким образом, применяя теорему Арцела-Асколи на интервале $\left[t_{\epsilon}, 0\right]$, получим равностепенную непрерывность (ср. теорему 1.4.3).

Теорема 3.2.8. Операторы

$$
\Theta_{n}\left(\eta_{n}, \cdot\right): \Omega_{n}^{M, \omega} \subset \Upsilon_{n} \rightarrow \Upsilon_{n}, \quad \Theta(\eta, \cdot): \Omega^{M, \omega} \subset \Upsilon \rightarrow \Upsilon
$$

сходятся компактно: $\Theta_{n}\left(\eta_{n}, \cdot\right) \rightarrow \Theta(\eta, \cdot)$.

Доказательство. Напомним, что

$$
\left\|u_{n}(\cdot)\right\|_{\Upsilon_{n}}=\left\|\left(u_{n}^{1}(\cdot), u_{n}^{2}(\cdot)\right)\right\|_{\Upsilon_{n}}=\sup _{-\infty<t \leqslant 0}\left\|u_{n}^{1}(t)\right\|_{P_{n} E_{n}^{\theta}}+\sup _{-\infty<t \leqslant 0}\left\|u_{n}^{2}(t)\right\|_{\left(I_{n}-P_{n}\right) E_{n}^{\theta}}
$$

и $u_{n}^{1}(-\infty)=u_{n}^{2}(-\infty)=0$. Таким образом, дискретная сходимость $\Upsilon_{n} \ni u_{n}(\cdot) \rightarrow u(\cdot) \in \Upsilon$ означает, что

$$
\sup _{-\infty<t \leqslant 0}\left\|u_{n}^{1}(t)-p_{n}^{\theta} u^{1}(t)\right\|_{P_{n} E_{n}^{\theta}}+\sup _{-\infty<t \leqslant 0}\left\|u_{n}^{2}(t)-p_{n}^{\theta} u^{2}(t)\right\|_{\left(I_{n}-P_{n}\right) E_{n}^{\theta}} \rightarrow 0 \quad \text { при } n \rightarrow \infty .
$$

Проверим, что из условия $\left\|u_{n}(\cdot)\right\| \Upsilon_{n}=O(1)$ вытекает компактность последовательности $\left\{\Theta_{n}\left(\eta_{n}, u_{n}(\cdot)\right)\right\}$. Применим теорему Арцела-Асколи к последовательностям

$$
\begin{gathered}
\left\{\left(-A_{n}\right)^{\theta} \int_{0}^{t} e^{(t-s)\left(A_{n}+f_{n}^{\prime}\left(u_{n}^{*}\right)\right)} P_{n} H_{n}\left(v_{n}^{k}(s), z_{n}^{k}(s)\right) d s\right\} \\
\left\{\left(-A_{n}\right)^{\theta} \int_{-\infty}^{t} e^{(t-s)\left(A_{n}+f_{n}^{\prime}\left(u_{n}^{*}\right)\right)}\left(I_{n}-P_{n}\right) G_{n}\left(v_{n}^{k}(s), z_{n}^{k}(s)\right) d s\right\} .
\end{gathered}
$$

Имеем

$$
\left\|\left(-A_{n}\right)^{\theta} \int_{0}^{t} e^{(t-s) A_{n}} H_{n}\left(v_{n}^{k}(s), z_{n}^{k}(s)\right) d s\right\| \leqslant \epsilon
$$

для $t \leqslant t_{\epsilon}$ в силу (3.2.2). Теперь применим теорему Арцела-Асколи к последовательностям функций

$$
\begin{gathered}
\left\{\left(-A_{n}\right)^{\theta} \int_{0}^{t} e^{(t-s)\left(A_{n}+f_{n}^{\prime}\left(u_{n}^{*}\right)\right)} P_{n} H_{n}\left(v_{n}^{k}(s), z_{n}^{k}(s)\right) d s\right\} \\
\left\{\left(-A_{n}\right)^{\theta} \int_{-\infty}^{t} e^{(t-s)\left(A_{n}+f_{n}^{\prime}\left(u_{n}^{*}\right)\right)}\left(I_{n}-P_{n}\right) G_{n}\left(v_{n}^{k}(s), z_{n}^{k}(s)\right) d s\right\}
\end{gathered}
$$

на отрезке $\left[t_{\epsilon}, 0\right]$; это делается как в теореме 1.4.3.

\section{3. АППРОКСИМАЦИЯ АТТРАКТОРОВ}

Далее будет получена сходимость аттракторов. Мы используем определение 2.1.1, чтобы сформулировать утверждения леммы 2.1.1. Эти выводы будут важны для работы с полунепрерывностью сверху и снизу.

3.3.1. Полунепрерывность аттракторов сверху. В этом пункте, используя результаты о непрерывности нелинейных полупотоков и сформулированные ниже леммы, мы докажем, что семейство аттракторов $\left\{\mathcal{A}_{n}\right\}$ полунепрерывно сверху.

Как и ранее, предположим, что операторы $A_{n}$ порождают аналитические $C_{0}$-полугруппы, удовлетворяющие оценкам

$$
\left\|e^{t A_{n}}\right\| \leqslant M e^{-t \tilde{\omega}}, \quad t \geqslant 0
$$


где $\tilde{\omega}>0$, резольвенты $\left(\lambda I_{n}-A_{n}\right)^{-1}$ компактны для некоторого $\lambda \in \rho\left(A_{n}\right)$ и сходятся компактно, $\left(\lambda I_{n}-A_{n}\right)^{-1} \rightarrow(\lambda I-A)^{-1}$ для некоторого $\lambda \in \bigcap_{n=1}^{\infty} \rho\left(A_{n}\right) \cap \rho(A)$ и $f_{n}(\cdot): E_{n}^{\theta} \rightarrow E_{n}$ - глобальнно липшицевы, ограниченные (т.е. найдется такая константа $\tilde{K}>0$, что $\left\|f_{n}(w)\right\|_{E} \leqslant \tilde{K}$ для всех $\left.w_{n} \in E_{n}^{\theta}\right)$ и непрерывно дифференцируемые по Фреше функции.

Лемма 3.3.1. Для данного $\theta \leqslant \gamma<1$ существует такая не зависящая от $n$ константа $N_{\gamma}$, чmo

$$
\sup _{n \in \mathbb{N}} \sup _{u_{n} \in \mathcal{A}_{n}}\left\|u_{n}\right\|_{E_{n}^{\gamma}} \leqslant N_{\gamma} .
$$

Кроме того, любая последовательность $\left\{u_{n}\right\}, u_{n} \in \mathcal{A}_{n}$, обладает $\mathcal{P}$-сходящейся подпоследовательностью.

Доказательство. Первое утверждение леммы непосредственно следует из инвариантности аттрактора и формулы вариации постоянной. Чтобы получить второе утверждение, достаточно применить лемму 3.5.5.

Теорема 3.3.1. Пусть $\mathcal{A}_{n}$ и $\mathcal{A}$-аттракторы задач (1.2.16) и (1.4.5) соответственно. Если последовательность $\left\{x_{n}^{*}\right\}, x_{n}^{*} \in \mathcal{A}_{n}, \mathcal{P}^{\theta}$-сходится $к$ некоторой точке $x^{*}$, то $x^{*} \in \mathcal{A} u\left\{\mathcal{A}_{n}\right\}$ является $\mathcal{P}^{\theta}$-полунепрерывной сверху на бесконечности.

Доказательство. Предположим, что $x_{n}^{*} \in \mathcal{E}_{n}$. В этом случае согласно предложению 3.3 .1 имеем $x^{*} \in \mathcal{A}$. Предположим теперь, что $x_{n}^{*}$ не принадлежит $\mathcal{E}_{n}$. Тогда $x_{n}^{*}$ может сходиться к некоторой точке в $\mathcal{E}$, и в этом случае доказывать нечего. Рассмотрим случай, когда предельная точка $x_{n}^{*} \stackrel{\mathcal{P}}{\longrightarrow} x$ не лежит в $\mathcal{E}$. В этой ситуации рассмотрим траектории, начинающиеся в этих точках при $u_{n}(0)=x_{n}^{*}$ и $u(0)=x$ в обратном направлении времени. На интервале $(-\infty, 0]$ полные траектории $u_{n}(t), t \in(-\infty, 0]$, должны быть равномерно ограничены по $n$ и $t$, поскольку функции $f_{n}(\cdot)$ равномерно ограничены по $n$ и все аттракторы $\mathcal{A}_{n}$ равномерно ограничены по $n$. По лемме 3.3 .1 любая последовательность $\left\{y_{n}\right\}, y_{n} \in \mathcal{A}_{n}, n \in \mathbb{N}$, дискретно компактна. Взяв теперь ограниченные траектории $u_{n}(\cdot)$, проходящие через $y_{n}$, и рассматривая последовательность $\left\{u_{n}(-1)\right\}$, получим, что $\left\{u_{n}(-1)\right\}$ также является дискретнно компактной. Взяв подпоследовательность $n \in \mathbb{N}^{\prime} \subset \mathbb{N}$, убеждаемся, что

Отсюда следует, что

$$
u_{n}(-1) \stackrel{\mathcal{P}^{\theta}}{\longrightarrow} \tilde{u}(-1) \quad \text { при } n \rightarrow \infty, n \in \mathbb{N}^{\prime}
$$

$$
S_{n}(t) u_{n}(-1) \rightarrow S(t) \tilde{u}(-1) \quad \text { для любого } t \in[0, T]
$$

и, в частности,

$$
S_{n}(1) u_{n}(-1)=x_{n}^{*} \stackrel{\mathcal{P}}{\longrightarrow} S(1) \tilde{u}(-1)=x .
$$

Теперь, взяв последовательность $u_{n}(-2)$, заключаем, что траектория $S(t) \tilde{u}(-2)$ ограничена при $t \geqslant 0$ совпадает с траекторией $S(t) \tilde{u}(-1)$ при $t \geqslant 0$ для общих значений $t$. Подобным образом можно построить ограниченную траекторию $u(t), t \in \mathbb{R}$, содержащую точку $x$. Таким образом, $x \in \mathcal{A}$.

3.3.2. Аппроксимация множеств равновесия. Рассмотрим следующее семейство нелинейных задач в банаховых пространствах $E_{n}$ :

$$
A_{n} u_{n}+f_{n}\left(u_{n}\right)=0
$$

Предложение 3.3.1. Предположим, что $\Delta_{c c} \neq \varnothing$ и уравнения (3.3.1) имеют решения $\left\{u_{n}^{*}\right\}$, $n \in \mathbb{N}$. Тогда, переходя при необходимости к подпоследовательностям, можно утверждать, что найдется такое решение $u^{*}$ уравнения (3.1.4), что

$$
\left\|u_{n}^{*}-p_{n}^{\theta} u^{*}\right\|_{E_{n}^{\theta}} \rightarrow 0 \quad \text { npu } n \rightarrow \infty .
$$


Доказательство. Ясно, что

$$
u_{n}^{*}+A_{n}^{-1} f_{n}\left(u_{n}^{*}\right)=0 .
$$

Поскольку $A_{n}^{-1} \stackrel{\mathcal{P P}}{\longrightarrow} A^{-1}$ компактно по лемме 3.5 .5 и функции $f_{n}(\cdot)$ ограничены равномерно по $n \in \mathbb{N}$, заключаем, что найдутся такие подпоследовательность (обозначим ее также символом $u_{n}^{*}$ ) и точка $u^{*}$, что $u_{n}^{*} \stackrel{\mathcal{P}^{\theta}}{\longrightarrow} u^{*}$. Из непрерывности функций $f_{n}(\cdot)$ и сходимости резольвент $A_{n}^{-1} \stackrel{\mathcal{P P}}{\longrightarrow} A^{-1}$ следует, что $u^{*}+A^{-1} f^{*}\left(u^{*}\right)=0$; это эквивалентно тому факту, что $u^{*}$ - решение уравнения (3.1.4).

Предложение 3.3.2. Предположим, что $A_{n}^{-1} \stackrel{\mathcal{P P}}{\longrightarrow} A^{-1}$ компактно и $f_{n}\left(x_{n}\right) \stackrel{\mathcal{P}}{\longrightarrow} f(x)$ при $x_{n} \stackrel{\mathcal{P}^{\theta}}{\longrightarrow} x$. Предположим такюе, что $u^{*}$ - гиперболическое решение уравнения (3.1.4). Тогда существует такое $n_{0}$, что при $n \geqslant n_{0}$ уравнения (3.3.1) имеют по крайней мере одну последовательность решений $\left\{u_{n}^{*}\right\}$. Любал последовательность $\left\{u_{n}^{*}\right\}, n \in \mathbb{N}$, является $\mathcal{P}^{\theta}$-компактной, и $\mathcal{P}^{\theta}$-предельная точка последовательности $\left\{u_{n}^{*}\right\}, n \in \mathbb{N}$, есть решение $u^{*}$ уравнения (3.1.4), m.e. $u_{n}^{*} \stackrel{\mathcal{P}^{\theta}}{\longrightarrow} u^{*}$ npu $n \in \mathbb{N}^{\prime} \subset \mathbb{N}$.

Доказательство. Как и в теореме 3.1.1, существует шар $\mathcal{U}\left(u^{*}, \delta\right)$, не содержащий неподвижных точек, кроме $u^{*}$, и мы имеем $\left|\operatorname{ind}\left(u^{*}, I+A^{-1} f(\cdot)\right)\right|=1$. Компактная сходимость

$$
A_{n}^{-1} f_{n}(\cdot) \stackrel{\mathcal{P P}}{\longrightarrow} A^{-1} f(\cdot)
$$

следует из ограниченности и непрерывности функций $f_{n}(\cdot)$ и компактной сходимости $A_{n}^{-1} \stackrel{\mathcal{P P}}{\longrightarrow} A^{-1}$. Теперь из компактной сходимости $A_{n}^{-1} f_{n}(\cdot) \rightarrow A^{-1} f(\cdot)$ получаем (см. [259, теорема 3]), что

$$
\text { ind }\left(u^{*}, I+A^{-1} f(\cdot)\right)=\gamma\left(I_{n}+A_{n}^{-1} f_{n}(\cdot), \partial \mathcal{U}\left(p_{n} u^{*}, \delta\right)\right), \quad n \geqslant n_{0} .
$$

Это означает, что в любом шаре $\mathcal{U}\left(p_{n} u^{*}, \delta\right), n \geqslant n_{0}$, существует по крайней мере одна неподвижная точка $u_{n}^{*}$. Эта последовательность $\left\{u_{n}^{*}\right\}$ является $\mathcal{P}^{\theta}$-компактной и сходится на подпоследовательностях к $u^{*}$.

В случае, когда известна дифференцируемость соответствующих функций, имеем более строгий результат.

Предложение 3.3.3. Предположим, что уравнение (3.1.4) имеет решение и*, являющееся гиперболической точкой. Предположим также, что в шарах, построенных вокруг точек $p_{n}^{\theta} u^{*}$, существуют производные Фреше функиий $f_{n}(\cdot)$, причем $f_{n}^{\prime}\left(u_{n}\right)$ равномерно непрерывны по $n$ в шарах $\mathcal{U}\left(p_{n}^{\theta} u^{*}, \delta\right)$ для некоторого $\delta>0$. Тогда существуют такие $n_{0} u \delta>0$, что уравнения (3.3.1) имеют единственные решения $u_{n}^{*}$ в $\left\{w_{n}:\left\|w_{n}-p_{n}^{\theta} u^{*}\right\|_{E_{n}^{\theta}} \leqslant \delta\right\}$ при $n \geqslant n_{0}$. Кроме mого,

$$
\left\|u_{n}^{*}-p_{n}^{\theta} u^{*}\right\|_{E_{n}^{\theta}} \rightarrow 0 \quad \text { npu } n \rightarrow \infty .
$$

Доказательство. Докажем, что в окрестности решения $u^{*}$ уравнения (3.1.4) найдется единственное решение $u_{n}^{*}$ уравнения (3.3.1). Кроме того, поскольку $f_{n}^{\prime}(\cdot)$ равномерно непрерывны по $n$, найдется такое $\delta>0$, что из неравенства

$$
\left\|u_{n}^{*}-p_{n}^{\theta} u^{*}\right\|_{E_{n}^{\theta}}<\delta
$$

следует

$$
M\left\|f_{n}^{\prime}\left(u_{n}^{*}\right)-f_{n}^{\prime}\left(p_{n}^{\theta} u^{*}\right)\right\|_{B\left(E_{n}^{\theta}, E_{n}\right)}<\frac{1}{(2 C)} .
$$

По лемме 3.5.6, $\sigma\left(A_{n}+f_{n}^{\prime}\left(p_{n}^{\theta} u^{*}\right)\right)$ не пересекается с мнимой осью и найдется такая константа $M>0$, что

$$
\left\|\left(A_{n}+f_{n}^{\prime}\left(p_{n}^{\theta} u^{*}\right)\right)^{-1}\right\|_{B\left(E_{n}, E_{n}^{\theta}\right)} \leqslant M .
$$


Если $u_{n}$ - решение уравнения (3.3.1), то

$$
\begin{aligned}
0=A_{n} u_{n}+f_{n}^{\prime}\left(p_{n}^{\theta} u^{*}\right) u_{n}+ & f_{n}\left(u_{n}\right)-f_{n}^{\prime}\left(p_{n}^{\theta} u^{*}\right) u_{n}= \\
= & \left(A_{n}+f_{n}^{\prime}\left(p_{n}^{\theta} u^{*}\right)\right)\left(u_{n}+\left(A_{n}+f_{n}^{\prime}\left(p_{n}^{\theta} u^{*}\right)\right)^{-1}\left(f_{n}\left(u_{n}\right)-f_{n}^{\prime}\left(p_{n}^{\theta} u^{*}\right) u_{n}\right)\right) .
\end{aligned}
$$

Поскольку $\left(A_{n}+f_{n}^{\prime}\left(p_{n}^{\theta} u^{*}\right)\right)$ обратим, доказательство того факта, что $u_{n}$-решение уравнения (3.3.1), эквивалентно доказательству того факта, что $u_{n}$ является неподвижной точкой отображения

$$
\Phi_{n}\left(u_{n}\right)=-\left(A_{n}+f_{n}^{\prime}\left(p_{n}^{\theta} u^{*}\right)\right)^{-1}\left(f_{n}\left(u_{n}\right)-f_{n}^{\prime}\left(p_{n}^{\theta} u^{*}\right) u_{n}\right) .
$$

Из сходимости резольвент и непрерывности $f_{n}(\cdot)$ и $f_{n}^{\prime}\left(p_{n}^{\theta} u^{*}\right)$ следует, что

$$
\Phi_{n}\left(p_{n}^{\theta} u^{*}\right) \rightarrow-\left(A+f^{\prime}\left(u^{*}\right)\right)^{-1}\left(f\left(u^{*}\right)-f^{\prime}\left(u^{*}\right) u^{*}\right)=u^{*} .
$$

Далее, покажем, что для достаточно малого $\delta>0$ и $n \geqslant n_{0}$ отображение $\Phi_{n}$ является сжимающим отображением из замкнутого шара $\overline{\mathcal{U}}\left(p_{n} u^{*}, \delta\right)$ в себя. Во-первых, проверим, что $\Phi_{n}-$ сжимающее отображение (равномерно по $n \in \mathbb{N}$ ), т.е.

$$
\begin{gathered}
\left\|\Phi_{n}\left(u_{n}\right)-\Phi_{n}\left(v_{n}\right)\right\|_{E_{n}^{\theta}}=\left\|\left(A_{n}+f_{n}^{\prime}\left(p_{n}^{\theta} u^{*}\right)\right)^{-1}\left(f_{n}\left(u_{n}\right)-f_{n}\left(v_{n}\right)-f_{n}^{\prime}\left(p_{n}^{\theta} u^{*}\right)\left(u_{n}-v_{n}\right)\right)\right\|_{E_{n}^{\theta}} \leqslant \\
\leqslant\left\|\left(A_{n}+f_{n}^{\prime}\left(p_{n}^{\theta} u^{*}\right)\right)^{-1}\right\|_{L\left(E_{n}, E_{n}^{\theta}\right)}\left\|f_{n}\left(u_{n}\right)-f\left(v_{n}\right)-f_{n}^{\prime}\left(p_{n}^{\theta} u^{*}\right)\left(u_{n}-v_{n}\right)\right\|_{E_{n}} \leqslant \\
\leqslant M\left\|\left(f_{n}^{\prime}\left(s u_{n}+(1-s) v_{n}\right)-f_{n}^{\prime}\left(p_{n} u^{*}\right)\right)\left(u_{n}-v_{n}\right)\right\|_{E_{n}} \leqslant \\
\leqslant M\left\|f_{n}^{\prime}\left(s u_{n}+(1-s) v_{n}\right)-f_{n}^{\prime}\left(p_{n}^{\theta} u^{*}\right)\right\|_{L\left(E_{n}^{\theta}, E_{n}\right)}\left\|u_{n}-v_{n}\right\|_{E_{n}^{\theta}} \leqslant \frac{1}{2 C}\left\|u_{n}-v_{n}\right\|_{E_{n}^{\theta}}
\end{gathered}
$$

для некоторого $0 \leqslant s \leqslant 1$ и $n \geqslant n_{0}$. Чтобы показать, что $\Phi_{n}$ отображает $\mathcal{U}\left(p_{n} u^{*}, \delta\right)$ в себя, заметим, что если $u_{n} \in \mathcal{U}\left(p_{n} u^{*}, \delta\right)$, то

$$
\begin{aligned}
\left\|\Phi_{n}\left(u_{n}\right)-p_{n} u^{*}\right\|_{E_{n}^{\theta}} \leqslant\left\|\Phi_{n}\left(u_{n}\right)-\Phi_{n}\left(p_{n} u^{*}\right)\right\|_{E_{n}^{\theta}}+\left\|\Phi_{n}\left(p_{n} u^{*}\right)-p_{n} u^{*}\right\|_{E_{n}^{\theta}} & \leqslant \\
& \leqslant \frac{\delta}{2}+\left\|\Phi_{n}\left(p_{n} u^{*}\right)-p_{n} u^{*}\right\|_{E_{n}^{\theta}} .
\end{aligned}
$$

Из (3.3.2) следует, что существует такое $n_{0}$, что

$$
\left\|\Phi_{n}\left(u_{n}\right)-p_{n} u^{*}\right\|_{E_{n}^{\theta}} \leqslant \frac{3 \delta}{4} \quad \text { при } n \geqslant n_{0} .
$$

Отсюда следует, что если $u_{n} \in \overline{\mathcal{U}}\left(p_{n}^{\theta} u^{*}, \delta\right)$, то

$$
\left\|\Phi_{n}\left(u_{n}\right)-p_{n}^{\theta} u^{*}\right\|_{E_{n}^{\theta}} \leqslant \delta
$$

и, следовательно, $\Phi_{n}: \mathcal{U}\left(p_{n} u^{*}, \delta\right) \rightarrow \mathcal{U}\left(p_{n} u^{*}, \delta\right)$ - сжимающее отображение для всех $n \geqslant n_{0}$. Поэтому в шаре $\mathcal{U}\left(p_{n}^{\theta} u^{*}, \delta\right)$ имеется единственная неподвижная точка уравнения (3.3.1).

Для доказательства сходимости $u_{n}^{*} \stackrel{\mathcal{P}^{\theta}}{\longrightarrow} u^{*}$ поступим следующим образом:

$$
\left\|p_{n}^{\theta} u^{*}-u_{n}^{*}\right\|_{E_{n}^{\theta}}=\left\|\Phi_{n}\left(u_{n}^{*}\right)-p_{n} u^{*}\right\|_{E_{n}^{\theta}} \leqslant\left\|\Phi_{n}\left(u_{n}^{*}\right)-\Phi_{n}\left(p_{n} u^{*}\right)\right\|_{E_{n}^{\theta}}+\left\|\Phi_{n}\left(p_{n} u^{*}\right)-p_{n} u^{*}\right\|_{E_{n}^{\theta}} .
$$

Из того факта, что $\Phi_{n}$ - равномерно сжимающее отображение в $E_{n}^{\theta}$, получаем

$$
\left.\left\|p_{n}^{\theta} u^{*}-u_{n}^{*}\right\|_{E_{n}^{\theta}} \leqslant 2 \| \Phi_{n}\left(p_{n}^{\theta} u^{*}\right)-p_{n}^{\theta} u^{*}\right) \|_{E_{n}^{\theta}} .
$$

Теперь из (3.3.2) вытекает требуемая сходимость.

В качестве непосредственного следствия предложения 3.3 .3 получаем следующую теорему.

Теорема 3.3.2. Предположим, что уравнение (3.1.4) имеет в точности $m$ решений (точек равновесия) $u^{1}, \ldots, u^{m}$, причем все гиперболические. Предположим, что для любой точки $u^{i} u$ любого $\epsilon>0$ существует такое $\delta>0$, что

$$
\left\|f_{n}^{\prime}\left(u_{n}\right)-f_{n}^{\prime}\left(p_{n}^{\theta} u^{i}\right)\right\|_{E_{n}} \leqslant \epsilon, \quad \text { еслu }\left\|u_{n}-p_{n}^{\theta} u^{i}\right\|_{E_{n}^{\theta}} \leqslant \delta .
$$


Тогда существует такое $n_{0}$, что для всех $n \geqslant n_{0}$ уравнения (3.3.1) имеют ровно т решений $u_{n}^{1}, \ldots, u_{n}^{m}$. Кроме того,

$$
\left\|u_{n}^{k}-p_{n}^{\theta} u^{k}\right\|_{E_{n}^{\theta}} \rightarrow 0 \quad \text { при } n \rightarrow \infty \text { для любого } k=1, \ldots, m .
$$

Доказательство. Согласно предложению 3.3.1 имеем, что любое решение $u_{n}^{k}$ уравнения (3.3.1), $n \rightarrow \infty$, лежит в окрестности множества точек равновесия уравнения (3.1.4). Согласно предложению 3.3.3, окрестность точки $u^{k}$ содержит лишь одно решение уравнения (3.3.1), которое сходится к $u^{k}$, что и доказывает требуемый результат.

\section{4. АППРОКСИМАЦИЯ НЕУСТОЙЧИВЫХ МНОГООБРАЗИЙ}

Напомним, что мы предположили, что все равновесные решения задачи (1.2.16) являются гиперболическими. Предположим также, что каждое решение $u^{*}$ уравнения (3.1.4) таково, что спектр $\sigma\left(A+f^{\prime}\left(u^{*}\right)\right)$ оператора $A+f^{\prime}\left(u^{*}\right)$ не пересекается с мнимой осью.

Предложение 3.4.1. Пусть $A$-секториалъный оператор с компактной резольвентой $и$ $f^{\prime}\left(u^{*}\right) \in B\left(E^{\theta}, E\right)$. Тогда оператор $\left(A+f^{\prime}\left(u^{*}\right)\right)$ также является секториалъным с компактной резольвентой.

Предложение 3.4.1 непосредственно следует из [136, теоремы 1.3.2 и 1.4.8].

Из предложения 3.4 .1 следует, что

$$
\sigma^{+}=\sigma\left(\left(A+f^{\prime}\left(u^{*}\right)\right)\right) \cap\{\lambda \in \mathbb{C}: \operatorname{Re} \lambda>0\}
$$

состоит из конечного числа (возможно, нулевого) собственных значений конечной кратности, причем найдутся такие $\beta>0$ и проектор $P\left(\sigma^{+}\right)$конечного ранга, что

$$
\begin{cases}\left\|e^{\left(A+f^{\prime}\left(u^{*}\right)\right) t} P\right\|_{B(E)} \leqslant M e^{\beta t}, & t \leqslant 0, \\ \left\|e^{\left(A+f^{\prime}\left(u^{*}\right) t\right.}(I-P)\right\|_{B\left(E, E^{\theta}\right)} \leqslant M t^{-\theta} e^{-\beta t}, & t \geqslant 0, \theta \geqslant 0 .\end{cases}
$$

Предложение 3.4.2. Пусть $u_{n}^{*} \stackrel{\mathcal{P}^{\theta}}{\longrightarrow} u^{*}$, где $u_{n}^{*}-$ решение уравнения (3.3.1) и $u^{*}-$ гиперболическое решение уравнения (3.1.4). Предположим, что

$$
\left(\lambda I_{n}-A_{n}\right)^{-1} \stackrel{\mathcal{P P}}{\longrightarrow}(\lambda I-A)^{-1} \text { компактно. }
$$

Тогда найдется такое $n \geqslant n_{0}$, что $\sigma\left(A_{n}+f_{n}^{\prime}\left(u_{n}^{*}\right)\right)$ не пересекается с мнимой осъю при $n \geqslant n_{0} u$

$$
\left\|\left(A_{n}+f_{n}^{\prime}\left(u^{*}\right)\right)^{-1}\right\| \leqslant C
$$

где $C$ не зависит от $n$. Кроме того, если $P_{n}$ - проектор, определенный спектральным множеством

$$
\sigma_{n}^{+}=\left\{\lambda \in \sigma\left(A_{n}+f_{n}^{\prime}\left(u_{n}^{*}\right)\right): \operatorname{Re} \lambda>0\right\}
$$

то $P_{n}$ сходится компактно к $P$ при $n \rightarrow \infty\left(\right.$ mаким образом, $\left.\operatorname{rank}\left(P_{n}\right)=\operatorname{rank}(P) n p u n \geqslant n_{0}\right) u$ семейство множеств $\sigma_{n}^{+}$сходится, $\sigma_{n}^{+} \rightarrow \sigma^{+}$, в смысле условий (i)-(iii) теоремъ 3.2 .3 и условий (iv)-(v) теоремы 3.2.4.

Предложение 3.4.2 вытекает из леммы 3.5.6 и теоремы 3.2.4.

Предложение 3.4.3. Предположим, что

$$
\left(\lambda I_{n}-A_{n}\right)^{-1} \stackrel{\mathcal{P} \mathcal{P}}{\longrightarrow}(\lambda I-A)^{-1} \text { компактно. }
$$

Тогда для достаточно большого $n$ равновесие $u_{n}^{*}$ уравнения (3.3.1) является гиперболическим и найдется такое $\beta>0$, что если $P_{n}-$ проектор, определенная спектральным множеством $\sigma_{n}^{+}$, mo

$$
\begin{cases}\left\|e^{t\left(A_{n}+f_{n}^{\prime}\left(u_{n}^{*}\right)\right)} P_{n}\right\|_{B\left(E_{n}\right)} \leqslant M e^{\beta t}, & t \leqslant 0, \\ \left\|e^{t\left(A_{n}+f_{n}^{\prime}\left(u^{*}\right)\right)}\left(I_{n}-P_{n}\right)\right\|_{B\left(E_{n}, E_{n}^{\theta}\right)} \leqslant M t^{-\theta} e^{-\beta t}, & t \geqslant 0, \quad \theta \geqslant 0 .\end{cases}
$$

Доказательство предложения 3.4.3 аналогично доказательству теоремы 3.5.3. 
Теорема 3.4.1. Пусть $\Delta_{c c} \neq \varnothing u u^{*}$ - гиперболическая точка равновесия задачи (1.2.16). Тогда для достаточно малого $\delta>0$ уравнение (3.1.14) имеет такое решение $\zeta^{*}(\cdot)$, что уравнения

$$
\zeta_{n}(t)=\Theta_{n}\left(\eta_{n}, \zeta_{n}(t)\right)
$$

имеют решения $\zeta_{n}^{*}(\cdot), n \geqslant n_{0}$, причем $\zeta_{n}^{*}(t) \rightarrow \zeta^{*}(t)$ равномерно по $t \in(-\infty, 0]$ при $n \rightarrow \infty$.

Доказательство. Известно, что $\left|\operatorname{ind}\left(\zeta_{0}, I-\Theta\right)\right|=1$ при $\zeta_{0}=0$. Согласно [21, теорема 54.1], существуют такие $\rho$ и $\delta>0$, что $|\operatorname{ind}(\zeta, I-\Theta)|=1$ при $\|\eta-0\| \leqslant \delta$ и $\|\zeta-0\| \leqslant \rho$. Согласно теореме 3.2 .8 видим, что $\Theta_{n} \rightarrow \Theta$ компактно, так что $\gamma\left(I_{n}-\Theta_{n}, \partial \Omega_{n}^{M, \omega}\right)=\operatorname{ind}(\zeta, I-\Theta)$ и, следовательно, уравнение

$$
\zeta_{n}(t)=\Theta_{n}\left(\eta_{n}, \zeta_{n}(t)\right)
$$

имеет по крайней мере одно решение $\zeta_{n}^{*}$ в любом множестве $\Omega_{n}^{M, \omega}, n \geqslant n_{0}$. Последовательность $\left\{\zeta_{n}^{*}\right\}$ дискретно компактна и $\zeta_{n}^{*} \rightarrow \zeta^{*}$ при $n \rightarrow \infty$.

Предложение 3.4.4. Предположим также, что $u^{*}$ - гиперболическое равновесие задачи (1.2.16) и нуль не принадлежит спектру оператора $A+f^{\prime}\left(u^{*}\right): D(A) \subset E \rightarrow E$. Предположим, что

$$
\left(\lambda I_{n}-A_{n}\right)^{-1} \stackrel{\mathcal{P P}}{\longrightarrow}(\lambda I-A)^{-1} \text { компактно }
$$

$u\left\|f_{n}\left(u_{n}\right)-f_{n}^{\prime}\left(p_{n}^{\theta} u^{*}\right)\right\|$ стремится $\kappa$ нулю равномерно по $n$, т.е. сходится $\kappa$ нулю при $\left\|u_{n}-p_{n}^{\theta} u^{*}\right\|_{E_{n}^{\theta}} \rightarrow 0$. Тогда существуют такие $\delta$ u $n_{0}>0$, что $u_{n}^{*}$ имеет локальное неустойчивое многообразие $W_{l o c}^{u}\left(u_{n}^{*}\right) \subset E_{n}^{\theta}$ при $n \geqslant n_{0}$. Если ввести обозначение

$$
W_{n, \delta}^{u}\left(u_{n}^{*}\right)=\left\{w_{n} \in W_{l o c}^{u}\left(u_{n}^{*}\right):\left\|w_{n}-u_{n}^{*}\right\|_{E_{n}^{\theta}}<\delta\right\}, \quad n \geqslant n_{0},
$$

mo $W_{n, \delta}^{u}\left(u_{n}^{*}\right)$ сходится $\kappa W_{\delta}^{u}\left(u^{*}\right)$ nрu $n \rightarrow \infty$, m.e.

$$
\sup _{w_{n} \in W_{n, \delta}^{u}\left(u_{n}^{*}\right)} \inf _{w \in W_{\delta}^{u}\left(u^{*}\right)}\left\|w_{n}-p_{n}^{\theta} w\right\|_{E_{n}^{\theta}}+\sup _{w \in W_{\delta}^{u}\left(u^{*}\right)} \inf _{w_{n} \in W_{n, \delta}^{u}\left(u_{n}^{*}\right)}\left\|w_{n}-p_{n}^{\theta} w\right\|_{E_{n}^{\theta}} \rightarrow 0 \quad \text { npu } n \rightarrow \infty .
$$

Доказательство. Заметим, что согласно предложению 3.3.3, уравнения (3.3.1) имеют такие единственные решения $u_{n}^{*}$ вблизи $u^{*}$, что $\left\|u_{n}^{*}-p_{n}^{\theta} u^{*}\right\|_{E_{n}^{\theta}} \rightarrow 0$ при $n \rightarrow \infty$. Согласно предложению 3.4 .2 отсюда следует, что спектр оператор $A_{n}+f_{n}^{\prime}\left(u_{n}^{*}\right)$ непрерывен при $n \rightarrow \infty$.

Кроме того, для некоторого положительного $M$ и $\beta$, не зависящего от $n$, согласно предложению 3.4 .3 имеем

$$
\begin{array}{ll}
\left\|e^{\tilde{A}_{n} t} z_{n}\right\|_{E_{n}^{\theta}} \leqslant M e^{-\beta t}\left\|z_{n}\right\|_{E_{n}^{\theta}}, & t \geqslant 0, \\
\left\|e^{\tilde{A}_{n} t} z_{n}\right\|_{E_{n}^{\theta}} \leqslant M t^{-\theta} e^{-\beta t}\left\|z_{n}\right\|_{E_{n}}, & t>0, \\
\left\|e^{B_{n} t} v_{n}\right\|_{W_{n}} \leqslant M e^{\beta t}\left\|v_{n}\right\|_{W_{n}}, & t \leqslant 0 .
\end{array}
$$

Покажем теперь, что для достаточно малого $\rho>0$ найдется неустойчивое многообразие для $u_{n}^{*}$

$$
W_{n}^{u}=\left\{\left(v_{n}(\cdot), z_{n}(\cdot)\right): z_{n}(\cdot) \in\left(I_{n}-P_{n}\right) E_{n}^{\theta}, v_{n}(\cdot) \in W_{n}\right\} .
$$

Как и в теореме 3.1.2, введем операторы $\Theta_{n}$ и вектор $\zeta_{n}(t)=\left(\begin{array}{l}v_{n}(t) \\ z_{n}(t)\end{array}\right)$. Из неравенств $(3.2 .2)$ получим, что найдется такое положительное $\delta$, что операторы $\Theta_{n}\left(\eta_{n}, \cdot\right)$ дифференцируемы по Фреше в шарах $\mathcal{U}\left(p_{n}^{\theta} \zeta^{*}, \delta\right)$ и для любого $\epsilon>0$ найдется такое $\delta_{\epsilon}>0$, что

$$
\left\|\Theta_{n}^{\prime}\left(\eta_{n}, \zeta_{n}\right)-\Theta_{n}^{\prime}\left(\eta_{n}, p_{n}^{\theta} \zeta^{*}\right)\right\| \leqslant \epsilon \quad \text { при }\left\|\zeta_{n}(\cdot)-p_{n}^{\theta} \zeta^{*}(\cdot)\right\|_{\Upsilon_{n}} \leqslant \delta_{\epsilon} .
$$

Ясно также, что условия

$$
\left\|p_{n}^{\theta} \zeta^{*}(\cdot)-\Theta_{n}\left(\eta_{n}, p_{n}^{\theta} \zeta^{*}(\cdot)\right)\right\|_{\Upsilon_{n}} \rightarrow 0, \quad\left\|\left(I_{n}-\Theta_{n}^{\prime}\left(\eta_{n}, p_{n}^{\theta} \zeta^{*}(\cdot)\right)\right)^{-1}\right\| \leqslant \text { const }
$$

выполнены при $n \rightarrow \infty$. Тогда согласно [259, теорема 2] заключаем, что существуют такие $n_{0} \in \mathbb{N}$ и $0<\delta_{0} \leqslant \delta$, что уравнения (3.2.3) имеют единственое решение $\zeta_{n}^{*}(\cdot)$ в шаре $\mathcal{U}\left(p_{n}^{\theta} \zeta^{*}, \delta_{0}\right)$ при $n \geqslant n_{0}$ и $\zeta_{n}^{*} \rightarrow \zeta^{*}$ при $n \rightarrow \infty$. 
Из предложения 3.4.4 непосредственно вытекает следующее утверждение.

Следствие 3.4.1. Предположим, что условия предложения 3.4 .4 выполнены, уравнение (3.1.4) имеет в точности $m$ решений $u^{1}, \ldots, u^{m}$, причем все они являются гиперболическими. Тогда существует такое достаточно малое $\delta>0$, что уравнение (3.3.1) имеет ровно $m$ решений и их локальные неустойчивые многообразия $W_{\delta}^{u}\left(u_{n}^{k}\right), k=1, \ldots, m$, обнаруживают непрерывное поведение в $E_{n}^{\theta}$ при $n \rightarrow \infty$.

\subsection{1. Полунепрерывность аттракторов снизу.}

Теорема 3.4.2. Пусть $\mathcal{A}$ и $\mathcal{A}_{n}$ - аттракторы для задач (1.2.16) и (1.4.5) соответственно. Предположим, что задача (1.2.16) имеет градиентную структуру и все точки равновесия являются гиперболическими. Если $x^{*} \in \mathcal{A}$, тогда существует такая последовательность точек $\left\{x_{n}^{*}\right\}, x_{n}^{*} \in \mathcal{A}_{n}$, что $x_{n}^{*} \stackrel{\mathcal{P}^{\theta}}{\longrightarrow} x^{*}$ и последовательность аттракторов $\left\{\mathcal{A}_{n}\right\}$ является $\mathcal{P}^{\theta}-$ полунепрерывной снизу на бесконечности.

Доказательство. Предположим, что $x^{*} \in \mathcal{E}$. Утверждение теоремы следует из предложения 3.3.2. Действительно, предположим, что $x^{*}$ лежит на неустойчивом многообразии. Тогда, двигаясь в обратном направлении по времени вдоль некоторой траектории из точки $x^{*}$, можно прийти в некоторую точку $y$ в окрестности некоторой точки равновесия, которая, как известно из теоремы 3.4.1, аппроксимируется точками $y_{n} \in \mathcal{A}_{n}$. Теперь, стартуя из точек $y_{n}$, согласно теореме 2.3.3, получим утверждение теоремы 3.4.2.

\section{5. ВСПОМОГАТЕЛЬНЫЕ РЕЗУЛЬТАТЫ}

Лемма 3.5.1. Предположим, что $B_{n} \stackrel{\mathcal{P} \mathcal{P}}{\longrightarrow} B$ компактно, операторъ $B$ и $B_{n}$ компактны $u$ $\mathcal{N}(I+B)=\{0\}$. Тогда существуют такие $n_{0}>0$ и $M>0$, что

$$
\left\|\left(I_{n}+B_{n}\right)^{-1}\right\|_{B\left(E_{n}\right)} \leqslant M, \quad n \geqslant n_{0} .
$$

Доказательство. Поскольку из компактной сходимости $B_{n} \stackrel{\mathcal{P} \mathcal{P}}{\longrightarrow} B$ следует собственная сходимость $I_{n}+B_{n} \stackrel{\mathcal{P} \mathcal{P}}{\longrightarrow} I+B$, требуемый результат вытекает из теоремы 3.2.2.

Лемма 3.5.2. Пусть $\Delta_{c c} \neq \varnothing$. Тогда для любого $\lambda \in \rho(A)$ найдется такое $n_{\lambda}>0$, что $\lambda \in \rho\left(A_{n}\right)$ для всех $n \geqslant n_{\Lambda}$, причем существует такая константа $M_{\lambda}>0$, что

$$
\left\|\left(\lambda I_{n}-A_{n}\right)^{-1}\right\| \leqslant M_{\lambda}, \quad n \geqslant n_{\Lambda} .
$$

Кроме того, $\left(\lambda I_{n}-A_{n}\right)^{-1} \stackrel{\mathcal{P P}}{\longrightarrow}(\lambda I-A)^{-1}$ компактно.

Доказательство. Требуемый результат вытекает из леммы 3.5.1 и следствия 3.2.1.

Лемма 3.5.3. Предположим, что $\Delta_{c c} \neq \varnothing$ и резольвенты $(\lambda I-A)^{-1} u\left(\lambda I_{n}-A_{n}\right)^{-1}$ компактнъ. Пусть $\Lambda$ - компактное подмножество множества $\rho(A)$. Тогда найдется такая константа $n_{\Lambda}>0$, что $\Lambda \subset \rho\left(A_{n}\right)$ для всех $n \geqslant n_{\Lambda} u$

$$
\sup _{\substack{\lambda \in \Lambda, n \geqslant n_{\Lambda}}}\left\|\left(\lambda I_{n}-A_{n}\right)^{-1}\right\|<\infty .
$$

Кроме того, для любого $и \in E$ имеем

$$
\sup _{\lambda \in \Lambda}\left\|\left(\lambda I_{n}-A_{n}\right)^{-1} p_{n} u-p_{n}(\lambda I-A)^{-1} u\right\| \rightarrow 0 .
$$

Доказательство. Во-первых, докажем, что найдется такое $n_{\Lambda}>0$, что $\Lambda \subset \rho\left(A_{n}\right)$ для всех $n \geqslant n_{\Lambda}$. Предположим противное; тогда найдутся такие последовательности $k_{n} \rightarrow \infty$ и $\left\{\lambda_{k_{n}}\right\} \in \Lambda$, что $\lambda_{k_{n}}$ - собственное значение оператора $A_{k_{n}}$. Так как $\Lambda$ компактно, можно предположить, что найдется такое $\bar{\lambda} \in \Lambda$, что $\lambda_{k_{n}} \rightarrow \bar{\lambda}$. Из теоремы 3.2.3(ii) теперь следует, что $\bar{\lambda} \in \sigma(A)$, противоречие. 
Для доказательства (3.5.2) достаточно убедиться, что

$$
\sup _{\substack{\lambda \in \Lambda, n \geqslant n_{\Lambda}}}\left\|\left(I-\lambda A_{n}^{-1}\right)^{-1}\right\|<\infty .
$$

Предположим противное, т.е. пусть $k_{n} \rightarrow \infty$ и $\lambda_{k_{n}} \in \Lambda$ - такая последовательность (можно предположить, что $\lambda_{k_{n}}$ сходится к $\bar{\lambda} \in \Lambda$ ), что

$$
\left\|\left(I-\lambda_{k_{n}} A_{k_{n}}^{-1}\right)^{-1}\right\| \rightarrow \infty .
$$

Поскольку $\lambda_{k_{n}} A_{h_{n}}^{-1}$ сходится компактно к $\bar{\lambda} A^{-1}$, это противоречит лемме 3.5.1.

Осталось доказать (3.5.3). Предполагая, что найдутся такие последовательности $k_{n} \rightarrow \infty$ и $\Lambda \ni \lambda_{k_{n}} \rightarrow \bar{\lambda} \in \Lambda$, и $\epsilon>0$, что

$$
\left\|\left(\lambda_{k_{n}} I_{k_{n}}-A_{k_{n}}\right)^{-1} p_{k_{n}} u-p_{k_{n}}\left(\lambda_{k_{n}} I-A\right)^{-1} u\right\| \geqslant \epsilon
$$

приходим к противоречию с тем фактом, что

$$
\left\|\left(\lambda I_{n}-A_{n}\right)^{-1} p_{n} u-p_{n}(\lambda I-A)^{-1} u\right\| \rightarrow 0 \quad \text { при } \lambda=\bar{\lambda}
$$

и оценкой (3.5.2).

Далее, покажем, что из компактной сходимости операторов $A_{n}^{-1}$ к $A^{-1}$ следует, что резольвентное множество оператора $A_{n}$ содержит некоторый фиксированный сектор при почти всех $n$. Введем обозначение

$$
\Sigma_{\phi, \bar{\omega}}=\{\lambda \in \mathbb{C}:|\arg (\lambda-\bar{\omega})|<\pi-\phi\} .
$$

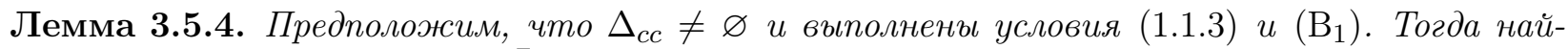
дутся такие сектор $\Sigma_{\bar{\phi},-\omega}, \phi<\bar{\phi}<\pi / 2,0<\omega<\tilde{\omega}$, и константы $n_{\omega}>0$ и $M_{\omega}$, что

$$
\left\|\left(\lambda I_{n}-A_{n}\right)^{-1}\right\| \leqslant \frac{M_{\omega}}{|\lambda-\omega|} \quad \text { для всех } \lambda \in \Sigma_{\bar{\phi},-\omega} u n \geqslant n_{\omega} .
$$

Доказательство. Пусть $\omega<\omega_{1}<\tilde{\omega}$. Рассмотрим область

$$
\Lambda=\left\{\lambda \in \mathbb{C}: \operatorname{Re} \lambda \geqslant \omega_{1}, \lambda \notin \Sigma_{\phi, \bar{\omega}}\right\} .
$$

Тогда $\Lambda$ - компактное множество и $\Lambda \subset \rho(A)$. Из леммы 3.5.3 следует, что найдется такая константа $n_{\Lambda}>0$, что $\Lambda \subset \rho\left(A_{n}\right)$ для всех $n \geqslant n_{\Lambda}$ и

$$
\sup _{\substack{\lambda \in \Lambda \\ n \geqslant n_{\Lambda}}}\left\|\left(\lambda I_{n}-A_{n}\right)^{-1}\right\|<\infty .
$$

Если выбрать $\bar{\phi}<\pi / 2$ так, что

$$
\Sigma_{\bar{\phi}, \omega} \cap\left\{\lambda \in \Sigma_{\phi, \tilde{\omega}}: \operatorname{Re} \lambda<\omega_{1}\right\}=\varnothing,
$$

получим, что $\Sigma_{\bar{\phi}, \omega} \subset \rho\left(A_{n}\right)$ для всех $n \geqslant n_{\Lambda}$. Оценка (3.5.5) следует из (3.5.6) и оценки $\left(\mathrm{B}_{1}\right)$.

Лемма 3.5.5. Пусть $A_{n}$ и А таковы, что выполнены условия (1.1.3), $\left(\mathrm{B}_{1}\right)$ и $\Delta_{c c} \neq \varnothing$. Тогда

$$
A_{n}^{-\theta} \stackrel{\mathcal{P P}}{\longrightarrow} A^{-\theta} \quad \text { компактно }
$$

для любого $0<\theta<1$.

Доказательство. Пусть сектор $\Sigma_{\bar{\phi},-\omega}$-такой же, как в лемме 3.5.4, и $\Omega$-его граница. Имеем

$$
\left(-A_{n}\right)^{-\theta}=\frac{1}{2 \pi i} \int_{\Omega}(-\lambda)^{-\theta}\left(\lambda I_{n}-A_{n}\right)^{-1} d \lambda .
$$


По лемме 3.5.4 указанный интеграл абсолютно и равномерно сходится при $n \geqslant n_{\omega}$. Следовательно, при заданном $\epsilon>0$ можно разбить на части контур интегрирования $\Omega=\Omega_{1}^{\epsilon} \cup \Omega_{2}^{\epsilon}$ таким образом, что $\Omega_{1}^{\epsilon}$ ограничена и

$$
\frac{1}{2 \pi i} \int_{\Omega_{2}^{\epsilon}}\left\|(-\lambda)^{-\theta}\left(\lambda I_{n}-A_{n}\right)^{-1}\right\| d \lambda \leqslant \epsilon, \quad n \geqslant n_{\omega} .
$$

Перепишем интеграл по $\Omega_{1}^{\epsilon}$ следующим образом:

$$
B_{n}:=\frac{A_{n}^{-1}}{2 \pi i} \int_{\Omega_{1}^{\epsilon}}(-\lambda)^{-\theta} \lambda\left(\lambda I_{n}-A_{n}\right)^{-1} d \lambda .
$$

Заметим, что величина $\left\|(-\lambda)^{-\theta} \lambda\left(\lambda I_{n}-A_{n}\right)^{-1}\right\|$ ограничена равномерно по $n \geqslant n_{\omega}$. Из того факта, что $A_{n}^{-1} \rightarrow A^{-1}$ компактно, следует, что $B_{n} \rightarrow B$ компактно. Пусть $\mu$-мера некомпактности. Выбирая произвольные последовательности $n \rightarrow \infty$ и $\left\{u_{n}\right\}, u_{n} \in E_{n},\left\|u_{n}\right\|=1$, получим

$$
\mu\left(\left\{\left(-A_{n}\right)^{-\theta} u_{n}\right\}\right) \leqslant \mu\left(B_{n}\left\{u_{n}\right\}\right)+\mu\left(\frac{1}{2 \pi i} \int_{\Omega_{2}^{\epsilon}}(-\lambda)^{-\theta}\left(\lambda I_{n}-A_{n}\right)^{-1} d \lambda u_{n}\right) \leqslant \epsilon .
$$

Итак, для данных последовательностей $n \rightarrow \infty$ и $\left\{u_{n}\right\}, u_{n} \in E_{n},\left\|u_{n}\right\|=1$, получаем, что $\mu\left(\left\{\left(-A_{n}\right)^{-\theta} u_{n}\right\}\right)=0$ и $\left(-A_{n}\right)^{-\theta} \rightarrow(-A)^{-\theta}$ компактно.

Для доказательства сходимости $\left(-A_{n}\right)^{-\theta} \stackrel{\mathcal{P P}}{\longrightarrow}(-A)^{-\theta}$ воспользуемся тем фактом, что $\left(\lambda I_{n}-A_{n}\right)^{-1} \stackrel{\mathcal{P P}}{\longrightarrow}(\lambda I-A)^{-1}$ для всех $\lambda \in \Omega$ при $n \rightarrow \infty$, и применим теорему о мажорантной сходимости.

Теперь рассмотрим относительно ограниченные возмущения операторов $A_{n}$ и $A$. Предположим, что возмущения удовлетворяют следующим условиям:

(L) $D_{n} \in B\left(E_{n}^{\theta}, E_{n}\right)$ и $\left.D \in B\left(E^{\theta}, E\right)\right)$, так что $D_{n}$ и $D$ согласованы.

Наложим также следующее условие гиперболичности:

(H) $\sigma(A+D) \cap\{\lambda \in \mathbb{C}: \operatorname{Re} \lambda=0\}=\varnothing$.

Ясно, что если резольвента оператора $A$ компактна, то оператор $A+D$ имеет компактную резольвенту.

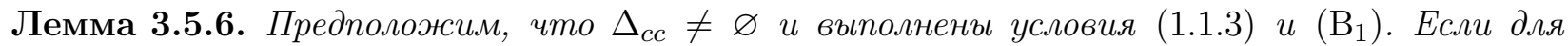
любого $0 \leqslant \xi<1$ операторы $D_{n} \in B\left(E_{n}^{\theta}, E_{n}\right)$ таковы, что $D_{n} \rightarrow D$ в смысле пространств $E_{n}^{\theta}$, $E_{n} u E^{\theta}, E, m o$

$$
\left(-A_{n}\right)^{\xi}\left(A_{n}+D_{n}\right)^{-1} \stackrel{\mathcal{P P}}{\longrightarrow}(-A)^{\xi}(A+D)^{-1} \quad \text { компактно. }
$$

Доказательство. Выберем положительные числа $r_{1}$ и $r_{2}$ так, чтобы $1>\theta+r_{1} \geqslant \xi$ и $r_{2}=1-\theta-r_{1}$, и заметим, что

$$
\left(-A_{n}\right)^{\xi}\left(A_{n}+D_{n}\right)^{-1}=\left(-A_{n}\right)^{-\left(\theta+r_{1}-\xi\right)}\left(-I_{n}+\left(-A_{n}\right)^{-r_{2}} D_{n}\left(-A_{n}\right)^{-\theta-r_{1}}\right)^{-1}\left(-A_{n}\right)^{-r_{2}} .
$$

Поскольку $\left\{\left(-A_{n}\right)^{-r_{2}} D_{n}\left(-A_{n}\right)^{-\theta-r_{1}}\right\}$ сходится компактно, требуемый результат вытекает из лемм 3.5.5 и 3.5.1.

Лемма 3.5.7. Пусть $A_{n}$ и $A$ таковы, что выполнены условия (1.1.3), $\left(\mathrm{B}_{1}\right), \Delta_{c c} \neq \varnothing,(\mathrm{L})$ $u(\mathrm{H})$. Тогда найдутся такие сектор $\Sigma_{\phi_{d}, \omega_{d}}, \phi_{d}<\pi / 2, \omega_{d} \in \mathbb{R}$, и константы $n_{d}>0$ и $M_{d}$, что

$$
\left\|\left(\lambda I_{n}-\left(A_{n}+f_{n}^{\prime}\left(u_{n}^{*}\right)\right)\right)^{-1}\right\| \leqslant \frac{M_{d}}{\left|\lambda-\omega_{d}\right|} \quad \text { для всех } \lambda \in \Sigma_{\phi_{d}, \omega_{d}} \text { u всех } n \geqslant n_{d} .
$$

Доказательство. Из леммы 3.5.4 и неравенства моментов следует, что

$$
\left\|\left(-A_{n}\right)^{\theta}\left(\lambda I_{n}-A_{n}\right)^{-1}\right\| \leqslant \frac{M}{|\lambda|^{1-\theta}}, \quad n \geqslant n_{\omega},
$$




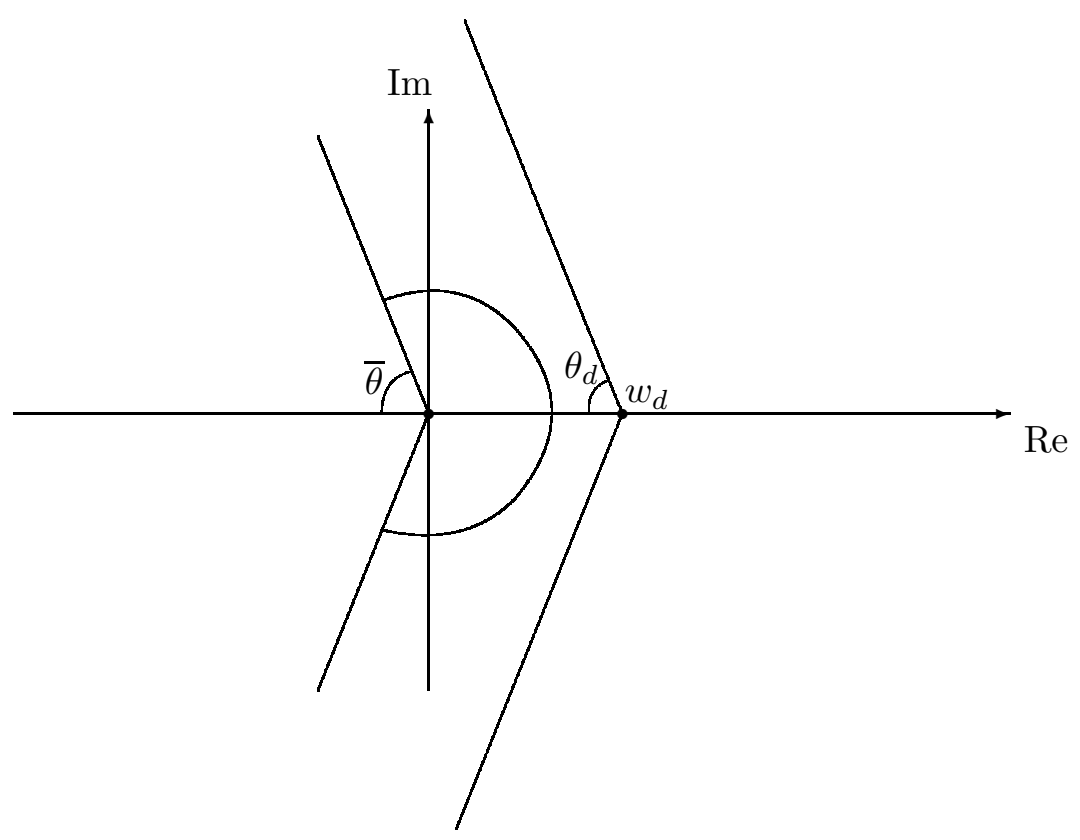

Рис. 3

для $\lambda \in \Sigma_{\bar{\phi}, 0}$. Поскольку оператор $D_{n}\left(-A_{n}\right)^{-\theta}$ ограничен равномерно по $n$ и

$$
\left(\lambda I_{n}-A_{n}-D_{n}\right)=\left(I_{n}-D_{n}\left(-A_{n}\right)^{-\theta}\left(-A_{n}\right)^{\theta}\left(\lambda I_{n}-A_{n}\right)^{-1}\right)\left(\lambda I_{n}-A_{n}\right),
$$

найдется такое $R>0$, что при $|\lambda|>R, \lambda \in \Sigma_{\bar{\phi}, 0}$, оператор $\left(I_{n}-D_{n}\left(-A_{n}\right)^{-\theta}\left(-A_{n}\right)^{\theta}\left(\lambda I_{n}-A_{n}\right)\right)$ обратим и

$$
\left\|\left(I_{n}-D_{n}\left(-A_{n}\right)^{-\theta}\left(-A_{n}\right)^{\theta}\left(\lambda I_{n}-A_{n}\right)^{-1}\right)^{-1}\right\| \leqslant 2 .
$$

Отсюда получаем требуемый результат (см. рис. 3) для любого $\omega_{d}>R$ и надлежащим образом выбранного $\theta_{d}: \Sigma_{\theta_{d}, \omega_{d}} \subset\left\{\lambda \in \Sigma_{\bar{\theta}, 0}:|\lambda|>R\right\}$.

Замечая, что, согласно лемме 3.5.6, $\left(A_{n}+f_{n}^{\prime}\left(u_{n}^{*}\right)\right)^{-1}$ сходится компактно к $\left(A+f^{\prime}\left(u^{*}\right)\right)^{-1}$, и рассуждая, как в доказательстве леммы 3.5.3, получим следующий результат.

Лемма 3.5.8. Пусть $A_{n}$ и A таковы, что выполнены условия (1.1.3), $\left(\mathrm{B}_{1}\right), \Delta_{c c} \neq \varnothing,(\mathrm{L})$, $u(\mathrm{H})$. Пусть $\Lambda-$ компактное подмножество множества $\rho\left(\left(A+f^{\prime}\left(u^{*}\right)\right)\right)$. Тогда найдется такая константа $n_{\Lambda}>0$, что $\Lambda \subset \rho\left(\left(A_{n}+f_{n}^{\prime}\left(u_{n}^{*}\right)\right)\right)$ для всех $n \geqslant n_{\Lambda} u$

$$
\sup _{\substack{\lambda \in \Lambda \\ n \geqslant n_{\Lambda}}}\left\|\left(\lambda I_{n}-\left(A_{n}+f_{n}^{\prime}\left(u_{n}^{*}\right)\right)\right)^{-1}\right\|<\infty
$$

Кроме того,

$$
\sup _{\lambda \in \Lambda}\left\|\left(\lambda I_{n}-\left(A_{n}+f_{n}^{\prime}\left(u_{n}^{*}\right)\right)\right)^{-1} p_{n} u-\left(\lambda I-\left(A+f^{\prime}\left(u^{*}\right)\right)\right)^{-1} u\right\| \rightarrow 0
$$

при $n \rightarrow \infty$ для любого $u \in E$.

С каждой изолированной точкой $\lambda \in \sigma\left(\left(A+f^{\prime}\left(u^{*}\right)\right)\right)$ свяжем корневое подпространство $W\left(\lambda,\left(A+f^{\prime}\left(u^{*}\right)\right)\right)=P\left(\lambda,\left(A+f^{\prime}\left(u^{*}\right)\right)\right) E$, где

$$
P\left(\lambda,\left(A+f^{\prime}\left(u^{*}\right)\right)\right)=\frac{1}{2 \pi i} \int_{|\xi-\lambda|=\delta}\left(\xi I-\left(A+f^{\prime}\left(u^{*}\right)\right)\right)^{-1} d \xi
$$


и $\delta$ настолько мало, что в круге $\{\xi \in \mathbb{C}:|\xi-\lambda| \leqslant \delta\}$ нет точек множества $\sigma\left(\left(A+f^{\prime}\left(u^{*}\right)\right)\right)$. Введем обозначение

где

$$
W_{n}\left(\lambda,\left(A_{n}+f_{n}^{\prime}\left(u_{n}^{*}\right)\right)\right)=P_{n}\left(\lambda,\left(A_{n}+f_{n}^{\prime}\left(u_{n}^{*}\right)\right)\right) E_{n},
$$

$$
P_{n}\left(\lambda,\left(A_{n}+f_{n}^{\prime}\left(u_{n}^{*}\right)\right)\right)=\frac{1}{2 \pi i} \int_{|\xi-\lambda|=\delta}\left(\xi I_{n}-\left(A_{n}+f_{n}^{\prime}\left(u_{n}^{*}\right)\right)\right)^{-1} d \xi
$$

Теорема 3.5.1. Пусть $A_{n}$ и А таковы, что выполнены условия (1.1.3), $\left(\mathrm{B}_{1}\right), \Delta_{c c} \neq \varnothing,(\mathrm{L})$ и (Н) . Тогда справедливы следующие утверждения:

(i) существует такое $n_{0}>0$, что

$$
\operatorname{dim} W_{n}\left(\sigma_{n}^{+},\left(A_{n}+f_{n}^{\prime}\left(u_{n}^{*}\right)\right)\right)=\operatorname{dim} W\left(\sigma^{+},\left(A+f^{\prime}\left(u^{*}\right)\right)\right)
$$

для всех $n \geqslant n_{0}$

(ii) для любого $u \in W\left(\sigma^{+},\left(A+f^{\prime}\left(u^{*}\right)\right)\right)$ существует такая последовательность $\left\{u_{n}\right\}$, $u_{n} \in W_{n}\left(\sigma^{+},\left(A_{n}+f_{n}^{\prime}\left(u_{n}^{*}\right)\right)\right)$, чmo $u_{n} \stackrel{\mathcal{P}^{\theta}}{\longrightarrow} u$;

(iii) любал последовательность $\left\{u_{n}\right\}, u_{n} \in W_{n}\left(\sigma_{n}^{+},\left(A_{n}+f_{n}^{\prime}\left(u_{n}^{*}\right)\right)\right), n \in \mathbb{N},\left\|u_{n}\right\|_{X_{n}}=1$, имеет сходящуюся подпоследовательность, причем любая предельная точка этой последовательности принадлежит множеству $W\left(\sigma^{+},\left(A+f^{\prime}\left(u^{*}\right)\right)\right)$;

(iv) существуют такие $\beta>0$ и $n_{\beta}>0$, что

$$
\sigma\left(\left(A_{n}+f_{n}^{\prime}\left(u_{n}^{*}\right)\right)\right) \cap\{\lambda \in \mathbb{C}:|\operatorname{Re} \lambda|>\beta\}=\varnothing, \quad n \geqslant n_{\beta} ;
$$

(v) найдутся такие $\pi / 2>\phi_{\beta}>0, \omega_{\beta}>0$ u $n_{\beta}>0$, что $\Sigma_{\phi_{\beta},-\omega_{\beta}} \subset \rho\left(A_{n}\right), n \geqslant n_{\beta}, u$

$$
\left\|\left(\lambda I_{n}-\left(A_{n}+f_{n}^{\prime}\left(u_{n}^{*}\right)\right)\right)^{-1}\right\| \leqslant \frac{M_{\beta}}{\left|\lambda-\omega_{\beta}\right|} \quad \text { для всех } \lambda \in \Sigma_{\phi_{\beta},-\omega_{\beta}} \text { и всех } n \geqslant n_{\beta} .
$$

3.5.1. Сходимость линейных полугрупп и равномерные оценки. Хорошо известно, что если выполнено условие (1.1.3), то для любого $\omega<\tilde{\omega}$ найдется такая константа $M_{\omega} \geqslant 1$, что

$$
\left\|t^{\theta} e^{A t}\right\|_{B\left(E, E^{\theta}\right)} \leqslant M_{\omega} e^{-\omega t} \quad \forall t \geqslant 0 .
$$

Из леммы 3.5.4 следует, что для полугрупп, порожденных операторами $A_{n}$, при достаточно больших $n$ имеет место равномерная оценка, аналогичная (1.1.3). Таким образом, получаем следующий результат.

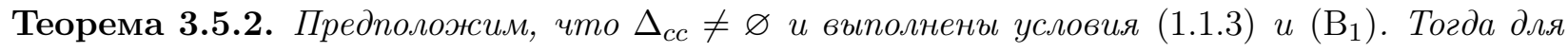
любого $\omega<\tilde{\omega}$ существуют такие константы $n_{0}$ и $M_{\omega}$, что

$$
\left\|t^{\theta} e^{A_{n} t}\right\|_{B\left(E_{n}, E_{n}^{\theta}\right)} \leqslant M_{\omega} e^{-\omega t} \quad \forall t \geqslant 0, \quad 0 \leqslant \theta \leqslant 1, \quad n \geqslant n_{0} .
$$

Доказательство. Рассмотрим сектор

$$
\Sigma(\pi-\phi, \omega)=\{\lambda \in \mathbb{C}:|\arg (\lambda-\omega)|<\pi-\phi\}, \quad \omega \in \mathbb{R}, \quad 0<\phi<\frac{\pi}{2} .
$$

Из условия $\left(\mathrm{B}_{1}\right)$ получим такие подходящие значения $\omega_{3} \in \mathbb{R}, n_{\omega} \in \mathbb{N}$, и $0<\phi<\pi / 2$, чтобы

$$
\left\|\left(\lambda I_{n}-A_{n}\right)^{-1}\right\| \leqslant \frac{M_{3}}{\left|\lambda-\omega_{3}\right|} \text { для всех } \lambda \in \Sigma\left(\pi-\phi, \omega_{3}\right) \text { и } n \geqslant n_{\omega} .
$$

Из (1.1.3) следует, что $\operatorname{Re} \sigma(A)<\omega^{\prime}$ для некоторого $\omega^{\prime}<0$ и, кроме того,

для $\operatorname{Re} \lambda>\omega^{\prime}$.

$$
\left\|(\lambda I-A)^{-1}\right\| \leqslant \frac{M}{\left|\lambda-\omega^{\prime}\right|}
$$

Пусть теперь $0<\tilde{\omega}<\left|\omega^{\prime}\right|$; рассмотрим треугольную область (см. рис. 4)

$$
\Lambda=\left\{\lambda \in \mathbb{C}: \operatorname{Re} \lambda \geqslant-\tilde{\omega}, \lambda \notin \Sigma\left(\pi-\phi, \omega_{3}\right)\right\} .
$$

Из теоремы 3.5.1 вытекает следующее утверждение. 


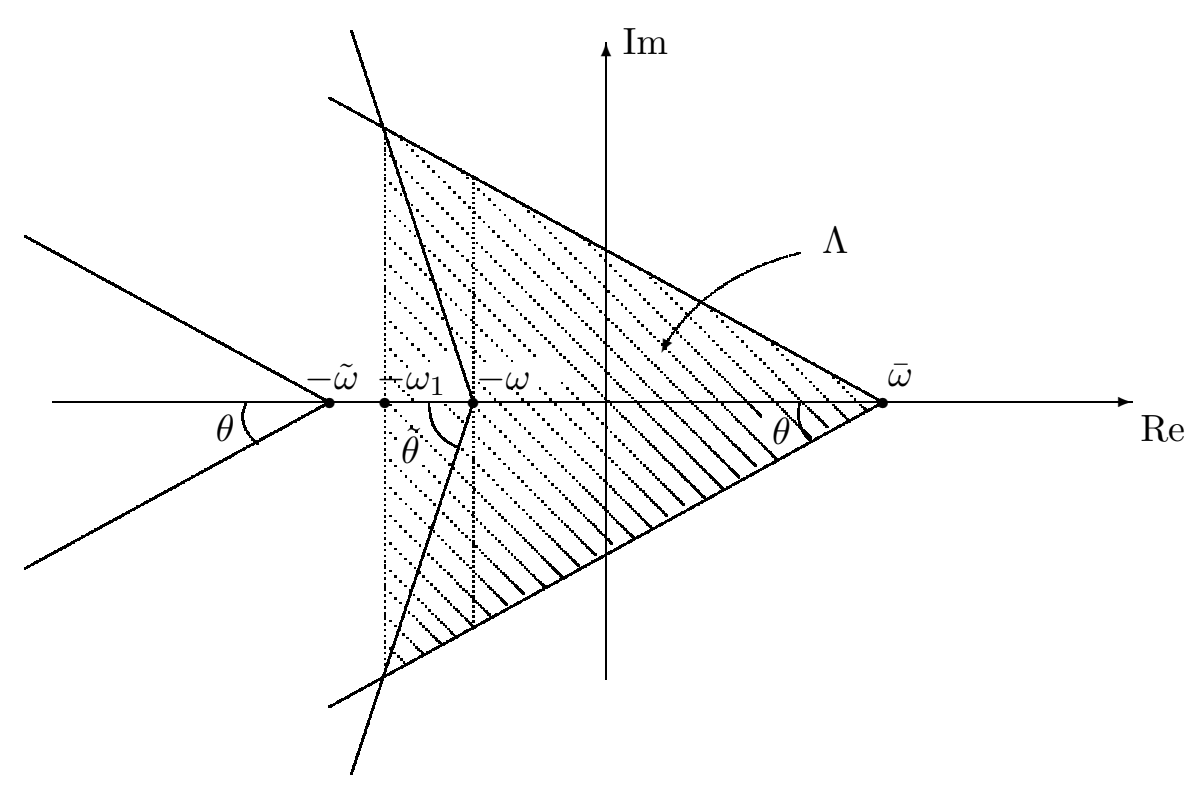

Рис. 4

Теорема 3.5.3. Предположим, что выполнены условие $\Delta_{c c} \neq \varnothing$ и условия (1.1.3), $\left(\mathrm{B}_{1}\right),(\mathrm{L})$ $u(\mathrm{H})$. Тогда для любого $\omega_{\beta}<\beta$ найдутся такие константы $n_{\omega_{\beta}}$ u $M_{\omega_{\beta}}$, что при $n \geqslant n_{\omega_{\beta}}$ выполнены следующие неравенства:

$$
\begin{array}{ll}
\left\|t^{\theta} e^{\left(A_{n}+f_{n}^{\prime}\left(u_{n}^{*}\right)\right) t}\left(I_{n}-P_{n}\left(\sigma_{n}^{+},\left(A_{n}+f_{n}^{\prime}\left(u_{n}^{*}\right)\right)\right)\right)\right\|_{B\left(E_{n}, E_{n}^{\theta}\right)} \leqslant M_{\omega_{\beta}} e^{-\omega_{\beta} t} & \forall t \geqslant 0, \quad 0 \leqslant \theta \leqslant 1, \\
\left\|e^{\left(A_{n}+f_{n}^{\prime}\left(u_{n}^{*}\right)\right) t} P_{n}\left(\sigma_{n}^{+},\left(A_{n}+f_{n}^{\prime}\left(u_{n}^{*}\right)\right)\right)\right\|_{B\left(E_{n}\right)} \leqslant M_{\omega_{\beta}} e^{\omega_{\beta} t} & \forall t \leqslant 0 .
\end{array}
$$

Замечание 3.5.1. Дискретная сходимость резольвент (в отсутствие условия $\Delta_{c c} \neq \varnothing$ ) не является достаточным условием для теоремы 3.5.3, что подтверждает следующим пример.

Для любого $n \geqslant 1$, пусть $L_{n}-(n \times n)$-матрица, все элементы которой равны нулю, за исключением элементов первой наддиагонали, которые равны 1 . Пусть $M_{n}=-\omega I_{n}+L_{n}$. Единственной точкой спектра матрицы $M_{n}$ является число $-\omega$. Кроме того, поскольку

$$
\left\|\exp \left(t M_{n}\right)\right\| \leqslant e^{-\omega t} e^{\left\|L_{n}\right\| t} \leqslant e^{(1-\omega) t},
$$

ясно, что на конечных интервалах имеет место сильная сходимость к $e^{-\alpha t} I$ полугрупп, порожденных операторами

$$
A_{n}=\operatorname{diag}\left(-\alpha I_{1},-\alpha I_{2}, \ldots, M_{n}, 0,0 \ldots\right) .
$$

$\mathrm{C}$ другой стороны, $(1, n)$-элемент матрицы $\exp \left(t M_{n}\right)$ равен

$$
e^{-\omega t} \frac{t^{n-1}}{(n-1) !}
$$

по формуле Стирлинга

$$
\left\|e^{(n-1) M_{n}}\right\| \geqslant e^{-\omega(n-1)} \frac{(n-1)^{n-1}}{(n-1) !} \equiv \frac{e^{(n-1)(1-\omega)}}{(2 \pi n)^{1 / 2}},
$$

и для $0<\omega<1$ имеем

$$
\sup \left\{\left\|e^{(n-1) M_{n}}\right\|: n \geqslant 1, t \geqslant 0\right\}=+\infty
$$




\section{ГЛАВА 4}

\section{ЗАТЕНЕНИЕ ДЛЯ АБСТРАКТНЫХ ПАРАБОЛИЧЕСКИХ УРАВНЕНИЙ}

Классические результаты о затенении состоят в том, что псевдотраектории конечномерных динамических систем могут «затеняться» настоящими траекториями при условии, что система обладает некоторым типом гиперболичности. Обычно это формулируется следующим образом: для любого данного $\varepsilon>0$ существует такое $\delta>0$, что для любой псевдотраектории, имеющей скачки величины $\delta$ в последовательные моменты времени, в $\varepsilon$-окрестности этой псевдотраектории равномерно по времени существует истинная траектория. Такие утверждения справедливы как для непрерывных, так и для дискретных по времени динамических систем в окрестностях (надлежащим образом определенных) гиперболических множеств. Мы отсылаем читателя к работам $[116,213,218]$, содержащим прекрасные изложения различных результатов о затенении.

Отметим, что в русскоязычной литературе еще не появилось общепринятого эквивалента английского термина shadowing; чаще всего встречается термин «отслеживание» («свойство отслеживания»), хотя в переводе известной монографии [18] А. Б. Катка и Б. Хасселблата используются термины «приближение псевдоорбит» (shadowing of pseudo-orbits), «лемма о $\varepsilon$-траекториях» (shadowing lemma). В настоящей работе будем использовать кальку английского термина - затенение.

Когда в такой подход включаются вопросы численных аппроксимаций, становится понятно, что концепция псевдотраектории нуждается в значительном расширении, чтобы принцип затенения оставался в силе. Например, дискретизация автономного обыкновенного дифференциального уравнения одношаговым методом приводит к отображению, зависящему от величины шага, и затенение теперь означает аппроксимацию дискретной временной орбиты истинной непрерывной траекторией или наоборот. Результат такого типа был получен в [77] вблизи стационарных гиперболических точек, а для более общих гиперболических ситуаций - в [115].

Результаты о затенении для численных аппроксимаций зависящих от времени уравнений с частными производными обычно включают дискретизацию как по времени, так и по пространству, т.е. непрерывная траектория в бесконечномерном пространстве должна быть затенена дискретной по времени траекторией в конечномерном пространстве и наоборот. Такой результат был получен в [168] для метода конечных элементов в сочетании с обратной дискретизацией Эйлера по времени при применении к нелинейной системе «реакция-диффузия» в окрестности стационарного гиперболического решения. Эти результаты обобщили ранние работы тех же авторов по полудискретизации с конечными элементами (см. [167]). Результаты по затенению для полудискретизации по времени вблизи гиперболических стационарных состояний были получены ранее в [38], а для линейной, но неавтономной ситуации - сравнительно недавно в [208].

Цель этой главы - изучить свойства затенения довольно общих пространственных дискретизаций нелинейного эволюционного уравнения (1.2.16), где $A$-замкнутый оператор, порождающий аналитическую $C_{0}$-полугруппу $\exp (t A)$ на $E$. Для дискретизации в пространстве используем теорию дискретных приближений, разработанную в $[121,244,256,259,261]$. Как известно, для стационарных задач эта теория обеспечивает единую основу для обработки таких разнообразных аппроксимаций, как (конформные и неконформные) методы конечных элементов, методы конечных разностей (см. [256]) и возмущения областей (см. [51,245]).

Основные результаты этой главы (см. теоремы 4.3.1 и 4.3.2) утверждают, что обобщенные решения вблизи гиперболического равновесия системы (4.1.5) могут быть затенены на произвольных больших интервалах времени соответствующими обобщенными решениями системы (4.2.9) и наоборот. Наш подход здесь следует общей идее построения затеняющих траекторий из краевых задач, как в $[38,77,167,168]$. 


\section{1. ПРЕДВАРИТЕЛЬНЫЕ СВЕДЕНИЯ}

Рассмотрим в банаховом пространстве $E$ следующую неоднородную задачу Коши:

$$
\begin{aligned}
& u^{\prime}(t)=A u(t)+g(t), \quad t \in[0, T], \\
& u(0)=u_{0},
\end{aligned}
$$

где оператор $A \in \mathcal{C}(E)$ порождает $C_{0}$-полугруппу и $g(\cdot)$ - функция $[0, T] \rightarrow E$. Задачу (4.1.1) можно рассматривать в различных функциональных пространствах. Наиболее популярные пространства, для которых легко доказывается корректность задачи, - это $C([0, T] ; E), C_{0}^{\alpha}([0, T] ; E)$ и $L^{p}([0, T] ; E)($ см. $[60,262])$.

Рассмотрим так называемое обобщенное решение задачи (4.1.1), т.е. функцию

$$
u(t)=\exp (t A) u^{0}+\int_{0}^{t} \exp ((t-s) A) g(s) d s, \quad t \geqslant 0 .
$$

В этой главе, так же, как в главе 3, будем изучать полулинейную автономную абстрактную параболическую задачу

$$
\begin{aligned}
& u^{\prime}(t)=A u(t)+f(u(t)), \quad t \geqslant 0, \\
& u(0)=u^{0} \in E,
\end{aligned}
$$

где функция $f(\cdot): E \rightarrow E$ локально липшицева, ограничена и непрерывно дифференцируема по Фреше. Хорошо известно, что в этих предположениях обобщенное решение уравнения (4.1.3) определено на максимальном интервале. Кроме того, записав решение при $u(t)=S(t) u^{0}$, получим нелинейную полугруппу $S(\cdot)$ на $E$, что удовлетворяет формуле вариации постоянной

$$
S(t) u^{0}=\exp (t A) u^{0}+\int_{0}^{t} e^{(t-s) A} f\left(S(s) u^{0}\right) d s, \quad t \geqslant 0 .
$$

В дальнейшем будем предполагать, что $A: D(A) \subseteq E \rightarrow E$-замкнутый линейный оператор, для которого имеет место (1.1.3).

Для некоторого $0<\theta \leqslant 1$ рассмотрим следующее полулинейное уравнение (4.1.3) в пространстве $E^{\theta}$ :

$$
\begin{aligned}
& u^{\prime}(t)=A u(t)+f(u(t)), \quad t \geqslant 0, \\
& u(0)=u^{0} \in E^{\theta}
\end{aligned}
$$

здесь $f(\cdot): E^{\theta} \subseteq E \rightarrow E$ удовлетворяет следующему условию:

(F1) для некоторого $\rho>0$ функция $f(\cdot): \mathcal{U}_{E^{\theta}}\left(u^{*} ; \rho\right) \mapsto E$ непрерывно дифференцируемо по Фреше и для любого $\epsilon>0$ найдется такое $\delta>0$, что

$$
\left\|f^{\prime}(w)-f^{\prime}(z)\right\|_{B\left(E^{\theta}, E\right)} \leqslant \epsilon
$$

для всех $w, z \in \mathcal{U}_{E^{\theta}}\left(u^{*} ; \rho\right)$, удовлетворяющих условию $\|w-z\|_{E^{\theta}} \leqslant \delta$, где $u^{*}$ - гиперболическая точка равновесия уравнения (4.1.5).

В дальнейшем всегда будем обозначать через $u^{*}$ гиперболическое равновесие задачи (4.1.3). Аналогично (3.1.10), заменой переменных $v(\cdot)=u(\cdot)-u^{*}$ можно привести задачу (4.1.5) к виду

$$
v^{\prime}(t)=A_{u^{*}} v(t)+F_{u^{*}}(v(t)), \quad v(0)=v^{0}, \quad t \geqslant 0,
$$

где $v^{0}=u^{0}-u^{*}$ и

$$
A_{u^{*}}=A+f^{\prime}\left(u^{*}\right), \quad F_{u^{*}}(w)=f\left(w+u^{*}\right)-f\left(u^{*}\right)-f^{\prime}\left(u^{*}\right) w \text { при }\|w\|_{E^{\theta}} \leqslant \rho .
$$

Напомним, что $F_{u^{*}}(w)=f\left(w+u^{*}\right)-f\left(u^{*}\right)-f^{\prime}\left(u^{*}\right) w$ имеет порядок $o\left(\|w\|_{E^{\theta}}\right)$, а оператор $A_{u^{*}}$ порождает аналитическую $C_{0}$-полугруппу, поскольку $f^{\prime}\left(u^{*}\right) \in B\left(E^{\theta}, E\right)$ (см. [26]). 
Предположим, что часть $\sigma^{+}$спектра оператора $A+f^{\prime}\left(u^{*}\right)$, расположенная строго правее мнимой оси, состоит из конечного числа собственных значений конечной кратности. Это предположение выполнено, например, если резольвента оператора $A$ компактна. В случае гиперболической точки $u^{*}$ на мнимой оси $i \mathbb{R}$ нет точек спектра оператора $A_{u^{*}}$.

Пусть $U\left(\sigma^{+}\right) \subset\{\lambda \in \mathbb{C}: \operatorname{Re} \lambda>0\}$ - открытая связная окрестность множества $\sigma^{+}$, граница которой $\partial U\left(\sigma^{+}\right)$является замкнутой спрямляемой кривой. Разложим пространство $E^{\theta}$, используя проектор Рисса, определенный множеством $\sigma^{+}$, как в (3.1.8). Согласно определению и аналитичности $C_{0}$-полугруппы $\exp \left(t A_{u^{*}}\right)$ имеем такие положительные константы $M_{1}, \beta>0$, что (см. [136])

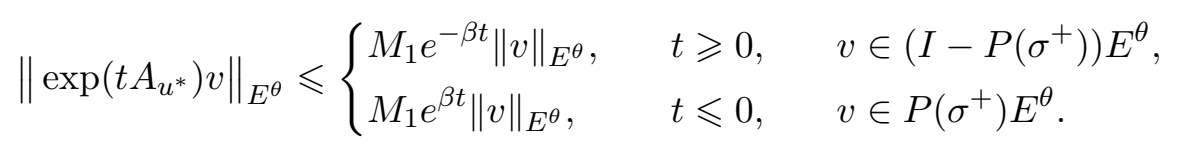

Без ограничения общности можно выбрать норму в $E^{\theta}$ следующим образом:

$$
\|v\|_{E^{\theta}}=\max \left(\|P(\sigma+) v\|_{E^{\theta}},\|(I-P(\sigma+)) v\|_{E^{\theta}}\right) .
$$

Если элемент $v^{0}$ близок к 0 , например, $v^{0} \in \mathcal{U}_{E^{\theta}}(0 ; \rho)$, то обобщенное решение $v\left(t ; v^{0}\right)$ задачи (4.1.6) остается внутри шара $\mathcal{U}_{E^{\theta}}(0 ; \rho)$ в течение некоторого времени. Мы будем считать такое решение решением краевой задачи, в которой устойчивая часть задана в начале траектории, а неустойчивая - в конце траектории. Точнее, для любых двух $v^{-}, v^{+} \in \mathcal{U}_{E^{\theta}}(0 ; \rho)$ и для любого $0<T \leqslant \infty$ рассмотрим краевую задачу

$$
\begin{cases}v^{\prime}(t)=A_{u^{*}} v(t)+F_{u^{*}}(v(t)), & 0 \leqslant t \leqslant T, \\ \left(I-P\left(\sigma^{+}\right)\right) v(0)=\left(I-P\left(\sigma^{+}\right)\right) v^{-}, & P\left(\sigma^{+}\right) v(T)=P\left(\sigma^{+}\right) v^{+} .\end{cases}
$$

В случае $T=\infty$ второе граничное условие отсутствует, и дифференциальное уравнение рассматривается на $[0, \infty)$. Обобщенное решение задачи (4.1.10) удовлетворяет интегральному уравнению

$$
\begin{aligned}
v(t)=\exp \left((t-T) A_{u^{*}}\right) P\left(\sigma^{+}\right) v^{+}+\exp \left(t A_{u^{*}}\right)(I- & \left.P\left(\sigma^{+}\right)\right) v^{-}+ \\
& +\int_{0}^{T} \Gamma_{T}(t, s) F_{u^{*}}(v(s)) d s, \quad 0 \leqslant t \leqslant T,
\end{aligned}
$$

где функция Грина $\Gamma_{T}(\cdot, \cdot)$ определена формулой

$$
\Gamma_{T}(t, s)= \begin{cases}\exp \left((t-s) A_{u^{*}}\right)\left(I-P\left(\sigma^{+}\right)\right), & 0 \leqslant s \leqslant t \leqslant T \\ \exp \left((t-s) A_{u^{*}}\right) P\left(\sigma^{+}\right), & 0 \leqslant t<s \leqslant T .\end{cases}
$$

Отметим, что из (4.1.8) следует

$$
\left\|\Gamma_{T}(t, s) z\right\|_{E^{\theta}} \leqslant M_{1} \frac{e^{-\beta|t-s|}}{|t-s|^{\alpha}}\|z\|_{E}
$$

В случае $T=\infty$ положим слагаемое в (4.1.11), включающее $v^{+}$, равным нулю. Существование и единственность решений задачи (4.1.11) устанавливается следующим предложением.

Предложение 4.1 .1 (см. [79]). Пусть $A$ и $f(\cdot)$ удовлетворяют сформулированным выше условиям, в частности, условию (F1). Тогда существует такое $\hat{\rho}>0$, что для любого $0<\hat{\rho}_{2} \leqslant \hat{\rho}$ найдется $0<\hat{\rho}_{1} \leqslant \hat{\rho}_{2}$, обладающее тем свойством, что уравнение (4.1.11) имеет единственное решение $v(\cdot)=v\left(u^{+}, u^{-}, \cdot\right) \in C\left([0, T] ; \mathcal{U}_{E^{\theta}}\left(0 ; \hat{\rho}_{2}\right)\right)$ для всех $v^{ \pm} \in \mathcal{U}_{E^{\alpha}}\left(0, \hat{\rho}_{1}\right)$ и всех $0<T \leqslant \infty$. Если $T=\infty$, mo $\|v(t)\|_{E^{\theta}} \rightarrow 0$ npu $t \rightarrow \infty$.

Доказательство. Применим лемму 4.1.1, полагая $Y=Z=C\left([0, T] ; E^{\theta}\right)$ (в случае $T=$ $\infty$ имеется в виду пространство непрерывных ограниченных функций). Положим $y_{0}=0$, $H(v)=v-G\left(u^{-}, u^{+} ; v\right)$, где оператор $G\left(v^{-}, v^{+} ; v\right)$ определен правой частью (4.1.11). Во-первых, 
заметим, что $H^{\prime}(0)=I-G_{v}^{\prime}\left(u^{-}, u^{+} ; 0\right)=I$, так что в лемме 4.1.1 можно взять $\sigma=1$. Для любых двух $v, w \in \mathcal{U}_{Y}\left(0, \hat{\rho}_{2}\right)$ имеем согласно (4.1.13) и (F1)

$$
\begin{aligned}
& \left\|\left(G_{v}^{\prime}\left(v^{-}, v^{+} ; v\right)-G_{v}^{\prime}\left(v^{-}, v^{+} ; w\right)\right) u\right\|_{Z} \leqslant \\
& \leqslant \sup _{0 \leqslant t \leqslant T}\left\|\int_{0}^{T} \Gamma_{T}(t, s)\left(F_{u^{*}, v}^{\prime}(v(s))-F_{u^{*}, v}^{\prime}(w(s))\right) u(s) d s\right\|_{E^{\theta}} \leqslant \\
& \leqslant M_{1} \sup _{0 \leqslant t \leqslant T} \int_{0}^{T} \frac{e^{-\beta|t-s|}}{|t-s|^{\theta}}\left\|\left(f^{\prime}\left(v(s)+u^{*}\right)-f^{\prime}\left(w(s)+u^{*}\right)\right) u(s)\right\|_{E} d s \leqslant \\
& \leqslant M_{1} \frac{1}{\beta}\|u\|_{Y} \sup _{v_{1}, v_{2} \in \mathcal{U}_{E^{\theta}}\left(0, \hat{\rho}_{2}\right)}\left\|f^{\prime}\left(v_{1}\right)-f^{\prime}\left(v_{2}\right)\right\|_{B\left(E^{\theta} ; E\right)} \leqslant \frac{1}{2}\|u\|_{Y}
\end{aligned}
$$

для достаточно малых $\hat{\rho}_{2}$. Наконец, выбрав $\hat{\rho}_{1}=\hat{\rho}_{2} /\left(4 M_{1}\right)$, получим

$$
\|H(0)\|_{E^{\theta}} \leqslant \sup _{0 \leqslant t \leqslant T}\left(M_{1} e^{-\beta t}\left\|\left(I-P\left(\sigma^{+}\right)\right) u^{-}\right\|_{E^{\theta}}+M_{1} e^{\beta(t-T)}\left\|P\left(\sigma^{+}\right) u^{+}\right\|_{E^{\theta}}\right) \leqslant M_{1} 2 \hat{\rho}_{1} \leqslant \frac{1}{2} \hat{\rho}_{2} .
$$

Теперь рассмотрим случай $T=\infty$, записав (4.1.11) в виде

$$
\begin{aligned}
v(t) & =\exp \left(t A_{u^{*}}\right)\left(I-P\left(\sigma^{*}\right)\right) v(0)+ \\
& +\int_{0}^{t} \exp \left((t-s) A_{u^{*}}\right)\left(I-P\left(\sigma^{+}\right)\right) F_{u^{*}}(v(s)) d s+\int_{t}^{\infty} \exp \left((t-s) A_{u^{*}}\right) P\left(\sigma^{+}\right) F_{u^{*}}(v(s)) d s .
\end{aligned}
$$

Будем рассуждать, как и выше, заменив пространство $C\left([0, \infty) ; E^{\theta}\right)$ на

$$
C_{0}\left([0, \infty) ; E^{\theta}\right)=\left\{u(\cdot) \in C\left([0, \infty) ; E^{\theta}\right):\|u(t)\|_{E^{\theta}} \rightarrow 0 \text { при } t \rightarrow \infty\right\} .
$$

Заметим, что $G\left(u^{-}, u^{+}, \cdot\right)$ отображает это пространство в себя, поскольку из того факта, что $\|v(t)\|_{E^{\theta}} \rightarrow 0$ при $t \rightarrow \infty$, следует

$$
\left\|F_{u^{*}}(v(t))\right\|_{E} \rightarrow 0 \quad \text { при } t \rightarrow \infty .
$$

Оператор $G\left(v^{-} ; v\right)$, определенный правой частью (4.1.15), непрерывен по обоим аргументам и отображает пространство $\left.C_{0}\left([0 ; \infty) ; E^{\theta}\right)\right)$ в себя. Действительно, при $t \geqslant T$ имеем

$$
\begin{aligned}
& \left\|(-A)^{\theta} G\left(v^{-} ; v\right)(t)\right\|_{E} \leqslant M_{1} e^{-t \beta}\left\|\left(I-P\left(\sigma^{+}\right)\right) v^{-}\right\|_{E^{\theta}}+ \\
& \quad+M_{1} e^{-\beta(t-T)} \int_{0}^{T} \frac{e^{-\beta(T-s)}}{|t-s|^{\alpha}}\left\|F_{u^{*}}(v(s))\right\|_{E} d s+\int_{T}^{\infty} M_{1} \frac{e^{-\beta|t-s|}}{|s-t|^{\theta}}\left\|F_{u^{*}}(v(s))\right\|_{E} d s .
\end{aligned}
$$

Для заданного $\epsilon>0$ выберем $T$ настолько большим, чтобы первый член и второй интеграл были меньше $\epsilon / 3$ для всех $t \geqslant T$; после этого возьмем $t$ так, чтобы первый интеграл был меньше $\epsilon / 3$.

Итак, найдется единственное решение уравнения $v(\cdot)=G\left(v^{-} ; v\right)$ в пространстве $C_{0}\left([0 ; \infty) ; E^{\theta}\right)$ и результат следует из единственности.

В доказательстве предложения 4.1.1 была использована следующая количественная версия теоремы Липшица об обратном отображении (см. [140,256]).

Лемма 4.1.1. Пусть $Y$ и $Z$ - банаховы пространства, $H \in C^{1}(Y, Z)$ и при некотором $y_{0} \in Y$ отображение $H^{\prime}\left(y_{0}\right)$ является гомеоморфизмом. Пусть $k, \sigma, \delta>0$ - такие три константы, что имеет место следующая оценка:

$$
\begin{gathered}
\left\|H^{\prime}(y)-H^{\prime}\left(y_{0}\right)\right\| \leqslant k<\sigma \leqslant \frac{1}{\left\|H^{\prime}\left(y_{0}\right)^{-1}\right\|} \quad \text { для любого } y \in \mathcal{U}_{Y}\left(y_{0} ; \delta\right), \\
\left\|H\left(y_{0}\right)\right\| \leqslant(\sigma-k) \delta .
\end{gathered}
$$


Тогда $H$ имеет единственный нуль $\bar{y} \in \mathcal{U}_{Y}\left(y_{0} ; \delta\right)$ и выполнены следующие неравенства:

$$
\begin{array}{ll}
\left\|H^{\prime}(y)^{-1}\right\| \leqslant \frac{1}{\sigma-k} & \text { для всех } y \in \mathcal{U}_{Y}\left(y_{0} ; \delta\right), \\
\left\|y_{1}-y_{2}\right\| \leqslant \frac{1}{\sigma-k}\left\|H\left(y_{1}\right)-H\left(y_{2}\right)\right\| & \text { для всех } y_{1}, y_{2} \in \mathcal{U}_{Y}\left(y_{0} ; \delta\right) .
\end{array}
$$

\section{2. ДИСКРЕТИЗАЦИЯ ОПЕРАТОРОВ И ПОЛУГРУПП}

Следующее утверждение является элементарным следствием определения 1.3 .2 (см. [244,256]).

Лемма 4.2.1. Пусть $B_{n}, B$-ограниченные линейные операторы. Сходимость $B_{n} \stackrel{\mathcal{P} \mathcal{P}}{\longrightarrow} B$ эквивалентна ограниченности $\left\|B_{n}\right\|$ и условию $B_{n} p_{n} x \stackrel{\mathcal{P}}{\longrightarrow} B x$ для всех $x \in E$. Если это имеет место, то для любого компактного множества $K \subset E$ имеем

$$
\sup _{x \in K}\left\|B_{n} p_{n} x-p_{n} B x\right\| \rightarrow 0 \quad \text { nрu } n \rightarrow \infty .
$$

Доказательство. Доказательство первого утверждения см. в $[244,256]$. Соотношение (4.2.1) докажем от противного. Предположим, что $\left\|B_{n} p_{n} x^{n}-p_{n} B x^{n}\right\| \geqslant \varepsilon>0$ для некоторой последовательности $x^{n} \in K, n \in \mathbb{N}$, и некоторого $\varepsilon>0$. Взяв такую подпоследовательность $x^{n}, n \in \mathbb{N}^{\prime} \subset \mathbb{N}$, что $x^{n} \rightarrow x$ для некоторого $x \in K$, приходим к противоречию с соотношением

$$
\left\|B_{n} p_{n} x^{n}-p_{n} B x^{n}\right\| \leqslant\left\|B_{n}\right\|\left\|p_{n}\left(x^{n}-x\right)\right\|+\left\|B_{n} p_{n} x-p_{n} B x\right\|+\left\|p_{n} B\left(x-x^{n}\right)\right\| \rightarrow 0 \quad \text { при } n \in \mathbb{N}^{\prime} .
$$

В качестве простого следствия получим равномерную сходимость на компактных множествах (доказательство проводиться так же, как для (4.2.1)).

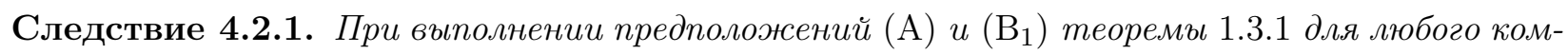
пактного множества $K \subset E$ имеем

$$
\max _{u^{0} \in K} \max _{\eta \in \Sigma(\theta, \mu)}\left\|\left(\exp \left(\eta A_{n}\right) p_{n}-p_{n} \exp (\eta A)\right) u^{0}\right\| \rightarrow 0 \quad \text { npu } n \rightarrow \infty .
$$

Полудискретная аппроксимация задачи (4.1.1) - это следующие задачи Коши в банаховых пространствах $E_{n}$ :

$$
\begin{aligned}
& u_{n}^{\prime}(t)=A_{n} u_{n}(t)+g_{n}(t), \quad t \in[0, T], \\
& u_{n}(0)=u_{n}^{0},
\end{aligned}
$$

где операторы $A_{n}$ порождают $C_{0}$-полугруппы, $A_{n}$ и $A$ согласованы, $u_{n}^{0} \stackrel{\mathcal{P}}{\longrightarrow} u^{0}$ и $g_{n}(\cdot) \stackrel{\mathcal{P}}{\longrightarrow} g(\cdot)$ в подходящем смысле. Для типичных полудискретизаций естественно предположить, что выполнены условия типа $(\mathrm{A})$ и $\left(\mathrm{B}_{1}\right)$.

4.2.1. Оценки для линейного случая. Пусть множество $\Lambda$ компактно и $\Lambda \subset \rho(A)$. По лемме 3.5.3 найдется такая константа $n_{\Lambda}>0$, что $\Lambda \subset \rho\left(A_{n}\right)$ для всех $n \geqslant n_{\Lambda}$ и

$$
\sup _{\substack{\lambda \in \Lambda, n \geqslant n_{\Lambda}}}\left\|\left(\lambda I_{n}-A_{n}\right)^{-1}\right\|<\infty .
$$

Рассмотрим контур $G$, состоящий из $\{\lambda: \operatorname{Re} \lambda=-\tilde{\omega}\}$ и части границы $\partial \Sigma\left(\pi-\phi, \omega_{3}\right)$ (см. рис. 3 , с. 48). Имеем представление

$$
\exp \left(t A_{n}\right)=\frac{1}{2 \pi i} \int_{G} e^{\lambda t}\left(\lambda I_{n}-A_{n}\right)^{-1} d \lambda .
$$

Используя оценку (3.5.12) резольвенты $\left\|\left(\lambda I_{n}-A_{n}\right)^{-1}\right\|$ и равномерную ограниченность на $G$, получим оценку (3.5.11) при $t>0$ (см. (4.1.13)).

Далее, введем операторы

$$
p_{n}^{\theta}=\left(-A_{n}\right)^{-\theta} p_{n}(-A)^{\theta} \in B\left(E^{\theta}, E_{n}^{\theta}\right)
$$


и покажем, что они обладают свойством (1.3.1), но для пространств $E^{\theta}$ и $E_{n}^{\theta}$. Будем писать $x_{n} \stackrel{\mathcal{P}^{\theta}}{\longrightarrow} x$ тогда и только тогда, когда $\left\|x_{n}-p_{n}^{\theta} x\right\|_{E_{n}^{\theta}} \rightarrow 0$ при $n \rightarrow \infty$. Очевидно, имеем

$$
\begin{gathered}
\left\|x_{n}-p_{n}^{\theta} x\right\|_{E_{n}^{\theta}}=\left\|\left(-A_{n}\right)^{\theta} x_{n}-p_{n}(-A)^{\theta} x\right\|_{E_{n}}, \\
\left\|p_{n}^{\theta} x\right\|_{E_{n}^{\theta}}=\left\|p_{n}(-A)^{\theta} x\right\|_{E_{n}} \rightarrow\left\|(-A)^{\theta} x\right\|_{E_{n}}=\|x\|_{E^{\theta}} \quad \text { при } n \rightarrow \infty
\end{gathered}
$$

для любого $x \in D\left((-A)^{\theta}\right)$, так что свойство (1.3.1) выполнено.

Для нелинейного результата нам нужна теорема о равномерной сходимости для линейных неоднородных задач с компактными данными.

Теорема 4.2.1. Пусть $A: D(A) \subset E \rightarrow E$-замкнутый оператор, удовлетворлющий условию (1.1.3) (в частности, $A$ порождает экспоненииально убывающую полугруппу). Предположим, что для аппроксимационной системы (4.2.3) выполнены условие $\Delta_{c c} \neq \varnothing$, а такэе усло-

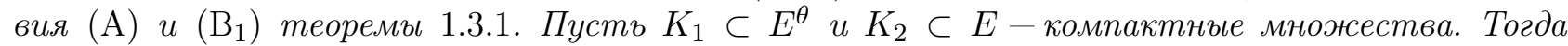
для любого $\varepsilon>0$ существуют такие $n_{1}=n_{1}(\varepsilon) \in \mathbb{N} u \delta=\delta(\varepsilon)>0$, что следующее свойство имеет место для всех $0<T \leqslant \infty$ : для любого решения $u(t)$ задачи (4.1.1) с начальными данными $u(0)=u^{0} \in K_{1}$ и $g(\cdot) \in C\left([0, T] ; K_{2}\right)$ и любого решения $u_{n}(t)$ задачи $(4.2 .3)$ с начальными данными $u_{n}(0)=p_{n}^{\theta} u^{0}$ и $g_{n}(\cdot) \in C\left([0, T] ; E_{n}\right)$ при выполнении условия

$$
\left\|g_{n}(t)-p_{n} g(t)\right\|_{E_{n}} \leqslant \delta \quad \text { при } t \in[0, T], n \geqslant n_{1},
$$

имеем оценку

$$
\left\|u_{n}(t)-p_{n}^{\theta} u(t)\right\|_{E_{n}^{\theta}} \leqslant \epsilon \quad \text { для всех } n \geqslant n_{1}, 0 \leqslant t \leqslant T .
$$

Доказательство. Обозначим через $u(t), u(0)=u^{0}$, и $u_{n}\left(t ; u_{n}^{\theta}\right), u_{n}^{\theta}=p_{n}^{\theta} u^{0}$ обобщенные решения задач (4.1.1) и (4.2.3) соответственно и пусть функции $g, g_{n}$ удовлетворяют условиям теоремы. Тогда имеют место следующие соотношения:

$$
\begin{array}{ll}
u\left(t ; u^{0}\right)=\exp (t A) u^{0}+\int_{0}^{t} \exp ((t-s) A) g(s) d s, & t \in[0, T], \\
u_{n}\left(t ; u_{n}^{\theta}\right)=\exp \left(t A_{n}\right) u_{n}^{\theta}+\int_{0}^{t} \exp \left((t-s) A_{n}\right) g_{n}(s) d s, & t \in[0, T] .
\end{array}
$$

По теореме 3.5.2 имеем

$$
\left\|\exp \left(t A_{n}\right)\right\| \leqslant M e^{-\omega t}, \quad \omega>0, \quad t \geqslant 0 .
$$

Следовательно, для любого $\epsilon>0$ найдется такое $T_{\epsilon}$, что

$$
\left\|\left(\exp \left(t A_{n}\right) p_{n}^{\theta}-p_{n}^{\theta} \exp (t A)\right) u^{0}\right\|_{E_{n}^{\theta}} \leqslant M e^{-\omega t}\left\|u^{0}\right\|_{E^{\theta}} \leqslant \epsilon, \quad t \geqslant T_{\epsilon},
$$

и согласно следствию 4.2 .1 найдется такое $n\left(T_{\epsilon}\right)$, что

$$
\left\|\left(-A_{n}\right)^{\theta}\left(\exp \left(t A_{n}\right) p_{n}^{\alpha}-p_{n}^{\alpha} \exp (t A)\right) u^{0}\right\|_{E_{n}} \leqslant \epsilon, \quad t \in\left[0, T_{\epsilon}\right], \quad n \geqslant n\left(T_{\epsilon}\right) .
$$

Заметим, что в случае $T_{\epsilon}>T$ требуется оперировать компактностью. Аналогичное замечание касается также следующей оценки интегралов. Для нашего случая продолжим функции $g(t)$ и $g_{n}(t)$ константами при $t \geqslant T$ и приведем доводы для $0 \leqslant t<\infty$. Рассмотрим $E_{n}^{\theta}$-норму разности 
интегралов, т.е.

$$
\begin{aligned}
& \left(-A_{n}\right)^{\theta} \int_{0}^{t}\left(\exp \left((t-s) A_{n}\right) g_{n}(s)-p_{n}^{\theta} \exp ((t-s) A) g(s)\right) d s= \\
& =\left(-A_{n}\right)^{\theta} \int_{0}^{t} \exp \left((t-s) A_{n}\right)\left(g_{n}(s)-p_{n} g(s)\right) d s+ \\
& +\left(-A_{n}\right)^{\theta} \int_{0}^{t}\left(\exp \left((t-s) A_{n}\right) p_{n}-p_{n}^{\theta} \exp ((t-s) A)\right) g(s) d s
\end{aligned}
$$

Первое слагаемое можно оценить следующим образом:

$$
\begin{aligned}
\left(-A_{n}\right)^{\theta}\left(\int_{0}^{t_{1}}+\int_{t_{1}}^{t}\right)=\left(-A_{n}\right)^{\theta} & \int_{0}^{t_{1}} \exp \left(s A_{n}\right)\left(g_{n}(t-s)-p_{n} g(t-s)\right) d s+ \\
& +\left(-A_{n}\right)^{\theta} \int_{0}^{t-t_{1}} \exp \left(\left(t_{1}+\eta\right) A_{n}\right)\left(g_{n}\left(t-t_{1}-\eta\right)-p_{n} g\left(t-t_{1}-\eta\right)\right) d \eta
\end{aligned}
$$

Согласно (3.5.11), выбором достаточно большого $t_{1}$ можно добиться, чтобы второе слагаемое стало меньше $\varepsilon$ равномерно по $n$. Тогда первое слагаемое с конечным $t_{1}$ можно сделать малым при помощи мажорантного члена $\left\|g_{n}(t)-p_{n} g(t)\right\|_{E_{n}} \leqslant \delta$ при $n \geqslant n_{1}$. Второе слагаемое в (4.2.8) для любого $0<t_{1} \leqslant t$ можно переписать аналогичным образом:

$$
\begin{aligned}
& \left(-A_{n}\right)^{\theta} \int_{0}^{t}\left(\exp \left(\eta A_{n}\right) p_{n}^{\theta}-p_{n}^{\theta} \exp (\eta A)\right) g(t-\eta) d \eta= \\
& =\left(-A_{n}\right)^{\alpha} \int_{0}^{t_{1}}\left(\exp \left(\eta A_{n}\right) p_{n}-p_{n}^{\theta} \exp (\eta A) B\right) g(t-\eta) d \eta+ \\
& \quad+\left(-A_{n}\right)^{\theta} \int_{0}^{t-t_{1}}\left(\exp \left(\left(t_{1}+\eta\right) A_{n}\right) p_{n}-p_{n}^{\theta} \exp \left(\left(t_{1}+\eta\right) A\right)\right) g\left(t-t_{1}-\eta\right) d \eta
\end{aligned}
$$

Снова выберем $t_{1}$ так, чтобы второе слагаемое стало малым равномерно по $n$. Тогда согласно следствию 4.2.1 первое слагаемое сходится к 0 при конечном $t_{1}$, поскольку $g(\xi)$ лежит в компактном множестве $K_{2}$. Второе слагаемое можно разложить на две части:

$$
\begin{gathered}
\left(-A_{n}\right)^{\theta} \exp \left(t_{1} A_{n}\right) \int_{0}^{t-t_{1}}\left(\exp \left(\eta A_{n}\right) p_{n}-p_{n}^{\theta} \exp (\eta A)\right) g\left(t-t_{1}+\eta\right) d \eta, \\
\left(-A_{n}\right)^{\theta}\left(\exp \left(t_{1} A_{n}\right) p_{n}^{\theta}-p_{n}^{\theta} \exp \left(t_{1} A\right)\right) \int_{0}^{t-t_{1}} \exp (\eta A) g\left(t-t_{1}+\eta\right) d \eta .
\end{gathered}
$$

Обе части можно сделать малыми, выбирая надлежащим образом $t_{1}$ и используя $(3.5 .11),(4.1 .13)$ и равномерную оценку интегралов.

4.2.2. Оценки для нелинейного случая. Рассмотрим в банаховом пространстве $E_{n}^{\theta}$ семейство задач Коши

$$
\begin{aligned}
& u_{n}^{\prime}(t)=A_{n} u_{n}(t)+f_{n}\left(u_{n}(t)\right), \quad t \geqslant 0, \\
& u_{n}(0)=u_{n}^{0} \in E_{n}^{\theta}
\end{aligned}
$$


где $u_{n}^{0} \stackrel{\mathcal{P}^{\theta}}{\longrightarrow} u^{0}$ и операторы $\left(A_{n}, A\right)$ согласованы. Предположим, что нелинейные отображения $f_{n}(\cdot): E_{n}^{\theta} \rightarrow E_{n}$ обладают следующими свойствами:

$(\mathrm{F} 2)$ отображения $f_{n}(\cdot)$ непрерывно дифференцируемы в $\mathcal{U}_{E_{n}^{\theta}}\left(p_{n}^{\theta} u^{*}, \rho\right)$, и если $x_{n} \in \mathcal{U}_{E_{n}^{\theta}}\left(p_{n}^{\theta} u^{*}, \rho\right)$ и $x_{n} \stackrel{\mathcal{P}^{\theta}}{\longrightarrow} x$, то $f_{n}\left(x_{n}\right) \stackrel{\mathcal{P}}{\longrightarrow} f(x)$ и $f_{n}^{\prime}\left(x_{n}\right) \stackrel{\mathcal{P}^{\theta} \mathcal{P}}{\longrightarrow} f^{\prime}(x)$

(F3) для любого $\epsilon>0$ найдется такое $\delta>0$, что

$$
\left\|f_{n}^{\prime}\left(w_{n}\right)-f_{n}^{\prime}\left(z_{n}\right)\right\|_{B\left(E_{n}^{\theta}, E_{n}\right)} \leqslant \epsilon \quad \text { при } \quad\left\|w_{n}-z_{n}\right\|_{E_{n}^{\theta}} \leqslant \delta
$$

для всех $w_{n}, z_{n} \in \mathcal{U}_{E_{n}^{\theta}}\left(p_{n}^{\theta} u^{*} ; \rho\right)$.

При сделанных предположениях обобщенное решение задачи (4.2.9) существует на максимальном интервале $[0, \tau)$ в $\mathcal{U}_{E_{n}^{\theta}}\left(p_{n}^{\theta} u^{*}, \rho\right)$ (см. $\left.[136,262]\right)$; обозначим его $u_{n}(\cdot)=S_{n}(\cdot) u_{n}^{0}: \overline{\mathbb{R}}^{+} \rightarrow E_{n}$. Нелинейная полугруппа $S_{n}^{n}(\cdot)$ удовлетворяет формуле вариации постоянной

$$
S_{n}(t) u_{n}^{0}=\exp \left(t A_{n}\right) u_{n}^{0}+\int_{0}^{t} \exp \left((t-s) A_{n}\right) f_{n}\left(S_{n}(s) u_{n}^{0}\right) d s, \quad t \in[0, \tau) .
$$

Рассмотрим семейство нелинейных задач (3.3.1) и введем обозначение $\mathcal{E}_{n}=\left\{u_{n}^{*} \in D\left(A_{n}\right)\right.$ : $\left.A_{n} u_{n}^{*}+f_{n}\left(u_{n}^{*}\right)=0\right\}$.

$\mathrm{C}$ этого момента будем рассматривать гиперболическую точку $u^{*}$ и соответствующие неподвижные точки $u_{n}^{*} \stackrel{\mathcal{P}^{\theta}}{\longrightarrow} u^{*}$ (см. предложение 3.3 .3$)$. Вблизи равновесия $u_{n}^{*}$ положим $u_{n}(t)=u_{n}^{*}+v_{n}(t)$; тогда задача (4.2.9) примет вид

$$
v_{n}^{\prime}(t)=A_{u_{n}^{*}, n} v_{n}(t)+F_{u_{n}^{*}, n}\left(v_{n}(t)\right), \quad v_{n}(0)=v_{n}^{0}, \quad t \geqslant 0,
$$

где

$$
A_{u_{n}^{*}, n}=A_{n}+f_{n}^{\prime}\left(u_{n}^{*}\right), \quad F_{u_{n}^{*}, n}\left(w_{n}\right)=f_{n}\left(v_{n}(t)+u_{n}^{*}\right)-f_{n}\left(u_{n}^{*}\right)-f_{n}^{\prime}\left(u_{n}^{*}\right) w_{n} .
$$

Разложим $E_{n}^{\theta}$, используя спектральные проекторы

$$
P_{n}\left(\sigma_{n}^{+}\right):=P_{n}\left(\sigma_{n}^{+}, A_{u_{n}^{*}, n}\right):=\frac{1}{2 \pi i} \int_{\partial U\left(\sigma_{n}^{+}\right)}\left(\zeta I_{n}-A_{u_{n}^{*}, n}\right)^{-1} d \zeta,
$$

где $\partial U\left(\sigma_{n}^{+}\right)$- граница области $\left\{\lambda \in \mathbb{C}: \operatorname{Re} \lambda \geqslant 0, \lambda \notin \Sigma\left(\pi-\phi, \omega_{2}\right)\right\}$, где $0<\theta<\pi / 2$, и $\omega_{2}$ задано условием $\left(\mathrm{B}_{1}\right)$ для оператора $A_{u_{n}^{*}, n}$ (ср. теорему 3.5.2). Заметим, что часть контура $\partial U\left(\sigma_{n}^{+}\right)$, лежащая на $i \mathbb{R}$, не пересекает $\sigma\left(A_{u_{n}^{*}, n}\right)$ в силу леммы 3.5.3. В частности, отсюда следует, что неподвижные точки $u_{n}^{*}$ являются гиперболическими.

Обозначим через $\sigma_{n}^{+}$часть $\sigma\left(A_{u_{n}^{*}, n}\right)$, лежащую внутри контура $\partial U\left(\sigma_{n}^{+}\right)$. Из представлений (3.1.8) и (4.2.13) получим

$$
P_{n}\left(\sigma_{n}^{+}\right) \stackrel{\mathcal{P} \mathcal{P}}{\longrightarrow} P\left(\sigma^{+}\right) \quad \text { компактно при } n \rightarrow \infty .
$$

Чтобы в этом убедиться, модифицируем контур в (3.1.8) так, чтобы он совпал с контуром в (4.2.13) и воспользуемся сходимостью

$$
\left(\zeta I_{n}-A_{u_{n}^{*}, n}\right)^{-1} \stackrel{\mathcal{P P}}{\longrightarrow}\left(\zeta I-A_{u^{*}}\right)^{-1} \quad \text { при } \zeta \in \partial U\left(\sigma^{+}\right)
$$

(см. (3.2.6)) и тем фактом, что сходимость $\left(\zeta I_{n}-A_{u_{n}^{*}, n}\right)^{-1} p_{n} x \stackrel{\mathcal{P}}{\longrightarrow}\left(\zeta I-A_{u^{*}}\right)^{-1} x$ равномерна при $\zeta \in \partial U\left(\sigma^{+}\right)$. Кроме того, из условия $\Delta_{c c} \neq \varnothing$ следует компактная сходимость проекторов и, следовательно, согласно (3.2.6) и теореме 3.2.4 получаем, что $\operatorname{dim} P_{n}\left(\sigma_{n}^{+}\right)=\operatorname{dim} P\left(\sigma^{+}\right)$при $n \geqslant n_{0}$.

Применяя теорему 3.5.2 к полугруппе $\exp \left(t A_{u_{n}^{*}, n}\right)$, получим такие константы $M_{2}, \tilde{\beta}>0$, что

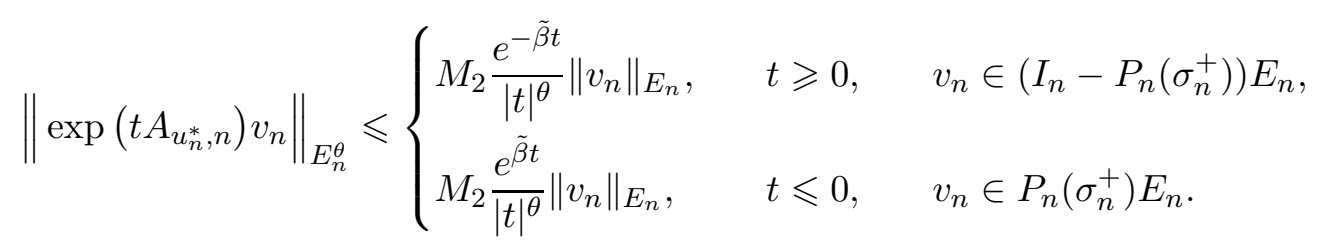


Аналогично (4.1.10) рассмотрим для любых $v_{n}^{-}, v_{n}^{+} \in \mathcal{U}_{E_{n}^{\theta}}(0 ; \rho)$ краевую задачу

$$
\begin{gathered}
v_{n}^{\prime}(t)=A_{u_{n}^{*}, n} v_{n}(t)+F_{u_{n}^{*}, n}\left(v_{n}(t)\right), \quad 0 \leqslant t \leqslant T, \\
\left(I_{n}-P_{n}\left(\sigma_{n}^{+}\right)\right) v_{n}(0)=\left(I_{n}-P_{n}\left(\sigma_{n}^{+}\right)\right) v_{n}^{-}, \quad P_{n}\left(\sigma_{n}^{+}\right) v_{n}(T)=P_{n}\left(\sigma_{n}^{+}\right) v_{n}^{+},
\end{gathered}
$$

где случай $T=\infty$ включается обычным образом. Благодаря условию (F2) можно утверждать, что обобщенное решение задачи (4.2.16) при $0 \leqslant t \leqslant T$ удовлетворяет уравнению

$$
\begin{aligned}
v_{n}(t)=\exp \left((t-T) A_{u_{n}^{*}, n}\right) P_{n}\left(\sigma_{n}^{+}\right) v_{n}^{+}+\exp \left(t A_{u_{n}^{*}, n}\right)\left(I_{n}-\right. & \left.P_{n}\left(\sigma_{n}^{+}\right)\right) v_{n}^{-}+ \\
& +\int_{0}^{T} \Gamma_{T, n}(t, s) F_{u_{n}^{*}, n}\left(v_{n}(s)\right) d s,
\end{aligned}
$$

где $\Gamma_{T, n}(t, s)$ - функция Грина:

$$
\Gamma_{T, n}(t, s)= \begin{cases}\exp \left((t-s) A_{u^{*}, n}\right)\left(I_{n}-P_{n}\left(\sigma_{n}^{+}\right)\right), & 0 \leqslant s \leqslant t \leqslant T, \\ \exp \left((t-s) A_{u^{*}, n}\right) P_{n}\left(\sigma_{n}^{+}\right), & 0 \leqslant t<s \leqslant T .\end{cases}
$$

В случае $T=\infty$ слагаемое с $v_{n}^{+}$в (4.2.17) исчезает. Следующее утверждение является аналогом предложения 4.1.1.

Предложение 4.2.1 (см. [79]). Пусть выполнены сбормулированные выше предположения относительно операторов $A, A_{n}$ и условия (F2), (F3). Тогда существует такое $\tilde{\rho}>0$, что для любого $0<\tilde{\rho}_{2} \leqslant \tilde{\rho}$ найдется $0<\tilde{\rho}_{1} \leqslant \tilde{\rho}_{2}$, обладающее следующим свойством: уравнение (4.2.17) имеет единственное решение $v_{n}(\cdot)=v_{n}\left(v_{n}^{-}, v_{n}^{+}, \cdot\right) \in C\left([0, T] ; \mathcal{U}_{E_{n}^{\theta}}\left(0 ; \tilde{\rho}_{2}\right)\right)$ для всех $v_{n}^{-}, v_{n}^{+} \in \mathcal{U}_{E_{n}^{\theta}}\left(0 ; \tilde{\rho}_{1}\right)$ и всех $0<T \leqslant \infty$. Если $T=\infty$, mo $\left\|v_{n}(t)\right\|_{E_{n}^{\theta}} \rightarrow 0$ nри $t \rightarrow \infty$.

Доказательство. Повторим доказательство предложения 4.1.1 для пространства непрерывных ограниченных функций $C\left([0, T] ; E_{n}^{\theta}\right)$ с операторами $A_{u_{n}^{*}, n}, F_{u_{n}^{*}, n}(\cdot), P_{n}\left(\sigma_{n}^{+}\right)$и $G_{n}\left(v_{n}^{-}, v_{n}^{+} ; \cdot\right)$, определенными правой частью (4.2.17). Заметим, что условие (F3) гарантирует, что можно добиться независимости констант от $n$ в оценках в (4.2.15) и, следовательно, лемма 4.1.1 выполняется равномерно по данным. Кроме того, оценки (4.1.16) и (4.1.17) также выполняются равномерно по $n$. Из (4.1.18) найдем такую константу $C^{*}>0$, что для любых двух $v_{n}, w_{n} \in C\left([0, T] ; \mathcal{U}_{E_{n}^{\theta}}\left(0 ; \tilde{\rho}_{2}\right)\right)$ имеем оценку

$$
\left\|v_{n}-w_{n}\right\| \leqslant C^{*}\left\|v_{n}-G_{n}\left(v_{n}^{-}, v_{n}^{+} ; v_{n}\right)-\left(w_{n}-G_{n}\left(v_{n}^{-}, v_{n}^{+} ; w_{n}\right)\right)\right\|
$$

здесь $\|\cdot\|=\|\cdot\|_{C\left([0, T] ; E_{n}^{\theta}\right)}$.

\section{3. ЗАТЕНЕНИЕ ПО ПРОСТРАНСТВУ}

Помимо $p_{n}, p_{n}^{\theta}$ нам потребуются дискретизирующие отображения, адаптированные к гиперболическому расщеплению. Во-первых, заметим, что спектральные проекторы $P=P\left(\sigma^{+}\right)$и $P_{n}=P_{n}\left(\sigma_{n}^{+}\right)$конечномерны и $P_{n} \stackrel{\mathcal{P P}}{\longrightarrow} P$ компактно (см. (4.2.14)). Введем дискретизирующие отображения

$$
\begin{array}{ll}
\tilde{p}_{n}: E \rightarrow E_{n}, & \tilde{p}_{n}=P_{n} p_{n} P+\left(I_{n}-P_{n}\right) p_{n}(I-P), \\
\tilde{p}_{n}^{\theta}: E^{\theta} \rightarrow E_{n}^{\theta}, & \tilde{p}_{n}^{\theta} x= \begin{cases}\left(A_{u_{n}^{*}, n}\right)^{-\theta} P_{n} p_{n}\left(A_{u^{*}}\right)^{\theta} P x, & x \in P E^{\theta}, \\
\left(-A_{u_{n}^{*}, n}\right)^{-\theta}\left(I_{n}-P_{n}\right) p_{n}\left(-A_{u^{*}}\right)^{\theta}(I-P) x, & x \in(I-P) E^{\theta} .\end{cases}
\end{array}
$$

Отметим, что спектры операторов $A_{u^{*}}=A+f^{\prime}\left(u^{*}\right)$ и $A_{u_{n}^{*}, n}=A_{n}+f_{n}^{\prime}\left(u_{n}^{*}\right)$ разделены таким образом, что дробные степени операторов определены корректно. 
Предложение 4.3.1 (см. [79]). Система $\left\{\tilde{p}_{n}\right\}$ эквивалентна системе $\left\{p_{n}\right\}$ на E и система $\left\{\tilde{p}_{n}^{\theta}\right\}$ эквивалентна системе $\left\{p_{n}^{\theta}\right\}$ на $E^{\theta}$. В частности,

$$
\begin{gathered}
\left\|\tilde{p}_{n}\right\|_{B\left(E, E_{n}\right)} \leqslant \tilde{C}, \quad\left\|\tilde{p}_{n}^{\theta}\right\|_{B\left(E^{\theta}, E_{n}^{\theta}\right)} \leqslant \tilde{C}_{\theta} \quad \text { для всех } n \in \mathbb{N}, \\
\sup _{x \in K_{2}}\left\|\left(p_{n}-\tilde{p}_{n}\right) x\right\| \rightarrow 0, \quad \sup _{x \in K_{1}}\left\|\left(p_{n}^{\theta}-\tilde{p}_{n}^{\theta}\right) x\right\| \rightarrow 0 \quad \text { nрu } n \rightarrow \infty
\end{gathered}
$$

для компактных множеств $K_{2} \subset E, K_{1} \subset E^{\theta}$.

Доказательство. Из равенства

$$
\left(\tilde{p}_{n}-p_{n}\right) x=2\left(P_{n} p_{n}-p_{n} P\right) P x-\left(P_{n} p_{n}-p_{n} P\right) x
$$

находим, что система $\left\{\tilde{p}_{n}\right\}$ эквивалентна системе $\left\{p_{n}\right\}$ на $E$. В $\theta$-случае на $(I-P) E^{\theta}$ имеет место следующая цепочка равенств:

$$
\begin{aligned}
& \begin{array}{c}
p_{n}^{\theta}-\tilde{p}_{n}^{\theta}=\left(-A_{n}\right)^{-\theta}\left(p_{n}-\tilde{p}_{n}\right)(-A)^{\theta}+\left(-A_{n}\right)^{-\theta} \tilde{p}_{n}(-A)^{\alpha}-\left(-A_{u_{n}^{*}, n}\right)^{-\theta} \tilde{p}_{n}\left(-A_{u^{*}}\right)^{\theta}= \\
=\left(-A_{n}\right)^{-\theta}\left(p_{n}-\tilde{p}_{n}\right)(-A)^{\theta}+\left(\left(-A_{n}\right)^{-\theta}-\left(-A_{u_{n}^{*}, n}\right)^{-\theta}\right) \tilde{p}_{n}(-A)^{\theta}+ \\
+\left(-A_{u_{n}^{*}, n}\right)^{-\theta} \tilde{p}_{n}\left((-A)^{\theta}-\left(-A_{u^{*}}\right)^{\theta}\right)= \\
=\left(-A_{n}\right)^{-\theta}\left(p_{n}-\tilde{p}_{n}\right)(-A)^{\theta}+\left(\left(-A_{n}\right)^{-\theta}-\left(-A_{u_{n}^{*}, n}\right)^{-\theta}\right) \tilde{p}_{n}(-A)^{\theta}+ \\
\quad+\left(-A_{u_{n}^{*}, n}\right)^{-\theta} \tilde{p}_{n}\left((-A)^{\theta}-\left(-A_{u^{*}}\right)^{\theta}\right)= \\
=\left(-A_{n}\right)^{-\theta}\left(p_{n}-\tilde{p}_{n}\right)(-A)^{\theta}+\left(-A_{u_{n}^{*}, n}\right)^{-\theta}\left(\left(-A_{u_{n}^{*}, n}\right)^{\theta}\left(-A_{n}\right)^{-\theta}-I_{n}\right) \tilde{p}_{n}(-A)^{\theta}- \\
-\left(-A_{u_{n}^{*}, n}\right)^{-\theta} \tilde{p}_{n}\left(\left(-A_{u^{*}}\right)^{\theta}(-A)^{-\theta}-I\right)(-A)^{\theta}= \\
=\left(-A_{n}\right)^{-\theta}\left(p_{n}-\tilde{p}_{n}\right)(-A)^{\theta}\left(-A_{u_{n}^{*}, n}\right)^{-\theta}+\left(\left(\left(-A_{u_{n}^{*}, n}\right)^{\theta}\left(-A_{n}\right)^{-\theta}-I_{n}\right) \tilde{p}_{n}-\right. \\
\left.\quad-\tilde{p}_{n}\left(\left(-A_{u^{*}}\right)^{\theta}(-A)^{-\theta}-I\right)\right)(-A)^{\theta} .
\end{array}
\end{aligned}
$$

На последнем шаге покажем, что

$$
\left(-A_{u_{n}^{*}, n}\right)^{\theta}\left(-A_{n}\right)^{-\theta} \stackrel{\mathcal{P P}}{\longrightarrow}\left(-A_{u^{*}}\right)^{\theta}(-A)^{-\theta} .
$$

С этой целью, следуя [136], рассмотрим формулу

$$
\begin{aligned}
\left(-A_{u^{*}}\right)^{-\theta}-(-A)^{-\theta}= & \frac{\sin (\pi \theta)}{\pi} \int_{0}^{\infty} z^{-\theta}\left(\left(z I+A_{u^{*}}\right)^{-1}-(z I+A)^{-1}\right) d z= \\
& =\frac{\sin (\pi \theta)}{\pi} \int_{0}^{\infty} z^{-\theta}\left(\left(z I+A_{u^{*}}\right)^{-1}\left(f^{\prime}\left(u^{*}\right)(-A)^{-\theta}\right)(-A)^{\theta}(z I+A)^{-1}\right) d z
\end{aligned}
$$

Поскольку

$$
\left\|(-A)^{\theta}(z I+A)^{-1}\right\|=O\left(|z|^{\theta-1}\right) \quad \text { при } z \rightarrow \infty,
$$

интеграл будет сходиться даже если подставить $\left(-A_{u^{*}}\right)^{\theta}$. Следовательно, сходимость

$$
\left(-A_{u_{n}^{*}, n}\right)^{\theta}\left(-A_{n}\right)^{-\theta} \stackrel{\mathcal{P} \mathcal{P}}{\longrightarrow}\left(-A_{u^{*}}\right)^{\theta}(-A)^{-\theta}
$$

вытекает из

$$
f_{n}^{\prime}\left(u_{n}^{*}\right)\left(-A_{n}\right)^{-\theta} \stackrel{\mathcal{P P}}{\longrightarrow} f^{\prime}\left(u^{*}\right)(-A)^{-\theta}
$$

и теоремы Лебега о мажорантной сходимости. Равномерная сходимость на компактных множествах получается, как в лемме 4.2.1. 
Замечание 4.3.1. Нетрудно показать, что $(-A)^{\theta}\left(-A_{u^{*}}\right)^{-\theta}$ - ограниченный оператор и

$$
\left(-A_{n}\right)^{\theta}\left(-A_{u_{n}^{*}, n}\right)^{-\theta} \stackrel{\mathcal{P P}}{\longrightarrow}(-A)^{\theta}\left(-A_{u^{*}}\right)^{-\theta} .
$$

Действительно,

$$
\left\|(-A)^{\theta}\left(z I-A_{u^{*}}\right)^{-1}\right\|=O\left(|z|^{\theta-1}\right) \quad \text { при } z \rightarrow \infty ;
$$

следовательно, меняя местами $A$ и $A_{u^{*}}$ в (4.3.5), получим ограниченность и сходимость так же, как в предложении 4.3.1.

Предложение 4.3.2. Пусть $0<\theta<\gamma \leqslant 1$ и выполнены условия (F1)-(F3). Тогда

$$
\sup _{w \in \mathcal{U}_{E^{\gamma}(0 ; \rho)}}\left\|F_{u_{n}^{*}, n}\left(\tilde{p}_{n}^{\theta} w\right)-\tilde{p}_{n} F_{u^{*}}(w)\right\|_{E_{n}} \rightarrow 0 \quad \text { npu } n \rightarrow \infty .
$$

Доказательство. Во-первых заметим, что

$$
\begin{aligned}
F_{u_{n}^{*}, n}\left(\tilde{p}_{n}^{\theta} w\right)-\tilde{p}_{n} F_{u^{*}}(w)=f_{n}\left(u_{n}^{*}+\tilde{p}_{n}^{\theta} w\right)-\tilde{p}_{n} f\left(u^{*}+w\right)- & \\
& -\left(f_{n}\left(u_{n}^{*}\right)-\tilde{p}_{n} f\left(u^{*}\right)+f_{n}^{\prime}\left(u_{n}^{*}\right) \tilde{p}_{n}^{\theta} w-\tilde{p}_{n} f^{\prime}\left(u^{*}\right) w\right),
\end{aligned}
$$

и, согласно предложению 4.3.1, можем заменить отображения $\tilde{p}_{n}, \tilde{p}_{n}^{\theta}$ отображениями $p_{n}, p_{n}^{\theta}$. Из предложения 3.3 .3 и условия (F2) получим

$$
f\left(u_{n}^{*}+p_{n}^{\theta} w\right) \stackrel{\mathcal{P}}{\longrightarrow} f\left(u^{*}+w\right), \quad f_{n}\left(u_{n}^{*}\right) \stackrel{\mathcal{P}}{\longrightarrow} f\left(u^{*}\right), \quad f_{n}^{\prime}\left(u_{n}^{*}\right) \stackrel{\mathcal{P}^{\theta} \mathcal{P}}{\longrightarrow} f^{\prime}\left(u^{*}\right) .
$$

Следуя схеме доказательства соотношения (4.2.1), покажем, что сходимость является равномерной на компактных множествах в $E^{\theta}$.

4.3.1. Затенение по пространству и условие $\Delta_{c c} \neq \varnothing$. Первый результат касается аппроксимации орбит общего эволюционного уравнения (4.1.5) подходящими орбитами пространственно дискретизированной системы (4.2.9).

Теорема 4.3.1 (см. [79]). Пусть А-генератор экспоненииально убывающей аналитической $C_{0}$-полугруппы $и 0 \leqslant \theta<\gamma<1$. Относительно дискретизированной системы (4.2.9) предположим, что линейные части удовлетворяют условию $\Delta_{c c} \neq \varnothing$ и условию $\left(\mathrm{B}_{1}\right)$, а нелинейные части - условиям (F1), (F2), (F3). Тогда существует число $\rho_{0}>0$, обладающее следующим свойством: для любого $\varepsilon_{0}>0$ найдется такое $n_{0}=n_{0}\left(\varepsilon_{0}\right) \in \mathbb{N}$, что для любого обобщенного решения $u(t)$ задачи (4.1.5) класса $u(t) \in \mathcal{U}_{E \gamma}\left(u^{*}, \rho_{0}\right), 0 \leqslant t \leqslant T$, для некоторого $0<T \leqslant \infty$ существуют такие начальные значения $u_{n}^{0} \in E_{n}^{\theta}, n \geqslant n_{0}$, что обобщенное решение $u_{n}\left(t ; u_{n}^{0}\right)$ задачи (4.2.9) существует на отрезке $[0, T]$ и удовлетворяет условию

$$
\sup _{0 \leqslant t \leqslant T}\left\|p_{n}^{\theta} u(t)-u_{n}\left(t ; u_{n}^{0}\right)\right\|_{E_{n}^{\theta}} \leqslant \varepsilon_{0} \quad \forall n \geqslant n_{0}(\varepsilon) .
$$

Доказательство. Условия на $n_{0}$ и $\rho_{0}$ будут сформулированы в процессе доказательства. Пусть $u(t), 0 \leqslant t \leqslant T$, 一 такое обобщенное решение задачи (4.1.5), что $u(t) \in \mathcal{U}_{E^{\gamma}}\left(u^{*}, \rho_{0}\right)$ для всех $0 \leqslant t \leqslant T$. Тогда $v(t):=u(t)-u^{*} \in \mathcal{U}_{E^{\gamma}}\left(0, \rho_{0}\right)$ - решение задачи (4.1.11), для которого

$$
v^{-}=(I-P) v(0), \quad v^{+}=P v(T) .
$$

Выбирая нормы (4.1.9), имеем

$$
\left\|v^{-}\right\|_{E^{\gamma}} \leqslant \rho_{0}, \quad\left\|v^{+}\right\|_{E^{\gamma}} \leqslant \rho_{0} .
$$

Применим предложение 4.1.1, заменив $\theta$ на $\gamma$ и положив $\hat{\rho}_{2}=\hat{\rho}$. Взяв $\rho_{0} \leqslant \hat{\rho}_{1}$, получим $\rho_{0} \leqslant \hat{\rho}_{2}$. В силу единственности в классе $C\left([0, T] ; \mathcal{U}_{E^{\gamma}}\left(0, \hat{\rho}_{2}\right)\right)$ решение $v\left(v^{-}, v^{+}, \cdot\right)$ из предложения 4.1.1 удовлетворяет равенству $v(t)=v\left(v^{-}, v^{+}, t\right), 0 \leqslant t \leqslant T$. Далее определим дискретные граничные значения

$$
v_{n}^{-}=\tilde{p}_{n}^{\theta} v^{-}, \quad v_{n}^{+}=\tilde{p}_{n}^{\theta} v^{+} .
$$


Согласно определению (4.3.2) имеем

$$
v_{n}^{-}=\tilde{p}_{n}^{\theta} v^{-}=\left(I_{n}-P_{n}\right) \tilde{p}_{n}^{\theta} v^{-}, \quad v_{n}^{+}=\tilde{p}_{n}^{\theta} v^{+}=P_{n} \tilde{p}_{n}^{\theta} v^{+},
$$

а из (4.3.3) получаем

$$
\left\|v_{n}^{ \pm}\right\|_{E_{n}^{\theta}} \leqslant \tilde{C}_{\theta}\left\|v^{ \pm}\right\|_{E^{\theta}} \leqslant \tilde{C}_{\theta} \rho_{0} .
$$

Применим предложение 4.2.1, положив $\tilde{\rho}_{2}=\tilde{\rho}$ и $\tilde{C}_{\theta} \rho_{0} \leqslant \tilde{\rho}_{1}\left(\tilde{\rho}_{2}\right)$. Взяв соответствующие единственные решения задач $(4.2 .17)$

$$
v_{n}(\cdot)=v_{n}\left(v_{n}^{-}, v_{n}^{+}, \cdot\right) \in C\left([0, T] ; \mathcal{U}_{E_{n}^{\theta}}\left(0, \tilde{\rho}_{2}\right)\right)
$$

можем утверждать, что

$$
u_{n}^{0}=u_{n}^{*}+v_{n}(0), \quad u_{n}(t)=v_{n}(t)+u_{n}^{*}
$$

удовлетворяет теореме. Потребуем, чтобы $\tilde{C}_{\theta} \rho_{0} \leqslant \tilde{\rho}_{1}$, так что можно применить $(4.2 .19)$ к $v_{n}(t)$ и $w_{n}(t)=\tilde{p}_{n}^{\theta} v(t)$. Получим

$$
\sup _{0 \leqslant t \leqslant T}\left\|v_{n}(t)-\tilde{p}_{n}^{\theta} v(t)\right\|_{E_{n}^{\theta}} \leqslant C^{*} \sup _{0 \leqslant t \leqslant T}\left\|\eta_{n}^{-}(t)+\eta_{n}^{+}(t)\right\|_{E_{n}^{\theta}}
$$

слагаемые в правой части задаются формулами

$$
\begin{aligned}
& \eta_{n}^{-}(t)=\tilde{p}_{n}^{\theta} \exp \left(t A_{u^{*}}\right)(I-P) v^{-}-\exp \left(t A_{u_{n}^{*}, n}\right)\left(I_{n}-P_{n}\right) v_{n}^{-}+ \\
& +\tilde{p}_{n}^{\theta} \int_{0}^{t} \exp \left((t-s) A_{u^{*}}\right)(I-P) F_{u^{*}}(v(s)) d s-\int_{0}^{t} \exp \left((t-s) A_{u_{n}^{*}, n}\right)\left(I_{n}-P_{n}\right) F_{u_{n}^{*}, n}\left(\tilde{p}_{n}^{\theta} v(s)\right) d s, \\
& \eta_{n}^{+}(t)=\tilde{p}_{n}^{\theta} \exp \left((t-T) A_{u^{*}}\right) P v^{+}-\exp \left((t-T) A_{u_{n}^{*}, n}\right) P_{n} v_{n}^{+}+ \\
& \quad+\tilde{p}_{n}^{\theta} \int_{t}^{T} \exp \left((t-s) A_{u^{*}}\right) P F_{u^{*}}(v(s)) d s-\int_{t}^{T} \exp \left((t-s) A_{u_{n}^{*}, n}\right) P_{n} F_{u_{n}^{*}, n}\left(\tilde{p}_{n}^{\theta} v(s)\right) d s .
\end{aligned}
$$

Оценим $\eta_{n}^{+}$, применив теорему 4.2.1, в которой положим

$$
\begin{gathered}
\tilde{E}=(I-P) E, \quad D(\tilde{A})=D(A) \cap \tilde{E}, \quad \tilde{A}=A_{u^{*}}, \\
g(t)=(I-P) F_{u^{*}}(v(t)), \quad g_{n}(t)=\left(I_{n}-P_{n}\right) F_{u_{n}^{*}, n}\left(\tilde{p}_{n}^{\theta} v(t)\right), \\
K_{1}=\mathcal{U}_{E^{\gamma}}\left(0, \rho_{0}\right), \quad K_{2}=\left\{(I-P) F_{u^{*}}(w): w \in \mathcal{U}_{E^{\gamma}}\left(0, \rho_{0}\right)\right\}, \\
\tilde{\varepsilon}=\frac{\varepsilon_{0}}{4 C^{*}}, \quad u^{0}=v^{-}, \\
\tilde{E}_{n}=\left(I_{n}-P_{n}\right) E_{n}, \quad \tilde{E}_{n}^{\theta}=\left(I_{n}-P_{n}\right) E_{n}^{\theta}, \\
u_{n}(0)=\tilde{p}_{n}^{\theta} u(0)=\tilde{p}_{n}^{\theta} v^{-} .
\end{gathered}
$$

Отметим, что в силу непрерывности функции $F_{u^{*}}: E^{\theta} \rightarrow E$ и компактного вложения $E^{\gamma}$ в $E^{\theta}$ множество $K_{1}$ компактно в $E^{\theta}$ и $K_{2}$ компактно в $E$. Оценку (4.2.5) можно применить при $n \geqslant n_{3}=\max \left(n_{1}(\tilde{\varepsilon}), n_{2}\right)$, где $n_{2}$ выбрано согласно предложению 4.3 .2 так, чтобы для $n \geqslant n_{2}$

$$
\begin{aligned}
\sup _{0 \leqslant t \leqslant T}\left\|g_{n}(t)-\tilde{p}_{n} g(t)\right\|_{\tilde{E}_{n}} \leqslant \sup _{0 \leqslant t \leqslant T} \|\left(I-P_{n}\right) & \left(F_{u_{n}^{*}, n}\left(\tilde{p}_{n}^{\theta} v(t)\right)-p_{n} F_{u^{*}}(v(t))\right) \|_{E_{n}}+ \\
& +\sup _{w \in K_{2}}\left\|\left(I-P_{n}\right)\left(p_{n} P-P_{n} p_{n}\right) F_{u^{*}}(w)\right\|_{E_{n}} \leqslant \delta=\delta(\tilde{\varepsilon}) .
\end{aligned}
$$

Следовательно, получаем

$$
\left\|\eta_{n}^{-}\right\|_{E_{n}^{\theta}} \leqslant \tilde{\varepsilon}
$$


и аналогичную оценку для $\left\|\eta_{n}^{+}\right\|_{E_{n}^{\theta}}$ и $n \geqslant n_{4}$. Окончательно, согласно (4.3.12) и предложению 3.3 .3 получим для некоторого $n_{5} \geqslant n_{4}$ и всех $0 \leqslant t \leqslant T, n \geqslant n_{5}$ оценку

$$
\left\|u_{n}(t)-p_{n}^{\theta} u(t)\right\|_{E_{n}^{\theta}} \leqslant\left\|v_{n}(t)-p_{n}^{\theta} v(t)\right\|_{E_{n}^{\theta}}+\left\|u_{n}^{*}-p_{n}^{\theta} u^{*}\right\|_{E_{n}^{\theta}} \leqslant \frac{\varepsilon_{0}}{2}+\frac{\varepsilon_{0}}{2}=\varepsilon_{0} .
$$

В следующей лемме мы аппроксимируем векторы компактной последовательности в дискретных пространствах дискретизациями непрерывных элементов.

Лемма 4.3.1. Пусть $0 \leqslant \theta<\gamma<1$ и пусть $\left\{v_{n}^{0}\right\}$-ограниченная последовательность в $E_{n}^{\gamma}$. Тогда для любого $\varepsilon>0$ найдется такое число $n_{0}(\varepsilon)$, что

$$
\inf _{v \in E^{\theta}}\left\|v_{n}^{0}-\tilde{p}_{n}^{\theta} v\right\|_{E_{n}^{\theta}} \leqslant \varepsilon, \quad n \geqslant n_{0}(\varepsilon) .
$$

Кроме того, если $0 \leqslant \theta \leqslant \beta<\gamma<1$ и последовательность $\left\{v_{n}^{0}\right\}$ лежст в $P_{n} E_{n}^{\gamma}$ и удовлетворяет условию $\left\|v_{n}^{0}\right\|_{E_{n}^{\gamma}} \leqslant b$, то существуют такие $n_{1}(\varepsilon)$ и константа $\hat{C}>0$, что

$$
\inf _{\substack{v \in P E^{\beta} \\\|v\|_{E^{\beta}} \leqslant \hat{C} b}}\left\|v_{n}^{0}-p_{n}^{\theta} v\right\|_{E_{n}^{\theta}} \leqslant \varepsilon \quad \text { для всех } n \geqslant n_{1}(\varepsilon) .
$$

Если вместо $v_{n}^{0} \in P_{n} E_{n}^{\gamma}$ выполняется условие $v_{n}^{0} \in\left(I_{n}-P_{n}\right) E_{n}^{\gamma}$, то имеет место неравенство (4.3.13), в котором инфимум берется по $v \in(I-P) E^{\beta},\|v\|_{E^{\beta}} \leqslant \hat{C} b$.

Доказательство. Из предложения 4.3.1 следует, что достаточно доказать утверждение с $p_{n}^{\theta}$ вместо $\tilde{p}_{n}^{\theta}$. Предположим, что утверждение неверно. Тогда существуют такие последовательность $\left\{v_{n}^{0}\right\}$, число $\varepsilon>0$ и подпоследовательность $\mathbb{N}^{\prime} \subseteq \mathbb{N}$, что

$$
\left\|\left(-A_{n}\right)^{\gamma} v_{n}^{0}\right\| \leqslant b, \quad\left\|v_{n}^{0}-p_{n}^{\theta} v\right\|_{E_{n}^{\theta}}>\varepsilon
$$

для всех $n \in \mathbb{N}^{\prime}, v \in E^{\theta}$. Во-первых, последовательность $(-A)_{n}^{-\theta}(-A)_{n}^{\theta} v_{n}^{0}=v_{n}^{0}$ является $\mathcal{P}$ компактной и, следовательно, найдутся такие $\mathbb{N}^{\prime \prime} \subseteq \mathbb{N}^{\prime}$ и $\bar{v} \in E$, что $v_{n}^{0} \stackrel{\mathcal{P}}{\longrightarrow} \bar{v}$ при $n \in \mathbb{N}^{\prime \prime}$. Поскольку $0 \leqslant \theta<\gamma<1$ и

$$
\left(-A_{n}\right)^{\theta-\gamma}\left(-A_{n}\right)^{\gamma} v_{n}^{0}=\left(-A_{n}\right)^{\theta} v_{n}^{0}
$$

получим аналогичным образом, что $\left(-A_{n}\right)^{\theta} v_{n}^{0}$ является $\mathcal{P}$-компактной. Таким образом, найдется такое подмножество $\mathbb{N}^{\prime \prime \prime} \subseteq \mathbb{N}^{\prime \prime}$, что $\left(-A_{n}\right)^{\theta} v_{n}^{0} \stackrel{\mathcal{P}}{\longrightarrow} z \in E$ при $n \in \mathbb{N}^{\prime \prime \prime}$, и мы заключаем, что

$$
\left(-A_{n}\right)^{-\theta}\left(-A_{n}\right)^{\theta} v_{n}^{0} \stackrel{\mathcal{P}}{\longrightarrow}(-A)^{-\theta} z \quad \text { при } n \in \mathbb{N}^{\prime \prime \prime} .
$$

С другой стороны, $v_{n}^{0} \stackrel{\mathcal{P}}{\longrightarrow} \bar{v}$ при $n \in \mathbb{N}^{\prime \prime}$, откуда следует, что $\bar{v}=(-A)^{-\theta} z \in E^{\theta}$. Окончательно имеем

$$
\left\|v_{n}^{0}-p_{n}^{\theta} \bar{v}\right\|_{E_{n}^{\theta}}=\left\|\left(-A_{n}\right)^{\theta} v_{n}^{0}-p_{n} z\right\|_{E_{n}} \rightarrow 0 \quad \text { при } n \in \mathbb{N}^{\prime \prime \prime} \text { для некоторого } \bar{v} \in E^{\theta},
$$

противоречие. Для доказательства второго утверждения разовьем предыдущие идеи следующим образом, Во-первых, согласно лемме 4.2.1, для каждого $0 \leqslant \lambda<1$ найдется такая константа $C_{[\lambda]}>0$, что

$$
\left\|(-A)^{-\lambda}\right\|_{B(E, E)}, \quad\left\|\left(-A_{n}\right)^{-\lambda}\right\|_{B\left(E_{n}, E_{n}\right)} \leqslant C_{[\lambda]} \quad \text { для всех } n \in \mathbb{N} .
$$

Пусть $\hat{C}=C_{[\gamma-\beta]}+1$. Выше было установлено, что $\left(-A_{n}\right)^{\beta} v_{n}^{0} \stackrel{\mathcal{P}}{\longrightarrow} y \in E$ при $n \in \mathbb{N}^{\prime \prime \prime}$ и тогда $\bar{v}=(-A)^{-\beta} y \in E^{\beta}$. Используя включение $v_{n}^{0} \in P_{n} E_{n}^{\gamma}$ и сходимость проекторов (4.2.14), находим

$$
0=\left(I_{n}-P_{n}\right) v_{n}^{0} \stackrel{\mathcal{P}}{\longrightarrow} \bar{v}-P \bar{v} ;
$$

таким образом, $\bar{v} \in P E^{\beta}$. Кроме того, для больших $n \in \mathbb{N}^{\prime \prime \prime}$ имеем

$$
\|\bar{v}\|_{E^{\beta}}=\|y\|_{E} \leqslant\left\|\left(-A_{n}\right)^{\beta-\gamma}\left(-A_{n}\right)^{\gamma} v_{n}^{0}\right\|_{E_{n}}+b \leqslant\left(C_{[\gamma-\beta]}+1\right) b=\hat{C} b .
$$

Это противоречит неравенству $\left\|v_{n}^{0}-p_{n}^{\theta} v\right\|>\varepsilon, v \in P E^{\beta},\|v\|_{E^{\beta}} \leqslant \hat{C} b$.

Эта лемма будет использована ниже для построения подходящих граничных условий для следующего обратного результата о затенении. 
Теорема 4.3.2 (см. [79]). Пусть выполняются предположения теоремь 4.3.1. Тогда существует $\rho_{0}>0$, обладающее следующим свойством: для любого $\varepsilon_{0}>0$ найдется такое $n_{0}=$ $n_{0}\left(\varepsilon_{0}\right) \in \mathbb{N}$, что для любого обобщенного решения $u_{n}(t), n \geqslant n_{0}$, задачи (4.2.9), удовлетворяющего условию $u_{n}(t) \in \mathcal{U}_{E_{n}^{\gamma}}\left(u_{n}^{*}, \rho_{0}\right), 0 \leqslant t \leqslant T$, для некоторого $0<T \leqslant \infty$, существуют такие начальные значения $u^{n, 0} \in E^{\theta}, n \geqslant n_{0}$, что обобщенное решение $u\left(t ; u^{n, 0}\right)$ задачи (4.1.5) существует на отрезке $[0, T]$ и удовлетворяет оценке

$$
\sup _{0 \leqslant t \leqslant T}\left\|u_{n}(t)-p_{n}^{\theta} u\left(t ; u^{n, 0}\right)\right\|_{E_{n}^{\theta}} \leqslant \varepsilon_{0} \quad \forall n \geqslant n_{0}\left(\varepsilon_{0}\right) .
$$

Доказательство. Как и в теореме 4.3.1, возьмем некоторое $\varepsilon_{0}>0$; условия на $n_{0}$ и $\rho_{0}$ будут сформулированы в процессе доказательства. Рассмотрим обобщенное решение $u_{n}(t), 0 \leqslant t \leqslant T$, задачи (4.2.9), лежащее в $\mathcal{U}_{E_{n}^{\gamma}}\left(u_{n}^{*}, \rho_{0}\right)$, и определим

$$
v_{n}(t)=u_{n}(t)-u_{n}^{*}, \quad v_{n}^{-}=\left(I_{n}-P_{n}\right) v_{n}(0), \quad v_{n}^{+}=P_{n} v_{n}(T) .
$$

Благодаря равномерной ограниченности проекторов имеем для некоторого $C_{b} \geqslant 1$

$$
\left\|v_{n}^{-}\right\|_{E_{n}^{\gamma}} \leqslant C_{b} \rho_{0}, \quad\left\|v_{n}^{+}\right\|_{E_{n}^{\gamma}} \leqslant C_{b} \rho_{0} .
$$

Применим предложение 4.2 .1 к элементам $v_{n}^{ \pm}$, взяв $\gamma$ вместо $\theta$ и $\tilde{\rho}_{2}=\tilde{\rho}$. Потребуем, чтобы $C_{b} \rho_{0} \leqslant \tilde{\rho}_{1}$, так что соотношение $v_{n}(t)=v_{n}\left(v_{n}^{-}, v_{n}^{+}, t\right), 0 \leqslant t \leqslant T$, имеет место в силу единственности решений в $C\left([0, T] ; \mathcal{U}_{E_{n}^{\gamma}}(0 ; \tilde{\rho})\right)$. Теперь возьмем $\theta<\beta<\gamma$ и, применяя лемму 4.3.1, построим такие граничные значения $v^{n,-} \in(I-P) E^{\beta}, v^{n,+} \in P E^{\beta}, n \geqslant n_{1}\left(\varepsilon_{0}\right)$, чтобы

$$
\left\|v_{n}^{-}-\tilde{p}_{n}^{\theta} v^{n,-}\right\|_{E_{n}^{\theta}}+\left\|v_{n}^{+}-\tilde{p}_{n}^{\theta} v^{n,+}\right\|_{E_{n}^{\theta}} \leqslant \frac{\varepsilon_{0}}{16 M_{2} C^{*}}, \quad\left\|v^{n, \pm}\right\|_{E^{\beta}} \leqslant \hat{C} \rho_{0}
$$

(см. (4.2.15), (4.2.19)). На следующем шаге применим предложение 4.1.1 с граничными значениями $v^{n, \pm}$ и $\beta$ вместо $\theta$. Выберем $\hat{\rho}_{2}$ так, чтобы (cр. (4.3.14), (4.3.3))

$$
\tilde{C}_{\theta} C_{[\beta-\theta]} \hat{\rho}_{2} \leqslant \tilde{\rho}_{2},
$$

потребуем, чтобы $\left(C_{[\gamma-\theta]}+\hat{C}\right) \rho_{0} \leqslant \tilde{\rho}_{2}$, и обозначим единственное решение в $C\left([0, T] ; \mathcal{U}_{E^{\beta}}\left(0 ; \hat{\rho}_{2}\right)\right)$ через $v^{n}(t), 0 \leqslant t \leqslant T$. Покажем, что

$$
u^{n, 0}=v^{n}(0)+u^{*}, \quad u\left(t ; u^{n, 0}\right)=v^{n}(t)+u^{*}, \quad 0 \leqslant t \leqslant T,
$$

удовлетворяет (4.3.7). Для этого подставим $v_{n}(\cdot)$ и $w_{n}(\cdot):=\tilde{p}_{n}^{\theta} v^{n}(\cdot)$ в (4.2.19). Это неравенство справедливо, поскольку

$$
\begin{gathered}
\left\|v_{n}(t)\right\|_{E_{n}^{\theta}} \leqslant C_{[\gamma-\theta]} \rho_{0}, \\
\left\|w_{n}(t)\right\|_{E_{n}^{\theta}} \leqslant \tilde{C}_{\theta}\left\|v^{n}(t)\right\|_{E^{\theta}} \leqslant \tilde{C}_{\theta} C_{[\beta-\theta]}\left\|v^{n}(t)\right\|_{E^{\beta}} \leqslant \tilde{C}_{\theta} C_{[\beta-\theta]} \hat{\rho}_{2} \leqslant \tilde{\rho}_{2}, \\
\left\|v_{n}^{ \pm}\right\|_{E_{n}^{\theta}} \leqslant C_{[\gamma-\theta]}\left\|v_{n}^{ \pm}\right\|_{E_{n}^{\gamma}} \leqslant C_{[\gamma-\theta]} C_{b} \rho_{0} \leqslant \tilde{\rho}_{1} .
\end{gathered}
$$

Получим оценку

$$
\begin{aligned}
\left\|v_{n}-w_{n}\right\|_{C\left([0, T] ; E_{n}^{\theta}\right)} \leqslant C^{*}\left\|w_{n}-G_{n}\left(v_{n}^{-}, v_{n}^{+}, w_{n}\right)\right\|_{C\left([0, T] ; E_{n}^{\theta}\right)} \leqslant & \\
& \leqslant C^{*} \sup _{0 \leqslant t \leqslant T}\left\|\eta_{n}^{-}(t)+\eta_{n}^{+}(t)+\varphi_{n}^{-}(t)+\varphi_{n}^{+}(t)\right\|_{E_{n}^{\theta}},
\end{aligned}
$$

где слагаемые в правой части задаются формулами

$$
\begin{aligned}
& \eta_{n}^{-}(t)=\tilde{p}_{n}^{\theta} \exp \left(t A_{u^{*}}\right)(I-P) v^{n,-}-\exp \left(t A_{u_{n}^{*}, n}\right)\left(I_{n}-P_{n}\right) \tilde{p}_{n}^{\theta} v^{n,-}+ \\
& \quad+\tilde{p}_{n}^{\theta} \int_{0}^{t} \exp \left((t-s) A_{u^{*}}\right)(I-P) F_{u^{*}}\left(v^{n}(s)\right) d s-\int_{0}^{t} \exp \left((t-s) A_{u_{n}^{*}, n}\right)\left(I_{n}-P_{n}\right) F_{u_{n}^{*}, n}\left(\tilde{p}_{n}^{\theta} v^{n}(s)\right) d s,
\end{aligned}
$$




$$
\begin{aligned}
& \eta_{n}^{+}(t)=\tilde{p}_{n}^{\theta} \exp \left((t-T) A_{u^{*}}\right) P v^{n,+}-\exp \left((t-T) A_{u_{n}^{*}, n}\right) P_{n} \tilde{p}_{n}^{\theta} v^{n,+}+ \\
& \quad+\tilde{p}_{n}^{\theta} \int_{t}^{T} \exp \left((t-s) A_{u^{*}}\right) P F_{u^{*}}\left(v^{n}(s)\right) d s-\int_{t}^{T} \exp \left((t-s) A_{u_{n}^{*}, n}\right) P_{n} F_{u_{n}^{*}, n}\left(\tilde{p}_{n}^{\theta} v^{n}(s)\right) d s \\
& \varphi_{n}^{-}(t)=\exp \left(t A_{u_{n}^{*}, n}\right)\left(I_{n}-P_{n}\right)\left(\tilde{p}_{n}^{\theta} v^{n,-}-v_{n}^{-}\right), \quad \varphi_{n}^{+}(t)=\exp \left((t-T) A_{u_{n}^{*}, n}\right) P_{n}\left(\tilde{p}_{n}^{\theta} v^{n,+}-v_{n}^{+}\right) .
\end{aligned}
$$

Почти так же, как в теореме 4.3.1, при помощи теоремы 4.2.1 получим оценку

$$
\left\|\eta_{n}^{ \pm}\right\| \leqslant \frac{\varepsilon_{0}}{8 C^{*}}
$$

(cp. (4.3.12)); главное различие состоит в том, что $v(s)$ заменено на $v^{n}(s)$ и компактные множества задаются теперь как

$$
K_{2}=\left\{(I-P) F_{u^{*}}(w): w \in \mathcal{U}_{E^{\beta}}\left(0 ; \hat{\rho}_{2}\right)\right\}, \quad K_{1}=\mathcal{U}_{E^{\beta}}\left(0 ; \hat{C} \rho_{0}\right)
$$

(см. (4.3.19)).

Для второго слагаемого имеем по теореме 3.5.2 и (4.3.19)

$$
\left\|\varphi_{n}^{-}(t)+\varphi_{n}^{+}(t)\right\|_{E_{n}^{\theta}} \leqslant M_{2} e^{-\tilde{\beta} t} \frac{\varepsilon_{0}}{8 M_{2} C^{*}} \leqslant \frac{\varepsilon_{0}}{8 C^{*}} .
$$

Отсюда, из (4.3.21) и предложения 3.3.3 окончательно имеем для больших $n$

$$
\left\|u_{n}(t)-p_{n}^{\theta} u\left(t, u^{n, 0}\right)\right\|_{E_{n}^{\theta}} \leqslant\left\|v_{n}(t)-p_{n}^{\theta} v^{n}(t)\right\|_{E_{n}^{\theta}}+\left\|u_{n}^{*}-p_{n}^{\theta} u^{*}\right\|_{E_{n}^{\theta}} \leqslant \varepsilon_{0} .
$$

\section{4. ЗАТЕНЕНИЕ ПО ВРЕМЕНИ}

В силу расщепления пространств нам потребуются кроме $p_{n}, p_{n}^{\theta}$ и другие согласующие отображения. Введем операторы

$$
\hat{p}_{n}=P_{n}\left(\sigma_{n}^{+}\right) \tilde{p}_{n}^{\theta} P\left(\sigma^{+}\right), \quad \check{p}_{n}=\left(I_{n}-P_{n}\left(\sigma_{n}^{+}\right)\right) \tilde{p}_{n}^{\theta}\left(I-P\left(\sigma^{+}\right)\right) .
$$

Поскольку операторы $P_{n}\left(\sigma_{n}^{+}\right), p_{n}$ равномерно ограничены, операторы $\check{p}_{n}, \hat{p}_{n}$ также равномерно ограничены на $E^{\theta}$.

Переформулируем теоремы 4.3 .1 и 4.3.2 следующим образом.

Теорема 4.4.1 (см. [79]). Пусть оператор А является генератором экспоненииально убывающей аналитической $C_{0}$-полугруппы и u$^{*}$ - гиперболическая точка равновесия системы (4.1.5). Пусть $\Delta_{c c} \neq \varnothing, f_{n}\left(x_{n}\right) \stackrel{\mathcal{P}}{\longrightarrow} f(x), f_{n}^{\prime}\left(x_{n}\right) \stackrel{\mathcal{P}^{\theta} \mathcal{P}}{\longrightarrow} f^{\prime}(x)$ при $x_{n} \stackrel{\mathcal{P}^{\theta}}{\longrightarrow} x$ и выполнены условия (F1)(F3). Тогда для любого обобщенного решения задачи (4.1.10) nри $v^{0}, v^{T} \in \mathcal{U}_{E^{\gamma}}(0 ; \rho)$ найдутся такие обобщеннве решения задачи (4.2.16), удовлетворяющие условиям $v_{n}^{0}=\check{p}_{n} v^{0}, v_{n}^{T}=\hat{p}_{n} v^{T}$, чmo

$$
\sup _{v^{0}, v^{T} \in \mathcal{U}_{E^{\gamma}}(0 ; \rho)} \sup _{0 \leqslant t \leqslant T}\left\|v_{n}\left(t ; v_{n}^{0}, v_{n}^{T}\right)-\bar{p}_{n}^{\theta} v\left(t ; v^{0}, v^{T}\right)\right\|_{E_{n}^{\theta}} \rightarrow 0 \quad \text { npu } n \rightarrow \infty .
$$

Теорема 4.4.2 (см. [79]). Пусть оператор А является генератором экспоненциально убъвающей аналитической $C_{0}$-полугруппы и и* - гиперболическая точка равновесия системы (4.1.5). Пусть $\Delta_{c c} \neq \varnothing, f_{n}\left(x_{n}\right) \stackrel{\mathcal{P}}{\longrightarrow} f(x), f_{n}^{\prime}\left(x_{n}\right) \stackrel{\mathcal{P}^{\theta} \mathcal{P}}{\longrightarrow} f^{\prime}(x)$ при $x_{n} \stackrel{\mathcal{P}^{\theta}}{\longrightarrow} x$ и выполнены условия (F1) и (F3). Тогда для любой последовательности обобщенных решений задач (4.2.16) при $v_{n}^{0}, v_{n}^{T} \in \mathcal{U}_{E_{n}^{\gamma}}(0 ; \rho), 0 \leqslant \theta<\gamma<1$, найдутся такие множества элементов $\left\{v^{0, E}\right\}, v^{0, E} \in E^{\theta}$, $\left\{v^{T, E}\right\}, v^{T, E} \in E^{\theta}$, что $v^{0, E}, v^{T, E} \in \mathcal{U}_{E^{\theta}}(0 ; \rho), 0 \leqslant \theta<\gamma<1$, и обобщенные решения задач (4.1.10) npu $v^{0, E}, v^{T, E} \in \mathcal{U}_{E^{\theta}}(0 ; \rho)$, umo

$$
\sup _{v_{n}^{0}, v_{n}^{T} \in \mathcal{U}_{E_{n}^{\gamma}}(0 ; \rho)} \sup _{0 \leqslant t \leqslant T}\left\|v_{n}\left(t ; v_{n}^{0}, v_{n}^{T}\right)-\bar{p}_{n}^{\theta} v\left(t ; v^{0, E}, v^{T, E}\right)\right\|_{E_{n}^{\theta}} \rightarrow 0 \quad \text { npu } n \rightarrow \infty .
$$


4.4.1. Затенение по времени и условие $\Delta_{c c} \neq \varnothing$. Рассмотрим для задачи (4.2.11) следующую дискретизационную схему по времени:

$$
\frac{V_{n}\left(t+\tau_{n}\right)-V_{n}(t)}{\tau_{n}}=A_{u_{n}^{*}, n} V_{n}\left(t+\tau_{n}\right)+F_{u_{n}^{*}, n}\left(V_{n}(t)\right), \quad t=k \tau_{n},
$$

с начальными данными $V_{n}(0)=v_{n}^{0}$. Решение такой задачи дается формулой

$$
\begin{aligned}
V_{n}(t+ & \left.\tau_{n}\right)=\left(I_{n}-\tau_{n} A_{u_{n}^{*}, n}\right)^{-1} V_{n}(t)+\tau_{n}\left(I_{n}-\tau_{n} A_{u_{n}^{*}, n}\right)^{-1} F_{u_{n}^{*}, n}\left(V_{n}(t)\right)= \\
& =\left(I_{n}-\tau_{n} A_{u_{n}^{*}, n}\right)^{-k} V_{n}(0)+\tau_{n} \sum_{j=0}^{k}\left(I_{n}-\tau_{n} A_{u_{n}^{*}, n}\right)^{-(k-j+1)} F_{u_{n}^{*}, n}\left(V_{n}\left(j \tau_{n}\right)\right), \quad t=k \tau_{n},
\end{aligned}
$$

где $V_{n}(0)=v_{n}^{0}$.

Теорема 4.4.3 (см. [214]). Пусть $\Delta_{c c} \neq \varnothing$ и резольвенты операторов $A_{n}, A$ компактны. Предположим также, что $C_{0}$-полугруппа $e^{t A_{u^{*}}}, t \in \overline{\mathbb{R}}^{+}$, является гиперболической и выполнено условие $\left(\mathrm{B}_{1}\right)$. Тогда

$$
\begin{cases}\left\|\left(I_{n}-\tau_{n} A_{u_{n}^{*}, n}\right)^{-k_{n}}\left(I_{n}-P_{n}\right)\right\|_{E_{n}} \leqslant M_{2} r^{[t]}, & t=k_{n} \tau_{n} \geqslant 0, \\ \left\|\left(I_{n}-\tau_{n} A_{u_{n}^{*}, n}\right)^{k_{n}} P_{n}\right\|_{E_{n}} \leqslant M_{2} r^{-[t]}, & t=-k_{n} \tau_{n} \leqslant 0\end{cases}
$$

где $r<1$.

Доказательство. Из компактной сходимости резольвент $\left(\lambda I_{n}-A_{n}\right)^{-1} \stackrel{\mathcal{P P}}{\longrightarrow}(\lambda I-A)^{-1}$ следует (см. [89]), что

$$
\left(\lambda I_{n}-A_{u_{n}^{*}, n}\right)^{-1} \stackrel{\mathcal{P P}}{\longrightarrow}\left(\lambda I-A_{u^{*}}\right)^{-1} \quad \text { компактно. }
$$

Положим $B_{n}=\left(I_{n}-\tau_{n} A_{u_{n}^{*}, n}\right)^{-k_{n}}, \tau_{n} k_{n}=1$, и $B=e^{1 A_{u^{*}}}$. Тогда $B_{n} \stackrel{\mathcal{P P}}{\longrightarrow} B$, поскольку операторы $A_{u_{n}^{*}, n}, A_{u^{*}}$ согласованы. Заметим, что по условию $\left(\mathrm{B}_{1}\right)$ имеем (см. $\left.[29,127]\right)$

$$
\left\|\tau_{n} k_{n} A_{u_{n}^{*}, n}\left(I_{n}-\tau_{n} A_{u_{n}^{*}, n}\right)^{-k_{n}}\right\|_{B\left(E_{n}\right)} \leqslant \text { const }
$$

откуда следует, что

$$
B_{n}=A_{u_{n}^{*}, n}^{-1} \tau_{n} k_{n} A_{u_{n}^{*}, n}\left(I_{n}-\tau_{n} A_{u_{n}^{*}, n}\right)^{-k_{n}} \stackrel{\mathcal{P} \mathcal{P}}{\longrightarrow} B \quad \text { компактно, }
$$

поскольку $A_{u_{n}^{*}, n}^{-1} \stackrel{\mathcal{P P}}{\longrightarrow} A_{u^{*}}^{-1}$ компактно. Теперь согласно теореме 4.6 .5 получаем для $B_{n}$ дискретную дихотомию. Теорема доказана.

Замечание 4.4.1. Можно рассмотреть задачу (4.4.3) со слагаемым $F_{u_{n}^{*}, n}\left(V_{n}\left(t+\tau_{n}\right)\right)$, однако такая схема не имеет большого теоретического значения.

Задачу (4.2.16) можно дискретизировать также, используя подход (4.4.3)-(4.4.4), в результате чего получим

$$
\begin{gathered}
\frac{V_{n}\left(t+\tau_{n}\right)-V_{n}(t)}{\tau_{n}}=A_{u_{n}^{*}, n} V_{n}\left(t+\tau_{n}\right)+F_{u_{n}^{*}, n}\left(V_{n}(t)\right), \quad t=k \tau_{n}, \\
\left(I_{n}-P_{n}\right) V_{n}(0)=\left(I_{n}-P_{n}\right) v_{n}^{0}, \quad P_{n} V_{n}(T)=P_{n} v_{n}^{T} .
\end{gathered}
$$

Решение задачи (4.4.6) можно получить, используя формулы

$$
\begin{gathered}
\left(I_{n}-P_{n}\right) V_{n}\left(t+\tau_{n}\right)=\left(I_{n}-\tau_{n} A_{u_{n}^{*}, n}\right)^{-1}\left(I_{n}-P_{n}\right) V_{n}(t)+\tau_{n}\left(I_{n}-\tau_{n} A_{u_{n}^{*}, n}\right)^{-1}\left(I_{n}-P_{n}\right) F_{u_{n}^{*}, n}\left(V_{n}\left(j \tau_{n}\right)\right), \\
\left(I_{n}-\tau_{n} A_{u_{n}^{*}, n}\right) P_{n} V_{n}\left(t+\tau_{n}\right)=P_{n} V_{n}(t)+\tau_{n} P_{n} F_{u_{n}^{*}, n}\left(V_{n}\left(k \tau_{n}\right)\right), \quad t=k \tau_{n} .
\end{gathered}
$$


Последнее уравнение разрешимо, поскольку $F_{u_{n}^{*}, n}\left(V_{n}\left(k \tau_{n}\right)\right)$ достаточно мало в рассматриваемой окрестности (см. условие (F3)). Таким образом, имеем представление решения задачи (4.4.6) в виде

$$
\begin{aligned}
& V_{n}\left(t ; v_{n}^{0}, v_{n}^{T}\right)=\left(I_{n}-P_{n}\right) V_{n}(t)+P_{n} V_{n}(t)= \\
& =\left(I_{n}-\tau_{n} A_{u_{n}^{*}, n}\right)^{-k+1}\left(I_{n}-P_{n}\right) v_{n}^{0}+\tau_{n} \sum_{j=0}^{k-1}\left(I_{n}-\tau_{n} A_{u_{n}^{*}, n}\right)^{-(k-j)}\left(I_{n}-P_{n}\right) F_{u_{n}^{*}, n}\left(V_{n}\left(j \tau_{n}\right)\right)+ \\
& \quad+\left(I_{n}-\tau_{n} A_{u_{n}^{*}, n}\right)^{K-k} P_{n} v_{n}^{T}-\tau_{n} \sum_{j=k}^{K-1}\left(I_{n}-\tau_{n} A_{u_{n}^{*}, n}\right)^{j-k} P_{n} F_{u_{n}^{*}, n}\left(V_{n}\left(j \tau_{n}\right)\right), \quad t=k \tau_{n} .
\end{aligned}
$$

Лемма 4.4.1. Пусть оператор А является генератором экспоненциально убывающей аналитической $C_{0}$-полугруппь и u$^{*}$-гиперболическая точка равновесия задачи (4.1.5). Пусть $\Delta_{c c} \neq \varnothing, f_{n}\left(x_{n}\right) \stackrel{\mathcal{P}}{\longrightarrow} f(x), f_{n}^{\prime}\left(x_{n}\right) \stackrel{\mathcal{P}^{\theta} \mathcal{P}}{\longrightarrow} f^{\prime}(x)$ при $x_{n} \stackrel{\mathcal{P}^{\theta}}{\longrightarrow} x$ и выполнены условия (F1)-(F3) u $\left(\mathrm{B}_{1}\right)$. Тогда найдется такая константа $C>0$, не зависящая от $n$, что

$$
\begin{gathered}
\left\|\left(I_{n}-\tau_{n} A_{u_{n}^{*}, n}\right)^{-k}\left(I_{n}-P_{n}\right)\right\| \leqslant C, \\
\tau_{n} \sum_{j=0}^{k-1}\left\|\left(-A_{n}\right)^{\theta}\left(I_{n}-\tau_{n} A_{u_{n}^{*}, n}\right)^{-(k-j)}\left(I_{n}-P_{n}\right)\right\| \leqslant C
\end{gathered}
$$

nрu $k, n \in \mathbb{N}$.

Доказательство. Согласно предложению Pconteqh с $\theta=0$ операторы $A_{u_{n}^{*}, n}$ генерируют на подпространствах $\left(I_{n}-P_{n}\right) E_{n}$ ограниченные аналитические $C_{0}$-полугруппы с равномерными оценками

$$
\left\|e^{t A_{u_{n}^{*}, n}\left(I_{n}-P_{n}\right)}\right\| \leqslant M e^{-\omega t}, \quad t \geqslant 0 .
$$

Отсюда следует

$$
\left\|\left(I_{n}-\tau_{n} A_{u_{n}^{*}, n}\right)^{-k}\left(I_{n}-P_{n}\right)\right\| \leqslant C
$$

для любых $k, n \in \mathbb{N}$ и $0<\tau_{n} \leqslant \tau^{*}$. Теперь по теореме 4.4.3 получаем дихотомические оценки (4.4.5) и используем формулу с абсолютно сходящимся интегралом

$$
\begin{aligned}
& \tau_{n} \sum_{j=0}^{k-1}\left(-A_{n}\right)^{\theta}\left(I_{n}-\tau_{n} A_{u_{n}^{*}, n}\right)^{-(k-j)}\left(I_{n}-P_{n}\right)= \\
& \quad=\left(-A_{n}\right)^{\theta}\left(-A_{u_{n}^{*}, n}\right)^{-\theta} \tau_{n} \sum_{j=0}^{k-1} \frac{1}{2 \pi i} \int_{\Gamma_{1}} \zeta^{\theta}\left(1-\tau_{n} \zeta\right)^{-(k-j)}\left(\zeta I_{n}-A_{u_{n}^{*}, n}\right)^{-1} d \zeta\left(I_{n}-P_{n}\right),
\end{aligned}
$$

где контур $\Gamma_{1}$ соответствует спектру оператора $\sigma\left(A_{u_{n}^{*}, n}\left(I_{n}-P_{n}\right)\right)$. Ясно также, что для $\zeta \in \Gamma_{1}$ имеем

$$
\left|1-\tau_{n} \zeta\right|=\sqrt{\left(1-\tau_{n} \operatorname{Re} \zeta\right)^{2}+\left(\tau_{n} \operatorname{Im} \zeta\right)^{2}} \geqslant 1-\tau_{n} \operatorname{Re} \zeta
$$

Следовательно,

$$
\begin{gathered}
\left|1-\tau_{n} \zeta\right|^{-1} \leqslant \frac{1}{1-\tau_{n} \operatorname{Re} \zeta} \\
\sum_{j=0}^{k-1}\left|1-\tau_{n} \zeta\right|^{-(k-j)} \leqslant \frac{1}{\left|1-\tau_{n} \zeta\right|} \frac{1-\tau_{n} \operatorname{Re} \zeta}{-\tau_{n} \operatorname{Re} \zeta}, \quad k, n \in \mathbb{N},
\end{gathered}
$$


при $\zeta \in \Gamma_{1}$. Интеграл в (4.4.8) абсолютно сходится. Действительно,

$$
\begin{aligned}
& \int_{0}^{q} \tau_{n} \sum_{j=0}^{k-1}|\zeta|^{\theta}\left|1-\tau_{n} \zeta\right|^{-(k-j)} \frac{M}{|\zeta|+1} d|\zeta|+\int_{q}^{\infty} \tau_{n} \sum_{j=0}^{k-1} \zeta^{\theta}\left(1-\tau_{n} \zeta\right)^{-(k-j)} \frac{M}{|\zeta|+1} d|\zeta| \leqslant \\
& \leqslant \int_{0}^{q}|\zeta|^{\theta} \frac{M}{|\zeta|+1} d|\zeta| \frac{\tau_{n}}{1-\frac{1}{1+c \tau_{n}}}+\int_{q}^{\infty}|\zeta|^{\theta} \frac{1}{1-\frac{1}{1-\tau_{n} \operatorname{Re} \zeta}} \frac{M}{|\zeta|+1} \frac{d|\zeta|}{\left|1-\tau_{n} \zeta\right|} \leqslant \\
& \leqslant \frac{\left(1+c \tau_{n}\right)}{c} \int_{0}^{q}|\zeta|^{\theta} \frac{M}{|\zeta|+1} d \zeta+\int_{q}^{\infty}|\zeta|^{\theta} \frac{\left(1-\tau_{n} \operatorname{Re} \zeta\right) d|\zeta|}{-\operatorname{Re} \zeta\left|1-\tau_{n} \zeta\right|(|\zeta|+1)}
\end{aligned}
$$

Из [79] имеем

$$
\left\|\left(A_{u_{n}^{*}, n}\right)^{\theta}\left(A_{n}\right)^{-\theta}\right\|, \quad\left\|\left(A_{n}\right)^{\theta}\left(A_{u_{n}^{*}, n}\right)^{-\theta}\right\| \leqslant C, \quad n \in \mathbb{N} .
$$

Второе утверждение доказано.

Лемма 4.4.2. Пусть оператор А является генератором экспоненииально убывающей аналитической $C_{0}$-полугруппы и и* -гиперболическая точка равновесия задачи (4.1.5). Пусть $\Delta_{c c} \neq \varnothing, f_{n}\left(x_{n}\right) \stackrel{\mathcal{P}}{\longrightarrow} f(x), f_{n}^{\prime}\left(x_{n}\right) \stackrel{\mathcal{P}^{\theta} \mathcal{P}}{\longrightarrow} f^{\prime}(x)$ при $x_{n} \stackrel{\mathcal{P}^{\theta}}{\longrightarrow} x$ и выполнены условия (F1)-(F3) u $\left(\mathrm{B}_{1}\right)$. Тогда найдется такая константа $C>0$, не зависящая от $n$, что

$$
\left\|\left(I_{n}-\tau_{n} A_{u_{n}^{*}, n}\right)^{k} P_{n}\right\| \leqslant C, \quad \tau_{n} \sum_{j=k}^{K-1}\left\|\left(-A_{n}\right)^{\theta}\left(I_{n}-\tau_{n} A_{u_{n}^{*}, n}\right)^{j-k} P_{n}\right\| \leqslant C
$$

при $K, k, n \in \mathbb{N}, k \leqslant K-1$.

Доказательство. Из (4.2.14) следует существование таких проекторов $P_{n}$, что $P_{n} \stackrel{\mathcal{P} \mathcal{P}}{\longrightarrow} P$ компактно. Используя формулы

$$
\begin{gathered}
\left(-A_{n}\right)^{\theta}\left(I_{n}-\tau_{n} A_{u_{n}^{*}, n}\right)^{j} P_{n}=\left(-A_{n}\right)^{\theta}\left(A_{u_{n}^{*}, n}\right)^{-\theta} \frac{1}{2 \pi i} \int_{\Gamma_{2}} \zeta^{\theta}\left(1-\tau_{n} \zeta\right)^{j}\left(\zeta I_{n}-A_{u_{n}^{*}, n}\right)^{-1} d \zeta P_{n}, \\
\left(-A_{n}\right)^{\theta} \tau_{n} \sum_{j=0}^{K-k-1}\left(I_{n}-\tau_{n} A_{u_{n}^{*}, n}\right)^{j} P_{n}= \\
=\left(-A_{n}\right)^{\theta}\left(A_{u_{n}^{*}, n}\right)^{-\theta} \frac{1}{2 \pi i} \int_{\Gamma_{2}} \tau_{n} \sum_{j=0}^{K-k-1} \zeta^{\theta}\left(1-\tau_{n} \zeta\right)^{j}\left(\zeta I_{n}-A_{u_{n}^{*}, n}\right)^{-1} d \zeta P_{n},
\end{gathered}
$$

получим оценки, где $\Gamma_{2}$ - конечный контур, соответствующий спектру оператора $\sigma\left(A_{u_{n}^{*}, n} P_{n}\right)$. Действительно, для любого $\zeta \in \Gamma_{2}$ имеем

$$
\left|1-\tau_{n} \zeta\right| \leqslant 1-2 \tau_{n} \operatorname{Re} \zeta+\left(\tau_{n} \operatorname{Re} \zeta\right)^{2}+\left(\tau_{n} \operatorname{Im} \zeta\right)^{2} \leqslant 1-c \tau_{n}<1, \quad \zeta \in \Gamma_{2},
$$

где $c>0$-некоторая константа и $\tau_{n}$ достаточно мало, поскольку $Г$ окружает конечную часть спектра $\sigma\left(A_{u_{n}^{*}, n} P_{n}\right)$ в правой полуплоскости. Таким образом,

$$
\tau_{n} \sum_{j=0}^{K-k-1}\left|1-\tau_{n} \zeta\right|^{j} \leqslant \tau_{n} \frac{1}{1-\left(1-c \tau_{n}\right)} \leqslant \frac{1}{c}, \quad K, k, n \in \mathbb{N}, \quad k \leqslant K-1,
$$

откуда вытекает утверждение леммы. 
Лемма 4.4.3. Пусть операторы $A_{n}$ являются генераторами равномерно по $n$ экспоненциально убъвающих аналитических $C_{0}$-полугрупn, m.e.

$$
\left\|e^{t A_{n}}\right\| \leqslant M e^{-\omega t}, \quad t \geqslant 0
$$

Тогда

$$
\left(I_{n}-\tau_{n} A_{n}\right)^{-k} \rightarrow 0, \quad\left(-A_{n}\right)^{\theta}\left(I_{n}-\tau_{n} A_{n}\right)^{-k} \rightarrow 0, \quad 0<\theta<1,
$$

nрu $0<\tau_{n} \leqslant \tau^{*}, k \rightarrow \infty, k \tau_{n} \rightarrow \infty, u$

$$
\left\|\left(\left(I_{n}-\tau_{n} A_{n}\right)^{-k}-e^{t A_{n}}\right)\left(-A_{n}\right)^{-\theta} u_{n}^{0}\right\| \leqslant C \frac{\tau_{n}^{\theta}}{k}\left\|u_{n}^{0}\right\| .
$$

Доказательство. При $t=k \tau_{n}$ рассмотрим разность

$$
\begin{gathered}
\left(I_{n}-\tau_{n} A_{n}\right)^{-k}-e^{t A_{n}}=\int_{0}^{\tau_{n}} \frac{d}{d s}\left(\exp \left(k\left(\tau_{n}-s\right) A\right)\left(I_{n}-s A_{n}\right)^{-k}\right) d s= \\
=\int_{0}^{\tau_{n}} \exp \left(k\left(\tau_{n}-s\right) A\right) k\left(-A_{n}+\frac{A_{n}}{I_{n}-s A_{n}}\right)\left(I_{n}-s A_{n}\right)^{-k} d s= \\
=\frac{1}{k} \int_{0}^{\tau_{n}} \frac{\left(\left(\tau_{n}-s\right) k\left(-A_{n}\right)\right)^{1 / 2} \exp \left(k\left(\tau_{n}-s\right) A\right)}{\sqrt{\tau_{n}-s}} \frac{\left(s k\left(-A_{n}\right)\right)^{3 / 2}\left(I_{n}-s A_{n}\right)^{-k-1}}{\sqrt{s}} d s .
\end{gathered}
$$

Известно (см. [26]), что

Видим, что

$$
\left\|\left(t\left(-A_{n}\right)\right)^{1 / 2} e^{t A_{n}}\right\| \leqslant M e^{-\omega t}, \quad t \geqslant 0 .
$$

$$
\left\|\left(k s\left(-A_{n}\right)\right)^{3 / 2}\left(I_{n}-s A_{n}\right)^{-k}\right\| \leqslant C
$$

для любых $k, n \in \mathbb{N}, k>\theta$, и $0<s \leqslant \tau^{*}$. Действительно,

$$
\begin{gathered}
\left\|\left(-A_{n}\right)^{\theta}\left(\lambda I_{n}-A_{n}\right)^{-k}\right\| \leqslant \frac{1}{(k-1) !} \int_{0}^{\infty} t^{k-1} e^{-\lambda t}\left\|\left(-A_{n}\right)^{\theta} e^{t A_{n}}\right\| d t \leqslant \frac{M \lambda^{\theta-k}}{(k-1) !} \int_{0}^{\infty} s^{k-1-\theta} e^{-s} d s, \\
\left\|\left(-A_{n}\right)^{\theta}\left(I_{n}-\tau_{n} A_{n}\right)^{-k}\right\|=\tau_{n}^{-k}\left\|\left(-A_{n}\right)^{\theta}\left(\frac{1}{\tau_{n}} I_{n}-A_{n}\right)^{-k}\right\| \leqslant M \tau_{n}^{-\theta} \frac{\Gamma(k-\theta)}{\Gamma(k)},
\end{gathered}
$$

и поскольку

$$
\lim _{k \rightarrow \infty} \frac{k^{\theta} \Gamma(k)}{\Gamma(k+\theta)}=1,
$$

получаем требуемую оценку. Теперь из (4.4.10) следует, что

$$
\left\|\left(I_{n}-\tau_{n} A_{n}\right)^{-k}\right\| \leqslant \frac{1}{k}, \quad t>0
$$

Таким образом, для гладких элементов типа $\left(-A_{n}\right)^{-\theta} u_{n}^{0}$ получаем (4.4.9).

Теорема 4.4.4 (см. [214]). Пусть оператор А является генератором экспоненииально убъвающей аналитической $C_{0}$-полугруппы и и* - гиперболическая точка равновесия задачи (4.1.5). Пусть $\Delta_{c c} \neq \varnothing, f_{n}\left(x_{n}\right) \stackrel{\mathcal{P}}{\longrightarrow} f(x), f_{n}^{\prime}\left(x_{n}\right) \stackrel{\mathcal{P}^{\theta} \mathcal{P}}{\longrightarrow} f^{\prime}(x)$ при $x_{n} \stackrel{\mathcal{P}^{\theta}}{\longrightarrow} x$ и выполнень условия $\left(\mathrm{B}_{1}\right)$, (F1)-(F3). Тогда существует $\rho_{0}>0$, обладающее следующим свойством: для любого $\epsilon_{0}$ и любого обобщенного решения $v(\cdot)$ задачи (4.1.10), для которого $v^{0}, v^{T} \in \mathcal{U}_{E^{\gamma}}\left(0 ; \rho_{0}\right), 0 \leqslant \theta<\gamma<1$, найдутся такие $n\left(\epsilon_{0}\right)$ и решения $V_{n}(\cdot)$ задач (4.4.6), для которых $v_{n}^{0}, v_{n}^{T} \in \mathcal{U}_{E_{n}^{\theta}}\left(0 ; \rho_{0}\right)$, что

$$
\sup _{0 \leqslant t \leqslant T}\left\|V_{n}\left(t ; v_{n}^{0}, v_{n}^{T}\right)-\tilde{p}_{n}^{\theta} v\left(t ; v^{0}, v^{T}\right)\right\|_{E_{n}^{\theta}} \leqslant \epsilon_{0} \quad \text { npu } n \geqslant n\left(\epsilon_{0}\right) .
$$


Доказательство. Как уже было сказано, решение задачи (4.4.6) существует; это доказывается так же, как [79, предложение 2.2]. Действительно, при $K \tau_{n}=T$ введем (аналогично [127, Sec. 5.1]) банахово пространство $X_{n}=C_{\tau_{n}}\left([0, T] ; E_{n}^{\theta}\right)$ с нормой

$$
\left\|V_{n}(\cdot)\right\|_{C_{\tau_{n}}\left([0, T] ; E_{n}^{\theta}\right)}=\max _{0 \leqslant j \leqslant K}\left\|V_{n}\left(j \tau_{n}\right)\right\|_{E_{n}^{\theta}} .
$$

Обозначим через $G\left(v_{n}^{0}, v_{n}^{T} ; V_{n}(\cdot)\right)$ оператор в правой части (4.4.7), примененный к элементу $V_{n}(\cdot) \in C_{\tau_{n}}\left([0, T] ; E_{n}^{\theta}\right)$. Для такого оператора имеем $G^{\prime}\left(v_{n}^{0}, v_{n}^{T} ; 0\right)=0$. Следовательно, оператор $I_{n}-G^{\prime}\left(v_{n}^{0}, v_{n}^{T} ; 0\right)$ обратим. Кроме того (см. аналогичные выкладки в $\left.[79,(2.17)]\right)$, для производных Фреше

$$
\left\|G^{\prime}\left(v_{n}^{0}, v_{n}^{T} ; 0\right)-G^{\prime}\left(v_{n}^{0}, v_{n}^{T} ; V_{n}(\cdot)\right)\right\|_{B\left(X_{n}\right)} \leqslant q<1,
$$

если $V_{n}(\cdot) \in \mathcal{U}_{C_{\tau_{n}}\left([0, T] ; E_{n}^{\theta}\right)}\left(0 ; \rho_{0}\right)$, поскольку ряды сходятся согласно леммам 4.4.1 и 4.4.2. Таким образом, существование единственного решения $V_{n}(\cdot)$ задачи (4.4.7) следует из [259, лемма 1].

Теперь покажем, что если $v_{n}(\cdot)$ - решение задачи $(4.2 .16)$, то

$$
\sup _{0 \leqslant t \leqslant T}\left\|v_{n}\left(t ; v_{n}^{0}, v_{n}^{T}\right)-V_{n}(t)\right\|_{E_{n}^{\theta}} \leqslant \frac{\epsilon_{0}}{3}
$$

при $n \geqslant n\left(\epsilon_{0}\right)$; результат получается из (4.9.1) с $\epsilon_{0} / 3$ в правой части. Напомним, что в силу единственности решения

Поскольку

$$
v_{n}(t)=u_{n}(t)-u_{n}^{*}, \quad v(t)=u(t)-u^{*} .
$$

$$
\begin{gathered}
v_{n}(t)-\tilde{p}_{n}^{\theta} v(t)=u_{n}(t)-\tilde{p}_{n}^{\theta} u(t)-u_{n}^{*}+\tilde{p}_{n}^{\theta} u^{*}, \\
\left\|u_{n}^{*}-\tilde{p}_{n}^{\theta} u^{*}\right\| \leqslant \frac{\epsilon_{0}}{3} \quad \text { при } n \geqslant n\left(\epsilon_{0}\right),
\end{gathered}
$$

неравенство (4.9.1) можно применить для оценивания разности $v_{n}(t)-\tilde{p}_{n}^{\theta} v(t)$. Чтобы оценить $\left\|v_{n}(t)-V_{n}\left(k \tau_{n}\right)\right\|$, рассмотрим разность правых частей в (4.4.7) и (4.2.17). Имеем (см. [24]), например, для $v_{n}^{0}$ и $\gamma-\theta>0$

$$
\begin{aligned}
& \left\|\left(-A_{n}\right)^{\theta}\left(\exp \left(t A_{u_{n}^{*}, n}\right)-\left(I_{n}-\tau_{n} A_{u_{n}^{*}, n}\right)^{-k}\right)\left(I_{n}-P_{n}\right) v_{n}^{0}\right\| \leqslant \\
& \leqslant C\left\|\left(-A_{u_{n}^{*}, n}\right)^{\theta}\left(\exp \left(t A_{u_{n}^{*}, n}\right)-\left(I_{n}-\tau_{n} A_{u_{n}^{*}, n}\right)^{-k}\right)\left(I_{n}-P_{n}\right) v_{n}^{0}\right\| \leqslant C \frac{\tau_{n}^{\gamma-\theta}}{k}\left\|\left(-A_{u_{n}^{*}, n}\right)^{\gamma} v_{n}^{0}\right\|,
\end{aligned}
$$

где первое неравенство следует из [79, замечание 4.2], а второе - это в точности (4.4.9). Оценки интегральных членов вытекают из условия (F3) (см. замечание 4.8.1) и лемм 4.4.1-4.4.2. Теперь выберем $\tau_{n}$ так, чтобы имело место (4.4.11).

Теорема 4.4.5. Пусть оператор А является генератором экспоненциально убывающей аналитической $C_{0}$-полугруппь и $u^{*}$ - гиперболическая точка равновесия задачи (4.1.5). Пусть $\Delta_{c c} \neq \varnothing, f_{n}\left(x_{n}\right) \stackrel{\mathcal{P}}{\longrightarrow} f(x), f_{n}^{\prime}\left(x_{n}\right) \stackrel{\mathcal{P}^{\theta} \mathcal{P}}{\longrightarrow} f^{\prime}(x)$ при $x_{n} \stackrel{\mathcal{P}^{\theta}}{\longrightarrow} x$ и выполненъ условия $\left(\mathrm{B}_{n}\right),(\mathrm{F} 1)-$ (F3). Тогда существует $\rho_{0}>0$, обладающее следующим свойством: для любого $\epsilon_{0}>0$ найдется такое $n_{0}=n\left(\epsilon_{0}\right) \in \mathbb{N}$, что для любого решения $V_{n}(t), n \geqslant n_{0}$, задачи (4.4.6), удовлетворяющего условию $V_{n}(t) \in \mathcal{U}_{E_{n}^{\gamma}}\left(0 ; \rho_{0}\right), 0 \leqslant t \leqslant T, 0 \leqslant \theta<\gamma<1$, u для некоторого $0<T \leqslant \infty$, для которого $v_{n}^{0}, v_{n}^{T} \in \mathcal{U}_{E_{n}^{\theta}}(0 ; \rho)$, существуют такие элементы $v^{n, 0}, v^{n, T} \in E^{\theta}, n \geqslant n_{0}$, что обобщенное решение $v\left(t ; v^{n, 0}, v^{n, T}\right)$ задачи (4.1.10) существует на отрезке $[0, T]$ и удовлетворяет оченке

$$
\sup _{0 \leqslant t \leqslant T}\left\|V_{n}\left(t ; v_{n}^{0}, v_{n}^{T}\right)-\tilde{p}_{n}^{\theta} v\left(t ; v^{n, 0}, v^{n, T}\right)\right\|_{E_{n}^{\theta}} \leqslant \epsilon_{0} \quad \forall n \geqslant n\left(\epsilon_{0}\right) .
$$

Доказательство основано на теореме 4.4.2 и оценке типа (4.4.12).

Замечание 4.4.2. Можно доказать теоремы, аналогичные теоремам 4.4.4 и 4.4.5, для схемы

$$
\frac{V_{n}\left(t+\tau_{n}\right)-V_{n}(t)}{\tau_{n}}=A_{u_{n}^{*}, n} V_{n}(t)+F_{u_{n}^{*}, n}\left(V_{n}(t)\right), \quad t=k \tau_{n}
$$


вместо схемы $(4.4 .6)$, но с некоторым условием устойчивости типа $\left\|\tau_{n} A_{u_{n}^{*}}\right\| \leqslant$ const.

\section{5. ПровеРКА УСЛОвИЯ $\Delta_{c c} \neq \varnothing$}

В этом разделе будет показано, как можно удовлетворить предположения теорем 4.3.1 и 4.3.2 для методов конечных элементов и конечных разностей.

Пример 4.5.1. Пусть $\Omega \subset \mathbb{R}^{d}$ - ограниченная гладкая область. Рассмотрим сильно эллиптический оператор второго порядка

$$
L u(x)=\sum_{i, j=1}^{d} a_{i j}(x) u_{x_{i} x_{j}}(x)+\sum_{j=1}^{d} b_{j}(x) u_{x_{j}}(x)+c(x) u(x),
$$

где коэффициенты $a_{i j}, b_{j}, c$ - гладкие ограниченные функции. Рассмотрим ассоциированную параболическую задачу

$$
\begin{array}{ll}
u_{t}(t, x)=L u(t, x)+f(u(t, x)), & t>0, \quad x \in \Omega, \\
u(t, x)=0, & t>0, \quad x \in \partial \Omega, \\
u(0, x)=u^{0}(x) \in H_{0}^{1}(\Omega) . &
\end{array}
$$

Пусть $E=L^{2}(\Omega)$; определим оператор $A: D(A) \subset E \rightarrow E$ следующим образом: $D(A)=H^{2}(\Omega) \cap$ $H_{0}^{1}(\Omega)$ и $A u=L u$ для всех $u \in D(A)$. Известно, что $A$ порождает аналитическую компактную $C_{0}$-полугруппу $\{\exp (t A): t \geqslant 0\}$. Предположим, что функция $c(x)$ выбрана так, что спектр оператора $A$ расположен левее мнимой оси. Тогда можно определить дробные степени $(-A)^{\theta}$ оператора $-A$, как было показано выше. Хорошо известно, что $E^{1}=D(A)=H^{2}(\Omega) \cap H_{0}^{1}(\Omega)$ и $E^{1 / 2}=H_{0}^{1}(\Omega)$.

Относительно нелинейного члена $f(\cdot)$ известно (см. $[52,165])$, что при выполнении некоторых условий роста задача (4.5.2) локально корректна в $E^{1 / 2}$ и оператор-функция $f(\cdot)$ дифференцируема по Фреше как функция из $E^{\theta}$ в $E$. Например, предположения могут быть следующими (см. [165]): скалярная функция $f(x, \cdot): \mathbb{R} \rightarrow \mathbb{R}, x \in \Omega$, принадлежит классу $C^{2}(\mathbb{R}, \mathbb{R})$ и для любых $\xi \in \mathbb{R}, x \in \Omega$ выполняется неравенство

$$
\left|f_{\xi}^{(l)}(x, \xi)\right| \leqslant C\left(1+|\xi|^{\delta+1-l}\right),
$$

где $l=1,2$ и $\delta=2$, если $d=3$, и $\delta \in[1, \infty)$, если $d=2$. Тогда можно показать (см. [165]), что

$$
\begin{gathered}
\left\|f^{\prime}(u)-f^{\prime}(v)\right\|_{B\left(E^{1 / 2}, E\right)} \leqslant C(\rho)\|u-v\|_{E^{1 / 2}}, \\
\left\|f(u)-f(v)-f^{\prime}(w)(u-v)\right\|_{E^{(k-1) / 2}} \leqslant C(\rho)\left(\|u-w\|_{E^{1 / 2}}+\|v-w\|_{E^{1 / 2}}\right)\|u-v\|_{E^{k / 2}}
\end{gathered}
$$

при $k=1,2$ и любых $v, w, u \in\left\{z \in E:\left\|z-u^{*}\right\|_{E^{1 / 2}} \leqslant \rho\right\}$. Из неравенств (4.5.3), (4.5.4) вытекает условие $(\mathrm{F} 1)$.

Задача (4.1.3) с указанными выше оператором $A$ и функцией $f(\cdot)$ корректно поставлена и обладает всеми свойствами, требуемыми в основной теореме.

Кроме того, аппроксимационная задача (4.2.9) также имеет нужные свойства, если она определена методом конечных элементов.

Действительно, пусть $A, E$ и $E^{1 / 2}$ такие же, как описано выше. Известно (см. [151]), что оператор $A$ находится во взаимно однозначном соответствии с полуторалинейной формой $a$ : $E^{1 / 2} \times E^{1 / 2} \rightarrow \mathbb{C}$, так что

$$
\begin{array}{ll}
|a(u, v)| \leqslant c_{1}\|u\|_{E^{1 / 2}}\|v\|_{E^{1 / 2}}, & u, v \in E^{1 / 2}, \\
\operatorname{Re} a(u, u) \geqslant c_{2}\|u\|_{E^{1 / 2}}, & u \in E^{1 / 2}, \\
a(u, v)=\langle-A u, v\rangle, & u \in D(A), v \in E^{1 / 2} .
\end{array}
$$

Рассмотрим выпуклый многоугольник $\Omega \subset \mathbb{R}^{2}$ и регулярную триангуляцию, в которой треугольники имеют максимальный диаметр $h$. Обозначим через $S_{h}$ пространство функций в $E^{1 / 2}$, линейных на каждом элементе и обращающихся в нуль на границе. Тогда $S_{h}$ является семейством 
конечномерных подпространств пространства $H_{0}^{1}(\Omega)$ со стандартным аппроксимационным свойством (см. [248])

$$
\inf _{\chi \in S_{h}}\left(\|v-\chi\|_{E}+h\|v-\chi\|_{E^{1 / 2}}\right) \leqslant C h^{2}\|v\|_{E^{1}}, \quad v \in H^{2}(\Omega) \cap H_{0}^{1}(\Omega) .
$$

Обозначим через $P_{h} u$ проекцию элемента $u \in E$ на $S_{h}=E_{h}^{1 / 2}$ относительно скалярного произведения в $L^{2}(\Omega)$. Эти операторы играют роль связывающих отображений $\left\{p_{h}\right\}$. В этом контексте конечноэлементная аппроксимации $A_{h}: S_{h} \rightarrow S_{h}$ of $A$ определенf формулой

$$
\left\langle-A_{h} \phi_{h}, \psi_{h}\right\rangle=a\left(\phi_{h}, \psi_{h}\right), \quad \phi_{h}, \psi_{h} \in E_{h}^{1 / 2} \text {. }
$$

Другими словами, $A_{h}$ - оператор, ассоциированный с полуторалинейной формой $a_{h}(\cdot, \cdot)$, которая является сужением формы $a(\cdot, \cdot)$ на $E_{h}^{1 / 2} \times E_{h}^{1 / 2}$. В этом случае можно доказать (см. [111]), что существуют такие константа $C$ и острый угол $\theta$, что при $u \in E$ и $\theta \leqslant|\arg z| \leqslant \pi$ имеем

$$
\left\|(z I-A)^{-1} u-\left(z I_{h}-A_{h}\right)^{-1} P_{h} u\right\|_{E} \leqslant C h^{2}\|u\|_{E} .
$$

Эта оценка означает $\mathcal{P}$-сходимость с равномерной сходимостью резольвент. Поскольку резольвента $(\lambda I-A)^{-1}$ компактна для некоторого $\lambda$, из приведенного выше неравенства получаем (здесь $\mu(\cdot)$ - мера некомпактности)

$$
\mu\left(\left(z I_{h}-A_{h}\right)^{-1} x_{h}\right) \leqslant \mu\left((z I-A)^{-1} x_{h}\right)+\varlimsup_{h \rightarrow 0}\left\|(z I-A)^{-1} x_{h}-\left(z I_{h}-A_{h}\right)^{-1} x_{h}\right\|_{E}=0
$$

откуда следует компактная сходимость резольвент при $h \rightarrow 0$. Таким образом, наше основное предположение $\Delta_{c c} \neq \varnothing$ может быть проверено.

Окончательно, положив $f_{h}\left(v_{h}\right)=p_{h} f\left(v_{h}\right)$ для $v_{h} \in E_{h}^{1 / 2}$ и $F_{u_{h}^{*}, h}\left(v_{h}\right)=f_{h}\left(v_{h}-u_{h}^{*}\right)-f_{h}\left(u_{h}^{*}\right)-$ $f_{h}^{\prime}\left(u_{h}^{*}\right) v_{h}$, видим, что задачи (3.3.1), (4.2.11) корректны. Из оценки (4.5.4) следует, что

$$
\left\|F_{u^{*}}(v(t))\right\|_{E} \leqslant c(\rho)\|v(t)\|_{E^{1 / 2}}^{2}
$$

кроме того, имеем $F_{u^{*}}^{\prime}(0)=0$ и условие $(\mathrm{F} 3)$ в виде

$$
\left\|F_{u_{h}^{*}, h}\left(v_{h}\right)\right\|_{E_{n}} \leqslant \tilde{c}(\rho)\left\|v_{h}\right\|_{E_{h}^{1 / 2}}^{2}
$$

поскольку норма $\left\|p_{h}\right\|$ равномерно ограничена.

Пример 4.5.2. Резольвентные оценки при условии $\left(B_{1}\right)$ были доказаны для методов конечных элементов и конечных разностей, например, в [60,68]. Не входя в детали, покажем, как получить компактную сходимость резольвент для метода конечных разностей. Рассмотрим, например, в пространстве $E=L^{2}(0,1)$ оператор $A$, определенный следующим образом:

$$
A v(x)=\frac{d^{2} v(x)}{d x^{2}}, \quad D(A)=\left\{v(\cdot) \in H_{0}^{1}(0,1) \cap H^{2}(0,1): v(0)=v(1)=0\right\}
$$

Выберем величину шага $h=1 / n$ и аппроксимируем оператор $A$ операторами

где

$$
A_{n} u_{n}=\bar{\partial}_{h} \partial_{h} u_{n}=\left\{\frac{1}{h^{2}}\left(u_{n,(k+1) h}-2 u_{n, k h}+u_{n,(k-1) h}\right)\right\}_{k=1}^{n-1}
$$

$u_{n, \cdot} \in E_{n}=L_{h}^{2}(0,1)=D\left(A_{n}\right)=\left\{\left\{u_{n, k h}\right\}_{k=1}^{n-1} \in \mathbb{R}^{n-1}\right\}$.

Заметим, что в (4.5.5) мы положили $u_{n, 0}=u_{n, n h}=0$. Связывающие отображения $p_{n}$ заданы формулами

$$
\left(p_{n} u\right)_{k h}=\frac{1}{h} \int_{k-h / 2}^{k+h / 2)} u(x) d x, \quad u \in E
$$

(см. [256]). Если скалярное произведение задано формулой

$$
\left\langle u_{n, \cdot}, v_{n, \cdot}\right\rangle_{E_{n}}=h \sum_{k=1}^{n-1} u_{n, k h} v_{n, k h}
$$


то формула суммирования по частям дает

$$
-\left\langle\bar{\partial}_{h} \partial_{h} u_{n}, u_{n}\right\rangle_{E_{n}}=\left\langle\partial_{h} u_{n}, \partial_{h} u_{n}\right\rangle_{E_{n}}=\left\|\partial_{h} u_{n}\right\|_{E_{n}}^{2}=h \sum_{k=1}^{n}\left(\frac{u_{n, k h}-u_{n,(k-1) h}}{h}\right)^{2}
$$

(мы снова учли, что $\left.u_{n, 0}=u_{n, n h}=0\right)$. Отсюда следует

$$
\left\|\partial_{h} u_{n}\right\|_{E_{n}}^{2} \leqslant\left\|A_{n} u_{n}\right\|_{E_{n}}\left\|u_{n}\right\|_{E_{n}}
$$

Таким образом, для любой ограниченной последовательности $\left\{u_{n}\right\}$, для которой $\left\{A_{n} u_{n}\right\}$ также ограничена в $E_{n}$, из последнего неравенства следует, что $\left\|u_{n}\right\|_{E_{n}^{1 / 2}}$ ограничена. Отсюда вытекает, что $\left\{u_{n}\right\}$ является $\mathcal{P}$-компактной. Теперь, применяя теорему 2.3 .1 , получим компактную сходимость резольвент.

\section{6. Дихотомия для полугрупп}

В этом разделе будет описан случай, когда резольвента оператора $A$ не обязательно является компактным оператором. Таким образом, становится возможным рассмотреть оператор $A$, имеющий не только точечный спектр.

Если $v^{0}$ близко к 0 , например, $v^{0} \in \mathcal{U}_{E^{\theta}}(0 ; \rho)$, где $\rho>0$ мало, то обобщенное решение $v\left(t ; v^{0}\right)$ задачи (4.1.6) может оставаться в пределах шара $\mathcal{U}_{E^{\theta}}(0 ; \rho)$ в течение некоторого времени. Обозначим максимальное время, в течение которого $v\left(t ; v^{0}\right)$ остается внутри $\mathcal{U}_{E^{\theta}}(0 ; \rho)$, через

$$
T=T\left(v^{0}\right)=\sup \left\{t \geqslant 0:\left\|v\left(t ; v^{0}\right)\right\|_{E^{\theta}} \leqslant \rho \text { или } v\left(t ; v^{0}\right) \in \mathcal{U}_{E^{\theta}}(0 ; \rho)\right\} .
$$

Возвращаясь к решению задачи (4.1.6), для любых двух $v^{0}, v^{T} \in \mathcal{U}_{E^{\theta}}(0 ; \rho)$ рассмотрим краевую задачу

$$
\left\{\begin{array}{l}
v^{\prime}(t)=A_{u^{*}} v(t)+F_{u^{*}}(v(t)), \quad 0 \leqslant t \leqslant T, \\
\left(I-P\left(\sigma^{+}\right)\right) v(0)=\left(I-P\left(\sigma^{+}\right)\right) v^{0}, \quad P\left(\sigma^{+}\right) v(T)=P\left(\sigma^{+}\right) v^{T} .
\end{array}\right.
$$

Как известно (см. [79]), обобщенное решение задачи (4.6.1) удовлетворяет интегральному уравнению

$$
\begin{aligned}
v(t)= & e^{(t-T) A_{u^{*}}} P\left(\sigma^{+}\right) v^{T}+e^{t A_{u^{*}}}\left(I-P\left(\sigma^{+}\right)\right) v^{0}+ \\
& +\int_{0}^{t} e^{(t-s) A_{u^{*}}}\left(I-P\left(\sigma^{+}\right)\right) F_{u^{*}}(v(s)) d s+\int_{t}^{T} e^{(t-s) A_{u^{*}}} P\left(\sigma^{+}\right) F_{u^{*}}(v(s)) d s, \quad 0 \leqslant t \leqslant T .
\end{aligned}
$$

При дискретизации задачи (4.1.6) по пространственной и временной переменным важно знать, что произойдет с оценкой типа (3.1.13) для приближенных решений. Если оценки типа (3.1.13) выполняются равномерно по параметру дискретизацации, то можно ожидать аналогичного поведения от приближенных решений задачи (4.6.2).

4.6.1. Предварительные результаты о дихотомии. В этом разделе рассмотрим общий аппроксимационный подход для сохранения дихотомических оценок (3.1.13) при аппроксимации траектории $u(\cdot)$ задачи (4.1.5) в окрестности $\mathcal{U}_{E^{\theta}}\left(u^{*} ; \rho\right)$ гиперболической точки равновесия $u^{*}$ некоторыми дискретными траекториями.

Пусть $\mathbb{T}(r)=\{\lambda: \lambda \in \mathbb{C},|\lambda|=r\}, \mathbb{T}=\mathbb{T}(1)$.

Определение 4.6.1. $C_{0}$-Полугруппа $e^{t A}, t \geqslant 0$, определенная на банаховом пространстве $E$, называется гиперболической, если $\sigma\left(e^{t A}\right) \cap \mathbb{T}=\varnothing$ для всех $t>0$. Генератор $A$ называется гиперболическим, если $\sigma(A) \cap i \mathbb{R}=\varnothing$.

Обозначим через $\Upsilon(\mathbb{R} ; E)$ одно из пространств $L^{p}(\mathbb{R} ; E), 1 \leqslant p<\infty, C_{0}(\mathbb{R} ; E)$ или пространство Степанова $S^{p}(\mathbb{R} ; E), 1 \leqslant p<\infty$; будем называть такое пространство пространством Палмера 
(см. [212], где впервые было отмечено свойство фредгольмовости). В банаховом пространстве $\Upsilon(\mathbb{R} ; E)$ рассмотрим линейный дифференциальный оператор

$$
\mathcal{L}=-\frac{d}{d t}+A: D(\mathcal{L}) \subseteq \Upsilon(\mathbb{R} ; E) \rightarrow \Upsilon(\mathbb{R} ; E),
$$

где оператор $A$ порождает $C_{0}$-полугруппу. Предположим, что область определения оператора $\mathcal{L}$ состоит из таких функций $u(\cdot) \in \Upsilon(\mathbb{R} ; E)$, что для некоторой функции $g(\cdot) \in \Upsilon(\mathbb{R} ; E)$ имеем

$$
u(t)=e^{(t-s) A} u(s)-\int_{s}^{t} e^{(t-\eta) A} g(\eta) d \eta, \quad s \leqslant t, \quad t \in \mathbb{R},
$$

и $\mathcal{L} u(\cdot)=g(\cdot)$. Заметим $\left(\right.$ см. [5]), что оператор $\mathcal{L}$ является генератором $C_{0}$-полугруппы $e^{t \mathcal{L}}$ на банаховом пространстве $\Upsilon(\mathbb{R} ; E)$, которая определена для всех $v(\cdot) \in \Upsilon(\mathbb{R} ; E)$ по формуле

$$
\left(e^{t \mathcal{L}} v\right)(s)=e^{t A} v(s-t) \quad \text { для любого } s \in \mathbb{R}, t \geqslant 0 .
$$

Определение 4.6.2. Будем говорить, что $C_{0}$-полугруппа $e^{t A}, t \geqslant 0$, обладает экспоненииальной дихотомией на $\mathbb{R}$ с данными $(M \geqslant 1, \beta>0)$, если существует такой проектор $P: E \rightarrow E$, что

(i) $e^{t A} P=P e^{t A}$ для всех $t \geqslant 0$;

(ii) сужение $\left.e^{t A}\right|_{\mathcal{R}(P)}, t \geqslant 0$, обратимо на $P(E)$ и

$$
\begin{array}{ll}
\left\|e^{-t A} P x\right\| \leqslant M e^{-\beta t}\|P x\|, & t \geqslant 0, \quad x \in E, \\
\left\|e^{t A}(I-P) x\right\| \leqslant M e^{-\beta t}\|(I-P) x\|, & t \geqslant 0, \quad x \in E .
\end{array}
$$

Теорема 4.6.1 (см. [72]). Оператор $\mathcal{L}$ в банаховом пространстве $\Upsilon(\mathbb{R} ; E)$ обратим тогда и только тогда, когда выполнено условие

$$
\sigma\left(e^{1 A}\right) \cap \mathbb{T}=\varnothing .
$$

Если условие (4.6.4) выполняется, то

$$
\left(\mathcal{L}^{-1} f\right)(t)=\int_{-\infty}^{\infty} G(t-s) f(s) d s, \quad t \in \mathbb{R}, \quad f(\cdot) \in \Upsilon(\mathbb{R} ; E),
$$

где функиия Грина имеет вид

$$
G(\eta)=\left\{\begin{array}{rc}
-e^{\eta A} P_{-}, & \eta \geqslant 0 \\
e^{\eta A} P_{+}, & \eta<0
\end{array}\right.
$$

и удовлетворяет оценке

$$
\|G(\eta)\| \leqslant \begin{cases}M_{+} e^{-\gamma_{+} \eta}, & \eta \geqslant 0 \\ M_{-} e^{\gamma_{-} \eta}, & \eta<0\end{cases}
$$

гde

$$
\begin{gathered}
M_{+}=2 M \varkappa(\mathcal{L})\left(1+\frac{1}{2 \varkappa(\mathcal{L})}\right)^{2}, \quad M_{-}=2 M \varkappa(\mathcal{L})\left(1-\frac{1}{2 \varkappa(\mathcal{L})}\right)^{2}, \\
\gamma_{+}=\ln \left(1+\frac{1}{2 \varkappa(\mathcal{L})}\right), \quad \gamma_{-}=-\ln \left(1-\frac{1}{2 \varkappa(\mathcal{L})}\right), \\
\varkappa(\mathcal{L})=1+C(\Upsilon)\left(M+M^{2}\left\|\mathcal{L}^{-1}\right\|\right) .
\end{gathered}
$$

Заметим, что $C(\Upsilon)=1$, если $\Upsilon(\mathbb{R} ; E)=L^{\infty}(\mathbb{R}, E)$ или $\Upsilon(\mathbb{R} ; E)=C_{0}(\mathbb{R}, E)$, и $C(\Upsilon)=2^{1-1 / p}$, если $\Upsilon(\mathbb{R} ; E)=L^{p}(\mathbb{R}, E)$ или $\Upsilon(\mathbb{R} ; E)=S^{p}(\mathbb{R}, E), p \in[1, \infty)$. 
Обозначим через $\Upsilon(\mathbb{Z} ; E)$, где $\mathbb{Z}=\{\ldots,-2,-1,0,1,2, \ldots\}$, банахово пространство $E$-значных последовательностей с соответствующей дискретной нормой, согласованной с нормой простран-

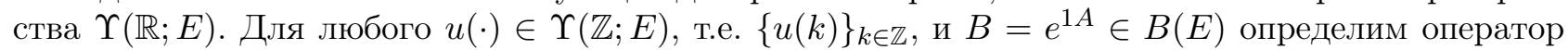
$\mathcal{B}: D(\mathcal{B}) \subseteq l^{p}(\mathbb{Z} ; E) \rightarrow l^{p}(\mathbb{Z} ; E)$ по формуле

$$
(\mathcal{B} u)(k)=B u(k-1), \quad k \in \mathbb{Z}, \quad u(\cdot) \in l^{p}(\mathbb{Z} ; E) .
$$

Определим также оператор $\mathcal{D}=I-\mathcal{B}: D(\mathcal{D})=D(\mathcal{B}) \subseteq l^{p}(\mathbb{Z} ; E) \rightarrow l^{p}(\mathbb{Z} ; E)$ следующим образом:

$$
(\mathcal{D} u)(k)=u(k)-B u(k-1), \quad u(\cdot) \in D(\mathcal{B}), \quad k \in \mathbb{Z} .
$$

Предложение 4.6.1 (см. [72]). Пусть оператор

$$
\mathcal{L}=-\frac{d}{d t}+A: D(\mathcal{L}) \subseteq \Upsilon(\mathbb{R} ; E) \rightarrow \Upsilon(\mathbb{R} ; E)
$$

является обратимым. Тогда оператор $\mathcal{D}: D(\mathcal{D}) \subseteq \Upsilon(\mathbb{Z} ; E) \rightarrow \Upsilon(\mathbb{Z} ; E)$ также обратим и

$$
\left\|\mathcal{D}^{-1}\right\| \leqslant 1+C(\Upsilon)\left(M+M^{2}\left\|\mathcal{L}^{-1}\right\|\right)
$$

Обратно, если $\mathcal{D}$ обратим, то $\mathcal{L}: D(\mathcal{L}) \subseteq \Upsilon(\mathbb{R} ; E) \rightarrow \Upsilon(\mathbb{R} ; E)$ также обратим и

$$
\left\|\mathcal{L}^{-1}\right\| \leqslant C(\Upsilon)\left(M+M^{2}\left\|\mathcal{D}^{-1}\right\|\right)
$$

Теорема 4.6.2 (см. [6, 8]). Разностный оператор

$$
(\mathcal{D} u)(k)=u(k)-B u(k-1), \quad k \in \mathbb{N}, \quad u(\cdot) \in l^{p}(\mathbb{R} ; E), \quad 1 \leqslant p \leqslant \infty,
$$

обратим тогда и только тогда, когда

$$
\sigma(B) \cap \mathbb{T}=\varnothing .
$$

Если условие (4.6.7) выполнено, то обратный оператор имеет вид

$$
\left(\mathcal{D}^{-1} v\right)(k)=\sum_{m \in \mathbb{Z}} \Gamma(k-m) v(m),
$$

где $v(\cdot) \in l^{p}(\mathbb{Z} ; E), 1 \leqslant p \leqslant \infty$, и функция $\Gamma(\cdot): \mathbb{Z} \rightarrow B(E)$ определена формулой

$$
\Gamma(k)=\left\{\begin{array}{cl}
B^{k}(I-P), & k \geqslant 0 ; \\
-B_{0}^{-k} P, & k \leqslant-1,
\end{array}\right.
$$

где $B_{0}$ - сужение $B$ на $\mathcal{R}(P)$.

Определение 4.6.3. Будем говорить, что оператор $B \in B(E)$ имеет экспоненциалъную дискретную дихотомию с данными $(M, r, P)$, если $P \in B(E)$ - проектор в $E$ и $M, r$-такие константы, $0 \leqslant r<1$, что выполнены следующие свойства:

(i) $B^{k} P=P B^{k}$ для всех $k \in \mathbb{N}$;

(ii) $\left\|B^{k}(I-P)\right\| \leqslant M r^{k}$ для всех $k \in \mathbb{N}$;

(iii) $\hat{B}:=\left.B\right|_{\mathcal{R}(P)}: \mathcal{R}(P) \mapsto \mathcal{R}(P)$ - гомеоморфизм, удовлетворяющий оценке

$$
\left\|\hat{B}^{-k} P\right\| \leqslant M r^{k}, \quad k \in \mathbb{N} .
$$

Следующий результат касается связи констант в экспоненциальной дискретной дихотомии при оценке резольвенты $(\lambda I-B)^{-1}$ при $\lambda \in \mathbb{T}$.

Теорема 4.6.3 (см. [214]). При $B \in B(E)$ следуюшие условия эквивалентны:

(i) $\lambda \in \rho(B) \partial л я в$ всех $\lambda \in \mathbb{T} u$

$$
\left\|(\lambda I-B)^{-1}\right\| \leqslant \beta<\infty \quad \forall \lambda \in \mathbb{T}
$$

(ii) В имеет экспоненииальную дихотомию с данными $(M, r, P)$. 
Точнее, из (i) следует (ii) c

$$
M=\frac{2 \beta^{2}}{\beta-1}, \quad r=1-\frac{1}{2 \beta} .
$$

Обратно, из (ii) следует (i) $c \beta=M \frac{1+r}{1-r}$.

Доказательство. Пусть выполнено (i); без ограничения общности будем считать, что $\beta>1$. Для $z \in \mathbb{C}, z \neq 0$ имеем

$$
\left|z-\frac{z}{|z|}\right|=|1-| z||
$$

Таким образом, если $|1-| z|| \beta<1$, классическая оценка возмущения показывает, что $z \in \rho(B)$ и

$$
\left\|(z I-B)^{-1}\right\| \leqslant \frac{\beta}{1-\beta|1-| z||} .
$$

Определим $P$ как проектор Рисса, определенный формулой

$$
I-P=\frac{1}{2 \pi i} \int_{|z|=1}(z I-B)^{-1} d z .
$$

Поскольку $B$ коммутирует с резольвентой, выполнено условие (i) определения 4.6.3. Далее, используя (4.6.11) и теорему Коши, можем сдвинуть контур:

$$
I-P=\frac{1}{2 \pi i} \int_{|z|=r}(z I-B)^{-1} d z, \quad \text { если }|1-r|<\frac{1}{\beta} .
$$

Утверждается, что

$$
B^{k}(I-P)=\frac{1}{2 \pi i} \int_{|z|=r} z^{k}(z I-B)^{-1} d z, \quad \text { если } \quad|1-r|<\frac{1}{\beta} .
$$

Для $k=0$ это вытекает из (4.6.13). Если (4.6.14) имеет место для некоторого $k$, то получим

$$
B^{k+1} P=\frac{1}{2 \pi i} \int_{|z|=r}(B-z I+z I) z^{k}(z I-B)^{-1} d z=\frac{1}{2 \pi i} \int_{|z|=r} z^{k+1}(z I-B)^{-1} d z-\frac{1}{2 \pi i} \int_{|z|=r} z^{k} d z
$$

и, таким образом, утверждение имеет место для $k+1$. Из уравнений (4.6.14) и (4.6.11) непосредственно получаем, во-первых, дихотомическую оценку для $1-1 / \beta<r \leqslant 1$ :

$$
\left\|B^{k} P\right\| \leqslant \frac{1}{2 \pi} 2 \pi r r^{k} \frac{\beta}{1-\beta(1-r)}=\frac{\beta r^{k+1}}{1-\beta(1-r)} \quad \text { для } k \geqslant 0 .
$$

Для второй дихотомической оценки мы используем резольвенту уравнения

$$
(z I-B)^{-1}=\frac{1}{z} I+z^{-1} B(z I-B)^{-1} .
$$

При $|1-r|<1 / \beta$ уравнения (4.6.13) и (4.6.16) приводят к соотношению

$$
I-P=\frac{1}{2 \pi i} \int_{|z|=1}\left(\frac{1}{z} I-(z I-B)^{-1}\right) d z=-\frac{1}{2 \pi i} \int_{|z|=r} \frac{1}{z} B(z I-B)^{-1} d z .
$$

Отсюда ясно, что для $k=1$ имеет место следующее равенство:

$$
I-P=-B^{k} \frac{1}{2 \pi i} \int_{|z|=r} z^{-k}(z I-B)^{-1} d z \quad \text { для } k \geqslant 1 .
$$

Если (4.6.17) известно для некоторого $k$, то, используя (4.6.16), найдем

$$
I-P=-B^{k} \frac{1}{2 \pi i} \int_{|z|=r}\left(z^{-(k+1)}+z^{-(k+1)} B(z I-B)^{-1}\right) d z=-B^{k+1} \frac{1}{2 \pi i} \int_{|z|=r} z^{-(k+1)}(z I-B)^{-1} d z .
$$


Применим (4.6.17) к $x \in E$ и воспользуемся тем фактом, что $I-P$ коммутирует с $B$, и оценкой (4.6.11):

$$
\|(I-P) x\|=\left\|\frac{1}{2 \pi i} \int_{|z|=r} z^{-k}(z I-B)^{-1} d z B^{k}(I-P) x\right\| \leqslant \frac{r^{-k}}{2 \pi} 2 \pi r \frac{\beta}{1-\beta(r-1)}\left\|B^{k}(I-P) x\right\| .
$$

Окончательно имеем для всех $k \geqslant 1,1 \leqslant r<1+1 / \beta, u \in E$ :

$$
\|(I-P) u\| \leqslant \frac{\beta r^{-k+1}}{1-\beta(r-1)}\left\|B^{k}(I-P) u\right\|
$$

При $k=1$ эта оценка показывает, что оператор $\hat{B}=\left.B\right|_{\mathcal{N}(P)}: \mathcal{N}(P) \mapsto \mathcal{N}(P)$ взаимно однозначен и имеет ограниченный обратный. Чтобы показать, что $\hat{B}$ сюръективен, возьмем $f \in \mathcal{N}(P)$ и положим

$$
v=-\frac{1}{2 \pi i} \int_{|z|=1} z^{-1}(z I-B)^{-1} f d z
$$

Из этого уравнения имеем $(I-P) v=0$; применяя (4.6.16), находим

$$
B v=\frac{1}{2 \pi i} \int_{|z|=1}\left(z^{-1} I-(z I-B)^{-1}\right) f d z=(I-P) f=f .
$$

Следовательно, $\hat{B}$ - линейный гомеоморфизм на $\mathcal{N}(P)$, удовлетворяющий оценке

$$
\left\|\hat{B}^{-k}(I-P) u\right\| \leqslant \frac{\beta r^{-k+1}}{1-\beta(r-1)}\|(I-P) u\| \quad \text { при } \quad 1 \leqslant r<1+\frac{1}{\beta} .
$$

Это доказывает экспоненциальную дихотомию.

Чтобы получить значения констант, положим $r=1-1 /(2 \beta)$ в (4.6.15) и получим оценку $(2 \beta-1) r^{k}$. Чтобы получить такую же скорость в обратном направлении, применим (4.6.19), взяв

$$
r=\left(1-\frac{1}{2 \beta}\right)^{-1}<1+\frac{1}{\beta}
$$

В (4.6.19) тогда найдем верхнюю границу $M r^{-k}$ с константой $M=\left(2 \beta^{2}\right) /(\beta-1)$. Поскольку $M>2 \beta-1$, получаем требуемое утверждение.

Теперь предположим экспоненциальную дихотомию и докажем условие (i). При $|\lambda|=1$ уравнение $(\lambda I-B) u=f$ эквивалентно системе

$$
(\lambda I-B P) P u=P f, \quad(\lambda I-\hat{B})(I-P) u=(I-P) f,
$$

которую можно переписать в виде

$$
\left(I-\lambda^{-1} B P\right) P u=\lambda^{-1} P f, \quad\left(I-\lambda \hat{B}^{-1}\right)(I-P) u=-\hat{B}^{-1}(I-P) f .
$$

Оба уравнения имеют единственное решение, задаваемое геометрическим рядом

$$
\begin{gathered}
P u=\sum_{k=0}^{\infty} \lambda^{-(k+1)} B^{k} P f \\
(I-P) u=-\sum_{k=0}^{\infty} \hat{B}^{-(k+1)} \lambda^{k}(I-P) f .
\end{gathered}
$$

Из экспоненциальной дихотомии тогда получаем оценки

$$
\|P u\| \leqslant \frac{M}{1-r}\|f\|, \quad\|(I-P) u\| \leqslant M \frac{r}{1-r}\|f\| .
$$

Согласно неравенству треугольника получим условие (i) с $\beta=M \frac{1+r}{1-r}$. 
4.6.2. Дискретизация дихотомических полугрупп. Рассмотрим случай, когда резольвента оператора $A$ не обязательно является компактной.

Теорема 4.6.4 (см. [86]). Пусть операторы $\lambda I_{n}-B_{n} \in B\left(E_{n}\right)$ фредгольмовы $u$ ind $\left(\lambda I_{n}-B_{n}\right)=$ 0 для всех $\lambda \in \mathbb{T}, n \in \mathbb{N}$. Предположим также, что $B \in B(E)$ обладает свойством $\mathbb{T} \cap \sigma(B)=\varnothing$ и $\lambda I_{n}-B_{n} \stackrel{\mathcal{P P}}{\longrightarrow} \lambda I-B$ собственно для любого $\lambda \in \mathbb{T}$. Тогда $\lambda I_{n}-B_{n} \stackrel{\mathcal{P P}}{\longrightarrow} \lambda I-B$ устойчиво для любого $\lambda \in \mathbb{T} u$

$$
\sup _{\lambda \in \mathbb{T}}\left\|\left(\lambda I_{n}-B_{n}\right)^{-1}\right\|<\infty .
$$

Доказательство. Предположим, что найдутся некоторые последовательности $\left\{\lambda_{n}\right\}, \lambda_{n} \in \mathbb{T}$, и $\left\{x_{n}\right\}, x_{n} \in E_{n}$, для которых $\left\|x_{n}\right\|=1$ и $\left(\lambda_{n} I_{n}-B_{n}\right) x_{n} \stackrel{\mathcal{P}}{\longrightarrow} 0$ при $n \rightarrow \infty$. Поскольку $\mathbb{T}$ компактно, можно найти такое подмножество $\mathbb{N}^{\prime} \subset \mathbb{N}$, что $\lambda_{n} \rightarrow \lambda_{0} \in \mathbb{T}$ при $n \in \mathbb{N}^{\prime}$. При этом

$$
\lambda_{0} I_{n}-B_{n} \stackrel{\mathcal{P P}}{\longrightarrow} \lambda_{0} I-B \quad \text { собственно }
$$

для такого $\lambda_{0} \in \mathbb{T}$ и

$$
\left(\lambda_{0} I_{n}-B_{n}\right) x_{n}=\left(\lambda_{0} I_{n}-\lambda_{n} I_{n}\right) x_{n}+\left(\lambda_{n} I_{n}-B_{n}\right) x_{n} \stackrel{\mathcal{P}}{\longrightarrow} 0 \quad \text { при } n \in \mathbb{N}^{\prime} .
$$

Следовательно, найдется такое подмножество $\mathbb{N}^{\prime \prime} \subset \mathbb{N}^{\prime}$, что $x_{n} \stackrel{\mathcal{P}}{\longrightarrow} x_{0} \neq 0$ при $n \in \mathbb{N}^{\prime \prime}$. Но в таком случае

$$
\left(\lambda_{0} I_{n}-B_{n}\right) x_{n} \stackrel{\mathcal{P}}{\longrightarrow}\left(\lambda_{0} I-B\right) x_{0}=0 \quad \text { для } n \in \mathbb{N}^{\prime \prime},
$$

что противоречит предположению $\mathbb{T} \cap \sigma(B)=\varnothing$.

Определение 4.6.4. Будем говорить, что операторы $B_{n} \in B\left(E_{n}\right)$ имеют равномерную экспоненииальную дискретную дихотомию с данными $\left(M, r, P_{n}\right)$, если $P_{n} \in B\left(E_{n}\right)$ - проектор в $E_{n}$ и $M, r$ - такие константы, $0 \leqslant r<1$, что выполнены следующие условия:

(i) $B_{n}^{k} P_{n}=P_{n} B_{n}^{k}$ и $\left\|P_{n}\right\| \leqslant$ const для всех $k, n \in \mathbb{N}$;

(ii) $\left\|B_{n}^{k}\left(I_{n}-P_{n}\right)\right\| \leqslant M r^{k}$ для всех $k, n \in \mathbb{N}$;

(iii) $\hat{B}_{n}:=\left.B_{n}\right|_{\mathcal{R}\left(P_{n}\right)}: \mathcal{R}\left(P_{n}\right) \mapsto \mathcal{R}\left(P_{n}\right)$ - гомеоморфизм, удовлетворяющий оценке

$$
\left\|{\hat{B_{n}}}^{-k} P_{n}\right\| \leqslant M r^{k}, \quad k, n \in \mathbb{N} .
$$

Теорема 4.6.5 (см. [86]). Следующие условия эквивалентны:

(i) $\lambda I_{n}-B_{n} \stackrel{\mathcal{P P}}{\longrightarrow} \lambda I-B$ устойчиво и $\lambda \in \rho(B)$ для любого $\lambda \in \mathbb{T}$;

(ii) оператор $\mathcal{D}=I-\mathcal{B}$ обратим и $\mathcal{D}_{n} \stackrel{\mathcal{P P}}{\longrightarrow} \mathcal{D}$ устойчиво, где $(\mathcal{B} u)(k)=B u(k-1), k \in \mathbb{N}$;

(iii) $B_{n} \stackrel{\mathcal{P P}}{\longrightarrow} B$, оператор $\lambda I-B$ обратим для любого $\lambda \in \mathbb{T}$ и операторы $B_{n}$ имеют экспоненииальную дискретную дихотомию с данными $\left(M, r, P_{n}\right)$ равномерно по $n \in \mathbb{N}$.

Доказательство. Эквивалентность (i) муле (4.6.12) получаем из (i), что $P_{n} \stackrel{\mathcal{P P}}{\longrightarrow} P$ и $\left\|P_{n}\right\| \leqslant$ const. По теореме 4.6.4 имеем

$$
\sup _{\lambda \in \mathbb{T}}\left\|\left(\lambda I_{n}-B_{n}\right)^{-1}\right\|<\infty
$$

и по теореме 4.6.3(ii) получаем (iii). Обратно, из условия (iii) следует в силу теоремы 4.6.3(i), что $\lambda I_{n}-B_{n} \stackrel{\mathcal{P P}}{\longrightarrow} \lambda I-B$ устойчиво для всех $\lambda \in \mathbb{T}$.

Чтобы доказать импликацию $(\mathrm{ii}) \Longrightarrow(\mathrm{i})$, заметим, что (ii) означает, что для любого $u(\cdot) \in$ $l^{p}(\mathbb{Z} ; E)$ имеем

$$
\sum_{k=-\infty}^{\infty}\left\|p_{n} u(k)-B_{n} p_{n} u(k-1)-p_{n} u(k)+p_{n} B u(k-1)\right\|_{E_{n}}^{p} \rightarrow 0 \quad \text { при } n \rightarrow \infty,
$$


т.е. $B_{n} \stackrel{\mathcal{P P}}{\longrightarrow} B$. Предположим теперь, что $I_{n}-B_{n}$ не является равномерно обратимым в $l^{\infty}\left(\mathbb{Z} ; E_{n}\right)$, т.е. для некоторой последовательности $\left\|x_{n}\right\|=1$ имеем

$$
\left(\lambda_{0} I_{n}-B_{n}\right) x_{n} \stackrel{\mathcal{P}}{\longrightarrow} 0 \quad \text { при } n \rightarrow \infty \text { для } \lambda_{0}=1 \in \mathbb{T} .
$$

Это означает, что для стационарной последовательности $u_{n}(k)=x_{n}, k \in \mathbb{Z}, n \in \mathbb{T}$, имеем

$$
\left(\mathcal{D}_{n} u_{n}\right)(k)=u_{n}(k)-B_{n} u_{n}(k-1)=x_{n}-B_{n} x_{n} \stackrel{\mathcal{P}}{\longrightarrow} 0 \quad \text { для любого } k \in \mathbb{N} \text { при } n \rightarrow \infty .
$$

Но $\mathcal{D}_{n} \stackrel{\mathcal{P} \mathcal{P}}{\longrightarrow} \mathcal{D}$ устойчиво, т.е.

$$
\left\|\mathcal{D}_{n} u_{n}\right\|_{l^{\infty}\left(\mathbb{Z} ; E_{n}\right)} \geqslant \gamma\left\|u_{n}\right\|_{l^{\infty}\left(\mathbb{Z} ; E_{n}\right)}
$$

что противоречит условию

$$
\left(\lambda_{0} I_{n}-B_{n}\right) x_{n} \stackrel{\mathcal{P}}{\longrightarrow} 0 \quad \text { при } n \rightarrow \infty .
$$

Теперь покажем, что $\mathcal{R}\left(\lambda_{0} I_{n}-B_{n}\right)=E_{n}$. Для любого $y_{n} \in E_{n},\left\|y_{n}\right\|=1$, рассмотрим $v_{n}(k)=y_{n}$, $k \in \mathbb{Z}, n \in \mathbb{N}$. Решение уравнения $\mathcal{D}_{n} u_{n}=v_{n}$-это последовательность $u_{n}(k)$, которая также стационарна, т.е. $\left(\lambda_{0} I_{n}-B_{n}\right) x_{n}=y_{n}$, где $x_{n}=u_{n}(k), k \in \mathbb{Z}, n \in \mathbb{N}$. Чтобы доказать импликацию (i) $\Longrightarrow($ ii), заметим, что

$$
\left\|\mathcal{D}_{n}\right\|_{B\left(l^{p}\left(\mathbb{Z} ; E_{n}\right)\right)} \leqslant \text { const, } \quad n \in \mathbb{N} .
$$

Теперь для любого $u(\cdot) \in l^{p}(\mathbb{Z} ; E)$ и любого $\epsilon>0$ можно найти такое $K \in \mathbb{N}$, что

$$
\left(\sum_{k=K}^{\infty}+\sum_{k=-K}^{-\infty}\right)\|u(k)\|^{p} \leqslant \epsilon
$$

Кроме того,

$$
\sum_{k=-K}^{K}\left\|p_{n} u(k)-B_{n} p_{n} u(k-1)-p_{n} u(k)+p_{n} B u(k-1)\right\|_{E_{n}}^{p} \rightarrow 0 \quad \text { при } n \rightarrow \infty,
$$

поскольку $B_{n} \stackrel{\mathcal{P P}}{\longrightarrow} B$. Итак, имеем $\mathcal{D}_{n} \stackrel{\mathcal{P} \mathcal{P}}{\longrightarrow} \mathcal{D}$. Сходимость $\mathcal{D}_{n}^{-1} \stackrel{\mathcal{P} \mathcal{P}}{\longrightarrow} \mathcal{D}^{-1}$ следует из формулы (4.6.8). Теорема доказана.

\section{7. ОБЩАЯ ДИХОТОМИЯ ПРИ ПОЛУДИСКРЕТИЗАЦИИ}

В разделе 3.1.2 было показано, что существование изолированного гиперболического равновесия $u^{*}=-A^{-1} f\left(u^{*}\right)$ следует из компактности оператора $A^{-1} f(\cdot)$ и гладкости функции $f(\cdot)$. В случае уплотняющего оператора $A^{-1} f(\cdot)$ с константой $q<1$ существование изолированного гиперболического равновесия $u^{*}=-A^{-1} f\left(u^{*}\right)$ вытекает из результатов [34, Sec. 3.3.5] в силу дифференцируемости функции $f(\cdot)$. Уплотняющее свойство оператора $A^{-1} f(\cdot)$ можно вывести, например, как следствие $[46,(9.3)]$. Если операторы $A_{n}^{-1} f_{n}(\cdot)$ также являются уплотняющими с константой $q<1$, то из ограниченности последовательности $\left\|x_{n}\right\|$ и $\mathcal{P}$-компактности $\left\{x_{n} I_{n}-\lambda A_{n}^{-1} f_{n}\left(x_{n}\right)\right\}$ для любого $0<\lambda \leqslant 1$ следует, что последовательность $\left\{x_{n}\right\}$ является $\mathcal{P}$-компактной. Таким образом, все условия теоремы 4 из [259] выполнены и, следовательно, точки равновесия $\left\{u_{n}^{*}\right\}$ для (4.2.9) существуют и обладают свойством $u_{n}^{*} \stackrel{\mathcal{P}}{\longrightarrow} u^{*}$.

Теперь мы можем сформулировать основной результат о равномерных по индексу $n$ оценках для членов дискретных решений задачи (4.2.17).

Теорема 4.7.1 (см. [86]). Пусть операторы $A_{n}, A$ являются генераторами аналитических $C_{0}$-полугрупп и выполнено условие $\left(\mathrm{B}_{1}\right)$. Предположим такље, что полугруппа $e^{t A_{u^{*}}}$ является гиперболической,

$$
\sigma\left(A_{u^{*}}\right) \cap\{\lambda: \operatorname{Re} \lambda \geqslant 0\} \subseteq P \sigma\left(A_{u^{*}}\right), \quad \operatorname{dim} P(\sigma+)<\infty,
$$


и для $\rho>0$, удовлетворяющего условию $\{\lambda:-\rho \leqslant \operatorname{Re} \lambda \leqslant \rho\} \subset \rho\left(A_{u^{*}}\right), \lambda I_{n}-A_{u_{n}^{*}, n}-\oint$ фрдгольмовы операторы индекса 0 и операторы $\lambda I_{n}-A_{u_{n}^{*}, n}, \lambda I-A_{u^{*}}$ собственно согласованы для любого $\operatorname{Re} \lambda \geqslant-\rho$. Тогда $P_{n}\left(\sigma_{n}^{+}\right) \stackrel{\mathcal{P P}}{\longrightarrow} P\left(\sigma^{+}\right)$компактно и

$$
\begin{cases}\left\|e^{t A_{u_{n}^{*}, n}}\left(I_{n}-P_{n}\left(\sigma_{n}^{+}\right)\right)\right\|_{E_{n}} \leqslant M_{2} e^{-\gamma t}, & t \geqslant 0, \\ \left\|e^{t A_{u_{n}^{*}, n}} P_{n}\left(\sigma_{n}^{+}\right)\right\|_{E_{n}} \leqslant M_{2} e^{\gamma t}, & t \leqslant 0,\end{cases}
$$

әде $\gamma>0$.

Доказательство. Из условия $\left(\mathrm{B}_{1}\right)$ следует, что

$$
\left(\lambda I_{n}-A_{u_{n}^{*}, n}\right)^{-1} \stackrel{\mathcal{P P}}{\longrightarrow}\left(\lambda I-A_{u^{*}}\right)^{-1}
$$

при $-\rho \leqslant \operatorname{Re} \lambda \leqslant \rho$ и достаточно большом $|\lambda|$. Для других значений $\lambda$, удовлетворяющих условию $-\rho \leqslant \operatorname{Re} \lambda \leqslant \rho$, сходимость (4.7.2) следует из аналога теоремы 3.2.2 для замкнутых операторов. Факт компактной сходимости $P_{n}\left(\sigma_{n}^{+}\right) \stackrel{\mathcal{P} \mathcal{P}}{\longrightarrow} P\left(\sigma^{+}\right)$получим, как в [26], а оценку (4.7.1) - как в [79, 89]. Теорема 4.7 .1 доказана.

Замечание 4.7.1. Разумеется теорема 4.7.1 имеет место для любого оператора $A$, порождающего аналитическую гиперболическую $C_{0}$-полугруппу и удовлетворяющего условиям $\sigma(A) \cap\{\lambda$ : $\operatorname{Re} \lambda \geqslant 0\} \subseteq P \sigma(A)$ и $\operatorname{dim} P(\sigma+)<\infty$, а также с соответствующими условиям на аппроксимацию операторов. Структура оператора типа $A+f_{u}^{\prime}\left(u^{*}\right)$ не является необходимой.

Теперь теорему 4.7.1 легко получить в виде следующего утверждения.

Следствие 4.7.1. Пусть операторы $A_{n}, A$ являются генераторами аналитических $C_{0}$-полугрупп, причем выполнено условие $\left(\mathrm{B}_{1}\right)$. Предположим также, что $C_{0}$-полугруппа $e^{\text {tA } \text { u}^{*}}$ является гиперболической

$$
\sigma\left(A_{u^{*}}\right) \cap\{\lambda: \operatorname{Re} \lambda \geqslant 0\}=P \sigma\left(A_{u^{*}}\right), \quad \operatorname{dim} P(\sigma+)<\infty .
$$

Далее, пусть $\Delta_{c c}\left(A_{n}, A\right) \neq \varnothing$ и резольвенты операторов $A_{n}$ и $А$ являются компактными операторами. Тогда имеет место (4.7.1).

Доказательство. Положим $B_{n}=e^{1 A_{u_{n}^{*}, n}}$ и $B=e^{1 A_{u^{*}}}$. Известно (см. [26]), что условие $\Delta_{c c} \neq \varnothing$ эквивалентно компактной сходимости $B_{n} \stackrel{\mathcal{P P}}{\longrightarrow} B$. Тогда условие (i) теоремы 4.6 .5 выполнено, и мы получаем дискретную дихотомию для $B_{n}$. С другой стороны, поскольку $B_{n}=e^{1 A_{u_{n}^{*}, n}}$, это означает, согласно теореме 4.6.3 и предложению 4.6 .1 , что операторы $\mathcal{L}_{n}=-\frac{d}{d t}+A_{u_{n}^{*}, n}$ обратимы и, следовательно, благодаря теореме 4.6.1 получаем оценки (4.7.1).

4.7.1. Дихотомия для уплотняющих операторов при полудискретизации. Будем использовать те же обозначения, что в теореме 3.2.3.

Определение 4.7.1. Будем говорить, что операторы $B_{n} \in B\left(E_{n}\right)$ являются совместно уплотняющими с константой $q>0$ по отношению к мере $\mu(\cdot)$, если для любой ограниченной последовательности $\left\{x_{n}\right\}, x_{n} \in E_{n}$, имеем

$$
\mu\left(\left\{B_{n} x_{n}\right\}\right) \leqslant q \mu\left(\left\{x_{n}\right\}\right) .
$$

Известно (см. [34, с. 82]), что вне замкнутого круга радиуса $q$ с центром в нуле все операторы $B_{n}$ имеют лишь изолированные точки спектра, каждая из которых может быть только собственным значением конечной корневой кратности.

Предложение 4.7 .1 (см. [214]). Пусть $B_{n} \stackrel{\mathcal{P P}}{\longrightarrow} B$ для $B_{n} \in B\left(E_{n}\right), B \in B(E) u$

$$
\mu\left(\left\{B_{n} x_{n}\right\}\right) \leqslant q \mu\left(\left\{x_{n}\right\}\right)
$$


для любой ограниченной последовательности $\left\{x_{n}\right\}, x_{n} \in E_{n}$. Предположим, что $\sigma(B) \cap \Psi=\varnothing$, где $\Psi \subset \mathbb{C} \backslash\{\lambda:|\lambda| \leqslant q\}$-ограниченное замкнутое множество и $\sigma(B) \backslash\{\lambda:|\lambda| \leqslant q\}$ состоит только из точек дискретного спектра. Тогда найдется такая константа $C>0$, что $\left\|\left(\lambda I_{n}-B_{n}\right)^{-1}\right\| \leqslant C, \lambda \in \Psi, n \in \mathbb{N}$.

Доказательство. Любая точка $\lambda \in \Psi$ принадлежит множеству $P \sigma\left(B_{n}\right) \cup \rho\left(B_{n}\right)$. Это означает, что для последовательности $\left\|x_{n}\right\|=1, x_{n} \in E_{n}$, возможны два случая:

$$
\left(\lambda I_{n}-B_{n}\right) x_{n}=0 \quad \text { или } \quad\left\|\left(\lambda I_{n}-B_{n}\right) x_{n}\right\| \geqslant \gamma_{\lambda, n}\left\|x_{n}\right\|
$$

где $\gamma_{\lambda, n}>0$ и $\lambda \in \Psi$. Покажем, что на самом деле

$$
\left\|\left(\lambda I_{n}-B_{n}\right) x_{n}\right\| \geqslant \gamma_{\Psi}\left\|x_{n}\right\|, \quad \lambda \in \Psi .
$$

Предположим, напротив, что найдутся такие последовательности $\left\{\lambda_{n}\right\}, \lambda_{n} \in \Psi$, и $\left\{x_{n}\right\},\left\|x_{n}\right\|=1$, что

$$
\left(\lambda_{n} I_{n}-B_{n}\right) x_{n} \stackrel{\mathcal{P}}{\longrightarrow} 0 \quad \text { при } n \in \mathbb{N} .
$$

Тогда $\lambda_{n} \rightarrow \lambda_{0} \in \Psi, n \in \mathbb{N}^{\prime} \subseteq \mathbb{N}$. Для $\tilde{r}=\inf \{|\xi|: \xi \in \Psi\}$ имеем

$$
\mu\left(\left\{x_{n}\right\}\right) \leqslant \frac{\left|\lambda_{n}\right|}{\tilde{r}} \mu\left(\left\{x_{n}\right\}\right) \leqslant \frac{\mu\left(\left\{B_{n} x_{n}\right\}\right)}{\tilde{r}} \leqslant \frac{q}{\tilde{r}} \mu\left(\left\{x_{n}\right\}\right)
$$

что означает, в силу неравенства $q / \tilde{r}<1$, что $\mu\left(\left\{x_{n}\right\}\right)=0$, т.е. последовательность $\left\{x_{n}\right\}$ является $\mathcal{P}$-компактной. Тогда

$$
\begin{gathered}
x_{n} \stackrel{\mathcal{P}}{\longrightarrow} x_{0}, \quad n \in \mathbb{N}^{\prime \prime} \subseteq \mathbb{N}^{\prime}, \\
B_{n} x_{n} \stackrel{\mathcal{P}}{\longrightarrow} B x_{0}, \quad \lambda_{n} x_{n} \stackrel{\mathcal{P}}{\longrightarrow} \lambda_{0} x_{0}, \quad n \in \mathbb{N}^{\prime \prime},
\end{gathered}
$$

т.е. $\lambda_{0} x_{0}=B x_{0}$ при $\left\|x_{0}\right\|=1$, что противоречит предположению $\sigma(B) \cap \Psi=\varnothing$. Предложение доказано.

Обозначим через $\nu(\cdot)$ меру некомпактности на $E$.

Предложение 4.7 .2 (см. [214]). Пусть $B_{n} \stackrel{\mathcal{P P}}{\longrightarrow} B u \mu\left(\left\{B_{n} x_{n}\right\}\right) \leqslant q \mu\left(\left\{x_{n}\right\}\right)$, для любой ограниченной последовательности $\left\{x_{n}\right\}, x_{n} \in E_{n}, u \nu\left(\left\{B x^{n}\right\}\right) \leqslant q \nu\left(\left\{x^{n}\right\}\right)$ для любой ограниченной последовательности $\left\{x^{n}\right\}, x^{n} \in E$, а также $q<1$. Тогда любое число $\lambda_{0} \in \sigma(B),\left|\lambda_{0}\right|>q$, является изолированным собственным значением, причем проектор $P\left(\lambda_{0}\right)$ конечномерен, и найдутся такие последовательность $\left\{\lambda_{n}\right\}, \lambda_{n} \in \sigma\left(B_{n}\right)$, и последовательность проекторов $P_{n}\left(\lambda_{0}\right) \in B\left(E_{n}\right)$, что $\lambda_{n} \rightarrow \lambda_{0}$ и $P_{n}\left(\lambda_{0}\right) \stackrel{\mathcal{P P}}{\longrightarrow} P\left(\lambda_{0}\right)$ компактно.

Доказательство. Во-первых, заметим, что для $\Gamma_{r}=\left\{\lambda:\left|\lambda-\lambda_{0}\right|=r\right\} \subset \mathbb{C} \backslash\{\lambda:|\lambda| \leqslant q\}$, где $r$ можно выбрать достаточно малым, имеем согласно предложению 4.7.1

$$
\left(\lambda I_{n}-B_{n}\right)^{-1} \stackrel{\mathcal{P P}}{\longrightarrow}(\lambda I-B)^{-1} \quad \text { для } \lambda \in \Gamma_{r}, n \in \mathbb{N} .
$$

Следовательно, $P_{n}\left(\lambda_{0}\right) \stackrel{\mathcal{P P}}{\longrightarrow} P\left(\lambda_{0}\right)$. Чтобы доказать компактность сходимости этих проекторов, заметим, что

$$
\mu\left(\left\{\left(\lambda_{0} I_{n}-B_{n}\right) x_{n}\right\}\right) \geqslant\left|\lambda_{0}\right| \mu\left(\left\{x_{n}\right\}\right)-\mu\left(\left\{B_{n} x_{n}\right\}\right) \geqslant\left|\lambda_{0}\right| \mu\left(\left\{x_{n}\right\}\right)-q \mu\left(\left\{x_{n}\right\}\right) \geqslant \gamma \mu\left(\left\{x_{n}\right\}\right),
$$

где $\gamma=\left|\lambda_{0}\right|-q>0$. Это означает, что

$$
\mu\left(B\left\{\left(\lambda_{0} I_{n}-B_{n}\right)^{k} x_{n}\right\}\right) \geqslant \gamma^{k} \mu\left(\left\{x_{n}\right\}\right) \quad \text { для всех } k \in \mathbb{N} .
$$

Далее,

$$
\left(\lambda_{0} I_{n}-B_{n}\right)^{k} P_{n}\left(\lambda_{0}\right) x_{n}=\frac{1}{2 \pi i} \int_{\Gamma_{r}}\left(\lambda_{0}-\lambda\right)^{k}\left(\lambda I_{n}-B_{n}\right)^{-1} x_{n} d \lambda
$$


Используя (4.7.3), из этого представления находим

$$
\gamma^{k} \mu\left(\left\{P_{n}\left(\lambda_{0}\right) x_{n}\right\}\right) \leqslant\left\|\left(\lambda_{0} I_{n}-B_{n}\right)^{k} P_{n}\left(\lambda_{0}\right) x_{n}\right\| \leqslant \frac{C}{2 \pi} r^{k}\left\|x_{n}\right\| .
$$

Ясно, что из неравенства $r / \gamma<1$ вытекает сходимость $(r / \gamma)^{k} \rightarrow 0$ при $k \rightarrow \infty$. Это означает, что $P_{n}\left(\lambda_{0}\right) \stackrel{\mathcal{P P}}{\longrightarrow} P\left(\lambda_{0}\right)$ компактно.

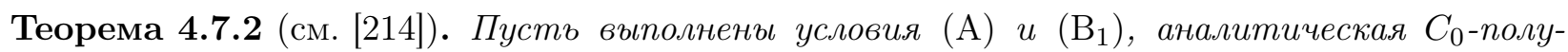
группа $e^{t A}, t \in \overline{\mathbb{R}}^{+}$, является гиперболической, причем множество $\sigma(A) \cap\{\lambda: \operatorname{Re} \lambda \geqslant 0\}$ состоит из конечного числа точек $P \sigma(A) u \operatorname{dim} P(\sigma+)<\infty$. Предположим также, что $\mu\left(\left\{B_{n} x_{n}\right\}\right) \leqslant q \mu\left(\left\{x_{n}\right\}\right)$, для любой ограниченной последовательности $\left\{x_{n}\right\}, x_{n} \in E_{n}$, где $q<1 u$ $B_{n}=e^{1 A_{n}}$. Тогда имеют место оценки (4.7.1) и сходимость $P_{n}\left(\sigma_{n}+\right) \stackrel{\mathcal{P P}}{\longrightarrow} P(\sigma+)$ компактна.

Доказательство. Согласно теореме о спектральном отображении спектр оператора $B=e^{1 A}$, расположенный вне единичного круга $\mathbb{T}$, состоит из конечного числа точек множества

$$
P \sigma\left(e^{1 A}\right)=\left\{\zeta: \zeta=e^{\lambda}, \lambda \in P \sigma(A) \cap\{\xi: \operatorname{Re} \xi \geqslant 0\}\right\} .
$$

Кроме того, поскольку $q<1$, для любого множества $\Psi \supset \mathbb{T}, \Psi \subset \rho(B), B=e^{1 A}$, имеем $\left\|\left(\lambda I_{n}-B_{n}\right)^{-1}\right\| \leqslant$ const при $\lambda \in \Psi$ согласно предложению 4.7.2. Из теоремы 4.6.5 следует, что $B_{n}$ имеют дискретную дихотомию. По теореме 4.6.2 оператор $\mathcal{D}_{n}$ обратим, а согласно предложению 4.6.1 и теореме 4.6.1 заключаем, что полугруппы $e^{t A_{n}}, t \in \mathbb{R}_{+}$, имеют экспоненциальную дихотомию равномерно по индексу $n \in \mathbb{N}$ и имеет место (4.7.1). Согласно предложению 4.7.2 имеем, что $P_{n}\left(\lambda_{0}\right) \stackrel{\mathcal{P} \mathcal{P}}{\longrightarrow} P\left(\lambda_{0}\right)$ компактно, откуда следует, что условия теоремы 4.7.1 выполнены. Теорема 4.7.2 доказана.

\section{8. ОБЩАЯ ДИХОТОМИЯ ПРИ ДИСКРЕТИЗАЦИИ ПО ВРЕМЕННО́Й ПЕРЕМЕННОЙ}

Рассмотрим дискретизацию задачи (4.2.11) по времени по следующей схеме:

$$
\frac{V_{n}\left(t+\tau_{n}\right)-V_{n}(t)}{\tau_{n}}=A_{u_{n}^{*}, n} V_{n}\left(t+\tau_{n}\right)+F_{u_{n}^{*}, n}\left(V_{n}(t)\right), \quad t=k \tau_{n},
$$

с начальными данными $V_{n}(0)=v_{n}^{0}$. Решение этой задачи дается формулой

$$
\begin{aligned}
V_{n}\left(t+\tau_{n}\right)= & \left(I_{n}-\tau_{n} A_{u_{n}^{*}, n}\right)^{-1} V_{n}(t)+\tau_{n}\left(I_{n}-\tau_{n} A_{u_{n}^{*}, n}\right)^{-1} F_{u_{n}^{*}, n}\left(V_{n}(t)\right)= \\
& =\left(I_{n}-\tau_{n} A_{u_{n}^{*}, n}\right)^{-k} V_{n}(0)+\tau_{n} \sum_{j=0}^{k}\left(I_{n}-\tau_{n} A_{u_{n}^{*}, n}\right)^{-(k-j+1)} F_{u_{n}^{*}, n}\left(V_{n}\left(j \tau_{n}\right)\right), \quad t=k \tau_{n},
\end{aligned}
$$

где $V_{n}(0)=v_{n}^{0}$.

Задачу (4.2.16) можно также дискретизировать по схеме (4.8.1), в результате чего получается задача (4.4.6). Решение задачи (4.4.6) выражается формулами

$$
\begin{gathered}
\left(I_{n}-P_{n}\right) V_{n}\left(t+\tau_{n}\right)=\left(I_{n}-\tau_{n} A_{u_{n}^{*}, n}\right)^{-1}\left(I_{n}-P_{n}\right) V_{n}(t)+\tau_{n}\left(I_{n}-\tau_{n} A_{u_{n}^{*}, n}\right)^{-1}\left(I_{n}-P_{n}\right) F_{u_{n}^{*}, n}\left(V_{n}\left(j \tau_{n}\right)\right), \\
\left(I_{n}-\tau_{n} A_{u_{n}^{*}, n}\right) P_{n} V_{n}\left(t+\tau_{n}\right)=P_{n} V_{n}(t)+\tau_{n} P_{n} F_{u_{n}^{*}, n}\left(V_{n}\left(k \tau_{n}\right)\right), \quad t=k \tau_{n} .
\end{gathered}
$$

Решение задачи (4.4.6) можно представить в виде (4.4.7). Из (4.4.7) ясно, что соответствующие оценки степеней операторов

$$
\left(I_{n}-\tau_{n} A_{u_{n}^{*}, n}\right)^{-k+1}\left(I_{n}-P_{n}\right), \quad\left(I_{n}-\tau_{n} A_{u_{n}^{*}, n}\right)^{K-k} P_{n}
$$

играют важную роль в аппроксимации решениий задачи (4.1.6) в окрестности точки $u^{*}$.

Теорема 4.8.1 (см. [86]). Пусть операторы $A_{n}, A$ являются генераторами аналитических $C_{0}$-полугрупп и выполнено условие $\left(\mathrm{B}_{1}\right)$. Предположим также, что аналитическал $C_{0}$-полугруппа $e^{t A_{u^{*}}}, t \in \overline{\mathbb{R}}^{+}$, является гиперболической и для числа $\rho>0$, удовлетворяющего условию $\{\lambda:-\rho \leqslant \operatorname{Re} \lambda \leqslant \rho\} \subset \rho(A)$, операторы $\lambda I_{n}-A_{u_{n}^{*}, n}$ являются фредгольмовыми операторами 
индекса 0, а операторы $\lambda I_{n}-A_{u_{n}^{*}, n}, \lambda I-A_{u^{*}}$ собственно согласованы для любого $\operatorname{Re} \lambda \geqslant-\rho$. Тогда $P_{n}\left(\sigma_{n}+\right) \stackrel{\mathcal{P P}}{\longrightarrow} P(\sigma+)$ компактно и

$$
\begin{cases}\left\|\left(I_{n}-\tau_{n} A_{u_{n}^{*}, n}\right)^{-k_{n}}\left(I_{n}-P_{n}\right)\right\|_{E_{n}} \leqslant M_{2} e^{-\gamma t}, & t \geqslant 0, \\ \left\|\left(I_{n}-\tau_{n} A_{u_{n}^{*}, n}\right)^{k_{n}} P_{n}\right\|_{E_{n}} \leqslant M_{2} e^{\gamma t}, & t \leqslant 0,\end{cases}
$$

для некоторого $\gamma>0$.

Доказательство. Из теоремы 4.7.1 следует, что $C_{0}$-полугруппы $e^{t A_{u_{n}^{*}, n}}$ имеют дихотомию равномерно по $n \in \mathbb{N}$. Как и в [24], видим, что

$$
\left\|\left(I_{n}-\tau_{n} A_{u_{n}^{*}, n}\right)^{-k_{n}}-e^{t A_{u_{n}^{*}, n}}\right\| \leqslant \frac{M}{t} \tau_{n} e^{\omega t},
$$

где $t=k_{n} \tau_{n}=1$. Используя теорему о возмущенной дихотомии (см. [136, с. 254]), заключаем, что выполнены неравенства (4.8.2). Теорема доказана.

\subsection{1. Дискретизация по временно́й переменной и свойство уплотнения.}

Теорема 4.8.2 (см. [86]). Предположим, что выполнены условия (A) $u\left(\mathrm{~B}_{1}\right) u \mu\left(\left\{B_{n} x_{n}\right\}\right) \leqslant q \mu\left(\left\{x_{n}\right\}\right)$ для любой ограниченной последовательности $\left\{x_{n}\right\}, x_{n} \in E_{n}$, где $q<1 u B_{n}=e^{A_{u_{n}^{*}, n}}$. Предположим такэе, что аналитическая $C_{0}$-полугруппа $e^{t A_{u^{*}}}, t \in \overline{\mathbb{R}}^{+}$, является гиперболической. Тогда

әде $r<1$.

$$
\begin{cases}\left\|\left(I_{n}-\tau_{n} A_{u_{n}^{*}, n}\right)^{-k_{n}}\left(I_{n}-P_{n}\right)\right\|_{E_{n}} \leqslant M_{2} r^{[t]}, & t=k_{n} \tau_{n} \geqslant 0, \\ \left\|\left(I_{n}-\tau_{n} A_{u_{n}^{*}, n}\right)^{k_{n}} P_{n}\right\|_{E_{n}} \leqslant M_{2} r^{-[t]}, & t=-k_{n} \tau_{n} \leqslant 0,\end{cases}
$$

Доказательство. Из теоремы 4.7.2 следует, что выполнены неравенства (4.2.15). Аналогично доказательству теоремы 4.8.1 получаем (4.8.3) по теореме о возмущении из [136]. Теорема 4.8.2 доказана.

Замечание 4.8.1. Имеем $\left\|F_{u_{n}^{*}, n}^{\prime}\left(w_{n}\right)\right\| \leqslant c_{\rho}\left\|w_{n}\right\|_{E_{n}^{\theta}}$, где $c_{\rho} \rightarrow 0$ при $\rho \rightarrow 0$. Здесь $\rho>0$ - радиус шаров $\mathcal{U}_{E_{n}^{\theta}}(0 ; \rho)$, для которых существует такое $\delta>0$, что

$$
\sup _{n \in \mathbb{N}} \sup _{\left\|w_{n}\right\|_{E_{n}^{\theta} \leqslant \delta}}\left\|f_{n}^{\prime}\left(w_{n}+p_{n}^{\theta} u^{*}\right)-f_{n}^{\prime}\left(p_{n}^{\theta} u^{*}\right)\right\|_{B\left(E_{n}^{\theta}, E_{n}\right)} \leqslant \rho .
$$

\section{9. ПОЛУДИСКРЕТНАЯ ДИХОТОМИЯ ДЛЯ УПЛОТНЯющИХ ОПЕРАТОРОв}

Предложение 4.9.1 (см. [214]). Предположим, что условия предложения 4.7.2 выполнены для $B=e^{A_{u^{*}}} u B_{n}=e^{A_{u_{n}^{*}, n}}$, где $q<1$, и полугруппа $e^{t A_{u^{*}}}, t \in \overline{\mathbb{R}}^{+}$, является гиперболической. Тогда справедлива теорема 4.7.1, в частности, имеет место (4.7.1).

4.9.1. Затенение при дискретизации по пространству и свойство уплотнения. Если резольвента оператора $A$ не является компактной, то мы получаем свойство затенения, но только на компактных подмножествах начальных данных $\mathcal{K} \subset \mathcal{U}_{E^{\theta}}\left(u^{*} ; \rho\right)$, как указано в следующей теореме.

Теорема 4.9.1 (см. [86]). Пусть оператор А является генератором экспоненииально убывающей аналитической $C_{0}$-полугруппы и $u^{*}$ - гиперболическая точка равновесия задачи (4.1.5). Предположсим, что $\left\{\lambda:-\rho_{1} \leqslant \operatorname{Re} \lambda \leqslant \rho_{1}\right\} \subset \rho(A)$, операторы $\lambda I_{n}-A_{u_{n}^{*}, n}$ и $\lambda I-A_{u^{*}}$ собственно согласованы для любого $\operatorname{Re} \lambda \geqslant-\rho_{1}, f_{n}\left(x_{n}\right) \stackrel{\mathcal{P}}{\longrightarrow} f(x)$ u $f_{n}^{\prime}\left(x_{n}\right) \stackrel{\mathcal{P}^{\theta} \mathcal{P}}{\longrightarrow} f^{\prime}(x)$ nри $x_{n} \stackrel{\mathcal{P}^{\theta}}{\longrightarrow} x u$ выполнены условия $\left(\mathrm{B}_{1}\right)$ и (F1)-(F3). Тогда существует $\rho_{0}>0$, обладающее следующим свойством: для любого компактного множества $\mathcal{K} \subset \mathcal{U}_{E^{\theta}}\left(u^{*} ; \rho\right)$ и любого $\epsilon_{0}>0$ найдется такое $n\left(\epsilon_{0}\right) \in \mathbb{N}$, что для произвольного обобщенного решения $u(t)$ задачи (4.1.5), удовлетворящего 
условию $u(\cdot) \in \mathcal{U}_{E^{\theta}}\left(u^{*} ; \rho_{0}\right), 0 \leqslant t \leqslant T$, для некоторого $0<T \leqslant \infty u u(0) \in \mathcal{K}$, существуют такие начальные значения $u_{n}^{0} \in E_{n}^{\theta}, n \geqslant n\left(\epsilon_{0}\right)$, что обобщенное решение $u_{n}\left(t ; u_{n}^{0}\right)$ задачи $(4.2 .9)$ существует на отрезке $[0, T]$ и удовлетворлет оченке

$$
\sup _{0 \leqslant t \leqslant T}\left\|u_{n}\left(t ; u_{n}^{0}\right)-\tilde{p}_{n}^{\theta} u(t)\right\|_{E_{n}^{\theta}} \leqslant \epsilon_{0} \quad \forall n \geqslant n\left(\epsilon_{0}\right)
$$

Доказательство. Используя вместо условия $\Delta_{c c} \neq \varnothing$ в теореме 4.4.1 условие $\left(\mathrm{B}_{1}\right)$ и собственную согласованность операторов $\lambda I_{n}-A_{n}$ и $\lambda I-A$ при $\operatorname{Re} \lambda \geqslant-\rho_{1}$, получим компактную сходимость $P_{n} \stackrel{\mathcal{P P}}{\longrightarrow} P$ и оценки (4.7.1). Ясно, что выполнены все утверждения теоремы 4.4.1. Поскольку множество $\mathcal{K} \subset \mathcal{U}_{E^{\theta}}(0 ; \rho)$ компактно, множество всех орбит

$$
\left\{u\left(t ; u^{0}\right): 0 \leqslant t \leqslant T \leqslant \infty, u^{0} \in \mathcal{K}\right\} \cup\left\{u^{*}\right\}
$$

компактно в $E$. Тогда доказательство вполне аналогично доказательству теоремы 4.4.1.

Теорема 4.9.2 (см. [86]). Пусть оператор А является генератором экспоненциально убывающей аналитической $C_{0}$-полугруппы и и* - гиперболическая точка равновесия задачи (4.1.5). Предположим, что $\left\{\lambda:-\rho_{1} \leqslant \operatorname{Re} \lambda \leqslant \rho_{1}\right\} \subset \rho(A)$, операторы $\lambda I_{n}-A_{u_{n}^{*}, n}$ u $\lambda I-A_{u^{*}}$ собственно согласованы для любого $\operatorname{Re} \lambda \geqslant-\rho_{1}, f_{n}\left(x_{n}\right) \stackrel{\mathcal{P}}{\longrightarrow} f(x)$ u $f_{n}^{\prime}\left(x_{n}\right) \stackrel{\mathcal{P}^{\theta} \mathcal{P}}{\longrightarrow} f^{\prime}(x)$ nри $x_{n} \stackrel{\mathcal{P}^{\theta}}{\longrightarrow} x u$ выполнены условия $\left(\mathrm{B}_{1}\right)$ и $(\mathrm{F} 1)-(\mathrm{F} 3)$. Тогда существует $\rho_{0}>0$, обладающее следующим свойством: для любого компактного множества $\mathcal{K} \subset \mathcal{U}_{E^{\theta}}\left(u^{*} ; \rho_{0}\right)$ и любого $\epsilon_{0}>0$ найдется такое $n\left(\epsilon_{0}\right) \in \mathbb{N}$, что для произвольной последовательности $\left\{u_{n}(\cdot)\right\}$ обобщенных решений задачи (4.2.9) с последовательностью $\left\{u_{n}^{0}\right\}, u_{n}(0)=u_{n}^{0} \in \mathcal{K}_{n}=p_{n}^{\theta} \mathcal{K} \cap \mathcal{U}_{E_{n}^{\theta}}\left(u_{n}^{*} ; \rho_{0}\right)$, существуют такие начальные значения $u^{n, 0} \in \mathcal{K}, n \geqslant n\left(\epsilon_{0}\right)$, что обобщенные решения $u\left(t ; u^{n, 0}\right)$ задачи (4.1.5) существуют на отрезке $[0, T]$ и удовлетворяют оченке

$$
\sup _{0 \leqslant t \leqslant T}\left\|u_{n}(t)-\tilde{p}_{n}^{\theta} u\left(t ; u^{n, 0}\right)\right\|_{E_{n}^{\theta}} \leqslant \epsilon_{0} \quad \forall n \geqslant n\left(\epsilon_{0}\right) .
$$

Доказательство. Согласно [79, Lemma 4.5] найдется такая последовательность $\left\{u^{n, 0}\right\}, u^{n, 0} \in \mathcal{K}$, что

$$
\left\|u_{n}^{0}-\tilde{p}_{n}^{\theta} u^{n, 0}\right\| \leqslant \epsilon_{0} \quad \text { при } n \geqslant n\left(\epsilon_{0}\right) .
$$

Следуя схеме доказательства теоремы 4.4.2, можно показать, что теорема 4.4.1 верна и, в частности, имеет место (4.9.2).

Теорема 4.9 .3 (см. [86]). Пусть оператор А является генератором экспоненииально убывающей аналитической $C_{0}$-полугруппы и $u^{*}$ - гиперболическая точка равновесия задачи (4.1.5). Предположсим, что $\mu\left(\left\{B_{n} x_{n}\right\}\right) \leqslant q \mu\left(\left\{x_{n}\right\}\right)$ для любой ограниченной последовательности $\left\{x_{n}\right\}$, $x_{n} \in E_{n}, q<1$, где $B_{n}=e^{1 A_{n}}, f_{n}\left(x_{n}\right) \stackrel{\mathcal{P}}{\longrightarrow} f(x)$ u $f_{n}^{\prime}\left(x_{n}\right) \stackrel{\mathcal{P}^{\theta} \mathcal{P}}{\longrightarrow} f^{\prime}(x)$ nрu $x_{n} \stackrel{\mathcal{P}^{\theta}}{\longrightarrow} x u$ выполнены условия (B) и (F1)-(F3). Тогда существует $\rho_{0}>0$, обладающее следующим свойством: для любого компактного множсества $\mathcal{K} \subset \mathcal{U}_{E^{\theta}}\left(u^{*} ; \rho_{0}\right)$ и любого $\epsilon_{0}>0$ найдется такое $n\left(\epsilon_{0}\right) \in \mathbb{N}$, что для произвольного обобщенного решения $и(t)$ задачи (4.1.5), удовлетворяющего условию $u(\cdot) \in \mathcal{U}_{E^{\theta}}\left(u^{*} ; \rho_{0}\right), 0 \leqslant t \leqslant T$, при некотором $0<T \leqslant \infty u u(0) \in \mathcal{K}$ существуют такие начальные значения $u_{n}^{0} \in E_{n}^{\theta}, n \geqslant n\left(\epsilon_{0}\right)$, что обобщенное решение $u_{n}\left(t ; u_{n}^{0}\right)$ задачи $(4.2 .9)$ существует на отрезке $[0, T]$ и удовлетворяет оценке (4.9.1).

Доказательство. Согласно теореме 4.7 .2 , из условия $\mu\left(\left\{B_{n} x_{n}\right\}\right) \leqslant q \mu\left(\left\{x_{n}\right\}\right)$ следует компактная сходимость $P_{n} \stackrel{\mathcal{P P}}{\longrightarrow} P$ и оценка (4.7.1); далее следуем схеме доказательства теоремы 4.9.1.

Теорема 4.9.4 (см. [86]). Пусть оператор А является генератором экспоненциально убывающей аналитической $C_{0}$-полугруппы и и* - гиперболическая точка равновесия задачи (4.1.5). Предположим, что $\mu\left(\left\{B_{n} x_{n}\right\}\right) \leqslant q \mu\left(\left\{x_{n}\right\}\right)$ для любой ограниченной последовательности $\left\{x_{n}\right\}$, $x_{n} \in E_{n}, q<1$, где $B_{n}=e^{1 A_{n}}, f_{n}\left(x_{n}\right) \stackrel{\mathcal{P}}{\longrightarrow} f(x)$ u $f_{n}^{\prime}\left(x_{n}\right) \stackrel{\mathcal{P}^{\theta} \mathcal{P}}{\longrightarrow} f^{\prime}(x)$ при $x_{n} \stackrel{\mathcal{P}^{\theta}}{\longrightarrow} x$ и выполненъ условия $\left(\mathrm{B}_{1}\right)$ и (F1)-(F3). Тогда существует $\rho_{0}>0$, обладающее следующим свойством: для 
любого компактного множества $\mathcal{K} \subset \mathcal{U}_{E^{\theta}}\left(u^{*} ; \rho_{0}\right)$ и любого $\epsilon_{0}>0$ найдется такое $n_{0}\left(\epsilon_{0}\right) \in \mathbb{N}$, что для произвольной последовательности $\left\{u_{n}(\cdot)\right\}$ обобщенных решений задач (4.2.9) с последовательностями $\left\{u_{n}^{0}\right\}, u_{n}(0)=u_{n}^{0} \in \mathcal{K}_{n}=p_{n}^{\theta} \mathcal{K} \cap \mathcal{U}_{E_{n}^{\theta}}\left(u_{n}^{*} ; \rho_{0}\right)$ существуют такие началънье значения $u^{n, 0} \in \mathcal{K}, n \geqslant n_{0}$, что обобщенные решения $u\left(t ; u^{n, 0}\right)$ задач (4.1.5) существуют на отрезке $[0, T]$ и удовлетворяют оценке (4.9.2).

Доказательство. Отметим, что из равномерных по $n \in \mathbb{N}$ оценок $\mu\left(\left\{B_{n} x_{n}\right\}\right) \leqslant q \mu\left(\left\{x_{n}\right\}\right), q<1$, следует дихотомия, как в (4.7.1). Дальнейшие рассуждения проводятся, как в доказательстве теоремы 4.9.2.

4.9.2. Затенение при дискретизации по временно́й переменной и свойство уплотнения. Леммы 4.4.1-4.4.3 справедливы при выполнении условий теорем 4.8.1-4.8.2, поскольку имеет место дискретная дихотомия и компактная сходимость $P_{n} \stackrel{\mathcal{P P}}{\longrightarrow} P$.

Теорема 4.9 .5 (см. [86]). Пусть оператор А является генератором экспоненииально убывающей аналитической $C_{0}$-полугруппь и $u^{*}$ - гиперболическая точка равновесия задачи (4.1.5). Предположим, что $\left\{\lambda:-\rho_{1} \leqslant \operatorname{Re} \lambda \leqslant \rho_{1}\right\} \subset \rho(A)$, операторь $\lambda I_{n}-A_{u_{n}^{*}, n}$ u $\lambda I-A_{u^{*}}$ собственно согласованы для любого $\operatorname{Re} \lambda \geqslant-\rho_{1}, f_{n}\left(x_{n}\right) \stackrel{\mathcal{P}}{\longrightarrow} f(x)$ u $f_{n}^{\prime}\left(x_{n}\right) \stackrel{\mathcal{P}^{\theta} \mathcal{P}}{\longrightarrow} f^{\prime}(x)$ nрu $x_{n} \stackrel{\mathcal{P}^{\theta}}{\longrightarrow} x u$ выполнены условия $\left(\mathrm{B}_{1}\right)$ u $(\mathrm{F} 1)-(\mathrm{F} 3)$. Тогда существует $\rho_{0}>0$, обладающее следующим свойством: для любого компактного множества $\mathcal{K} \subset \mathcal{U}_{E^{\theta}}(0 ; \rho)$ и любого $\epsilon_{0}>0$ найдется такое $n\left(\epsilon_{0}\right) \in \mathbb{N}$, что для произвольного обобщенного решения $v(t)$ задачи (4.1.10), удовлетворяющего условию $v(t) \in \mathcal{U}_{E^{\theta}}\left(0 ; \rho_{0}\right), 0 \leqslant t \leqslant T$, при некотором $0<T \leqslant \infty$, и $v^{0}, v^{T} \in \mathcal{K} \cap \mathcal{U}_{E^{\theta}}(0 ; \rho)$ существуют такие элементы $v_{n}^{0}, v_{n}^{T} \in E_{n}^{\theta}, n \geqslant n\left(\epsilon_{0}\right)$, что решения $V_{n}(\cdot)$ задач (4.4.6) существуют и удовлетворяют оценке

$$
\sup _{0 \leqslant t \leqslant T}\left\|V_{n}\left(t ; v_{n}^{0}, v_{n}^{T}\right)-\tilde{p}_{n}^{\theta} v\left(t ; v^{0}, v^{T}\right)\right\|_{E_{n}^{\theta}} \leqslant \epsilon_{0} \quad \text { npu } n \geqslant n\left(\epsilon_{0}\right) .
$$

Доказательство. Как и в теореме 4.4.4, рассмотрим решения (4.2.17) и (4.4.7). Требуемые оценки вытекают из теоремы 4.8.1.

Теорема 4.9 .6 (см. [86]). Пусть оператор А является генератором экспоненииально убывающей аналитической $C_{0}$-полугруппь и $u^{*}$ - гиперболическая точка равновесия задачи (4.1.5). Предположим, что $\left\{\lambda:-\rho_{1} \leqslant \operatorname{Re} \lambda \leqslant \rho_{1}\right\} \subset \rho(A)$, операторь $\lambda I_{n}-A_{u_{n}^{*}, n} u \lambda I-A_{u^{*}}$ собственно согласованьь для любого $\operatorname{Re} \lambda \geqslant-\rho_{1}, f_{n}\left(x_{n}\right) \stackrel{\mathcal{P}}{\longrightarrow} f(x)$ u $f_{n}^{\prime}\left(x_{n}\right) \stackrel{\mathcal{P}^{\theta} \mathcal{P}}{\longrightarrow} f^{\prime}(x) n p u x_{n} \stackrel{\mathcal{P}^{\theta}}{\longrightarrow} x u$ выполнены условия $\left(\mathrm{B}_{1}\right)$ и $(\mathrm{F} 1)-(\mathrm{F} 3)$. Тогда существует $\rho_{0}>0$, обладающее следующим свойством: для любого компактного множества $\mathcal{K} \subset \mathcal{U}_{E^{\theta}}\left(0 ; \rho_{0}\right)$ и любого $\epsilon_{0}>0$ найдется такое $n\left(\epsilon_{0}\right) \in \mathbb{N}$, что для произвольной последовательности $\left\{V_{n}(\cdot)\right\}$ решений задач (4.4.6) с последовательностями $\left\{v_{n}^{0}\right\},\left\{v_{n}^{T}\right\}$ из пространств $E_{n}^{\theta} u v_{n}^{0}, v_{n}^{T} \in \mathcal{K}_{n}=p_{n}^{\theta} \mathcal{K} \cap \mathcal{U}_{E_{n}^{\theta}}(0 ; \rho)$ существует такое множество элементов $v^{n, 0}, v^{n, T} \in E^{\theta}$, что обобщенные решения $v\left(t ; v^{n, 0}, v^{n, T}\right)$ задач (4.1.10) существуют на отрезке $[0, T]$ и удовлетворяют оценке

$$
\sup _{0 \leqslant t \leqslant T}\left\|V_{n} b\left(t ; v_{n}^{0}, v_{n}^{T}\right)-\tilde{p}_{n}^{\theta} v\left(t ; v^{n, 0}, v^{n, T}\right)\right\|_{E_{n}^{\theta}} \leqslant \epsilon_{0} \quad \text { npu } n \geqslant n\left(\epsilon_{0}\right) .
$$

Доказательство основано на теоремах 4.4.5 и 4.8.1.

Теорема 4.9.7 (см. [86]). Пусть оператор А является генератором экспоненциалъно убъвающей аналитической $C_{0}$-полугруппь и $u^{*}$ - гиперболическая точка равновесия задачи (4.1.5). Предположим, что $\mu\left(\left\{B_{n} x_{n}\right\}\right) \leqslant q \mu\left(\left\{x_{n}\right\}\right)$ для любой ограниченной последователъности $\left\{x_{n}\right\}$, $x_{n} \in E_{n}, q<1$, где $B_{n}=e^{1 A_{n}}, f_{n}\left(x_{n}\right) \stackrel{\mathcal{P}}{\longrightarrow} f(x)$ u $f_{n}^{\prime}\left(x_{n}\right) \stackrel{\mathcal{P}^{\theta} \mathcal{P}}{\longrightarrow} f^{\prime}(x)$ при $x_{n} \stackrel{\mathcal{P}^{\theta}}{\longrightarrow} x$ и виюполненъ условия $\left(\mathrm{B}_{1}\right)$ и (F1)-(F3). Тогда существует $\rho_{0}>0$, обладающее следуюшими свойствами: для любого компактного множества $\mathcal{K} \subset \mathcal{U}_{E^{\theta}}(0 ; \rho)$ и любого $\epsilon_{0}>0$ найдется такое $n\left(\epsilon_{0}\right) \in \mathbb{N}$, что для произвольного обобщенного решения $v(t)$ задачи (4.1.10), удовлетворяющего условию 
$v(t) \in \mathcal{U}_{E^{\theta}}\left(0 ; \rho_{0}\right), 0 \leqslant t \leqslant T$, при некотором $0<T \leqslant \infty u v^{0}, v^{T} \in \mathcal{K} \cap \mathcal{U}_{E^{\theta}}(0 ; \rho)$, существуют такие элементы $v_{n}^{0}, v_{n}^{T} \in E_{n}^{\theta}, n \geqslant n\left(\epsilon_{0}\right)$, что решения $V_{n}(\cdot)$ задач (4.4.6) существуют $u$ удовлетворяют оценке (4.9.3).

Доказательство основано на теоремах 4.8.2 и 4.9.5.

Теорема 4.9 .8 (см. [86]). Пусть оператор А является генератором экспоненииально убывающей аналитической $C_{0}$-полугруппы и $u^{*}$ - гиперболическая точка равновесия задачи (4.1.5). Предположим, что $\mu\left(\left\{B_{n} x_{n}\right\}\right) \leqslant q \mu\left(\left\{x_{n}\right\}\right)$ для любой ограниченной последовательности $\left\{x_{n}\right\}$, $x_{n} \in E_{n}, q<1$, где $B_{n}=e^{1 A_{n}}, f_{n}\left(x_{n}\right) \stackrel{\mathcal{P}}{\longrightarrow} f(x)$ u $f_{n}^{\prime}\left(x_{n}\right) \stackrel{\mathcal{P}^{\theta} \mathcal{P}}{\longrightarrow} f^{\prime}(x)$ при $x_{n} \stackrel{\mathcal{P}^{\theta}}{\longrightarrow} x$ и выполненъ условия $\left(\mathrm{B}_{1}\right)$ и (F1)-(F3). Тогда существует $\rho_{0}>0$, обладающее следующим свойством: для любого компактного множества $\mathcal{K} \subset \mathcal{U}_{E^{\theta}}\left(0 ; \rho_{0}\right)$ и любого $\epsilon_{0}>0$ найдется такое $n\left(\epsilon_{0}\right) \in \mathbb{N}$, что для произвольной последовательности $\left\{V_{n}(\cdot)\right\}$ решений задач (4.4.6) с последовательностями $\left\{v_{n}^{0}\right\},\left\{v_{n}^{T}\right\}$ из пространств $E_{n}^{\theta}$ u $v_{n}^{0}, v_{n}^{T} \in \mathcal{K}_{n}=p_{n}^{\theta} \mathcal{K} \cap \mathcal{U}_{E_{n}^{\theta}}(0 ; \rho)$ существует такое множество элементов $v^{n, 0}, v^{n, T} \in E^{\theta}$, что обобщенные решения $v\left(t ; v^{n, 0}, v^{n, T}\right)$ задач (4.1.10) существуют на отрезке $[0, T]$ и удовлетворяют оценке (4.9.4).

Доказательство основано на теоремах 4.8.2 и 4.9.6.

Замечание 4.9.1. В действительности не требуется производить выбор элементов $v^{T}$ и $v_{n}^{T}$ в зависимости от компактного множества $\mathcal{K}$, поскольку подпространства $P E$ и $P_{n} E_{n}$ конечномерны и операторы $p_{n}$ равномерно обратимы на $P_{n} E_{n}$ при $n \geqslant n_{0}$ для некоторого $n_{0}$.

\subsection{0. ПРИлОЖЕНИЯ С УПЛОТняющими УСЛОВИями}

Условие $\mu\left(B_{n} x_{n}\right) \leqslant q \mu\left(x_{n}\right), q<1$, в теоремах 4.7.2 и 4.8.2 можно проверить, например, в случае компактной сходимости операторов $A_{n}^{-1} f_{n}^{\prime}\left(u_{n}^{*}\right) \stackrel{\mathcal{P} \mathcal{P}}{\longrightarrow} A^{-1} f^{\prime}\left(u^{*}\right)$. Приведем пример, в котором аналогичное условие естественным образом выполнено.

Пример 4.10.1. Рассмотрим в пространстве $L^{2}(\mathbb{R})$ оператор

$$
(A v)(x)=v^{\prime \prime}(x)+a v^{\prime}(x)+b v(x), \quad x \in(-\infty, \infty) .
$$

Поскольку $E=L^{2}(\mathbb{R})$, можно положить

$$
\left(p_{n} v\right)(x)=\frac{1}{h} \int_{-h / 2}^{h / 2} v(x+y) d y,
$$

и основное условие $\left\|p_{n} v\right\|_{L_{h}^{2}(Z)} \rightarrow\|v\|_{L^{2}(R)}$ выполнено (см. [259]).

Как и в [136, Sec. 5.4], видим, что

$$
\sigma_{\text {ess }}(-A) \subset\left\{\lambda: \operatorname{Re} \lambda-\frac{(\operatorname{Im} \lambda)^{2}}{a^{2}} \geqslant-b\right\} .
$$

В случае $a=0$ имеем $\sigma(A) \in(-\infty, b)$. Таким образом, при $b<0$ оператор $A$ является отрицательным самосопряженным оператором. То же справедливо для некоторой разностной схемы, например, для центрально-разностной схемы

$$
A_{n} v_{n}(x)=\frac{v_{n}(x+h)-2 v_{n}(x)+v_{n}(x-h)}{h^{2}}+b v_{n}(x),
$$

т.е. $\omega_{\mathrm{ess}}\left(A_{n}\right) \leqslant \omega_{1}<0$ равномерно по $h>0$. Кроме того, легко видеть, что

$$
\left\|e^{t A_{n}}\right\| \leqslant M e^{\omega_{2} t}, \quad t \geqslant 0, \quad \omega_{2}<0
$$

т.e.

$$
\mu\left(\left\{e^{t A_{n}} x_{n}\right\}\right) \leqslant \gamma \mu\left(\left\{x_{n}\right\}\right), \quad \gamma<1, \quad \text { для некоторого } t=t_{0}>0 .
$$

Введем обозначения $B=e^{t_{0} A}$ и $B_{n}=e^{t_{0} A_{n}}$. Для аналитических $C_{0}$-полугрупп спектры операторов $A$ и $B$ строго связаны; то же касается и точечных спектров $P \sigma(B)=e^{t_{0} P \sigma(A)}$. Это означает, что 
для почти всех $n$ операторы $A_{n}$ имеют спектры $\sigma\left(A_{n}\right) \cap\{\lambda: \operatorname{Re} \lambda>0\}$, которые аппроксимируют спектр $\sigma(A) \cap\{\lambda: \operatorname{Re} \lambda>0\}$.

Рассмотрим в пространстве $L^{2}(\mathbb{R})$ возмущенный оператор с гладкой функцией $b(x)$,

$$
\tilde{A} v(x)=v^{\prime \prime}(x)+b(x) v(x),
$$

и его аппроксимацию, например,

$$
\left(\tilde{A}_{n} v_{n}\right)(x)=\frac{v_{n}(x+h)-2 v_{n}(x)+v_{n}(x-h)}{h^{2}}+b(x) v_{n}(x) ;
$$

для простоты наложим условие $b(x) \rightarrow b$ при $x \rightarrow \pm \infty$. Оператор

$$
((\tilde{A}-A) v)(x)=(b(x)-b) v(x)
$$

представляет собой аддитивное возмущение. Предположим, что $C_{0}$-полугруппа $e^{t \tilde{A}}, t \in \mathbb{R}_{+}$, является гиперболической. Возмущение $\tilde{A}-A$ является относительно компактным, как в [16]. Операторы $A_{n}$ обладают теми же свойствами, поскольку $\tilde{A}_{n}=A_{n}+\left(\tilde{A}_{n}-A_{n}\right)$ и

$$
e^{t_{0} \tilde{A}_{n}}=e^{t_{0} A_{n}}+\int_{0}^{t_{0}} A_{n}^{\theta} e^{\left(t_{0}-s\right) A_{n}} A_{n}^{-\theta}\left(\tilde{A}_{n}-A_{n}\right) e^{s \tilde{A}_{n}} d s .
$$

Важно отметить, что благодаря (4.10.1) такие возмущения дают оценку

$$
\mu\left(\left\{e^{t \tilde{A}_{n}} x_{n}\right\}\right) \leqslant \gamma \mu\left(\left\{x_{n}\right\}\right), \quad \text { где } \gamma<1, \text { при некотором } t=t_{0}>0,
$$

поскольку интегральное слагаемое в (4.10.2) можно оценить произвольным малым $\epsilon>0$ следующим образом:

$$
\mu\left(\int_{0}^{t_{0}-\epsilon}+\int_{t_{0}-\epsilon}^{t_{0}}\right) \leqslant c \epsilon^{1-\theta} .
$$

Тогда любая точка спектра оператора $\tilde{A}$, расположенная правее $b$, принадлежит $P \sigma(\tilde{A})$, и ей соответствует конечномерное корневое подпространство. То же верно для $\tilde{A}_{n}$, где $B_{n}=e^{t \tilde{A}_{n}}$, поскольку выполнено (4.10.3). Используя свойство (4.10.3), получим из теоремы 4.7.1 и предложения 4.7.2 собственную согласованность операторов $\lambda I_{n}-\tilde{A}_{n}$ и $\lambda I-\tilde{A}$ для любых $\lambda \in i \mathbb{R}$ и $\operatorname{Re} \lambda>b$.

Если, как и выше, $P$ - дихотомический проектор, то имеем $\operatorname{dim} P<\infty$ и $P \tilde{A}=\tilde{A} P$. Можно также доказать, что $P_{n} \rightarrow P$ компактно в силу предложения 4.7.2. Это означает, что, например, из дихотомии оператора $\tilde{A}$ получаем дихотомию для $\tilde{A}_{n}$ равномерно по $n$ согласно теореме 4.7.1. Аналогичная ситуация для конкретного дифференциального оператора была рассмотрена в [233].

\section{ГЛАВА 5}

\section{ПОЛУЛИНЕЙНЫЕ ДРОБНЫЕ УРАВНЕНИЯ}

В последние несколько лет дробные дифференциальные уравнения привлекли внимание многих исследователей благодаря многочисленным приложениям во многих областях науки: физике, математической биологии, химии, нелинейной динамике и т. д. (см., например, $[28,62,63,103,193$, $194,202,204])$. Существует мнение, что модели дробного порядка более реалистичны, чем классические модели (см. $[64,65,130,155,198,209,210])$. В этой главе обсуждаются полулинейные дробные уравнения в банаховых пространствах, интенсивное изучение которых только начинается (см. $[2,225,254])$. Мы рассматриваем только корректно поставленные задачи. Некорректные задачи для уравнений с производными целого порядка рассматривались, например, в $[14,177,179]$. Некорректные задачи для дробных уравнений будут рассмотрены в отдельной работе. 


\section{1. ПОСТАНОВКА ЗАДАЧИ}

Рассмотрим аппроксимацию задачи Коши

$$
\left(\mathcal{D}_{t}^{\alpha} u\right)(t)=A u(t)+J^{1-\alpha} f(t, u(t)), \quad 0 \leqslant t \leqslant T ; \quad u(0)=u^{0},
$$

где $\mathcal{D}_{t}^{\alpha}$ - производная Капуто-Джрбашяна, оператор $A$ порождает аналитическое и компактное разрешающее семейство $S_{\alpha}(\cdot, A)$, а функция $f(\cdot, \cdot)$ является достаточно гладкой.

Напомним некоторые определения (см. [155]). Дробный интеграл порядка $\alpha>0$ определяется формулой

$$
\left(J^{\alpha} q\right)(t):=\left(g_{\alpha} * q\right)(t), \quad t>0,
$$

где

$$
g_{\alpha}(t):= \begin{cases}\frac{t^{\alpha-1}}{\Gamma(\alpha)}, & t>0 \\ 0, & t \leqslant 0\end{cases}
$$

$\Gamma(\alpha)$ - гамма-функция. Производная Римана-Лиувилля порядка $\alpha>0$ определяется формулой

$$
\left(D_{t}^{\alpha} q\right)(t)=\left(\frac{d}{d t}\right)^{m}\left(J^{m-\alpha} q\right)(t)
$$

где $m=\lceil\alpha\rceil$, а дробная производная Капуто-Джрбашяна порядка $\alpha>0$-формулой

$$
\left(\mathcal{D}_{t}^{\alpha} q\right)(t)=\left(D_{t}^{\alpha} q\right)(t)-\sum_{k=0}^{m-1} \frac{q^{(k)}(0)}{\Gamma(k-\alpha+1)} t^{k-\alpha} .
$$

Определение 5.1.1. Семейство $\left\{S_{\alpha}(t, A)\right\}_{t \geqslant 0} \subset B(E)$ называется $\alpha$-разрешающим семейством, порожденным оператором $A$, если выполнены следующие условия:

(a) $S_{\alpha}(t, A)$ сильно непрерывно при $t \geqslant 0$ и $S_{\alpha}(0, A)=I$;

(b) $S_{\alpha}(t, A) D(A) \subseteq D(A)$ и $A S_{\alpha}(t, A) x=S_{\alpha}(t, A) A x$ для всех $x \in D(A), t \geqslant 0$;

(c) $S_{\alpha}(t, A) x$ удовлетворяет при $x \in D(A)$ разрешающему уравнению

$$
S_{\alpha}(t, A) x=x+\int_{0}^{t} g_{\alpha}(t-s) S_{\alpha}(s, A) A x d s, \quad t \geqslant 0 .
$$

Определение 5.1.2. $\alpha$-Разрешающее семейство $S_{\alpha}(\cdot, A)$ называется аналитическим, если $S_{\alpha}(\cdot, A)$ допускает аналитическое продолжение в сектор $\Sigma_{\theta_{0}} \backslash\{0\}$ для некоторого $\theta_{0} \in(0, \pi / 2]$, где $\Sigma_{\theta_{0}}:=\left\{\lambda \in \mathbb{C}:|\arg \lambda|<\theta_{0}\right\}$. Будем говорить, что аналитический $\alpha$-разрешающий оператор $S_{\alpha}(\cdot, A)$ имеет тип аналитичности $\left(\theta_{0}, \omega_{0}\right)$, если для всех $\theta<\theta_{0}$ и $\omega>\omega_{0}$ найдется такое $M=M(\theta, \omega)$ что

$$
\left\|S_{\alpha}(z, A)\right\| \leqslant M e^{\omega \operatorname{Re} z}, \quad z \in \Sigma_{\theta} .
$$

Определение 5.1.3. $\alpha$-Разрешающее семейство $S_{\alpha}(\cdot, A)$ называется компактным, если для любого $t>0$ оператор $S_{\alpha}(t, A)$ компактен.

Замечание 5.1.1. Аналитические и компактные $\alpha$-разрешающие семейства $S_{\alpha}(\cdot, A)$ существуют. Действительно, известно (см. [Chap. II, Sec. 4, 4.34. Exercises (1)] [106]), что оператор $A=d^{2} / d x^{2}, D(A)=\left\{f \in C^{2}[0,1]: f^{\prime}(0)=f^{\prime}(1)=0\right\}$, порождает компактную аналитическую сжимающую $C_{0}$-полугруппу в пространстве $E=C[0,1]$. Таким образом, из [1] и [66, теорема 3.3], вытекает, что $A$ порождает компактное аналитическое $\alpha$-разрешающее семейство $S_{\alpha}(\cdot, A)$ для любого $0<\alpha<1$.

В [66] доказано, что однородная задача Коши (5.1.1) корректно поставлена тогда и только тогда, когда $A$ порождает $\alpha$-разрешающее семейство $S_{\alpha}(\cdot, A)$. С самого начала предположим, что разрешающее семейство $S_{\alpha}(\cdot, A)$ удовлетворяет оценке

$$
\left\|S_{\alpha}(t, A)\right\| \leqslant M e^{\omega t}, \quad t \geqslant 0
$$


для некоторых $M, \omega>0$. В таком случае для $\left\{\lambda^{\alpha}: \operatorname{Re} \lambda>\omega\right\} \subset \rho(A)$ имеем

$$
\lambda^{\alpha-1}\left(\lambda^{\alpha} I-A\right)^{-1} x=\int_{0}^{\infty} e^{-\lambda t} S_{\alpha}(t, A) x d t, \quad \operatorname{Re} \lambda>\omega, \quad x \in E .
$$

Отметим, что в этой главе нас интересуют только корректно поставленные задачи Коши для дифференциальных уравнений порядка $0<\alpha<1$. Для ограниченного генератора $A$ семейство $S_{\alpha}(t, A)$ задается функцией Миттаг-Леффлера $E_{\alpha}\left(t^{\alpha} A\right)$ :

$$
S_{\alpha}(t, A)=E_{\alpha}\left(t^{\alpha} A\right)=\sum_{j=0}^{\infty} \frac{\left(t^{\alpha} A\right)^{j}}{\Gamma(\alpha j+1)} .
$$

Определение 5.1.4. Функция $u(\cdot) \in C([0, T] ; E)$ называется обобщенным решением задачи (5.1.1), если функция $u(\cdot)$ удовлетворяет уравнению

$$
u(t)=S_{\alpha}(t, A) u^{0}+\int_{0}^{t} S_{\alpha}(t-s, A) f(s, u(s)) d s .
$$

В [152] был предложен другой подход к обобщенным решениям. В этом разделе мы следуем определению 5.1.4.

Теорема 5.1.1 (см. $[109,175])$. Пусть $A$-генератор $\alpha$-разрешающего семейства $S_{\alpha}(\cdot, A)$. Предположим, что функиия $f(\cdot, \cdot):[0, T] \times E \rightarrow E$ непрерывна по $t \in[0, T]$ и существует такая константа $L>0$, что

$$
\|f(t, x)-f(t, y)\| \leqslant L\|x-y\| \quad \text { npu } t \in[0, T], x, y \in E .
$$

Тогда существует единственное обобщенное решение $u(\cdot) \in C([0, T] ; E)$ задачи (5.1.1).

Полудискретная аппроксимация задачи (5.1.1) на общей дискретизационной схеме - это множество задач Коши в банаховых пространствах $E_{n}$ вида

$$
\left(\mathcal{D}_{t}^{\alpha} u_{n}\right)(t)=A_{n} u_{n}(t)+J^{1-\alpha} f_{n}\left(t, u_{n}(t)\right), \quad 0 \leqslant t \leqslant T, \quad u_{n}(0)=u_{n}^{0},
$$

где операторы $A_{n}$ порождают аналитические и компактные $\alpha$-разрешающие семейства $S_{\alpha}\left(\cdot, A_{n}\right)$, а функции $f_{n}(\cdot, \cdot)$ являются достаточно гладкими. В следующем разделе будет установлена сходимость решений задач (5.1.5) к решению задачи (5.1.1).

Заметим, что аппроксимация линейной дробной задачи

$$
\left(\mathcal{D}_{t}^{\alpha} u\right)(t)=A u(t)+f(t), \quad u(0)=u^{0},
$$

изучалась в основном для случая гильбертовых пространств многими исследователями (см., например, $[36,149,182])$. Полудискретные аппроксимации дробных уравнений рассматривались в $[113,141,142]$.

\section{2. ПОЛУДИСКРЕТНАЯ АППРОКСИМАЦИЯ В СПЕЦИАЛЬНОМ СЛУЧАЕ}

Для аналитического $\alpha$-разрешающего семейства имеется особый вариант теоремы АВС (см. [186]). Для его доказательства нам потребуется следующая лемма.

Лемма 5.2.1 (см. [186]). Пусть функиии $f_{n}(\cdot) \in C\left(\mathbb{R}_{+} ; E_{n}\right)$ таковы, что $\left\|f_{n}(t)\right\|_{E_{n}} \leqslant M e^{\omega t}$ для некоторого $M>0, \omega \in \mathbb{R} u$ всех $n \in \mathbb{N}$ и пусть $\lambda_{0} \geqslant \omega$. Следующие условия эквивалентны:

(I) преобразования Лапласа $\hat{f}_{n}(\cdot)$ поточечно $Р$-сходятся на $\left(\lambda_{0}, \infty\right) \kappa \hat{f}(\cdot)$ и последовательность $\left\{f_{n}(\cdot)\right\}, n \in \mathbb{N}$, равномерно непрерывна на компактных подмножествах множества $\mathbb{R}_{+}$;

(II) функиии $f_{n}(\cdot)$ равномерно $Р$-сходятся на компактных подмножествах множества $\mathbb{R}_{+}$ $\kappa f(\cdot)$. 
Кроме того, если имеет место условие (II), то

$$
\hat{f}(\lambda)=\underset{n \rightarrow \infty}{\mathrm{P}-\lim } \hat{f}_{n}(\lambda)
$$

для всех $\lambda>\lambda_{0}$, где

$$
f(t):=\underset{n \rightarrow \infty}{\mathrm{P}-\lim } f_{n}(t)
$$

и $\hat{q}(\cdot)$ - преобразование Лапласа функиии $q(\cdot)$.

Теорема 5.2.1 (см. [188]). Предположим, что $0<\alpha \leqslant 2$ и операторы $A, A_{n}$ порождают экспоненциально ограниченные аналитические $\alpha$-разрешающие семейства $S_{\alpha}(\cdot, A), S_{\alpha}\left(\cdot, A_{n}\right)$ в

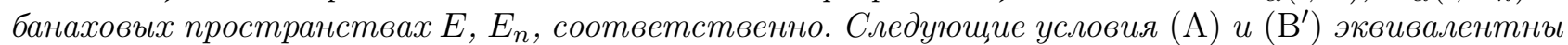
условию $\left(\mathrm{C}^{\prime}\right)$ :

(А) (согласованность): существует такое $\lambda \in \rho(A) \cap \bigcap_{n} \rho\left(A_{n}\right)$, что резольвенты сходятся:

$$
\left(\lambda I_{n}-A_{n}\right)^{-1} \stackrel{\mathcal{P P}}{\longrightarrow}(\lambda I-A)^{-1} ;
$$

$\left(\mathrm{B}^{\prime}\right)$ (устойчивость): найдутся такие константы $M \geqslant 1,0<\varphi \leqslant \pi / 2 u \omega$, не зависящие от $n$, что сектор $\omega+\Sigma_{\varphi+\pi / 2}$ содержится в $\rho\left(A_{n}\right) u$

$$
\sup _{\lambda \in \omega+\Sigma_{\beta+\pi / 2}}\left\|\lambda^{\alpha-1} R\left(\lambda^{\alpha} ; A_{n}\right)\right\|_{B\left(E_{n}\right)} \leqslant \frac{M}{|\lambda-\omega|}
$$

для любых $n \in \mathbb{N} u 0<\beta<\varphi$;

$\left(\mathrm{C}^{\prime}\right)$ (сходимость): для некоторого конечного $\omega_{1}>0$ имеем

$$
\sup _{z \in \Sigma_{\beta}} e^{-\omega_{1} \operatorname{Re} z}\left\|S_{\alpha}\left(z, A_{n}\right) x_{n}-p_{n} S_{\alpha}(z, A) x\right\|_{E_{n}} \rightarrow 0 \quad \text { npu } n \rightarrow \infty
$$

если $x_{n} \stackrel{\mathcal{P}}{\longrightarrow}$ хрии всех $x_{n} \in E_{n}, x \in E$ и любого $0<\beta<\varphi$.

Доказательство. Во-первых, предположим, что выполнены условия (А) и $\left(\mathrm{B}^{\prime}\right)$. Напомним (см. [66]), что условие $\left(\mathrm{B}^{\prime}\right)$ эквивалентно следующему условию: найдутся такие константы $C$, $\omega$ и $0<\varphi \leqslant \pi / 2$, что

$$
\left\|S_{\alpha}\left(z, A_{n}\right)\right\|_{B\left(E_{n}\right)} \leqslant C e^{\omega \operatorname{Re} z} \quad \text { для всех } z \in \Sigma(\beta), 0<\beta<\varphi \text {, равномерно по } n \in \mathbb{N} \text {. }
$$

Кроме того, можем записать (см. [66,227])

$$
S_{\alpha}\left(z, A_{n}\right)=\frac{1}{2 \pi i} \int_{\Gamma} e^{\lambda z} \lambda^{\alpha-1} R\left(\lambda^{\alpha} ; A_{n}\right) d \lambda,
$$

где $Г$ - положительно ориентированный контур, являющийся границей множества $\omega+\Sigma_{\beta+\pi / 2}$. Разделим этот контур на две части $\Gamma=\Gamma_{a} \cup \Gamma_{b}$, где

$$
\Gamma_{a}=\Gamma \cap\{z:|z| \leqslant a\}, \quad \Gamma_{b}=\Gamma \backslash \Gamma_{a} .
$$

Интеграл по $\Gamma_{b}$ можно сделать меньше произвольного $\varepsilon>0$ равномерно по $n$, если $a$ достаточно велико. Для любого $r>0$ найдется достаточно малое $\delta$, для которого при $z_{1}, z_{2} \in \Sigma(\beta) \cap\{z$ : $\operatorname{Re} z \leqslant r\} \backslash\{0\}$ и $\left|z_{1}-z_{2}\right|<\delta$ имеем

$$
\begin{aligned}
\left\|\int_{\Gamma_{a}} e^{\lambda z_{1}} \lambda^{\alpha-1} R\left(\lambda^{\alpha} ; A_{n}\right) d \lambda-\int_{\Gamma_{a}} e^{\lambda z_{2}} \lambda^{\alpha-1} R\left(\lambda^{\alpha} ; A_{n}\right) d \lambda\right\|_{B\left(E_{n}\right)} & = \\
& =\int_{\Gamma_{a}}\left|e^{\lambda z_{1}}-e^{\lambda z_{2}}\right|\left\|\lambda^{\alpha-1} R\left(\lambda^{\alpha} ; A_{n}\right)\right\|_{B\left(E_{n}\right)} d \lambda<\varepsilon .
\end{aligned}
$$

Итак, получили равностепенную непрерывность семейства $\left\{S_{\alpha}\left(z, A_{n}\right)\right\}$ на $\Sigma(\beta) \cap\{z: \operatorname{Re} z \leqslant r\} \backslash$ $\{0\}$. На самом деле мы доказали равностепенную непрерывность семейства $\left\{S_{\alpha}\left(z, A_{n}\right) x_{n}\right\}$ при 
$z=0$ (см. [186, теорема 7]). Поэтому $\left\{S_{\alpha}\left(z, A_{n}\right) x_{n}\right\}$ равностепенно непрерывно на $\Sigma(\beta) \cap\{z:$ $\operatorname{Re} z \leqslant r\}$. Тогда из леммы 5.2.1 следует, что

$$
\max _{z \in \Sigma_{\beta} \cap\{z: \operatorname{Re} z \leqslant r\}}\left\|S_{\alpha}\left(z, A_{n}\right) x_{n}-p_{n} S_{\alpha}(z, A) x\right\|_{E_{n}} \rightarrow 0 \quad \text { для всех } r>0 .
$$

С другой стороны, для любого $\varepsilon>0$ и выбранного $\omega_{1}>\omega$ существует такое $r_{0}>0$, что

$$
\max _{z \in \Sigma_{\beta} \cap\left\{z: \operatorname{Re} z>r_{0}\right\}} e^{-\omega_{1} \operatorname{Re} z}\left\|S_{\alpha}\left(z, A_{n}\right) x_{n}-p_{n} S_{\alpha}(z, A) x\right\|_{E_{n}}<\varepsilon, \quad n \geqslant n_{0} .
$$

Таким образом, получаем условие $\left(\mathrm{C}^{\prime}\right)$.

Если условие $\left(\mathrm{C}^{\prime}\right)$ выполнено, то условие (А) получаем из (5.1.4) при помощи теоремы Лебега о мажорантной сходимости. Из $\left(\mathrm{C}^{\prime}\right)$ следует и оценка

$$
\max _{z \in \Sigma(\beta)} e^{-\omega \operatorname{Re} z}\left\|S_{\alpha}\left(z, A_{n}\right)\right\|_{B\left(E_{n}\right)} \leqslant C
$$

что эквивалентно условию $\left(\mathrm{B}^{\prime}\right)$. Предположим, от противного, что это не так. Тогда найдутся такие последовательности $\left\|x_{n}\right\|_{E_{n}}=1$ и $z_{n} \in \Sigma(\beta)$, что

$$
e^{-\omega \operatorname{Re} z_{n}}\left\|S_{\alpha}\left(z_{n}, A_{n}\right) x_{n}\right\|_{E_{n}} \rightarrow \infty \text {. }
$$

В таком случае для последовательности

которая сходится к нулю, имеем

$$
y_{n}=\frac{x_{n}}{e^{-\omega \operatorname{Re} z_{n}}\left\|S_{\alpha}\left(z_{n}, A_{n}\right) x_{n}\right\|_{E_{n}}}
$$

$$
e^{-\omega \operatorname{Re} z_{n}} S_{\alpha}\left(z_{n}, A_{n}\right) y_{n} \rightarrow e^{-\omega \operatorname{Re} z_{n}} S_{\alpha}\left(z_{n}, A_{n}\right) 0=0 \quad \text { равномерно по } z_{n} \in \Sigma(\beta) .
$$

Это противоречит равенству $e^{-\omega \operatorname{Re} z_{n}}\left\|S_{\alpha}\left(z_{n}, A_{n}\right) y_{n}\right\|_{E_{n}}=1$.

Замечание 5.2.1. Из доказательства теоремы 5.2.1 получаем равностепенную непрерывность семейства $S_{\alpha}\left(\cdot, A_{n}\right)$ на любом компактном подмножестве множества $\overline{\mathbb{R}}^{+}$, если выполнены условия $(\mathrm{A})$ и $\left(\mathrm{B}^{\prime}\right)$.

Замечание 5.2.2. Можно получить равностепенную непрерывность семейства $\left\{R\left(\lambda^{\alpha} ; A_{n}\right)\right\}$ также на любом компактном подмножестве множества $\omega+\Sigma_{\beta+\pi / 2}$ из условия $\left(\mathrm{B}^{\prime}\right)$ : она вытекает из тождества Гильберта

$$
\left(\lambda^{\alpha} I_{n}-A_{n}\right)^{-1}-\left(\mu^{\alpha} I_{n}-A_{n}\right)^{-1}=\left(\mu^{\alpha}-\lambda^{\alpha}\right)\left(\lambda^{\alpha} I_{n}-A_{n}\right)^{-1}\left(\mu^{\alpha} I_{n}-A_{n}\right)^{-1}
$$

и неравенства (5.2.1).

Теорема 5.2.2 (см. [187]). Пусть оператор А является генератором экспоненииально ограниченного аналитического $\alpha$-разрешающего семейства $S_{\alpha}(\cdot, A)$ в банаховом пространстве $E$. Тогда компактность семейства $S_{\alpha}(t, A)$ для любого $t>0$ эквивалентна компактности резольвенты $R\left(\lambda^{\alpha} ; A\right)$, для любого $\lambda^{\alpha} \in \rho(A)$.

Доказательство. Предположим, что функция $S_{\alpha}(\cdot, A)$ аналитична и компактна. В силу представления

$$
R\left(\lambda^{\alpha} ; A\right)=\lambda^{1-\alpha} \int_{0}^{\infty} e^{-\lambda t} S_{\alpha}(t, A) d t
$$

заключаем, что оператор-функция

$$
R_{q, Q}(\lambda):=\lambda^{1-\alpha} \int_{q}^{Q} e^{-\lambda t} S_{\alpha}(t, A) d t
$$

аппроксимирует резольвенту:

$$
\left\|R\left(\lambda^{\alpha} ; A\right)-R_{q, Q}(\lambda)\right\| \rightarrow 0 \quad \text { при } q \rightarrow 0 \text { и } Q \rightarrow \infty .
$$


Оператор-функция $R_{q, Q}(\lambda)$ компактна (см. [264]). Таким образом, резольвента $R\left(\lambda^{\alpha} ; A\right)$ компактна как равномерный предел компактных операторов.

Обратно, используя (5.2.2), можем записать

$$
S_{\alpha}(t, A)=\frac{1}{2 \pi i} \int_{\Gamma} e^{\lambda t} \lambda^{\alpha-1} R\left(\lambda^{\alpha} ; A\right) d \lambda \quad \text { для всех } t>0 .
$$

Поскольку резольвента $R\left(\lambda^{\alpha} ; A\right)$ компактна для любого $\lambda^{\alpha} \in \rho(A)$, заключаем, как и выше, что $S_{\alpha}(t, A)$ компактно для любого $t \neq 0$.

Теорема 5.2.3 (см. [187]). Предположим, что $S_{\alpha}\left(\cdot, A_{n}\right)$ и $S_{\alpha}(\cdot, A)$ - аналитические $\alpha$-разрешающие семейства, порожденные операторами $A_{n} u A$ в банаховых пространствах $E_{n} u E$,

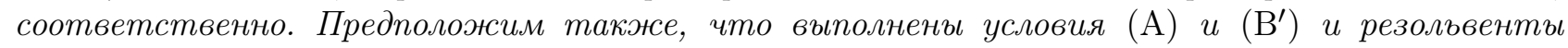
$R\left(\lambda^{\alpha} ; A_{n}\right)$ и $R\left(\lambda^{\alpha} ; A\right)$ компактны. Тогда следующие условия эквивалентны:

(1) $R\left(\lambda^{\alpha} ; A_{n}\right) \stackrel{\mathcal{P P}}{\longrightarrow} R\left(\lambda^{\alpha} ; A\right)$ компактно для некоторого $\lambda^{\alpha} \in \rho(A) \cap \bigcap_{n} \rho\left(A_{n}\right)$;

(2) $S_{\alpha}\left(t, A_{n}\right) \stackrel{\mathcal{P P}}{\longrightarrow} S_{\alpha}(t, A)$ компактно для любого $t>0$.

Доказательство. (1) $\Longrightarrow(2)$. Из теоремы 5.2 .1 следует, что $S_{\alpha}\left(t, A_{n}\right) \stackrel{\mathcal{P P}}{\longrightarrow} S_{\alpha}(t, A)$. Требуется показать, что семейство $\left\{S_{\alpha}\left(t, A_{n}\right) x_{n}\right\}$ является $P$-компактным для любой последовательности $\left\{x_{n}\right\},\left\|x_{n}\right\|_{E_{n}}=O(1)$. Пусть $\mu(\cdot)$ - дискретная мера некомпактности последовательностей. Для любого $t>0$, используя (5.2.2), получим

$$
\begin{aligned}
& \left.\mu\left(\left\{S_{\alpha}\left(t, A_{n}\right) x_{n}\right\}\right)=\mu\left(\left\{\frac{1}{2 \pi i} \int_{\Gamma} e^{\lambda t} \lambda^{\alpha-1} R\left(\lambda^{\alpha} ; A_{n}\right) x_{n}\right], d \lambda\right\}\right)= \\
& =\mu\left(\left\{\frac{1}{2 \pi i} \int_{\Gamma_{a}} e^{\lambda t} \lambda^{\alpha-1} R\left(\lambda^{\alpha} ; A_{n}\right) x_{n} d \lambda\right\}\right)+\mu\left(\left\{\frac{1}{2 \pi i} \int_{\Gamma_{b}} e^{\lambda t} \lambda^{\alpha-1} R\left(\lambda^{\alpha} ; A_{n}\right) x_{n} d \lambda\right\}\right)
\end{aligned}
$$

где $\Gamma, \Gamma_{a}, \Gamma_{b}$ выбраны так же, как в теореме 5.2.1. Второе слагаемое можно сделать меньше произвольного $\varepsilon>0$. Первое слагаемое обращается в нуль в силу равностепенной непрерывности семейства $\left\{R\left(\lambda^{\alpha} ; A_{n}\right) x_{n}\right\}$ на $\Gamma_{a}$ (см. замечание 5.2.2). Таким образом, заключаем, что семейство $\left\{S_{\alpha}\left(t, A_{n}\right) x_{n}\right\}$ является $P$-компактным.

$(2) \Longrightarrow(1)$. Обратно, поскольку $S_{\alpha}\left(t, A_{n}\right) \stackrel{\mathcal{P P}}{\longrightarrow} S_{\alpha}(t, A)$ компактно для любого $t>0$, имеем $S_{\alpha}\left(t, A_{n}\right) \stackrel{\mathcal{P P}}{\longrightarrow} S_{\alpha}(t, A)$ и семейство $\left\{S_{\alpha}\left(t, A_{n}\right) x_{n}\right\}$ является $P$-компактным для любой последовательности $\left\{x_{n}\right\},\left\|x_{n}\right\|_{E_{n}}=O(1)$, и любого $t>0$. Очевидно, $R\left(\lambda^{\alpha} ; A_{n}\right) \stackrel{\mathcal{P P}}{\longrightarrow} R\left(\lambda^{\alpha} ; A\right)$. Действительно, нужно показать, что $\mu\left(\left\{R\left(\lambda^{\alpha} ; A_{n}\right) x_{n}\right\}\right)=0$ для любой последовательности $\left\{x_{n}\right\}$, $\left\|x_{n}\right\|_{E_{n}}=O(1)$. Имеем

$$
\begin{aligned}
& \mu\left(\left\{R\left(\lambda^{\alpha} ; A_{n}\right) x_{n}\right\}\right)= \\
& =\mu\left(\left\{\lambda^{1-\alpha} \int_{0}^{\infty} e^{-\lambda t} S_{\alpha}\left(t, A_{n}\right) x_{n} d t\right\}\right) \leqslant \mu\left(\left\{\lambda^{1-\alpha} \int_{0}^{q} e^{-\lambda t} S_{\alpha}\left(t, A_{n}\right) x_{n} d t\right\}\right)+ \\
& \quad+\mu\left(\left\{\lambda^{1-\alpha} \int_{Q}^{\infty} e^{-\lambda t} S_{\alpha}\left(t, A_{n}\right) x_{n} d t\right\}\right)+\mu\left(\left\{\lambda^{1-\alpha} \int_{q}^{Q} e^{-\lambda t} S_{\alpha}\left(t, A_{n}\right) x_{n} d t\right\}\right),
\end{aligned}
$$

где $q$ мало, а $Q$ достаточно велико. Первое и второе слагаемые меньше $\varepsilon$. Из замечания 5.2.1 известно, что семейство $\left\{S_{\alpha}\left(t, A_{n}\right)\right\}$ равномерно непрерывно на отрезке $[q, Q]$. Тогда третье слагаемое равно нулю. Доказательство завершено. 
Теорема 5.2.4 (см. [187]). Пусть $A$ и $A_{n}$ - генераторы аналитических $\alpha$-разрешающих семейств $S_{\alpha}(\cdot, A)$ и $S_{\alpha}\left(\cdot, A_{n}\right)$, соответственно. Предположим, что выполнены условия (A) и (B'), компактные резольвенты $R\left(\lambda^{\alpha} ; A_{n}\right)$ u $R\left(\lambda^{\alpha} ; A\right)$ сходятся компактно, т.е. $R\left(\lambda^{\alpha} ; A_{n}\right) \stackrel{\mathcal{P P}}{\longrightarrow}$ $R\left(\lambda^{\alpha} ; A\right)$ компактно для некоторого $\lambda^{\alpha} \in \rho(A)$ u $u_{n}^{0} \stackrel{\mathcal{P}}{\longrightarrow} u^{0}$. Предположим такэсе, что выполнены следующие условия:

(i) функции $f_{n}, f$ непрерывны по обоим аргументам и существует такал константа $\bar{M}$, не зависящая от $n$, что

$$
\sup _{\substack{t \in[0, T],\left\|x_{n}\right\|_{E_{n}} \leqslant 1}}\left\|f_{n}\left(t, x_{n}\right)\right\|_{E_{n}} \leqslant \bar{M}
$$

(ii) функиия $f(\cdot, \cdot)$ такова, что существует единственное обобщенное решение $u^{*}(t)$ задачи (5.1.1) на отрезке $[0, T]$ (например, $f(\cdot, \cdot)$ удовлетворяет условиям теоремы 5.2.1);

(iii) $f_{n}\left(t, x_{n}\right) \stackrel{\mathcal{P}}{\longrightarrow} f(t, x)$ равномерно по $t \in[0, T]$ при $x_{n} \stackrel{\mathcal{P}}{\longrightarrow} x$.

Тогда для почти всех $n$ задачи (5.1.5) имеют обобщенные решения $u_{n}^{*}(t), t \in[0, T]$, в окрестности точки $p_{n} u^{*}(t)$. Каждая последовательность $\left\{u_{n}^{*}(t)\right\}$ является $P$-компактной $u u_{n}^{*}(t) \stackrel{\mathcal{P}}{\longrightarrow} u^{*}(t)$ равномерно по $t \in[0, T]$.

Доказательство. Из теоремы 5.2 .3 получаем, что $S_{\alpha}\left(t, A_{n}\right) \stackrel{\mathcal{P P}}{\longrightarrow} S_{\alpha}(t, A)$ компактно для любого $t>0$. Действительно, известно, что

$$
\begin{gathered}
u(t)=S_{\alpha}(t, A) u^{0}+\int_{0}^{t} S_{\alpha}(t-s, A) f(s, u(s)) d s, \\
u_{n}(t)=S_{\alpha}\left(t, A_{n}\right) u_{n}^{0}+\int_{0}^{t} S_{\alpha}\left(t-s, A_{n}\right) f_{n}\left(s, u_{n}(s)\right) d s
\end{gathered}
$$

- обобщенные решения задач (5.1.1) и (5.1.5), соответственно. Положим

$$
\begin{gathered}
(K u)(t):=S_{\alpha}(t, A) u^{0}+\int_{0}^{t} S_{\alpha}(t-s, A) f(s, u(s)) d s, \\
\left(K_{n} u_{n}\right)(t):=S_{\alpha}\left(t, A_{n}\right) u_{n}^{0}+\int_{0}^{t} S_{\alpha}\left(t-s, A_{n}\right) f_{n}\left(s, u_{n}(s)\right) d s .
\end{gathered}
$$

Во-первых, покажем, что оператор $K: C([0, T] ; E) \rightarrow C([0, T] ; E)$ компактен. Пусть $\left\{u_{k}(\cdot)\right\}-$ множество таких функций $u_{k}(\cdot) \in C([0, T] ; E)$, что sup $\left\|u_{k}(t)\right\|=O(1)$. Из (i) следует, что функция $f(\cdot, \cdot)$ ограничена, так что множество функций $\left\{\left(K u_{k}\right)(\cdot)\right\}$ равномерно ограничено, где 
$\left(K u_{k}\right)(\cdot) \in C([0, T] ; E)$. Для $0<t_{1}<t_{2} \leqslant T$

$$
\begin{aligned}
& \left\|\left(K u_{k}\right)\left(t_{2}\right)-\left(K u_{k}\right)\left(t_{1}\right)\right\|= \\
& =\left\|S_{\alpha}\left(t_{2}, A\right) u_{k}^{0}-S_{\alpha}\left(t_{1}, A\right) u_{k}^{0}+\int_{0}^{t_{2}} S_{\alpha}\left(t_{2}-s, A\right) f\left(s, u_{k}(s)\right) d s-\int_{0}^{t_{1}} S_{\alpha}\left(t_{1}-s, A\right) f\left(s, u_{k}(s)\right) d s\right\| \leqslant \\
& \leqslant\left\|S_{\alpha}\left(t_{2}, A\right) u_{k}^{0}-S_{\alpha}\left(t_{1}, A\right) u_{k}^{0}\right\|+\int_{0}^{t_{1}-\delta}\left\|S_{\alpha}\left(t_{2}-s, A\right)-S_{\alpha}\left(t_{1}-s, A\right)\right\|\left\|f\left(s, u_{k}(s)\right)\right\| d s+ \\
& +\int_{t_{1}-\delta}^{t_{1}}\left\|S_{\alpha}\left(t_{2}-s, A\right)-S_{\alpha}\left(t_{1}-s, A\right)\right\|\left\|f\left(s, u_{k}(s)\right)\right\| d s+\int_{t_{1}}^{t_{2}}\left\|S_{\alpha}\left(t_{2}-s, A\right)\right\|\left\|f\left(s, u_{k}(s)\right)\right\| d s \leqslant \\
& \leqslant\left\|S_{\alpha}\left(t_{2}, A\right)-S_{\alpha}\left(t_{1}, A\right)\right\|+\bar{M}\left(t_{1}-\delta\right) \sup _{s \in\left[0, t_{1}-\delta\right]}\left\|S_{\alpha}\left(t_{2}-s, A\right)-S_{\alpha}\left(t_{1}-s, A\right)\right\|+ \\
& +2 M \bar{M} \delta+M \bar{M}\left(t_{2}-t_{1}\right) \rightarrow 0 \quad \text { при }\left|t_{2}-t_{1}\right| \rightarrow 0 .
\end{aligned}
$$

Это означает, что $\left\{\left(K u_{k}\right)(\cdot)\right\}$ равномерно непрерывно. Докажем, что последовательность $\left\{\left(K u_{k}\right)(t)\right\}$ относительно компактна для любого $t>0$. Из теоремы 5.2 .2 следует, что $S_{\alpha}(t, A)$ компактно для любого $t>0$. Выберем произвольно $0<\varepsilon<t$; тогда $S_{\alpha}(\varepsilon, A)$ компактно,

$$
\left\{S_{\alpha}(\varepsilon, A) \int_{0}^{t-\varepsilon} S_{\alpha}(t-s-\varepsilon, A) f\left(s, u_{k}(s)\right) d s\right\}
$$

относительно компактно для любого $t>0$. Тогда

$$
\begin{aligned}
&\left\|S_{\alpha}(\varepsilon, A) \int_{0}^{t-\varepsilon} S_{\alpha}(t-s-\varepsilon, A) f\left(s, u_{k}(s)\right) d s-\int_{0}^{t-\varepsilon} S_{\alpha}(t-s, A) f\left(s, u_{k}(s)\right) d s\right\| \leqslant \\
& \leqslant \int_{0}^{t-\varepsilon}\left\|S_{\alpha}(\varepsilon, A) S_{\alpha}(t-s-\varepsilon, A)-S_{\alpha}(t-s, A)\right\|\left\|f\left(s, u_{k}(s)\right)\right\| d s \leqslant \\
& \leqslant \int_{0}^{t-\varepsilon-\delta}\left\|S_{\alpha}(\varepsilon, A) S_{\alpha}(t-s-\varepsilon, A)-S_{\alpha}(t-s, A)\right\|\left\|f\left(s, u_{k}(s)\right)\right\| d s+ \\
&+\int^{t-\varepsilon}\left\|S_{\alpha}(\varepsilon, A) S_{\alpha}(t-s-\varepsilon, A)-S_{\alpha}(t-s, A)\right\|\left\|f\left(s, u_{k}(s)\right)\right\| d s \leqslant \int_{t-\varepsilon-\delta}^{t-\varepsilon} d s \rightarrow 0
\end{aligned}
$$


при $\delta \rightarrow 0$ и $\varepsilon \rightarrow 0$. Предел первого слагаемого, равный нулю, получим при помощи [109, лемма 3.4]. Кроме того, при $\varepsilon \rightarrow 0$ имеем

$$
\begin{aligned}
& \left\|S_{\alpha}(\varepsilon, A) \int_{0}^{t-\varepsilon} S_{\alpha}(t-s-\varepsilon, A) f\left(s, u_{k}(s)\right) d s-\int_{0}^{t} S_{\alpha}(t-s, A) f\left(s, u_{k}(s)\right) d s\right\| \leqslant \\
& \leqslant\left\|S_{\alpha}(\varepsilon, A) \int_{0}^{t-\varepsilon} S_{\alpha}(t-s-\varepsilon, A) f\left(s, u_{k}(s)\right) d s-\int_{0}^{t-\varepsilon} S_{\alpha}(t-s, A) f\left(s, u_{k}(s)\right) d s\right\|+ \\
& \leqslant\left\|S_{\alpha}(\varepsilon, A) \int_{0}^{t-\varepsilon} S_{\alpha}(t-s-\varepsilon, A) f\left(s, u_{k}(s)\right) d s-\int_{0}^{t-\varepsilon} S_{\alpha}(t-s, A) f\left(s, u_{k}(s)\right) d s\right\| \leqslant
\end{aligned}
$$

Это означает, что

$$
\left\{\int_{0}^{t} S_{\alpha}(t-s, A) f\left(s, u_{k}(s)\right) d s\right\}
$$

относительно компактно для любого $t>0$. Очевидно, при $t=0$ это множество также относительно компактно. Поэтому для любого $t$, множество $\left\{\left(K u_{k}\right)(t)\right\}$ относительно компактно в $E$. Тогда из обобщеннной теоремы Арцела-Асколи вытекает, что $\left\{\left(K u_{k}\right)(t)\right\}$ - компактное множество в $E$ и поэтому $\left\{\left(K u_{k}\right)(\cdot)\right\}$ компактно в $C([0, T] ; E)$. Таким образом, $K$-компактный оператор. Аналогично доказывается, что операторы $K_{n}$ также компактны.

Если $u_{n}(\cdot) \stackrel{\mathcal{P}}{\longrightarrow} u(\cdot)$, то из условия (iii) следует, что $f_{n}\left(t, u_{n}(t)\right) \stackrel{\mathcal{P}}{\longrightarrow} f(t, u(t))$, равномерно по $t \in[0, T]$. Получаем

$$
\begin{aligned}
\sup _{t \in[0, T]}\left\|\left(K_{n} u_{n}\right)(t)-p_{n}(K u)(t)\right\|_{E_{n}} \leqslant \sup _{t \in[0, T]}\left\|S_{\alpha}\left(t, A_{n}\right) u_{n}^{0}-p_{n} S_{\alpha}(t, A) u^{0}\right\|_{E_{n}}+ \\
+\sup _{t \in[0, T]}\left\|\int_{0}^{t} S_{\alpha}\left(t-s, A_{n}\right) f_{n}\left(s, u_{n}(s)\right) d s-p_{n} \int_{0}^{t} S_{\alpha}(t-s, A) f(s, u(s)) d s\right\|_{E_{n}} \rightarrow 0 .
\end{aligned}
$$

Таким образом, $K_{n} \stackrel{\mathcal{P P}}{\longrightarrow} K$. Рассмотрим произвольную последовательность $\left\{u_{n}(\cdot)\right\}$ функ-

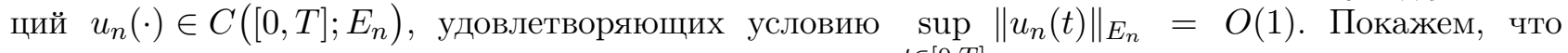
$\mu\left(\left\{\left(K_{n} u_{n}\right)(t)\right\}\right)=0$ для всех $t \in[0, T]$. Действительно,

$$
\begin{aligned}
\mu\left(\left\{\left(K_{n} u_{n}\right)(t)\right\}\right) \leqslant \mu\left(\left\{S_{\alpha}\left(t, A_{n}\right) u_{n}^{0}\right\}\right)+\mu\left(\left\{\int_{0}^{t} S_{\alpha}\left(t-s, A_{n}\right) f_{n}\left(s, u_{n}(s)\right) d s\right\}\right) \leqslant \\
\leqslant \mu\left(\left\{S_{\alpha}\left(t, A_{n}\right) u_{n}^{0}\right\}\right)+\mu\left(\left\{\int_{0}^{t-\delta} S_{\alpha}\left(t-s, A_{n}\right) f_{n}\left(s, u_{n}(s)\right) d s\right\}\right)+ \\
\quad+\sup _{t \in[0, T]}\left\|\int_{t-\delta}^{t} S_{\alpha}\left(t-s, A_{n}\right) f_{n}\left(s, u_{n}(s)\right) d s\right\|_{E_{n}} .
\end{aligned}
$$

Очевидно, первый член равен нулю. Поскольку

$$
\left\|S_{\alpha}\left(t, A_{n}\right)\right\|_{B\left(E_{n}\right)} \leqslant M e^{\omega t}, \quad \sup _{t \in[0, T]}\left\|f_{n}\left(t, u_{n}(t)\right)\right\|_{E_{n}} \leqslant \bar{M}
$$


заключаем, что третий член можно сделать меньше произвольного $\varepsilon$, выбрав достаточно малое $\delta$. Из леммы 5.2.1 и условия (iii) следует, что семейство $\left\{f_{n}\left(s, u_{n}(s)\right)\right\}$ равностепенно непрерывно на $[0, t-\delta]$. Известно также, что семейство $\left\{S_{\alpha}\left(t-s, A_{n}\right)\right\}$ также равностепенно непрерывно на $[0, t-\delta]$. Таким образом,

$$
\mu\left(\left\{\int_{0}^{t-\delta} S_{\alpha}\left(t-s, A_{n}\right) f_{n}\left(s, u_{n}(s)\right) d s\right\}\right)=0 .
$$

Поэтому семейство $\left\{\left(K_{n} u_{n}\right)(\cdot)\right\}$ является $P$-компактным и, следовательно, $K_{n} \stackrel{\mathcal{P} \mathcal{P}}{\longrightarrow} K$ компактHO.

Из предположения следует, что $K$ не имеет неподвижных точек на границе $\partial S_{r}$, где

$$
S_{r}=\left\{u: \sup _{t \in[0, T]}\left\|u(t)-u^{*}(t)\right\|<r\right\} .
$$

Из результатов работы [259] следует, что $\gamma\left(I-K ; \partial S_{r}\right)=\gamma\left(I_{n}-K_{n} ; \partial S_{n, r}\right)$ при $n \geqslant n_{0}$. Если $\gamma\left(I-K ; \partial S_{r}\right) \neq 0$, то из [259, теорема 3] будет следовать, что решения задач $(5.1 .5)$ существуют в окрестности точки $p_{n} u^{*}(t)$, каждая последовательность $\left\{u_{n}^{*}(t)\right\}$ является $P$-компактной и $u_{n}^{*}(t) \stackrel{\mathcal{P}}{\longrightarrow} u^{*}(t)$ равномерно по $t \in[0, T]$. Отсюда вытекает требуемый результат.

Поскольку семейство $S_{\alpha}(\cdot, A)$ аналитично и компактно, доказательство того факта, что $\gamma\left(I-K ; \partial S_{r}\right)=1$, буквально повторяет доказательство [262, теорема 14.2.3].

\section{3. СЛУЧАЙ КЛАССИЧЕСКОЙ ПРАВОЙ ЧАСТИ}

В этом разделе будет рассмотрена аппроксимация корректно поставленной задачи Коши

$$
\left(\mathcal{D}_{t}^{\alpha} u\right)(t)=A u(t)+f(t, u(t)), \quad 0<t \leqslant T ; \quad u(0)=u^{0}
$$

где $\mathcal{D}_{t}^{\alpha}$ - производная Капуто-Джрбашяна, оператор $A$ порождает аналитическое и компактное $\alpha$-разрешающее семейство $S_{\alpha}(\cdot, A)$, а функция $f(\cdot, \cdot)$ является достаточно гладкой по обоим аргументам.

Определение 5.3.1 (см. [152]). Семейство $\left\{P_{\alpha}(t, A)\right\}_{t \geqslant 0}$ сильно непрерывных функций $P_{\alpha}(\cdot, A):(0, \infty) \longrightarrow B(E)$ называется $(\alpha, \alpha)$-разрешающим семейством, порожденньм оператором $A$, если существует такое $\omega \geqslant 0$, что $\left\{\lambda^{\alpha}: \operatorname{Re} \lambda>\omega\right\} \subset \rho(A)$ и

$$
\left(\lambda^{\alpha} I-A\right)^{-1} x=\int_{0}^{\infty} e^{-\lambda t} P_{\alpha}(t, A) x d t, \quad \operatorname{Re} \lambda>\omega, \quad x \in E .
$$

Замечание 5.3.1 (см. [66,152,173]). При $1<\alpha<2$ очевидно, что если оператор $A$ порождает $\alpha$-разрешающее семейство $S_{\alpha}(t, A)$, то он также является генератором $(\alpha, \alpha)$-разрешающего семейства $P_{\alpha}(t, A)$ и

$$
P_{\alpha}(t, A)=\left(g_{\alpha-1} * S_{\alpha}\right)(t)
$$

В случае $0<\alpha<1$, если оператор $A$ порождает аналитическое $\alpha$-разрешающее семейство $S_{\alpha}(t, A)$, то он также является генератором аналитического $(\alpha, \alpha)$-разрешающего семейства

$$
P_{\alpha}(t, A)=\frac{1}{2 \pi i} \int_{\Gamma} e^{\lambda t} R\left(\lambda^{\alpha} ; A\right) d \lambda
$$

и

$$
\left(g_{1-\alpha} * P_{\alpha}\right)(t)=S_{\alpha}(t, A)
$$


Семейство $P_{\alpha}(\cdot, A)$, обладает следующими свойствами (см. $\left.[152,173]\right)$ :

$$
\begin{aligned}
& P_{\alpha}(t, A) x=g_{\alpha}(t) x+A \int_{0}^{t} g_{\alpha}(t-s) P_{\alpha}(s, A) x d s, \quad t>0, \quad \text { для любого } x \in E, \\
& A P_{\alpha}(t, A) x=P_{\alpha}(t, A) A x \quad \text { для любого } x \in D(A) .
\end{aligned}
$$

В этом разделе мы рассматриваем только случай корректно поставленной задачи Коши для дифференциального уравнения порядка $0<\alpha<1$. В этом случае справедлива следующая лемма.

Лемма 5.3.1 (см. [173]). Пусть A-генератор аналитического $\alpha$-разрешающего семейства $S_{\alpha}(t, A)$. Справедливы следующие утверждения:

(1) $P_{\alpha}(t, A) \in B(E) u$

$$
\left\|P_{\alpha}(t, A)\right\| \leqslant M e^{\omega t}\left(1+t^{\alpha-1}\right) \quad \text { для любого } t>0 ;
$$

(2) $P_{\alpha}(t, A) x \in D(A)$ для любого $x \in E$ u

$$
\left\|A P_{\alpha}(t, A)\right\| \leqslant M e^{\omega t}\left(1+t^{-1}\right) \quad \text { для любого } t>0
$$

(3) $S_{\alpha}^{\prime}(t, A)=A P_{\alpha}(t, A)$ для любого $t>0, R\left(P_{\alpha}^{(l)}(t, A)\right) \subseteq D(A)$ для любого иелого $l \geqslant 0$ и

$$
\left\|A^{k} P_{\alpha}^{(l)}(t, A)\right\| \leqslant M e^{\omega t}\left(1+t^{-l-1-\alpha(k-1)}\right) \quad \text { для любого } t>0,
$$

где $k=0,1$.

Определение 5.3.2. Функция $u(\cdot) \in C([0, T] ; E)$ называется обобщенным решением задачи (5.3.1), если она удовлетворяет уравнению

$$
u(t)=S_{\alpha}(t, A) u^{0}+\int_{0}^{t} P_{\alpha}(t-s, A) f(s, u(s)) d s .
$$

Теорема 5.3.1 (см. $[109,175,192,224])$. Пусть $A$ - генератор аналитического $\alpha$-разрешающего семейства $S_{\alpha}(\cdot, A)$ на банаховом пространстве E. Предположим, что функиия $f(\cdot, \cdot)$ : $[0, T] \times E \rightarrow E$ непрерьвна по обоим аргументам и существует такая константа $L>0$, что

$$
\|f(t, x)-f(t, y)\| \leqslant L\|x-y\| \quad \text { npu } t \in[0, T] u x, y \in E .
$$

Тогда существует единственное обобщенное решение $u(\cdot) \in C([0, T] ; E)$ задачи (5.3.1). Кроме того, отображение $u(0) \rightarrow u(\cdot)$ из $E$ в $C([0, T] ; E)$ является непрерывным по Липшичу.

Доказательство. Рассмотрим оператор

$$
K: C([0, T] ; E) \rightarrow C([0, T] ; E), \quad(K u)(t):=S_{\alpha}(t, A) u^{0}+\int_{0}^{t} P_{\alpha}(t-s, A) f(s, u(s)) d s, \quad t \in[0, T] .
$$

Чтобы доказать, что задача (5.3.1) имеет единственное обобщенное решение, нужно убедиться, что оператор $K$ имеет неподвижную точку. Пусть $u, v \in C([0, T] ; E)$; тогда из условий на функцию $f(\cdot, \cdot)$ следует, что

$$
\begin{gathered}
\|(K u)(t)-(K v)(t)\| \leqslant \int_{0}^{t}\left\|P_{\alpha}(t-s, A)\right\| \cdot\|f(s, u(s))-f(s, v(s))\| d s \leqslant \\
\leqslant M L \int_{0}^{t} g_{\alpha}(t-s)\|u(s)-v(s)\| d s \leqslant M L t^{\alpha}\|u-v\|_{C([0, T] ; E)} .
\end{gathered}
$$

Таким образом,

$$
\sup _{t \in[0, T]}\left\|\left(K^{n} u\right)(t)-\left(K^{n} v\right)(t)\right\| \leqslant \sup _{t \in[0, T]} \frac{\left(M L t^{\alpha}\right)^{n}}{n !}\|u-v\|_{C([0, T] ; E)} \leqslant \frac{\left(M L T^{\alpha}\right)^{n}}{n !}\|u-v\|_{C([0, T] ; E)} .
$$


Поскольку $\left(M L T^{\alpha}\right)^{n} / n !<1$ при достаточно больших $n$, оператор $K$ является сжимающим. По теореме о сжимающих отображениях заключаем, что $K$ имеет единственную неподвижную точку, которая и является решением задачи (5.3.1).

Пусть $v(\cdot)$ - обобщенн'мягк'ое решение задачи (5.3.1); тогда

$$
\begin{aligned}
\|u(t)-v(t)\| \leqslant\left\|S_{\alpha}(t, A) u(0)-S_{\alpha}(t, A) v(0)\right\| & +\int_{0}^{t}\left\|P_{\alpha}(t-s, A)\right\| \cdot\|f(s, u(s))-f(s, v(s))\| d s \leqslant \\
& \leqslant M\left\|u^{0}-v^{0}\right\|+M L \int_{0}^{t} g_{\alpha}(t-s)\|u(s)-v(s)\| d s .
\end{aligned}
$$

Согласно неравенству Гронуолла

$$
\|u(t)-v(t)\| \leqslant M e^{M L T^{\alpha}}\|u(0)-v(0)\| .
$$

Это означает, что решение $u(\cdot)$ единственно, а отображение $u(0) \rightarrow u(\cdot)$ липшиц-непрерывно.

Заметим, задачи типа (5.3.1) и $(*)($ см. с. 5$)$ в случае, когда $\exp (\cdot A)$ не компактна, но является уплотняющей, рассматривались в $[50,63,86,146,206,207]$.

\section{4. ПОЛУДИСКРЕТНАЯ АППРОКСИМАЦИЯ В КЛАССИЧЕСКОМ СЛУЧАЕ}

Полудискретная аппроксимация в рамках общей дискретизационной схемы для задачи (5.3.1) осуществляется следующими задачами Коши в банаховых пространствах $E_{n}$ :

$$
\left(\mathcal{D}_{t}^{\alpha} u_{n}\right)(t)=A_{n} u_{n}(t)+f_{n}\left(t, u_{n}(t)\right), \quad 0<t \leqslant T, \quad u_{n}(0)=u_{n}^{0},
$$

здесь операторы $A_{n}$ порождают аналитические и компактные $\alpha$-разрешающие семейства $S_{\alpha}\left(\cdot, A_{n}\right)$, а функции $f_{n}(\cdot, \cdot)$ являются достаточно гладкими.

Замечание 5.4.1. Из условия $\left(\mathrm{C}^{\prime}\right)$ следует, что

$$
\sup _{t \in[0, T]}\left\|S_{\alpha}\left(t, A_{n}\right) x_{n}-p_{n} S_{\alpha}(t, A) x\right\|_{E_{n}} \rightarrow 0 \quad \text { при } n \rightarrow \infty
$$

если $x_{n} \stackrel{\mathcal{P}}{\longrightarrow} x, x_{n} \in E_{n}, x \in E$. Тогда из уравнения (5.3.4) получаем, что

$$
\begin{gathered}
\sup _{t \in[0, T] \|}\left\|\int_{0}^{t} P_{\alpha}\left(t-s, A_{n}\right) x_{n} d s-p_{n} \int_{0}^{t} P_{\alpha}(t-s, A) x d s\right\|_{E_{n}}= \\
=\sup _{t \in[0, T]}\left\|\left(g_{1} * P_{\alpha}\right)\left(t, A_{n}\right) x_{n}-p_{n}\left(g_{1} * P_{\alpha}\right)(t, A) x\right\|_{E_{n}}= \\
=\sup _{t \in[0, T]}\left\|\left(g_{\alpha} * g_{1-\alpha} * P_{\alpha}\right)\left(t, A_{n}\right) x_{n}-p_{n}\left(g_{\alpha} * g_{1-\alpha} * P_{\alpha}\right)(t, A) x\right\|_{E_{n}}= \\
=\sup _{t \in[0, T]}\left\|\int_{0}^{t} g_{\alpha}(t-s) S_{\alpha}\left(s, A_{n}\right) x_{n} d s-p_{n} \int_{0}^{t} g_{\alpha}(t-s) S_{\alpha}(s, A) x d s\right\|_{E_{n}}= \\
=\sup _{t \in[0, T] \|}\left\|\int_{0}^{t} g_{\alpha}(t-s)\left(S_{\alpha}\left(s, A_{n}\right) x_{n}-p_{n} S_{\alpha}(s, A) x\right) d s\right\|_{E_{n}} \leqslant \\
\leqslant \int_{0}^{t}\left|g_{\alpha}(t-s)\right| d s \sup _{t \in[0, T]}\left\|S_{\alpha}\left(s, A_{n}\right) x_{n}-p_{n} S_{\alpha}(s, A) x\right\|_{E_{n}}=
\end{gathered}
$$


если $x_{n} \stackrel{\mathcal{P}}{\longrightarrow} x, x_{n} \in E_{n}, x \in E$.

Замечание 5.4.2. В силу представления

$$
S_{\alpha}\left(z, A_{n}\right)=\frac{1}{2 \pi i} \int_{\Gamma} e^{\lambda z} \lambda^{\alpha-1} R\left(\lambda^{\alpha} ; A_{n}\right) d \lambda,
$$

где $\Gamma$ - положительно ориентированный контур, являющийся границей множества $\omega+\Sigma_{\beta+\pi / 2}$, получаем равностепенную непрерывность семейства $\left\{S_{\alpha}\left(z, A_{n}\right)\right\}$ на $\Sigma(\beta) \cap\{z: \operatorname{Re} z \leqslant r\} \backslash\{0\}$. Поскольку равностепенная непрерывность семейства $\left\{S_{\alpha}\left(z, A_{n}\right)\right\}$ при $z=0$ была доказана ранее (см. [186, теорема 7]), заключаем, что семейство $\left\{S_{\alpha}\left(z, A_{n}\right)\right\}$ равностепенно непрерывно на $\Sigma(\beta) \cap$ $\{z: \operatorname{Re} z \leqslant r\}$.

Лемма 5.4.1. Пусть $S_{\alpha}(t, A), t \geqslant 0,-$ аналитическое $\alpha$-разрешающее семейство, порожденное оператором $A$, в банаховом пространстве $E$. Тогда $\alpha$-разрешающее $u(\alpha, \alpha)$-разрешающее семейства $S_{\alpha}(t, A)$ и $P_{\alpha}(t, A)$ непрерывны в равномерной операторной топологии для всех $t>0$.

Доказательство. Из [109, лемма 3.8] известно, что $\alpha$-разрешающее семейство $S_{\alpha}(\cdot, A)$ непрерывно в равномерной операторной топологии для всех $t>0$. Из замечания 5.3 .1 получим, что $(\alpha, \alpha)$ разрешающее семейство $P_{\alpha}(t, A)$, порожденное оператором $A$, аналитично. Остается показать, что $P_{\alpha}(t, A)$ непрерывно в равномерной операторной топологии для всех $t>0$.

Действительно, из леммы 5.3.1 следует, что

$$
\left\|P_{\alpha}^{\prime}(t, A)\right\| \leqslant M\left(1+t^{\alpha-2}\right) .
$$

Тогда для любых $x \in E,\|x\| \leqslant 1$, и $|h|<t$ имеем

$$
\begin{aligned}
& \left\|P_{\alpha}(t+h, A) x-P_{\alpha}(t, A) x\right\|= \\
& =\left\|\int_{t}^{t+h} P_{\alpha}^{\prime}(s, A) x d s\right\| \leqslant M\|x\| \cdot\left\|\int_{t}^{t+h} s^{\alpha-2} d s\right\| \leqslant M \frac{(t+h)^{\alpha-1}-t^{\alpha-1}}{\alpha-1} \rightarrow 0 \quad \text { при } h \rightarrow 0 .
\end{aligned}
$$

Лемма доказана.

Теорема 5.4.1 (см. [192]). Пусть $S_{\alpha}(\cdot, A)$-аналитическое $\alpha$-разрешающее семейство, порожденное оператором $A$, в банаховом пространстве $E$. Предположим, что семейства $S_{\alpha}(t, A)$, $P_{\alpha}(t, A)$ компактны для любого $t>0$. Тогда

$$
\lim _{h \rightarrow 0}\left\|P_{\alpha}(t, A)-S_{\alpha}(h, A) P_{\alpha}(t-h, A)\right\|=0 \quad \text { для любого } t>0 .
$$

Доказательство. Из леммы 5.4.1 следует, что $\alpha$-разрешающее и $(\alpha, \alpha)$-разрешающее семейства $S_{\alpha}(t, A)$ и $P_{\alpha}(t, A)$ непрерывны в равномерной операторной топологии для всех $t>0$. Пусть $t>0$ и $0<h<t$. Сначала докажем, что для любого $t>0$

$$
\lim _{h \rightarrow 0}\left\|P_{\alpha}(t+h, A)-S_{\alpha}(h, A) P_{\alpha}(t, A)\right\|=0
$$

Выберем $\varepsilon>0$. Поскольку семейство $P_{\alpha}(t, A)$ компактно, множество $P_{t}:=\left\{P_{\alpha}(t, A) x:\|x\| \leqslant 1\right\}$ также компактно. Тогда для любого $x \in E,\|x\| \leqslant 1$, существует такая конечная $\varepsilon$-сеть

$$
\left\{P_{\alpha}(t, A) x_{1}, P_{\alpha}(t, A) x_{2}, \ldots, P_{\alpha}(t, A) x_{l}\right\} \subset P_{t}
$$

что

$$
\left\|P_{\alpha}(t, A) x-P_{\alpha}(t, A) x_{i}\right\| \leqslant \frac{\varepsilon}{3(M+1)}, \quad 1 \leqslant i \leqslant l .
$$

Из сильной непрерывности $S_{\alpha}(h, A) x_{i} \rightarrow x_{i}$ при $h \rightarrow 0$ для всех $i=1, \ldots l$, следует, что существует такое $0<h_{1}<t$, что

$$
\left\|P_{\alpha}(t, A) x_{i}-S_{\alpha}(h, A) P_{\alpha}(t, A) x_{i}\right\| \leqslant \frac{\varepsilon}{3} \quad \text { для всех } 0 \leqslant h \leqslant h_{1} \text { и } 1 \leqslant i \leqslant l .
$$


Из непрерывности семейства $P_{\alpha}(t, A)$ в равномерной операторной топологии следует, что существует такое $0<h_{2}<t$, что

$$
\left\|P_{\alpha}(t, A) x-P_{\alpha}(t+h, A) x\right\| \leqslant \frac{\varepsilon}{3} \quad \text { для всех } 0 \leqslant h \leqslant h_{2} \text { и }\|x\| \leqslant 1 .
$$

Следовательно, для любого $0 \leqslant h \leqslant \min \left\{h_{1}, h_{2}\right\}$ и $\|x\| \leqslant 1$ из уравнений (5.4.3), (5.4.4), (5.4.5) следует, что

$$
\begin{aligned}
& \left\|P_{\alpha}(t+h, A) x-S_{\alpha}(h, A) P_{\alpha}(t, A) x\right\| \leqslant \\
& \leqslant\left\|P_{\alpha}(t+h, A) x-P_{\alpha}(t, A) x\right\|+\left\|P_{\alpha}(t, A) x-P_{\alpha}(t, A) x_{i}\right\|+ \\
& +\left\|P_{\alpha}(t, A) x_{i}-S_{\alpha}(h, A) P_{\alpha}(t, A) x_{i}\right\|+\left\|S_{\alpha}(h, A) P_{\alpha}(t, A) x_{i}-S_{\alpha}(h, A) P_{\alpha}(t, A) x\right\| \leqslant \\
& \leqslant\left\|P_{\alpha}(t+h, A) x-P_{\alpha}(t, A) x\right\|+(M+1)\left\|P_{\alpha}(t, A) x-P_{\alpha}(t, A) x_{i}\right\|+ \\
& +\left\|P_{\alpha}(t, A) x_{i}-S_{\alpha}(h, A) P_{\alpha}(t, A) x_{i}\right\| \leqslant \varepsilon .
\end{aligned}
$$

Это означает, что предельное соотношение (5.4.2) выполняется равномерно при $t>0$. С другой стороны,

$$
\begin{gathered}
\left\|P_{\alpha}(t, A)-S_{\alpha}(h, A) P_{\alpha}(t-h, A)\right\| \leqslant\left\|P_{\alpha}(t, A)-P_{\alpha}(t+h, A)\right\|+ \\
+\left\|P_{\alpha}(t+h, A)-S_{\alpha}(h, A) P_{\alpha}(t, A)\right\|+\left\|S_{\alpha}(h, A) P_{\alpha}(t, A)-S_{\alpha}(h, A) P_{\alpha}(t-h, A)\right\| \leqslant \\
\leqslant\left\|P_{\alpha}(t, A)-P_{\alpha}(t+h, A)\right\|+\left\|P_{\alpha}(t+h, A)-S_{\alpha}(h, A) P_{\alpha}(t, A)\right\|+ \\
\quad+M\left\|P_{\alpha}(t, A)-P_{\alpha}(t-h, A)\right\| .
\end{gathered}
$$

Из непрерывности $(\alpha, \alpha)$-разрешающего семейства в равномерной операторной топологии заключаем, что

$$
\lim _{h \rightarrow 0}\left\|P_{\alpha}(t, A)-P_{\alpha}(t+h, A)\right\|=0, \quad \lim _{h \rightarrow 0} M\left\|P_{\alpha}(t, A)-P_{\alpha}(t-h, A)\right\|=0 .
$$

Таким образом, требуемое утверждение вытекает из (5.4.2). Теорема доказана.

В работах $[1,109]$ была впервые предпринята попытка характеризации компактности разрешающих семейств через компактность резольвент. В $[187,195]$ получен следующий результат.

Теорема 5.4.2. Пусть $A$ и $A_{n}$-генераторь аналитических $\alpha$-разрешающих семейств $S_{\alpha}(\cdot, A)$ и $S_{\alpha}\left(\cdot, A_{n}\right)$, соответственно. Предположим, что выполнены условия $(\mathrm{A})$ и $\left(\mathrm{B}^{\prime}\right)$, компактные резольвенты $R\left(\lambda^{\alpha} ; A_{n}\right)$ сходятся компактно $\kappa R\left(\lambda^{\alpha} ; A\right)$, т.е. $R\left(\lambda^{\alpha} ; A_{n}\right) \stackrel{\mathcal{P P}}{\longrightarrow} R\left(\lambda^{\alpha} ; A\right)$ компактно для некоторого $\lambda^{\alpha} \in \rho(A)$ u $u_{n}^{0} \stackrel{\mathcal{P}}{\longrightarrow} u^{0}$. Предположим также, что выполнены следующие условия:

(i) функиии $f_{n}$ u $f$ непрерывны по обоим аргументам и существует такая не зависящая от $n$ константа $\bar{M}$, что

$$
\sup _{\substack{t \in[0, T],\left\|x_{n}\right\|_{E_{n}} \leqslant 1}}\left\|f_{n}\left(t, x_{n}\right)\right\|_{E_{n}} \leqslant \bar{M} ;
$$

(ii) функиия $f(\cdot, \cdot)$ такова, что существует единственное обобщенное решение $u^{*}(t)$ задачи (5.3.1) на отрезке $[0, T]$ (например, $f(\cdot, \cdot)$ удовлетворяет условиям теоремы 5.3.1);

(iii) $f_{n}\left(t, x_{n}\right) \stackrel{\mathcal{P}}{\longrightarrow} f(t, x)$ равномерно по $t \in[0, T]$ nри $x_{n} \stackrel{\mathcal{P}}{\longrightarrow} x$.

Тогда для почти всех $n$ задачи (5.4.1) имеют обобщенные решения $u_{n}^{*}(t), t \in[0, T], \quad$ окрестности точки $p_{n} u^{*}(t)$. Каждая последовательность $\left\{u_{n}^{*}(t)\right\}$ является $P$-компактной $u$ $u_{n}^{*}(t) \stackrel{\mathcal{P}}{\longrightarrow} u^{*}(t)$ равномерно по $t \in[0, T]$. 
Доказательство теоремы 5.4.2 аналогично доказательству теоремы 5.2.4, но основано на теореме 5.4.1.

\section{5. Коэрцитивность в Пространствах $C_{0}^{\alpha}([0, T] ; E)$}

Свойство коэрцитивности (максимальной регулярности) играет ключевую роль в изучении различных типов дифференциальных уравнений с частными производными (см. [32-35, 60]). Это свойство особенно важно при изучении линеаризаций нелинейных уравнений. Рассмотрим следующую задачу Коши в абстрактном пространстве $E$ :

$$
\left\{\begin{array}{l}
u^{\prime}(t)=A u(t)+f(t), \quad 0 \leqslant t \leqslant T, \\
u(0)=u^{0}
\end{array}\right.
$$

где оператор $A$ является генератором $C_{0}$-полугруппы $\exp (t A), t \geqslant 0$. Имеется обширная литерату$\mathrm{pa}$, посвященная коэрцитивности задачи (5.5.1) в различных пространствах (см. [26]). Например, в [104] было доказано, что, вообще говоря, задача (5.5.1) не обладает свойством коэрцитивности в $C([0, T] ; E)$, за исключением случая, когда либо оператор $A$ непрерывен, либо пространства $E$ содержат подпространство, изоморфное пространству $c_{0}$ последовательностей, сходящихся к нулю. В [60] было указано, что в пространстве Гельдера $C_{0}^{\gamma}([0, T] ; E)$ аналитичность $C_{0}$-полугруппы эквивалентна коэрцитивности задачи (5.5.1). Более подробное изложение результатов о коэрцитивности задачи (5.5.1) в различных пространствах можно найти в $[26,60,127]$.

В то же время имеется обширная литература, посвященная коэрцитивности для дифференциальных уравнений второго порядка. В [57] рассматривалась слабая коэрцитивность для гиперболических дифференциальных уравнений второго порядка в пространствах $C^{\gamma}([0, T] ; E)$, $C\left([0, T] ; E^{\theta}\right)$ и $L^{p}\left([0, T] ; E^{\theta}\right)$, где $E^{\theta}$ - интерполяционное пространство. В [56] представлены разностные схемы для эллиптических задач в $L_{\tau_{n}}^{p}\left([0, T] ; E_{n}\right)$ и исследованы вопросы дискретной коэрцитивности. Коэрцитивность в $L^{p}([0, T] ; E)$ изучалась также в [59] (см. подробности в $\left.[26,58,262]\right)$.

Известно, что дробные дифференциальные уравнения стали одной из наиболее интересных тем исследований в связи с их широким применением в физике, технике, биологии и т. д. Многие полезные приложения по этим проблемам можно найти, например, в $[?, 26,66,109,143,155,173,176$, $183,186,189,223,270]$. Кроме того, изучались вопросы коэрцитивности решений дробных эволюционных уравнений. Например, в [66] рассматривались приложения дробных задач Коши в теории разрешающих операторов и анализировалась коэрцитивность в $L^{p}$ для дробных дифференциальных уравнений с производной Римана-Лиувилля и положительными и $\mathcal{R}$-секториальными операторами $A$. Коэрцитивность решений изучалась также в $[92,93]$. Позднее в [173] была рассмотрена регулярность по Гельдеру (не коэрцитивность) для дробных задач Коши с показателем из $(0,1)$; кроме того, результаты были обобщены на случай дифференциальных уравнений первого порядка (5.5.1) (см. [216]).

Отметим также, что при помощи метода операторнозначных мультипликаторов Фурье (см. [8285]) можно охарактеризовать корректность и коэрцитивность для вырожденных дифференциальных уравнений второго и дробного порядков в различных функциональных пространствах.

Теория приближений для дифференциальных уравнений в абстрактных пространствах была разработана в $[17,26,35,36,60,86,89,127,129,162,186-189,259,262]$. Изучались численные задачи для однородных дифференциальных уравнений и полулинейных дифференциальных уравнений. Так, аппроксимации для дробных дифференциальных уравнений в абстрактных пространствах рассматривались в [186-189], где были получены результаты о существовании и устойчивости решений и порядке сходимости разностных схем для общих аппроксимаций дробных задач Коши.

Мы рассмотрим корректность и коэрцитивность в пространстве Гельдера $C_{0}^{\gamma}([0, T] ; E)$ и полную дискретизацию для следующей неоднородной дробной задачи Коши в абстрактном пространстве $E$ :

$$
\left\{\begin{array}{l}
D^{\alpha} u(t)=A u(t)+f(t), \quad 0 \leqslant t \leqslant T, \\
u(0)=u^{0}
\end{array}\right.
$$

здесь оператор $A$ является генератором аналитического $\alpha$-разрешающего семейства, а $D^{\alpha}$ - дробная производная Капуто, $\alpha \in(0,1)$. 
5.5.1. Постановка задачи. Пусть $\gamma \in(0,1), T>0$ и $C^{\gamma}([0, T] ; E)$ - банахово пространство, состоящее из непрерывных функций $u(\cdot)$, заданных на отрезке $[0, T]$ и принимающих значения в банаховом пространстве $E$, снабженное следующей нормой:

$$
\|u(\cdot)\|_{C^{\gamma}([0, T] ; E)}=\sup _{0 \leqslant t \leqslant T}\|u(t)\|_{E}+\sup _{0 \leqslant t<t+\tau \leqslant T} \frac{\|u(s+\tau)-u(s)\|_{E}}{\tau^{\gamma}} .
$$

Пусть банахово пространство $C_{0}^{\gamma}([0, T] ; E)$ является пополнением пространства гладких функций $u(\cdot)$, заданных на отрезке $[0, T]$ и принимающих значения в $E$, относительно следующей нормы:

$$
\|u(\cdot)\|_{C_{0}^{\gamma}([0, T] ; E)}=\|u(\cdot)\|_{C([0, T] ; E)}+\sup _{0 \leqslant t<t+\tau \leqslant T} t^{\gamma} \frac{\|u(t+\tau)-u(t)\|_{E}}{\tau^{\gamma}} .
$$

Легко доказать, что $C^{\gamma}([0, T] ; E) \subset C_{0}^{\gamma}([0, T] ; E) \subset C([0, T] ; E)$.

Определение 5.5.1. Будем говорить, что задача (5.5.2) корректно поставлена (корректна) в $C_{0}^{\gamma}([0, T] ; E)$, если выполнены следующие условия:

(i) для любой функции $f(\cdot) \in C_{0}^{\gamma}([0, T] ; E)$ и $u^{0} \in D(A)$ существует единственное решение $u(t)=u\left(t ; f, u^{0}\right)$ задачи $(5.5 .2)$ на отрезке $[0, T]$ и $u(\cdot) \in C_{0}^{\gamma}([0, T] ; E)$;

(ii) функция $u(t)=u\left(t ; f, u^{0}\right)$, рассматриваемая как оператор из $C_{0}^{\gamma}([0, T] ; E) \times D(A)$ в $C_{0}^{\gamma}([0, T] ; E)$, непрерывна, где пространство $C_{0}^{\gamma}([0, T] ; E) \times D(A)$ снабжено нормой

$$
\left\|\left(f, u^{0}\right)\right\|_{C_{0}^{\gamma}([0, T] ; E) \times D(A)}=\|f(\cdot)\|_{C_{0}^{\gamma}([0, T] ; E)}+\left\|A u^{0}\right\|_{E} .
$$

Определение 5.5.2. Будем говорить, что задача (5.5.2) коэриитивно корректна (или обладает свойством максимальной регулярности) в пространстве $C_{0}^{\gamma}([0, T] ; E)$, если она корректна в $C_{0}^{\gamma}([0, T] ; E)$ и удовлетворяет следующему неравенству коэрцитивности:

$$
\left\|D^{\alpha} u(\cdot)\right\|_{C_{0}^{\gamma}([0, T] ; E)}+\|A u(\cdot)\|_{C_{0}^{\gamma}([0, T] ; E)} \leqslant C\left(\|f(\cdot)\|_{C_{0}^{\gamma}([0, T] ; E)}+\left\|A u^{0}\right\|_{E}\right) .
$$

Определение 5.5.3. Функция $u(\cdot) \in C([0, T] ; E)$ называется классическим решением задачи Коши (5.5.2), если для любых $t \in[0, T]$ имеем $u(t) \in D(A), A u(\cdot), D^{\alpha} u(\cdot) \in C([0, T] ; E)$ и, кроме того, $u(\cdot)$ удовлетворяет (5.5.2) на $[0, T]$.

Обозначим через $\mathcal{A}^{\alpha}$ множество операторов $A$, генерирующих аналитические $\alpha$-разрешающие семейства аналитического типа.

Теорема 5.5.1 (см. $[184,190])$. Пусть $0<\alpha, \gamma<1$. Предположим, что $A \in \mathcal{A}^{\alpha}, f(\cdot) \in$ $C_{0}^{\gamma}([0, T] ; E)$ и и $\in$ ( $D(A)$. Тогда существует единственное классическое решение задачи (5.5.2), которое определяется формулой

$$
u(t)=S_{\alpha}(t) u^{0}+\left(P_{\alpha} * f\right)(t), \quad t \in[0, T] .
$$

Теорема 5.5.2 (см. $[184,190])$. Пусть $0<\gamma, \alpha<1$. Предположим, что $A \in \mathcal{A}^{\alpha}, u^{0} \in D(A)$ $u f(\cdot) \in C_{0}^{\gamma}([0, T] ; E)$. Тогда классическое решение $u(\cdot)$ уравнения $(5.5 .2)$ лежит в $C_{0}^{\gamma}([0, T] ; E)$ u оператор $u(t)=u\left(t ; f, u^{0}\right)$, действующий из $C_{0}^{\gamma}([0, T] ; E) \times D(A)$ в $C_{0}^{\gamma}([0, T] ; E)$, непреръвен.

Теорема 5.5.3 (см. $[184,190])$. Пусть $0<\gamma, \alpha<1, A \in \mathcal{A}^{\alpha}, f(\cdot) \in C_{0}^{\gamma}([0, T] ; E) u u^{0} \in D(A)$. Предположим, что и(.) -единственное классическое решение уравнения (5.5.2). Тогда Аи(.), $D^{\alpha} u(\cdot) \in C_{0}^{\gamma}([0, T] ; E) u$

$$
\left\|D^{\alpha} u(\cdot)\right\|_{C_{0}^{\gamma}([0, T] ; E)}+\|A u(\cdot)\|_{C_{0}^{\gamma}([0, T] ; E)} \leqslant C\left(\|f(\cdot)\|_{C_{0}^{\gamma}([0, T] ; E)}+\left\|A u^{0}\right\|_{E}\right) .
$$

Замечание 5.5.1. Чтобы доказать коэрцитивность дробной задачи Коши (5.5.2), нужно рассматривать не пространство $C^{\gamma}([0, T] ; E)$, а пространство $C_{0}^{\gamma}([0, T] ; E)$. В [173] рассматривалась регулярность по Гельдеру для $(5.5 .2)$ в пространстве $C^{\gamma}([0, T] ; E)$. Доказано, что $A u, D^{\alpha} u \in$ $C^{\gamma}([0, T] ; E)$ при дополнительном условии $u^{0}=f(0)=0$. В [126] читатель найдет прекрасное изложение вопросов коэрцитивности в пространстве $C^{\gamma}([0, T] ; E)$. 
5.5.2. Разностные схемы для дробных уравнений. Рассмотрим аппроксимацию дробной начальной задачи (5.5.2). Аппроксимация задачи (5.5.2) на общей дискретизационной схеме в банаховом пространстве $E_{n}$ имеет вид

$$
\left\{\begin{array}{l}
D^{\alpha} u_{n}(t)=A_{n} u_{n}(t)+f_{n}(t), \quad 0 \leqslant t \leqslant T \\
u_{n}(0)=u_{n}^{0}
\end{array}\right.
$$

Дискретизацию задачи (5.5.4) можно провести при помощи различных разностных схем. Сначала рассмотрим аппроксимацию задачи (5.5.4) неявной схемой

$$
\Delta_{t_{k}}^{\alpha} \bar{U}_{n}(\cdot)=A_{n} \bar{U}_{n}\left(t_{k}\right)+f_{n}\left(t_{k}\right), \quad \bar{U}_{n}(0)=x_{n} .
$$

Хорошо известно, что (5.5.5) можно разложить следующим образом:

$$
\begin{aligned}
\Delta_{t_{k}}^{\alpha} \bar{W}_{n}(\cdot) & =A_{n} \bar{W}_{n}\left(t_{k}\right), & & \bar{W}_{n}(0)=x_{n}, \\
\Delta_{t_{k}}^{\alpha} \bar{V}_{n}(\cdot) & =A_{n} \bar{V}_{n}\left(t_{k}\right)+f_{n}\left(t_{k}\right), & & \bar{V}_{n}(0)=0 ;
\end{aligned}
$$

таким образом, $\bar{U}_{n}\left(t_{k}\right)=\bar{W}_{n}\left(t_{k}\right)+\bar{V}_{n}\left(t_{k}\right)$. Тогда из [188, предложение 3.1 , теорема 3.2$]$ получаем следующую теорему.

Теорема 5.5.4 (см. [191]). Для неявной разностной схемы (5.5.5), т.е. для системы

$$
\frac{1}{\Gamma(2-\alpha)} \sum_{j=0}^{k-1} b_{j} \frac{\bar{U}_{n}\left((k-j) \tau_{n}\right)-\bar{U}_{n}\left((k-j-1) \tau_{n}\right)}{\tau_{n}^{\alpha}}=A_{n} \bar{U}_{n}\left(k \tau_{n}\right)+f_{n}\left(k \tau_{n}\right), \quad \bar{U}_{n}(0)=x_{n},
$$

имеем

$$
\bar{U}_{n}\left(k \tau_{n}\right)=\sum_{j=1}^{k} c_{j}^{(k)} R^{j} x_{n}+\Gamma(2-\alpha) \tau_{n}^{\alpha} \sum_{j=1}^{k} \sum_{i=1}^{k-j+1} d_{i, j}^{(k)} R^{i} f_{n}\left(j \tau_{n}\right) \quad \text { для любого } k \in \mathbb{N},
$$

где $c_{j}^{(k)} u d_{i, j}^{(k)}$-такие же, как в $[188,189]$. Кроме того, для любого $k \in \mathbb{N}$ имеем $c_{j}^{(k)}>0$, $j=1,2, \ldots, k, d_{i, j}^{(k)} \geqslant 0, i=1, \ldots, k-j+1, j=1, \ldots, k$,

$$
\sum_{j=1}^{k} c_{j}^{(k)}=1, \quad \sum_{j=1}^{k} \sum_{i=1}^{k-j+1} d_{i, j}^{(k)} b_{j-1}=1
$$

Теорема 5.5.5 (см. [191]). Предположим, что $C_{0}$-полугруппы $e^{t A_{n}}$ удовлетворяют условию (В) $и \omega=0$. Тогда неявная разностная схема (5.5.5) устойчива, т.е.

$$
\sup _{1 \leqslant j \leqslant k}\left\|\bar{U}_{n}\left(j \tau_{n}\right)\right\| \leqslant M\left\|x_{n}\right\|+M \Gamma(1-\alpha)\left(k \tau_{n}\right)^{\alpha} \sup _{1 \leqslant j \leqslant k}\left\|f_{n}\left(j \tau_{n}\right)\right\| .
$$

В следующей теореме установлен порядок сходимости неявной разностной схемы (5.5.5). Положим $\bar{z}_{u_{n}}\left(k \tau_{n}\right)=u_{n}\left(k \tau_{n}\right)-\bar{U}_{n}\left(k \tau_{n}\right)$.

Теорема 5.5.6 (см. [191]). Справедливо следующее представление:

$$
\bar{z}_{u_{n}}\left(k \tau_{n}\right)=\Gamma(2-\alpha) \tau_{n}^{\alpha} \sum_{j=1}^{k} \sum_{i=1}^{k-j+1} d_{i, j}^{(k)} R^{i} \bar{r}_{u_{n}}\left(j \tau_{n}\right)
$$

где $d_{i, j}^{(k)}$ такие же, как в $[188,189]$. Кроме того, если $C_{0}$-полугруппь е tAn $_{n}$ удовлетворяют условию (B) $u \omega=0$, mо

$$
\sup _{1 \leqslant j \leqslant k}\left\|\bar{z}_{u_{n}}\left(j \tau_{n}\right)\right\| \leqslant C(\alpha) \tau_{n}^{\alpha} m\left(x_{n}, f_{n}\right), \quad 0 \leqslant k \tau_{n} \leqslant T
$$

где

$$
m\left(x_{n}, f_{n}\right)=\max \left\{\left\|x_{n}\right\|,\left\|A_{n} x_{n}\right\|,\left\|A_{n}^{2} x_{n}\right\|,\left\|f_{n}(\cdot)\right\|_{C^{2}\left([0, T] ; E_{n}\right)}\right\} .
$$


Далее, явную схему

$$
\Delta_{t_{k}}^{\alpha} U_{n}(\cdot)=A_{n} U_{n}\left(t_{k-1}\right)+f_{n}\left(t_{k-1}\right), \quad U_{n}(0)=x_{n},
$$

можно разложить следующим образом:

$$
\begin{aligned}
\Delta_{t_{k}}^{\alpha} W_{n}(\cdot) & =A_{n} W_{n}\left(t_{k}\right), & & W_{n}(0)=x_{n} \\
\Delta_{t_{k}}^{\alpha} V_{n}(\cdot) & =A_{n} V_{n}\left(t_{k}\right)+f_{n}\left(t_{k}\right), & & V_{n}(0)=0,
\end{aligned}
$$

так что $U_{n}\left(t_{k}\right)=W_{n}\left(t_{k}\right)+V_{n}\left(t_{k}\right)$. Имеет место следующая теорема.

Теорема 5.5.7 (см. [191]). Для явной схемы (5.5.9), т.е. для системы

$\frac{1}{\Gamma(2-\alpha)} \sum_{j=0}^{k-1} b_{j} \frac{U_{n}\left((k-j) \tau_{n}\right)-U_{n}\left((k-j-1) \tau_{n}\right)}{\tau_{n}^{\alpha}}=A_{n} U_{n}\left((k-1) \tau_{n}\right)+f_{n}\left((k-1) \tau_{n}\right), \quad U_{n}(0)=x_{n}$, имеем

$$
U_{n}\left(k \tau_{n}\right)=\sum_{j=0}^{k} \bar{c}_{j}^{(k)} \bar{R}^{j} x_{n}+\Gamma(2-\alpha) \tau_{n}^{\alpha} \sum_{j=0}^{k-1} \sum_{i=0}^{k-j-1} \bar{d}_{i, j+1}^{(k+1)} \bar{R}^{i} f_{n}\left(j \tau_{n}\right) \quad \text { для любого } k \in \mathbb{N},
$$

где $\bar{c}_{j}^{(k)}$ и $\bar{d}_{i, j}^{(k)}$-такие же, как в $[188,189]$. Кроме того, для любого $k \in \mathbb{N}$ имеем $\bar{c}_{j}^{(k)}>0$, $j=0,1, \ldots, k, \bar{d}_{i, j+1}^{(k+1)} \geqslant 0, i=0, \ldots, k-j-1, j=0, \ldots, k-1$,

$$
\sum_{j=0}^{k} \bar{c}_{j}^{(k)}=1, \quad \sum_{j=0}^{k-1} \sum_{i=0}^{k-j-1} \bar{d}_{i, j+1}^{(k+1)} b_{j}=1 .
$$

Замечание 5.5.2. В нашем изложении $V_{n}\left(k \tau_{n}\right)$ отличается от аналогичной величины в [188, 189], поскольку $f_{n}(0) \neq 0$, в то время как в $[188,189]$ выполнялось условие $f_{n}(0)=0$.

Теорема 5.5.8 (см. [191]). Пусть $\alpha>1 / 2 u e^{t A_{n}}-C_{0}$-полугруппы, порожденные операторами $A_{n}$. Предположим, что полугруппы $e^{t A_{n}}$ удовлетворяют условию (В) $u \omega=0$. Если

$$
\left\|\tau_{n}^{2 \alpha-1} A_{n}^{2}\right\| \leqslant c
$$

где с не зависит от п, то для схемь (5.5.9) имеем

$$
\sup _{0 \leqslant j \leqslant k}\left\|U_{n}\left(j \tau_{n}\right)\right\| \leqslant \bar{M}\left\|x_{n}\right\|+\bar{M} \Gamma(1-\alpha)\left(k \tau_{n}\right)^{\alpha} \sup _{0 \leqslant j \leqslant k-1}\left\|f_{n}\left(j \tau_{n}\right)\right\| .
$$

Теорема 5.5.9 (см. [191]). Для явной разностной схемы (5.5.9) имеем следующее представление разности $z_{u_{n}}\left(k \tau_{n}\right)=u_{n}\left(k \tau_{n}\right)-U_{n}\left(k \tau_{n}\right)$ :

$$
z_{u_{n}}\left(k \tau_{n}\right)=\Gamma(2-\alpha) \tau_{n}^{\alpha} \sum_{j=1}^{k} \sum_{i=0}^{k-j} \bar{d}_{i, j}^{(k+1)} \bar{R}^{i} r_{u_{n}}\left(j \tau_{n}\right)
$$

где

$$
r_{u_{n}}\left(k \tau_{n}\right)=\Delta_{t_{k}}^{\alpha} u_{n}(\cdot)-\left(\mathbf{D}_{t}^{\alpha} u_{n}\right)\left(t_{k-1}\right),
$$

$\bar{d}_{i, j}^{(k)}$ такое же, как в $[188,189]$. Если $\alpha>1 / 2$, то предположим, что $C_{0}$-полугруппъ $e^{t A_{n}}$ удовлетворяют условию (В) $и \omega=0$. Кроме того, если

$$
\left\|\tau_{n}^{2 \alpha-1} A_{n}^{2}\right\| \leqslant c
$$

где с не зависит от $n$, то

$$
\sup _{1 \leqslant j \leqslant k}\left\|z_{u_{n}}\left(j \tau_{n}\right)\right\| \leqslant C(\alpha) \tau_{n}^{\alpha} m\left(x_{n}, f_{n}\right)
$$


Теорема 5.5.10 (см. [191]). Для $z_{u_{n}}\left(k \tau_{n}\right)=u_{n}\left(k \tau_{n}\right)-U_{n}\left(k \tau_{n}\right)$ имеет место следующее представление:

$$
z_{u_{n}}\left(k \tau_{n}\right)=\Gamma(2-\alpha) \tau_{n}^{\alpha} \sum_{j=1}^{k} \sum_{i=0}^{k-j} \bar{d}_{i, j}^{(k+1)} \bar{R}^{i} r_{u_{n}}\left(j \tau_{n}\right)
$$

где

$$
r_{u_{n}}\left(k \tau_{n}\right)=\Delta_{t_{k}}^{\alpha} u_{n}(\cdot)-\left(\mathbf{D}_{t}^{\alpha} u_{n}\right)\left(t_{k-1}\right),
$$

$\bar{d}_{i, j}^{(k)}$ такое же, как в $[188,189]$. Предположим, что выполнено условие $\left(\mathrm{B}_{1}\right), \omega=0$ и

$$
\left\|\frac{\Gamma(2-\alpha)}{\left(1-b_{1}\right)} \tau_{n}^{\alpha} A_{n}\right\| \leqslant c
$$

где $c<1 /(M+2)$ не зависит от $n$. Тогда

$$
\sup _{1 \leqslant j \leqslant k}\left\|z_{u_{n}}\left(j \tau_{n}\right)\right\| \leqslant C(\alpha) \tau_{n}^{\alpha} m\left(x_{n}, f_{n}\right) .
$$

В [55] обсуждалось соотношение между дробными степенями положительных операторов и дробными производными; в частности, было доказано, что

$$
\Lambda^{\alpha} \vartheta(\xi)=D^{\alpha} \vartheta(\xi)
$$

где $\Lambda \vartheta(\xi)=\vartheta^{\prime}(\xi), D(\Lambda)=\left\{\vartheta(\xi): \vartheta^{\prime}(\xi) \in C[0, T], \vartheta(0)=0\right\}, \Lambda^{\alpha}$ - дробная степень оператора $\Lambda$ и $D_{t_{k}}^{\alpha} \vartheta(\cdot)$ - дробная производная Римана-Лиувилля функции $\vartheta(\cdot) \in D(\Lambda)$. На основе этого введем следующую дробную разностную производную Римана-Лиувилля порядка $\alpha$ как дискретизацию дробной производной Римана-Лиувилля:

$$
\Delta_{t_{k}}^{\alpha} u_{n}(\cdot)=\frac{1}{\Gamma(1-\alpha)} \sum_{j=1}^{k} \frac{\Gamma(k-j-\alpha+1)}{(k-j) !} \frac{u_{n}\left(t_{j}\right)-u_{n}\left(t_{j-1}\right)}{\tau_{n}^{\alpha}} .
$$

Следовательно, можно аппроксимировать задачу (5.5.2) следующей неявной разностной схемой:

$$
\left\{\begin{array}{l}
\Delta_{t_{k}}^{\alpha} \bar{U}_{n}(\cdot)=A_{n} \bar{U}_{n}\left(t_{k}\right)+f_{n}\left(t_{k}\right), \\
\bar{U}_{n}(0)=u_{n}^{0}
\end{array}\right.
$$

где $t_{k}=k \tau_{n} \in[0, T], k=1,2, \ldots, K$.

Введем обозначение

Тогда в терминах (5.5.12) имеем

$$
b_{j}^{(k)}=\frac{\Gamma(k-j-\alpha+1)}{(k-j) ! \cdot \Gamma(1-\alpha)} .
$$

$$
\sum_{j=1}^{k} b_{j}^{(k)} \frac{\bar{U}_{n}\left(t_{j}\right)-\bar{U}_{n}\left(t_{j-1}\right)}{\tau_{n}^{\alpha}}=A_{n} \bar{U}_{n}\left(t_{k}\right)+f_{n}\left(t_{k}\right) .
$$

Обозначим $R=\left(I_{n}-\tau_{n}^{\alpha} A_{n}\right)^{-1}$. Тогда

$$
\bar{U}_{n}\left(t_{k}\right)=R b_{1}^{(k)} \bar{U}_{n}(0)+R \sum_{j=2}^{k}\left(b_{j}^{(k)}-b_{j-1}^{(k)}\right) \bar{U}_{n}\left(t_{j-1}\right)+R \tau_{n}^{\alpha} f_{n}\left(t_{k}\right) .
$$

Замечание 5.5.3. Отметим, что величины $b_{j}^{(k)}$ и $\Delta_{t_{k}}^{\alpha} \bar{U}_{n}(\cdot)$ отличаются от $b_{j}$ и $\Delta_{t_{k}}^{\alpha} \bar{U}_{n}(\cdot)$, введенных в [188], где $b_{j}=(j+1)^{1-\alpha}-j^{1-\alpha}$. Таким образом, неявная разностная схема (5.5.12) отличается от схемы (5.5.5).

Теорема 5.5.11 (см. [184]). Для неявной разностной схемъ (5.5.12) имеет место следующее соотношение:

$$
\bar{U}_{n}\left(k \tau_{n}\right)=\sum_{i=1}^{k} c_{i}^{(k)} R^{i} u_{n}^{0}+\tau_{n}^{\alpha} \sum_{j=1}^{k} \sum_{i=1}^{k-j+1} d_{i, j}^{(k)} R^{i} f_{n}\left(j \tau_{n}\right),
$$


где

$$
\begin{array}{lll}
c_{1}^{(k)}=b_{1}^{(k)}, & c_{i}^{(k)}=\sum_{j=i}^{k}\left(b_{j}^{(k)}-b_{j-1}^{(k)}\right) c_{i-1}^{(j-1)}, & i=2,3, \ldots, k, \\
d_{1, j}^{(k)}=0, & d_{i, j}^{(k)}=\sum_{m=i+j-1}^{k}\left(b_{m}^{(k)}-b_{m-1}^{(k)}\right) d_{i-1, j}^{(m-1)}, & i=2,3, \ldots, k-j+1, \quad d_{1, k}^{(k)}=1 .
\end{array}
$$

Кроме того,

$$
\begin{array}{lll}
c_{i}^{(k)}>0, & i=1,2, \ldots, k, & \\
d_{i=1}^{k} \geqslant 0, & \begin{array}{l}
k=1,2, \ldots, k-j+1, \\
j=1,2, \ldots, k,
\end{array} & \sum_{j=1}^{k} \sum_{i=1}^{k-j+1} d_{i, j}^{(k)}=\frac{(1+\alpha)(2+\alpha) \cdots(k-1+\alpha)}{(k-1) !}, k \geqslant 2 .
\end{array}
$$

Теорема 5.5.12 (см. [184]). Предположим, что выполнено условие (В) $u \omega=0$. Тогда нелвная разностная схема (5.5.12) устойчива, т.е.

$$
\sup _{1 \leqslant j \leqslant k}\left\|\bar{U}_{n}\left(k \tau_{n}\right)\right\| \leqslant M_{1}\left\|u_{n}^{0}\right\|+M_{1} \exp (\alpha)\left(k \tau_{n}\right)^{\alpha} \sup _{1 \leqslant j \leqslant k}\left\|f_{n}\left(j \tau_{n}\right)\right\|
$$

где $k \tau_{n} \in[0, T]$.

\section{ГЛАВА 6}

\section{УСТОЙЧИВЫЕ МНОГООБРАЗИЯ ДЛЯ ДРОБНЫХ ПОЛУЛИНЕЙНЫХ УРАВНЕНИЙ}

Эта глава посвящена численному анализу абстрактных полулинейных дробных задач

$$
\mathcal{D}^{\alpha} u(t)=A u(t)+f(u(t)), u(0)=u^{0},
$$

в банаховом пространстве $E$. Мы опишем общий подход к полудискретной аппроксимации устойчивых многообразий. Фазовое пространство в окрестности гиперболического равновесия расщепляется таким образом, что исходная начальная задача редуцируется к системе начальных задач в инвариантных подпространствах, соответствующих положительным и отрицательным вещественным частям спектра. Покажем, что структура такого разложения уравнения сохраняется и при переходе к общей аппроксимационной схеме. Основные предположения, требуемые для наших результатов, естественным образом выполнены, например, для операторов с компактными резольвентами.

Поведение решений полулинейных задач в окрестности гиперболических точек равновесия классических дифференциальных уравнений хорошо изучено (см., например, [?, 26, 79, 86, 89, 136, $214,273])$, так же, как и различные приложения (см., например, $[159,170,171])$. Ситуация существенно меняется, если вместо классических дифференциальных уравнений рассматриваются дробные уравнения.

Например, в [250] установлены некоторые достаточные условия, обеспечивающие свойство притяжения решений полулинейных дробных дифференциальных уравнений с нелинейностями сверхлинейного роста на конечном интервале времени.

Одним из принципиальных отличий является то, что решения дробных задач сходятся к положениям равновесия только с полиномиальной скоростью, а не с экспоненциальной, как в классическом случае. Имеются работы, посвященные проблеме существования устойчивых многообразий для полулинейных дробных уравнений (см. [94,95,102,235]). В [235] проведена аппроксимация локальных устойчивых многообразий вблизи гиперболических точек равновесия дробных дифференциальных уравнений и исследована сходимость численных схем. Ни в одной из упомянутых 
работ не рассматриваются аппроксимации устойчивых многообразий на общей аппроксимационной схеме.

\section{1. ФОРМУЛИРОВКА ДРОБНЫХ ПОЛУЛИНЕЙНЫХ ЗАДАЧ}

В этом разделе будем изучать полудискретную аппроксимацию задачи Коши

$$
\left(\mathcal{D}^{\alpha} u\right)(t)=A u(t)+f(u(t)), \quad 0 \leqslant t \leqslant \infty, \quad u(0)=u^{0},
$$

где $\mathcal{D}^{\alpha}, 0<\alpha \leqslant 1,-$ производная Капуто-Джрбашяна, оператор $A$ порождает аналитическую и компактную $C_{0}$-полугруппц $\exp (\cdot A)$, а функция $f(\cdot)$ является достаточно гладкой. Точнее, предположим, что $A: D(A) \subseteq E \rightarrow E$ - такой замкнутый линейный оператор, что

$$
\left\|(\lambda I-A)^{-1}\right\|_{B(E)} \leqslant \frac{M}{1+|\lambda|} \text { для любого } \lambda, \operatorname{Re} \lambda \geqslant 0,
$$

и функция $f: E^{\beta} \subseteq E \rightarrow E, 0 \leqslant \beta<1$, ограничена и непрерывно дифференцируема по Фреше.

Поскольку $C_{0}$-полугруппа $\exp (\cdot A)$ существует, то $\alpha$-разрешающее семейство $S_{\alpha}(t, A) \equiv E_{\alpha}\left(t^{\alpha} A\right)$, $t \geqslant 0$, аналитично по $t$ (см. [66, теорема 3.3]), и мы имеем (см. [105, 152]),

$$
E_{\alpha}\left(t^{\alpha} A\right) x=\int_{0}^{\infty} t^{-\alpha} \Phi_{\alpha}\left(\xi t^{-\alpha}\right) \exp (\xi A) x d \xi \quad \text { для любых } x \in E \text { и } t>0,
$$

где $\Phi_{\alpha}(\cdot)$ функция типа Райта (см. [66]):

$$
\Phi_{\alpha}(0)=\frac{1}{\Gamma(1-\alpha)}, \quad \Phi_{\alpha}(t) \geqslant 0, \quad t>0, \quad \int_{0}^{\infty} \Phi_{\alpha}(t) d t=1 .
$$

Решение задачи (6.1.1) в неоднородном случае задается формулой

$$
u(t)=E_{\alpha}\left(t^{\alpha} A\right) u^{0}+\int_{0}^{t} P_{\alpha}(t-s, A) f(u(s)) d s, \quad t \geqslant 0,
$$

где

$$
P_{\alpha}(t, A) x=\alpha \int_{0}^{\infty} \frac{s}{t^{\alpha+1}} \Phi_{\alpha}\left(s t^{-\alpha}\right) \exp (s A) x d s=\alpha \int_{0}^{\infty} \frac{\tau}{t^{1-\alpha}} \Phi_{\alpha}(\tau) \exp \left(\tau t^{\alpha} A\right) x d \tau
$$

для любого $x \in E$ и $P_{\alpha}(t, A)$ аналитично по $t$ для любого $t>0$ (см. [152]). Кроме того, (5.1.4) и (5.3.2) выполняются для любого $\operatorname{Re} \lambda>\omega, x \in E$. Отметим, что в скалярном случае, т.е. если $A=\gamma I$, имеем $S_{\alpha}(t, \gamma)=E_{\alpha}\left(\gamma t^{\alpha}\right)$, где $E_{\alpha}(\cdot)$ - функция Миттаг-Леффлера

$$
E_{\alpha, \beta}(z)=\sum_{n=0}^{\infty} \frac{z^{n}}{\Gamma(\alpha n+\beta)}, \quad E_{\alpha}(z)=E_{\alpha, 1}(z) .
$$

Определение 6.1.1. Функция $u(\cdot) \in C([0, \infty) ; E)$ называется обобщенным решением задачи (6.1.1), если она удовлетворяет уравнению (6.1.4).

Равновесные решения задачи (6.1.1) - это решения уравнения

$$
A u+f(u)=0 .
$$

Пусть $\tilde{\omega}>0$ и

$$
\Lambda_{\alpha}^{+}=\tilde{\omega}+\left\{\lambda \in \mathbb{C}:|\arg (\lambda)|<\frac{\alpha \pi}{2}\right\} .
$$

В случае, когда резольвента $(\lambda I-A)^{-1}$ компактна, часть $\sigma^{+}$спектра оператора $A+f^{\prime}\left(u^{*}\right)$, расположенная строго справа от мнимой оси, состоит из конечного числа собственных значений конечной кратности. 
Определение 6.1.2. Будем называть решение $u^{*}$ задачи (6.1.6) гиперболическим, если спектр $\sigma\left(A+f^{\prime}\left(u^{*}\right)\right)$ не имеет общих точек с мнимой осью т.е.

$$
\sigma\left(A+f^{\prime}\left(u^{*}\right)\right) \cap\{\lambda \in \mathbb{C}:-\varepsilon \leqslant \operatorname{Re} \lambda \leqslant \varepsilon\}=\varnothing
$$

для некоторого $\varepsilon>0$ и $\sigma^{+} \subset \Lambda_{\alpha}^{+}$.

\section{2. СУЩЕСТВОВАНИЕ УСТОЙЧИВЫХ МНОГООБРАЗИЙ}

В этом разделе предположим, что условие (F1) выполнено для гиперболической точки равновесия задачи (6.1.1).

Используя замену переменных $v(\cdot)=u(\cdot)-u^{*}$ в задаче $(6.1 .1)$, где $u^{*}$-гиперболическое равновесие, получим задачу

$$
\begin{aligned}
& \left(\mathcal{D}^{\alpha} v\right)(t)=\left(A+f^{\prime}\left(u^{*}\right)\right) v(t)+f\left(v(t)+u^{*}\right)-f\left(u^{*}\right)-f^{\prime}\left(u^{*}\right) v(t), \\
& v(0)=u^{0}-u^{*}=v^{0}
\end{aligned}
$$

которую можно переписать в виде

$$
\left(\mathcal{D}^{\alpha} v\right)(t)=A_{u^{*}} v(t)+F_{u^{*}}(v(t)), \quad v(0)=v^{0}, \quad t \geqslant 0,
$$

где $A_{u^{*}}=A+f^{\prime}\left(u^{*}\right), F_{u^{*}}(v(t))=f\left(v(t)+u^{*}\right)-f\left(u^{*}\right)-f^{\prime}\left(u^{*}\right) v(t)$. Заметим, что

$$
F_{u^{*}}(v(t))=f\left(v(t)+u^{*}\right)-f\left(u^{*}\right)-f^{\prime}\left(u^{*}\right) v(t)=o\left(\|v(t)\|_{E^{\beta}}\right) \quad \text { для малых }\left\|v^{0}\right\|_{E^{\beta}} .
$$

Поскольку $f^{\prime}\left(u^{*}\right) \in B\left(E^{\beta}, E\right), 0 \leqslant \beta<1$, оператор $A_{u^{*}}=A+f^{\prime}\left(u^{*}\right)$ является генератором аналитической $C_{0}$-полугруппы (см. [26]).

В случае гиперболической точки равновесия $u^{*}$ на мнимой оси $i \mathbb{R}$ нет точек спектра оператора $A_{u^{*}}$. Пусть $U\left(\sigma^{+}\right) \subset\{\lambda \in \mathbb{C}: \operatorname{Re} \lambda>0\}$ - открытая связная окрестность множества $\sigma^{+}$, границей которой является замкнутая спрямляемая кривая $\partial U\left(\sigma^{+}\right)$. Разложим $E^{\beta}$ при помощи проекторов Рисса

$$
P\left(\sigma^{+}\right)=P\left(\sigma^{+}, A_{u^{*}}\right)=\frac{1}{2 \pi i} \int_{\partial U\left(\sigma^{+}\right)}\left(\zeta I-A_{u^{*}}\right)^{-1} d \zeta
$$

определенных множеством $\sigma^{+}$. В силу определения и аналитичности $C_{0}$-полугрупп $e^{t A_{u^{*}}}, t \in \overline{\mathbb{R}}^{+}$, найдутся такие положительные константы $M$ и $\omega$, что неравенства (3.1.13) выполняются при $\theta=\beta$. Без ограничения общности можно выбрать в $E^{\beta}$ такую норму, что

$$
\|v\|_{E^{\beta}}=\max \left(\|P(\sigma+) v\|_{E^{\beta}},\|(I-P(\sigma+)) v\|_{E^{\beta}}\right) .
$$

Ясно, что эта норма эквивалентна исходной норме в $E^{\beta}$.

Нам понадобятся следующие факты (см. [155, 223]).

Лемма 6.2.1. Пусть $0<\alpha<1 u \omega>0$. Тогда справедливы следующие утверждения:
(i) $E_{\alpha}\left(\omega t^{\alpha}\right)=\frac{1}{\alpha} e^{\omega^{1 / \alpha} t}+O\left(\frac{1}{t^{\alpha}}\right)$
npu $t \rightarrow \infty$
(ii) $E_{\alpha}\left(\omega t^{\alpha}\right) \rightarrow 1$
npu $t \rightarrow 0$;
(iii) $t^{\alpha-1} E_{\alpha, \alpha}\left(\omega t^{\alpha}\right)=\frac{1}{\alpha} \omega^{1 / \alpha-1} e^{\omega^{1 / \alpha} t}+O\left(\frac{1}{t^{\alpha+1}}\right)$
npu $t \rightarrow \infty$
(iv) $E_{\alpha, \alpha}\left(\omega t^{\alpha}\right) \rightarrow \frac{1}{\Gamma(\alpha)}$
npu $t \rightarrow 0$. 
Лемма 6.2.2. Пусть $0<\alpha<1 u \omega>0$. Тогда справедливы следующие утвержсения:

$$
\begin{array}{lll}
\text { (i) } \quad E_{\alpha}\left(-\omega t^{\alpha}\right)=O\left(\frac{1}{t^{\alpha}}\right) & \text { npu } t \rightarrow \infty ; \\
\text { (ii) } \quad E_{\alpha}\left(-\omega t^{\alpha}\right) \rightarrow 1 & \text { npu } t \rightarrow 0 ; \\
\text { (iii) } \quad t^{\alpha-1} E_{\alpha, \alpha}\left(-\omega t^{\alpha}\right)=O\left(\frac{1}{t^{\alpha+1}}\right) & \text { npu } t \rightarrow \infty ; \\
\text { (iv) } \quad E_{\alpha, \alpha}\left(-\omega t^{\alpha}\right) \rightarrow \frac{1}{\Gamma(\alpha)} & \text { npu } t \rightarrow 0 .
\end{array}
$$

Лемма 6.2.3. Пусть $\exp (t A), t \geqslant 0,-$ аналитическая $C_{0}$-полугруппа, удовлетворяющая оиенке

$$
\|\exp (t A)\| \leqslant M e^{-\omega t}
$$

nрu $\omega>0, t \geqslant 0 u 0<\alpha, \beta<1$. Тогда

$$
\begin{array}{ll}
\left\|(-A)^{\beta} P_{\alpha}(t, A) x\right\| \leqslant \frac{C}{t^{1-\alpha(1-\beta)}}\|x\| & \text { для всех } x \in E, t \rightarrow 0, \\
\left\|(-A)^{\beta} P_{\alpha}(t, A) x\right\| \leqslant \frac{C_{1}}{t^{1+\alpha}}\|x\| & \text { для всех } x \in E, t \rightarrow \infty .
\end{array}
$$

Доказательство. Из (6.1.5) находим

$$
\begin{aligned}
\left\|(-A)^{\beta} P_{\alpha}(t, A) x\right\| \leqslant \alpha & \int_{0}^{\infty} \frac{s}{t^{\alpha+1}} \Phi_{\alpha}\left(s t^{-\alpha}\right) \frac{M}{s^{\beta}} e^{-\omega s}\|x\| d s \leqslant \\
& \leqslant M \int_{0}^{\infty} \frac{\left(\xi t^{\alpha}\right)^{1-\beta}}{t^{\alpha+1}} \Phi_{\alpha}(\xi) e^{-\omega \xi t^{\alpha}} t^{\alpha} d \xi\|x\| \leqslant C \int_{0}^{\infty} \frac{\xi^{1-\beta}}{t^{1-\alpha(1-\beta)}} \Phi_{\alpha}(\xi) e^{-\omega \xi t^{\alpha}} d \xi\|x\| .
\end{aligned}
$$

Поскольку

$$
\left|e^{-\omega \xi t^{\alpha}}\right| \leqslant 1, \quad \int_{0}^{\infty} \xi^{1-\beta} \Phi_{\alpha}(\xi) d \xi=\frac{\Gamma(2-\beta)}{\Gamma(1+\alpha(1-\beta))}
$$

получаем оценку (6.2.3). Чтобы получить (6.2.4), воспользуемся (6.1.5):

$$
\int_{0}^{\infty} \frac{\left(\xi t^{\alpha}\right)^{1-\beta}}{t^{\alpha+1}} \Phi_{\alpha}(\xi) e^{-\omega \xi t^{\alpha}} t^{\alpha} d \xi\|x\| \leqslant C \int_{0}^{\infty} \frac{\left(\omega \xi t^{\alpha}\right)^{1-\beta}}{t^{1+\alpha}} \Phi_{\alpha}(\xi) e^{-\omega \xi t^{\alpha}} d\left(\omega \xi t^{\alpha}\right)\|x\| \leqslant \frac{C_{1}}{t^{1+\alpha}}\|x\| .
$$

Теперь разложим уравнение (6.2.1) следующим образом:

$$
w(t)=P\left(\sigma^{+}\right) v(t), \quad z(t)=\left(I-P\left(\sigma^{+}\right)\right) v(t) .
$$

Таким образом, если $A_{u^{*}}^{+}$- сужение оператора $\left(A+f^{\prime}\left(u^{*}\right)\right)$ на $P\left(\sigma^{+}\right) E^{\beta}$ и $A_{u^{*}}^{-}$- сужение оператора $\left(A+f^{\prime}\left(u^{*}\right)\right)$ на $\left(I-P\left(\sigma^{+}\right)\right) E^{\beta}$, то

$$
\begin{aligned}
& \left(\mathcal{D}^{\alpha} w\right)(t)=A_{u^{*}}^{+} w(t)+H(w(t), z(t)), \\
& \left(\mathcal{D}^{\alpha} z\right)(t)=A_{u^{*}}^{-} z(t)+G(w(t), z(t)),
\end{aligned}
$$

где

$$
\begin{aligned}
& H(w, z)=P\left(\sigma^{+}\right) f\left(w(t)+z(t)+u^{*}\right)-P\left(\sigma^{+}\right) f\left(u^{*}\right)-P\left(\sigma^{+}\right) f^{\prime}\left(u^{*}\right)(w(t)+z(t)) \\
& G(w, z)=\left(I-P\left(\sigma^{+}\right)\right) f\left(w(t)+z(t)+u^{*}\right)-\left(I-P\left(\sigma^{+}\right)\right) f\left(u^{*}\right)-\left(I-P\left(\sigma^{+}\right)\right) f^{\prime}\left(u^{*}\right)(w(t)+z(t)) .
\end{aligned}
$$

Таким образом, в точке $(0,0)$ функции $H$ и $G$ и их производные обращаются в нуль. 
Аналогично теореме 3.1.2 находим, что обобщенным решением задачи (6.2.5)-(6.2.6), стремящимся к нулю при $t \rightarrow \infty$, является вектор-функция $v(t)=\left(\begin{array}{c}w(t) \\ z(t)\end{array}\right)$, удовлетворяющая формаль-
ному уравнению

$$
v(t)=\Theta(\eta, v(\cdot))(t),
$$

но с иной структурой $\Theta$ по сравнению с теоремой 3.1.2. Рассмотрим (6.2.7) в пространстве

$$
\begin{aligned}
\Upsilon=C_{\gamma}([0, \infty), P & \left.\left(\sigma^{+}\right) E^{\beta} \times\left(I-P\left(\sigma^{+}\right)\right) E^{\beta}\right)= \\
& =\left\{v(\cdot) \in C\left([0, \infty), P\left(\sigma^{+}\right) E^{\beta} \times\left(I-P\left(\sigma^{+}\right)\right) E^{\beta}\right): v(\cdot) \text { is bounded on }[0, \infty)\right\},
\end{aligned}
$$

снабженном супремум-нормой

$$
\|v\|_{C_{\gamma}}=\|v\|_{\Upsilon}=\sup _{t \geqslant 0} \frac{\|v(t)\|_{E^{\beta}}}{E_{\alpha}\left(-\gamma t^{\alpha}\right)}, \quad \gamma>0 .
$$

Покажем, что $\Theta(\eta, \cdot): \Upsilon \rightarrow \Upsilon$. С этой целью выберем функцию $q(\cdot) \in \Upsilon$, убывающую к нулю как $O\left(t^{-\alpha}\right)$ при $t \rightarrow \infty$. Ясно, что функция $\Theta(\eta, q(\cdot))$ непрерывна по $t$. Покажем, что $\lim _{t \rightarrow \infty} \Theta(\eta, q(\cdot))(t)=0$ и порядок сходимости при $t \rightarrow \infty$ равен $-\alpha$. Любой проектор $P_{j}$ конечномерен, т.е. корневое подпространство, соответствующее собственному значению $\lambda_{j}$, имеем конечную размерность, равную $\operatorname{rank} P_{j}=n_{j}$ и, следовательно, $j$-е уравнение $(j=1, \ldots, K)$ содержит оператор $A_{u^{*}} P_{j}$, который имеет либо вид $\lambda_{j} I_{n_{j} \times n_{j}}$ в случае, если $\lambda_{j}$ - простое собственное значение, либо вид $\lambda_{j} I_{n_{j} \times n_{j}}+N_{n_{j} \times n_{j}}$, где $N_{n_{j} \times n_{j}} \in \mathbb{R}^{n_{j} \times n_{j}}$ - нильпотентная матрица, удовлетворяющая условию $\left(N_{n_{j} \times n_{j}}\right)^{n_{j}}=0$. Изучим эти случаи отдельно.

Сначала рассмотрим случай простого собственного значения $\lambda_{j}$, т.е. $A_{u^{*}} P_{j}=\lambda_{j} I_{n_{j} \times n_{j}}$. Для любых заданных $\varepsilon>0$ и $q(\cdot)$ существует такое $t_{\varepsilon} \in(1, \infty)$, что $\varepsilon \geqslant 1 / t_{\varepsilon}^{\alpha}$ и

$$
\|G(q(s))\|_{\left(I-P\left(\sigma^{+}\right)\right) E^{\beta}}=O\left(s^{-\alpha}\right) \quad \text { для всех } s \geqslant t_{\varepsilon} .
$$

Как было показано в [66, следствие 3.2] и [180], имеем

$$
\left\|E_{\alpha}\left(t^{\alpha} A_{u^{*}}^{-}\right)\right\| \leqslant M E_{\alpha}\left(-\omega t^{\alpha}\right)=O\left(t^{-\alpha}\right) \quad \text { при } t \rightarrow \infty,
$$

так что

$$
\lim _{t \rightarrow \infty} E_{\alpha}\left(t^{\alpha} A_{u^{*}}^{-}\right) \eta=0
$$

с порядком $-\alpha$ при $t \rightarrow \infty$ для любого $\eta \in\left(I-P\left(\sigma^{+}\right)\right) E^{\beta}$. При $t \geqslant t_{\varepsilon}$ из леммы 6.2.3 имеем

$$
\begin{aligned}
& \left\|\left(-A_{u^{*}}\right)^{\beta} \int_{0}^{t} P_{\alpha}\left(t-s, A_{u^{*}}^{-}\right) G(q(s)) d s\right\| \leqslant \\
& \leqslant C\left\|\int_{0}^{t_{\varepsilon}}\left(-A_{u^{*}}\right)^{\beta} P_{\alpha}\left(s, A_{u^{*}}^{-}\right) G(q(t-s)) d s+\int_{t_{\varepsilon}}^{t}\left(-A_{u^{*}}\right)^{\beta} P_{\alpha}\left(s, A_{u^{*}}^{-}\right) G(q(t-s)) d s\right\| \leqslant \\
& \leqslant C \int_{0}^{1} \frac{1}{s^{1-\alpha(1-\beta)}}\|G(q(t-s))\| d s+C \int_{1}^{t_{\varepsilon}} \frac{1}{s^{1+\alpha}}\|G(q(t-s))\| d s+C \int_{t_{\varepsilon}}^{t} \frac{1}{s^{1+\alpha}}\|G(q(t-s))\| d s \leqslant \\
& \leqslant C\left(\varepsilon+\frac{1}{t^{\alpha}}\right) .
\end{aligned}
$$

Таким образом,

$$
\left\|\int_{0}^{t}\left(-A_{u^{*}}\right)^{\beta} P_{\alpha}\left(t-s, A_{u^{*}}^{-}\right) G(q(s)) d s\right\| \leqslant C \varepsilon+O\left(t^{-\alpha}\right) \quad \text { при } t \rightarrow \infty .
$$

для заданного $\varepsilon>0$ 
Из леммы 6.2.1(i) ясно, что

$$
E_{\alpha}\left(t^{\alpha} \lambda_{j}\right) \lambda_{j}^{1 / \alpha-1} \int_{t}^{\infty} \exp \left(-\lambda_{j}^{1 / \alpha} \tau\right)\left\|P_{j} H(w(\tau), z(\tau))\right\| d \tau=O\left(\frac{1}{t^{\alpha}}\right) \quad \text { при } t \rightarrow \infty
$$

поскольку $\left\|P_{j} H(w(\tau), z(\tau))\right\| \leqslant C \tau^{-\alpha}$.

Чтобы оценить первое слагаемое, для произвольного $\varepsilon>0$ найдем такое $t_{\varepsilon}>0$, что $\left\|P_{j} H(w(\tau), z(\tau))\right\| \leqslant \varepsilon$ при $\tau>t_{\varepsilon}$.

Получим, что производная Фреше $\Theta_{q}^{\prime}\left(\eta_{0}, q_{0}\right): \Upsilon \rightarrow \Upsilon$ задается формулой

$$
\left(\Theta_{q}^{\prime}\left(\eta_{0}, q_{0}\right) h\right)(t)=0
$$

для любого $h=\left(\begin{array}{l}h_{1}(t) \\ h_{2}(t)\end{array}\right) \in \Upsilon$, поскольку функции $G$ и $H$ имеют нулевые производные в нуле. Поэтому оператор $\left(I-\Theta_{q}^{\prime}\left(\eta_{0}, q_{0}\right)\right)^{-1}$ существует и непрерывен. Из [21, теорема 54.2] следует, что уравнение (6.2.7) имеет единственное решение.

Рассмотрим случай, в котором $A_{u^{*}} P_{j}=\lambda_{j} I_{n_{j} \times n_{j}}+N_{n_{j} \times n_{j}}$, где собственное значение $\lambda_{j}$ не является простым. Следуя [95], для заданного малого $\delta>0$ применим линейное обратимое преобразование, переводящее $A_{u^{*}} P_{j}$ в $\lambda_{j} I_{n_{j} \times n_{j}}+\delta N_{n_{j} \times n_{j}}$.

Приведем набросок доказательства следующей теоремы.

Теорема 6.2.1 (см. [222]). Пусть и* - гиперболическое равновесие задачи (6.1.1). Предположим, что оператор $A$ порождает аналитическую $C_{0}$-полугруппу $\exp (\cdot A)$ и выполнено условие (F1). Тогда для некоторого достаточно малого $\delta>0$ существует такая липшицнепрерывная функиия

$$
£:\left\{\eta \in\left(I-P\left(\sigma^{+}\right)\right) E^{\beta}:\|\eta\|_{E^{\beta}} \leqslant \delta\right\} \rightarrow P\left(\sigma^{+}\right) E^{\beta}, \quad £(\eta)=w(0),
$$

что локальное устойчивое многообразие $W_{\delta}^{\text {st }}\left(u^{*}\right)$ задается следующим образом:

$$
W_{\delta}^{s t}\left(u^{*}\right)=\left\{(\eta, £(\eta))+u^{*}: \eta \in\left(I-P\left(\sigma^{+}\right)\right) E^{\beta},\|\eta\|_{E^{\beta}} \leqslant \delta\right\} .
$$

\section{3. АППРОКСИМАЦИЯ ДРОБНЫХ РАЗРЕШАЮЩИХ СЕМЕЙСТВ}

В этом разделе мы опишем дискретизацию полулинейных дробных уравнений в соответствии с общей аппроксимационной схемой и обсудим аппроксимацию устойчивых многообразий.

Теорема 6.3.1 (см. $[1,109,185,187,195,271])$. Пусть оператор А является генератором аналитической $C_{0}$-полугруппы $\exp (t A)$ в банаховом пространстве $E$, удовлетворяющей условию $\|\exp (t A)\| \leqslant M e^{-\omega t}, t \geqslant 0$. Тогда компактность резольвенты $R\left(\lambda^{\alpha} ; A\right)$ для некоторого $\lambda^{\alpha} \in \rho(A)$ эквивалентна компактности $S_{\alpha}(t, A)$ для любого $t>0$. Кроме того, компактность $R\left(\lambda^{\alpha} ; A\right)$ для некоторого $\lambda^{\alpha} \in \rho(A)$ эквивалентна компактности $P_{\alpha}(t, A)$ для любого $t>0$.

Доказательство. Докажем только второе утверждение. Известно, что функция $P_{\alpha}(\cdot, A)$ аналитична и компактна. Тогда в силу представления

$$
R\left(\lambda^{\alpha} ; A\right)=\int_{0}^{\infty} e^{-\lambda t} P_{\alpha}(t, A) d t
$$

заключаем, что оператор-функция

$$
R_{q, Q}(\lambda)=\int_{q}^{Q} e^{-\lambda t} P_{\alpha}(t, A) d t
$$

аппроксимирует резольвенту:

$$
\left\|R\left(\lambda^{\alpha} ; A\right)-R_{q, Q}(\lambda)\right\| \rightarrow 0 \quad \text { при } q \rightarrow 0 \text { и } Q \rightarrow \infty .
$$


Оператор-функция $R_{q, Q}(\lambda)$ компактна (см. [264]). Таким образом, резольвента $R\left(\lambda^{\alpha} ; A\right)$ компактна как равномерный предел компактных операторов. Обратно, можем записать (см. [66])

$$
P_{\alpha}(t, A)=\frac{1}{2 \pi i} \int_{\Gamma} e^{\lambda t} R\left(\lambda^{\alpha} ; A\right) d \lambda \quad \text { для любого } t>0,
$$

где $\Gamma$ - положительно ориентированный контур, являющийся границей множества $\omega+\Sigma_{\beta+\pi / 2}$. Поскольку резольвента $R\left(\lambda^{\alpha} ; A\right)$ компактна для любого $\lambda^{\alpha} \in \rho(A)$, получаем, как и выше, что $P_{\alpha}(t, A)$ компактно для любого $t>0$.

Теорема 6.3.2 (см. [187]). Предположим, что операторы $A_{n}$ и А порождают аналитические $C_{0}$-полугруппы, и выполнены условия (A) $и\left(\mathrm{~B}_{1}\right)$ при $\omega_{2}<0$ и резолъвенты $R\left(\lambda^{\alpha} ; A_{n}\right)$ u $R\left(\lambda^{\alpha} ; A\right)$ компактны. Тогда следующие условия эквивалентны:

(i) $R\left(\lambda^{\alpha} ; A_{n}\right) \stackrel{\mathcal{P P}}{\longrightarrow} R\left(\lambda^{\alpha} ; A\right)$ компактно для некоторого $\lambda^{\alpha} \in \rho(A) \cap \bigcap_{n} \rho\left(A_{n}\right)$;

(ii) $S_{\alpha}\left(t, A_{n}\right) \stackrel{\mathcal{P P}}{\longrightarrow} S_{\alpha}(t, A)$ компактно для любого $t>0$;

(iii) $P_{\alpha}\left(t, A_{n}\right) \stackrel{\mathcal{P P}}{\longrightarrow} P_{\alpha}(t, A)$ компактно для любого $t>0$.

Доказательство. Эквивалентность (i) $\Leftrightarrow($ ii) доказана в [187]. Чтобы получить импликацию $(\mathrm{i}) \Leftarrow(\mathrm{iii})$, нужно показать, что мера некомпактности $\mu\left(\left\{R\left(\lambda^{\alpha} ; A_{n}\right) x_{n}\right\}\right)$ равна нулю для любой последовательности $\left\{x_{n}\right\},\left\|x_{n}\right\|_{E_{n}}=O(1)$. Имеем

$$
\begin{array}{r}
\mu\left(\left\{R\left(\lambda^{\alpha} ; A_{n}\right) x_{n}\right\}\right)=\mu\left(\left\{\int_{0}^{\infty} e^{-\lambda t} P_{\alpha}\left(t, A_{n}\right) x_{n} d t\right\}\right) \leqslant \mu\left(\left\{\int_{0}^{q} e^{-\lambda t} P_{\alpha}\left(t, A_{n}\right) x_{n} d t\right\}\right)+ \\
\quad+\mu\left(\left\{\int_{Q}^{\infty} e^{-\lambda t} P_{\alpha}\left(t, A_{n}\right) x_{n} d t\right\}\right)+\mu\left(\left\{\int_{q}^{Q} e^{-\lambda t} P_{\alpha}\left(t, A_{n}\right) x_{n} d t\right\}\right),
\end{array}
$$

где $q$ мало, а $Q$ достаточно велико, так что первое и второе слагаемые меньше произвольно заданного $\varepsilon>0$. Известно, что $\left\{P_{\alpha}\left(t, A_{n}\right)\right\}$ равностепенно непрерывно на $[q, Q]$. Тогда третье слагаемое равно нулю. Импликация (i) $\Rightarrow$ (iii) вытекает из компактной сходимости $\exp \left(t A_{n}\right) \stackrel{\mathcal{P} \mathcal{P}}{\longrightarrow} \exp (t A)$, $t>0$, и представления (6.1.5).

Так же, как для (4.2.4), определим

$$
p_{n}^{\beta}=\left(-A_{n}\right)^{-\beta} p_{n}(-A)^{\beta} \in B\left(E^{\beta}, E_{n}^{\beta}\right) .
$$

Предположим, что операторы $A_{n}$ и $A$ удовлетворяют условиям (6.1.2), $(\mathrm{A})$ и $\left(\mathrm{B}_{1}\right)$.

Рассмотрим в банаховых пространствах $E_{n}^{\beta}, 0 \leqslant \beta<1$, семейство дробных задач

$$
\begin{aligned}
& \left(\mathcal{D}^{\alpha} u_{n}\right)(t)=A_{n} u_{n}(t)+f_{n}\left(u_{n}(t)\right), \quad t \geqslant 0, \\
& u_{n}(0)=u_{n}^{0} \in E_{n}^{\beta},
\end{aligned}
$$

где $u_{n}^{0} \stackrel{\mathcal{P}^{\beta}}{\longrightarrow} u^{0}$, операторы $\left(A_{n}, A\right)$ согласованы, а функции $f_{n}(\cdot): E_{n}^{\beta} \rightarrow E_{n}$ глобально ограничены, глобально липшиц-непрерывны равномерно по $n \in \mathbb{N}$ и непрерывно дифференцируемы по Фреше. Предположим, что операторы $A_{n}$ порождают аналитические $C_{0}$-полугруппы. Предположим также, что $f_{n}\left(x_{n}\right) \stackrel{\mathcal{P}}{\longrightarrow} f(x)$ и $f_{n}^{\prime}\left(x_{n}\right) \stackrel{\mathcal{P}^{\beta} \mathcal{P}}{\longrightarrow} f^{\prime}(x)$ при $x_{n} \stackrel{\mathcal{P}^{\beta}}{\longrightarrow} x$.

При сделанных предположениях обобщенн'мягк'ое решение $u_{n}(\cdot)$ задачи (6.3.1) определено для всех $t \geqslant 0$ и выражается формулой

$$
u_{n}(t)=S_{\alpha}\left(t, A_{n}\right) u_{n}^{0}+\int_{0}^{t} P_{\alpha}\left(t-s, A_{n}\right) f_{n}\left(u_{n}(s)\right) d s, \quad t \geqslant 0 .
$$


В случае $\Delta_{c c} \neq \varnothing$ сходимость $A_{n}^{-1} f_{n}(\cdot) \stackrel{\mathcal{P P}}{\longrightarrow} A^{-1} f(\cdot)$ компактна. Из результатов [259] следует, что при $n \geqslant n_{0}$,

$$
1=\gamma\left(I+A^{-1} f^{\prime}\left(u^{*}\right) ; \partial \Omega\right)=\gamma\left(I_{n}+A_{n}^{-1} f_{n}\left(u_{n}^{*}\right) ; \partial \Omega_{n}\right)
$$

для любого $u^{*} \in E, n \geqslant n_{0}$, и тогда уравнения $u_{n}^{*}=-A_{n}^{-1} f_{n}\left(u_{n}^{*}\right)$ имеют такие решения, что $u_{n}^{*} \stackrel{\mathcal{P}}{\longrightarrow} u^{*}$. Ясно, что $u_{n}^{*} \stackrel{\mathcal{P}^{\beta}}{\longrightarrow} u^{*}$.

Рассмотрим гиперболическое равновесие $u^{*}$ задачи (6.1.1) и гиперболические равновесия $u_{n}^{*} \stackrel{\mathcal{P}^{\beta}}{\longrightarrow} u^{*}$ задач (6.3.1). Кроме того, предположим, что в случае, когда $\theta=\beta$ и $u_{n}^{*}$-гиперболические точки задачи (6.3.1), выполнено условие (F3).

Замечание 6.3.1. Получаем

$$
\left\|F_{u_{n}^{*}, n}^{\prime}\left(q_{n}\right)\right\| \leqslant c_{\rho}\left\|q_{n}\right\|_{E_{n}^{\beta}},
$$

где $c_{\rho} \rightarrow 0$ при $\rho \rightarrow 0$. Здесь $\rho>0$ - радиус шаров $\mathcal{U}_{E_{n}^{\beta}}(0 ; \rho)$, для которых существует такое $\delta>0$, что

$$
\sup _{n \in \mathbb{N}} \sup _{\left\|q_{n}\right\|_{E_{n}^{\beta}} \leqslant \delta}\left\|f_{n}^{\prime}\left(q_{n}+p_{n}^{\beta} u^{*}\right)-f_{n}^{\prime}\left(p_{n}^{\beta} u^{*}\right)\right\|_{B\left(E_{n}^{\beta}, E_{n}\right)} \leqslant \rho .
$$

Теперь рассмотрим семейство дробных задач (6.3.1) в окрестности гиперболической точки равновесия $u_{n}^{*}$. В этом случае имеем

$$
\left(\mathcal{D}^{\alpha} v_{n}\right)(t)=A_{u_{n}^{*}, n} v_{n}(t)+F_{u_{n}^{*}, n}\left(v_{n}(t)\right), \quad v_{n}(0)=v_{n}^{0}, \quad t \geqslant 0
$$

где

$$
A_{u_{n}^{*}, n}=A_{n}+f_{n}^{\prime}\left(u_{n}^{*}\right), \quad F_{u_{n}^{*}, n}\left(v_{n}(t)\right)=f_{n}\left(v_{n}(t)+u_{n}^{*}\right)-f_{n}\left(u_{n}^{*}\right)-f_{n}^{\prime}\left(u_{n}^{*}\right) v_{n}(t) .
$$

Разложим $E_{n}^{\beta}$ при помощи проекционных операторов $P_{n}\left(\sigma_{n}^{+}\right)$так же, как в (4.2.13). Аналогично оператору $\Theta(\eta, u(\cdot))$ (см. (6.2.7)) введем операторы $\Theta_{n}\left(\eta_{n}, u_{n}(\cdot)\right)$. Предположим, что $\eta_{n} \stackrel{\mathcal{P}^{\beta}}{\longrightarrow} \eta$ или даже $\eta_{n}=p_{n}^{\beta} \eta$. Переписав (6.3.1) для функции $v_{n}(t)=u_{n}(t)-u_{n}^{*}$, чтобы иметь возможность работать в окрестности точки $u_{n}^{*}$, приходим к задаче (6.3.3), которую можно переписать в следующем виде:

$$
\left\{\begin{array}{l}
D^{\alpha} w_{n}(t)=\left(A_{n}+f_{n}^{\prime}\left(u_{n}^{*}\right)\right) P_{n}\left(\sigma_{n}^{+}\right) w_{n}(t)+H_{n}\left(w_{n}(t), z_{n}(t)\right), \\
D^{\alpha} z_{n}(t)=\left(A_{n}+f_{n}^{\prime}\left(u_{n}^{*}\right)\right)\left(I_{n}-P_{n}\left(\sigma_{n}^{+}\right)\right) z_{n}(t)+G_{n}\left(w_{n}(t), z_{n}(t)\right),
\end{array}\right.
$$

где $w_{n}(\cdot) \in P_{n}\left(\sigma_{n}^{+}\right) E_{n}^{\beta}$ и $z_{n}(\cdot) \in\left(I_{n}-P_{n}\left(\sigma_{n}^{+}\right)\right) E_{n}^{\beta}$. Запишем

$$
\begin{aligned}
& H_{n}\left(w_{n}, z_{n}\right)=P_{n}\left(\sigma_{n}^{+}\right)\left(f_{n}\left(w_{n}+z_{n}+u_{n}^{*}\right)-f_{n}\left(u_{n}^{*}\right)-f_{n}^{\prime}\left(u_{n}^{*}\right)\left(w_{n}+z_{n}\right)\right), \\
& G_{n}\left(w_{n}, z_{n}\right)=\left(I_{n}-P_{n}\left(\sigma_{n}^{+}\right)\right)\left(f_{n}\left(w_{n}+z_{n}+u_{n}^{*}\right)-f_{n}\left(u_{n}^{*}\right)-f_{n}^{\prime}\left(u_{n}^{*}\right)\left(w_{n}+z_{n}\right)\right) .
\end{aligned}
$$

Таким образом, имеем $H_{n}(0,0)=0, G_{n}(0,0)=0$. Из непрерывной дифференцируемости функций $H_{n}$ и $G_{n}$ и оценок

$$
\begin{cases}\left\|e^{t A_{u_{n}^{*}, n}} z_{n}\right\|_{E_{n}^{\beta}} \leqslant M_{2} e^{-\gamma t}\left\|z_{n}\right\|_{E_{n}^{\beta},} & t \geqslant 0, \\ \left\|e^{t A_{u_{n}^{*}, n}} z_{n}\right\|_{E_{n}^{\beta}} \leqslant M_{2} t^{-\beta} e^{-\gamma t}\left\|z_{n}\right\|_{E_{n}}, & t>0, \\ \left\|e^{t A_{u_{n}^{*}, n}} w_{n}\right\|_{E_{n}^{\beta}} \leqslant M_{2} e^{\gamma_{1} t}\left\|w_{n}\right\|_{E_{n}^{\beta},} & t \leqslant 0,\end{cases}
$$

которые выполняются равномерно по $n$, условия аналитичности $C_{0}$-полугрупп $e^{t A_{u_{n}^{*}, n}}$ и условия $\Delta_{c c} \neq \varnothing$ получим, что для заданного $\rho>0$ существуют такие $n_{0}>0$ и $\delta>0$, что если

$$
\left\|w_{n}\right\|_{P_{n}\left(\sigma_{n}^{+}\right) E_{n}^{\beta}}+\left\|z_{n}\right\|_{\left(I_{n}-P_{n}\left(\sigma_{n}^{+}\right)\right) E_{n}^{\beta}}<\delta
$$

и $n \geqslant n_{0}$, то

$$
\begin{gathered}
\left\|H_{n}\left(w_{n}, z_{n}\right)\right\|_{P_{n}\left(\sigma_{n}^{+}\right) E_{n}} \leqslant \rho, \quad\left\|G_{n}\left(w_{n}, z_{n}\right)\right\|_{E_{n}} \leqslant \rho, \\
\left\|H_{n}\left(w_{n}, z_{n}\right)-H_{n}\left(\tilde{v}_{n}, \tilde{z}_{n}\right)\right\|_{P_{n}\left(\sigma_{n}^{+}\right) E_{n}} \leqslant \rho\left(\left\|w_{n}-\tilde{w}_{n}\right\|_{P_{n}\left(\sigma_{n}^{+}\right) E_{n}^{\beta}}+\left\|z_{n}-\tilde{z}_{n}\right\|_{\left(I_{n}-P_{n}\left(\sigma_{n}^{+}\right)\right) E_{n}^{\beta}}\right), \\
\left\|G_{n}\left(w_{n}, z_{n}\right)-G_{n}\left(\tilde{w}_{n}, \tilde{z}_{n}\right)\right\|_{E_{n}} \leqslant \rho\left(\left\|w_{n}-\tilde{w}_{n}\right\|_{P_{n}\left(\sigma_{n}^{+}\right) E_{n}^{\beta}}+\left\|z_{n}-\tilde{z}_{n}\right\|_{\left.\left(I_{n}-P_{n}\left(\sigma_{n}^{+}\right)\right) E_{n}^{\beta}\right) .}\right.
\end{gathered}
$$


Тот факт, что мы можем выбрать $\rho$ и $\delta$ равномерно по $n \geqslant n_{0}$ удовлетворяющими приведенным выше неравенствам, является ключевым для вывода о том, что локально устойчивые многообразия определены в малой «окрестности» точки равновесия $u_{n}^{*}$ равномерно по $n \geqslant n_{0}$.

Из условий $\left(\mathrm{B}_{1}\right)$ и $\Delta_{c c} \neq \varnothing$ вытекает, что $P_{n}\left(\sigma_{n}^{+}\right) \stackrel{\mathcal{P P}}{\longrightarrow} P\left(\sigma^{+}\right)$компактно; следовательно, как было показано в [79,89], спектр $\sigma\left(A_{u_{n}^{*}, n}^{+}\right)$аппроксимирует спектр $\sigma\left(A_{u^{*}}^{+}\right)$и $\operatorname{dim} P_{n}\left(\sigma_{n}^{+}\right)=\operatorname{dim} P\left(\sigma^{+}\right)$ при $n \geqslant n_{0}$.

Введем обозначение

$$
\Omega^{\alpha}=\left\{v(\cdot) \in C\left([0, \infty) ; E^{\beta}\right):\|v(t)\|_{E^{\beta}}=O\left(t^{-\alpha}\right) \text { при } t \rightarrow \infty\right\},
$$

где норма $v(\cdot) \in \Omega^{\alpha}$ равна

$$
\|v\|_{\Omega^{\alpha}}=\sup _{t \in[0, \infty)} \frac{\|v(t)\|_{E^{\beta}}}{E_{\alpha}\left(-\gamma t^{\alpha}\right)}<\infty
$$

для некоторого $\gamma>0$.

Теорема 6.3.3 (см. [222]). Предположим, что оператор А порождает аналитическую и компактную $C_{0}$-полугруппу и выполнены условия (F1) и (6.1.2). Тогда оператор

$$
K: \Omega^{\alpha} \rightarrow \Omega^{\alpha}, \quad(K u)(t) \equiv S_{\alpha}(t, A) u^{0}+\int_{0}^{t} P_{\alpha}(t-s, A) f(u(s)) d s, \quad t \in[0, \infty),
$$

компактен.

Доказательство. Пусть $\left\{u_{k}(\cdot)\right\}$ - такое множество функций $u_{k}(\cdot) \in \Omega^{\alpha}$, что $\left\|u_{k}(\cdot)\right\|_{\Omega^{\alpha}} \leqslant$ const, $k \in \mathbb{N}$. В силу условия (F1) функция $f(\cdot)$ ограничена, так что множество функций $\left\{\left(K u_{k}\right)(\cdot)\right\}$ равномерно ограничено; здесь $\left(K u_{k}\right)(\cdot) \in \Omega^{\alpha}$. Пусть $T$ таково, что

$$
\left\|f\left(u_{k}(t)\right)\right\| \leqslant \varepsilon, \quad t \geqslant T .
$$

Для $0<t_{1}<t_{2} \leqslant T$ имеем

$$
\begin{aligned}
& \left\|\left(K u_{k}\right)\left(t_{2}\right)-\left(K u_{k}\right)\left(t_{1}\right)\right\|_{E^{\beta}}= \\
& =\left\|(-A)^{\beta}\left(S_{\alpha}\left(t_{2}, A\right) u_{k}^{0}-S_{\alpha}\left(t_{1}, A\right) u_{k}^{0}+\int_{0}^{t_{2}} P_{\alpha}\left(t_{2}-s, A\right) f\left(u_{k}(s)\right) d s-\int_{0}^{t_{1}} P_{\alpha}\left(t_{1}-s, A\right) f\left(u_{k}(s)\right) d s\right)\right\| \leqslant \\
& \leqslant\left\|\left(S_{\alpha}\left(t_{2}, A\right)-S_{\alpha}\left(t_{1}, A\right)\right)(-A)^{\beta} u_{k}^{0}\right\|+ \\
& \quad+\int_{0}^{t_{1}-\delta}\left\|(-A)^{\beta}\left(P_{\alpha}\left(t_{2}-s, A\right)-P_{\alpha}\left(t_{1}-s, A\right)\right)\right\| \cdot\left\|f\left(u_{k}(s)\right)\right\| d s+ \\
& \quad+\int_{t_{1}-\delta}^{t_{1}}\left\|(-A)^{\beta}\left(P_{\alpha}\left(t_{2}-s, A\right)-P_{\alpha}\left(t_{1}-s, A\right)\right)\right\| \cdot\left\|f\left(s, u_{k}(s)\right)\right\| d s+ \\
& \leqslant c\left\|S_{\alpha}\left(t_{2}, A\right)-S_{\alpha}\left(t_{1}, A\right)\right\|+\bar{M}\left(t_{1}-\delta\right) \sup _{s \in\left[0, t_{1}-\delta\right]}\left\|(-A)^{\beta}\left(P_{\alpha}\left(t_{2}-s, A\right)-P_{\alpha}\left(t_{1}-s, A\right)\right)\right\|+ \\
& \quad+\int_{t_{1}}^{t_{2}}\left\|(-A)^{\beta} P_{\alpha}\left(t_{2}-s, A\right)\right\| \cdot\left\|f\left(s, u_{k}(s)\right)\right\| d s \leqslant \\
& \quad+2 M \bar{M} \delta+M \bar{M}\left(t_{2}-t_{1}\right)^{\alpha(1-\beta)} \rightarrow 0
\end{aligned}
$$


при $\left|t_{2}-t_{1}\right| \rightarrow 0$. Из леммы 6.2 .3 , следует, что

$$
\left\|\int_{T}^{t} P_{\alpha}(t-s, A) f(u(s)) d s\right\| \leqslant C \varepsilon \quad \text { при } t \geqslant T .
$$

Это означает, что семейство $\left\{\left(K u_{k}\right)(\cdot)\right\}$ равностепенно непрерывно. Докажем, что последовательность $\left\{\left(K u_{k}\right)(t)\right\}$ является относительно компактной для любого $t>0$. Из теоремы 6.3 .1 следует, что $S_{\alpha}(t, A)$ и $P_{\alpha}(t, A)$ компактны для любого $t>0$. Из результатов [264], следует, что

$$
\int_{0}^{T} P_{\alpha}(t-s, A) f(u(s)) d s
$$

компактно для любого конечного $T$ и

$$
\left\|\int_{T}^{t} P_{\alpha}(t-s, A) f(u(s)) d s\right\|
$$

достаточно мало при больших $T$. Таким образом,

$$
\left\{\int_{0}^{t}(-A)^{\beta} P_{\alpha}(t-s, A) f\left(u_{k}(s)\right) d s\right\}
$$

является относительно компактным для любого $t>0$. Очевидно, множество (6.3.8) является относительно компактным также и при $t=0$. Это означает, что для любого $t$ множество $\left\{\left(K u_{k}\right)(t)\right\}$ является относительно компактным в $E^{\beta}$. Из обобщенной теоремы Арцела-Асколи вытекает, что $\left\{\left(K u_{k}\right)(t)\right\}$ - компактное множество в $E^{\beta}$ и, следовательно, $\left\{\left(K u_{k}\right)(\cdot)\right\}$ компактно в $\Omega^{\alpha}$. Таким образом, $K$ - компактный оператор.

Введем операторы

$$
K_{n}: \Omega_{n}^{\alpha} \rightarrow \Omega_{n}^{\alpha}, \quad\left(K_{n} u_{n}\right)(t) \equiv S_{\alpha}\left(t, A_{n}\right) u_{n}^{0}+\int_{0}^{t} P_{\alpha}\left(t-s, A_{n}\right) f_{n}\left(u_{n}(s)\right) d s, \quad t \in[0, \infty),
$$

где

при некотором $\gamma>0$.

$$
\Omega_{n}^{\alpha} \equiv\left\{u_{n}(\cdot) \in C\left([0, \infty) ; E_{n}^{\beta}\right):\left\|u_{n}\right\|_{F_{n}}=\sup _{t \in[0, \infty)} \frac{\left\|u_{n}(t)\right\|_{E_{n}^{\beta}}}{E_{\alpha}\left(-\gamma t^{\alpha}\right)}<\infty\right\}
$$

Теорема 6.3.4. Пусть выполнены условия (F1)-(F3), $\left(\mathrm{B}_{1}\right)$ и $\Delta_{c c} \neq \varnothing$. Тогда сходимость $K_{n} \stackrel{\mathcal{P}^{\beta} \mathcal{P}^{\beta}}{\longrightarrow} K$ при $u_{n}^{0} \stackrel{\mathcal{P}^{\beta}}{\longrightarrow} u^{0}$ компактна.

Доказательство. Операторы $K_{n}$ компактны. Если $u_{n}(\cdot) \stackrel{\mathcal{P}^{\beta}}{\longrightarrow} u(\cdot)$, то из нашего предположения в (6.3.1) следует, что $f_{n}\left(u_{n}(t)\right) \stackrel{\mathcal{P}}{\longrightarrow} f(u(t))$ равномерно по $t \in[0, \infty)$. Получаем

$$
\begin{aligned}
\sup _{t \in[0, T]} \|\left(K_{n} u_{n}\right)(t) & -p_{n}^{\beta}(K u)(t)\left\|_{E_{n}^{\beta}} \leqslant \sup _{t \in[0, \infty)}\right\| S_{\alpha}\left(t, A_{n}\right) u_{n}^{0}-p_{n}^{\beta} S_{\alpha}(t, A) u^{0} \|_{E_{n}^{\beta}}+ \\
& +\sup _{t \in[0, \infty)}\left\|\int_{0}^{t} P_{\alpha}\left(t-s, A_{n}\right) f_{n}\left(s, u_{n}(s)\right) d s-p_{n}^{\beta} \int_{0}^{t} P_{\alpha}(t-s, A) f(u(s)) d s\right\|_{E_{n}^{\beta}} \rightarrow 0 .
\end{aligned}
$$

Таким образом, имеем $K_{n} \stackrel{\mathcal{P}^{\beta} \mathcal{P}^{\beta}}{\longrightarrow} K$. Пусть $\left\{u_{n}(\cdot)\right\}$ - произвольная последовательность функций $u_{n}(\cdot) \in \Omega_{n}^{\alpha}$, удовлетворяющих условию $\left\|u_{n}(\cdot)\right\|_{\Omega_{n}^{\alpha}}=O(1)$. Покажем, что

$$
\mu\left(\left\{\left(K_{n} u_{n}\right)(t)\right\}\right)=0 \quad \text { для всех } t \in[0, \infty) .
$$


Действительно,

$$
\begin{aligned}
& \mu\left(\left\{\left(-A_{n}\right)^{\beta}\left(K_{n} u_{n}\right)(t)\right\}\right) \leqslant \\
& \leqslant \mu\left(\left\{S_{\alpha}\left(t, A_{n}\right)\left(-A_{n}\right)^{\beta} u_{n}^{0}\right\}\right)+\mu\left(\left\{\int_{0}^{t}\left(-A_{n}\right)^{\beta} P_{\alpha}\left(t-s, A_{n}\right) f_{n}\left(u_{n}(s)\right) d s\right\}\right) \leqslant \\
& \leqslant \mu\left(\left\{S_{\alpha}\left(t, A_{n}\right)\left(-A_{n}\right)^{\beta} u_{n}^{0}\right\}\right)+\mu\left(\left\{\int_{0}^{t-\delta}\left(-A_{n}\right)^{\beta} P_{\alpha}\left(t-s, A_{n}\right) f_{n}\left(u_{n}(s)\right) d s\right\}\right)+ \\
& \quad+\sup _{t \in[0, \infty)}\left\|\int_{t-\delta}^{t} P_{\alpha}\left(t-s, A_{n}\right) f_{n}\left(u_{n}(s)\right) d s\right\|_{E_{n}^{\beta}} .
\end{aligned}
$$

Очевидно, первое слагаемое равно нулю. Поскольку

по лемме 6.2 .3 и

$$
\left\|P_{\alpha}\left(t, A_{n}\right)\right\|_{B\left(E_{n}^{\beta}\right)} \leqslant O\left(\frac{1}{t^{1-\alpha(1-\beta)}}\right)
$$

$$
\sup _{t \in[0, T]}\left\|f_{n}\left(u_{n}(t)\right)\right\|_{E_{n}} \leqslant \bar{M}
$$

заключаем, что третье сланаемое можно сделать меньше наперед заданного $\varepsilon$ за счет выбора достаточно малого $\delta$. Из условия (F3) следует, что семейство $\left\{f_{n}\left(u_{n}(s)\right)\right\}$ равностепенно непрерывно на $[0, t-\delta]$. Известно, что семейство $\left\{\left(-A_{n}\right)^{\beta} P_{\alpha}\left(t-s, A_{n}\right)\right\}$ также равностепенно непрерывно на $[0, t-\delta]$. Таким образом,

$$
\mu\left(\left\{\int_{0}^{t-\delta}\left(-A_{n}\right)^{\beta} P_{\alpha}\left(t-s, A_{n}\right) f_{n}\left(u_{n}(s)\right) d s\right\}\right)=0 .
$$

Следовательно, $\left\{\left(K_{n} u_{n}\right)(\cdot)\right\}$ является $P^{\beta}$-компактным и потому $K_{n} \stackrel{\mathcal{P}^{\beta} \mathcal{P}^{\beta}}{\longrightarrow} K$ компактно.

Теперь рассмотрим сходимость решений уравнений

$$
\left(K_{n} u_{n}\right)(t)=u_{n}(t)
$$

к решению уравнения

$$
(K u)(t)=u(t)
$$

Теорема 6.3.5 (см. [222]). Пусть $\Delta_{c c} \neq \varnothing$. Предположим, что выполнены условия (A), $\left(\mathrm{B}_{1}\right)$, (F1)-(F3) u $f_{n}\left(x_{n}\right) \stackrel{\mathcal{P}}{\longrightarrow} f(x)$ при $x_{n} \stackrel{\mathcal{P}^{\beta}}{\longrightarrow} x$. Тогда $u_{n}(t) \stackrel{\mathcal{P}^{\beta}}{\longrightarrow} u(t)$ при $u_{n}^{0} \stackrel{\mathcal{P}^{\beta}}{\longrightarrow} u^{0}$ равномерно по $t \in[0, \infty)$ для решений уравнений (6.3.10) и (6.3.11) соответственно.

Доказательство. Из предположений относительно $f(\cdot)$ следует, что оператор $K$ не имеет неподвижных точек на границе $\partial Q_{r}$ (это вытекает из единственности решения), где

$$
Q_{r}=\left\{u(\cdot): \sup _{t \in[0, \infty)}\left\|u(t)-u^{*}\right\|_{E_{n}^{\beta}}<r\right\} .
$$

Поскольку семейства $S_{\alpha}(\cdot, A)$ и $P_{\alpha}(\cdot, A)$ аналитичны и компактны, доказательство того факта, что $\gamma\left(I-K ; \partial Q_{r}\right)=1$, буквально повторяет доказательство теоремы 14.2.3 в [262]. Из результатов [259] следует, что

$$
\gamma\left(I-K ; \partial Q_{r}\right)=\gamma\left(I_{n}-K_{n} ; \partial Q_{n, r}\right) \quad \text { при } n \geqslant n_{0} .
$$

для $n \geqslant n_{0}$. Это означает, что найдется такая последовательность $\left\{u_{n}(\cdot)\right\}, n \in \mathbb{N}$, решений задач $(6.3 .10), u_{n}(\cdot) \in \Upsilon_{n}$, являющаяся $P^{\beta}$-компактной; поэтому можно утверждать, что $u_{n}(t) \stackrel{\mathcal{P}^{\beta}}{\longrightarrow} u(t)$ равномерно по $t \in[0, \infty)$ при $n \rightarrow \infty$. 
Теорема 6.3.6 (см. [222]). Пусть выполнены предположения теоремы 6.3.3. Тогда оператор $\Theta(\eta, \cdot): \Upsilon \rightarrow \Upsilon$ компактен.

Доказательство. Во-первых, напомним, что проектор $P\left(\sigma^{+}\right)$конечномерен. Для любого $P_{j}$ функция

$$
\int_{0}^{t} P_{\alpha}\left(t-s, A_{u^{*}}^{+}\right) P_{j} H(w(s), z(s)) d s-E_{\alpha}\left(t^{\alpha} A_{u^{*}}^{+}\right) \int_{0}^{\infty} Q\left(e^{-\lambda_{1}^{1 / \alpha} \tau}, \tau\right) P_{j} H(w(\tau), z(\tau)) d \tau
$$

равномерно ограниченна и равностепенно непрерывна при $t>0$. По теореме Арцела-Асколи часть оператора $\Theta$, соответствующая $P\left(\sigma^{+}\right)$, компактна. Чтобы доказать компактность части

$$
E_{\alpha}\left(t^{\alpha} A_{u^{*}}^{-}\right) \eta+\int_{0}^{t} P_{\alpha}\left(t-s, A_{u^{*}}^{-}\right) G(w(s), z(s)) d s
$$

оператора $\Theta$, достаточно применить теорему 6.3.3.

6.3.1. Аппроксимация устойчивых многообразий. Сформулируем утверждение об аппроксимации устойчивых многообразий для случая дробных дифференциальных уравнений.

Теорема 6.3.7. Пусть $\Delta_{c c} \neq \varnothing$ и выполнены условия (F1)-(F3). Тогда сходимость

$$
\Theta_{n}\left(\eta_{n}, \cdot\right) \rightarrow \Theta(\eta, \cdot) \quad \text { npu } \eta_{n} \stackrel{\mathcal{P}^{\beta}}{\longrightarrow} \eta
$$

операторов $\Theta_{n}\left(\eta_{n}, \cdot\right): \Upsilon_{n} \rightarrow \Upsilon_{n}, \Theta(\eta, \cdot): \Upsilon \rightarrow \Upsilon$ компактна.

Доказательство. Напомним, что

$$
\left\|q_{n}(\cdot)\right\|_{\Upsilon_{n}}=\left\|\left(\begin{array}{c}
q_{n}^{1}(\cdot) \\
q_{n}^{2}(\cdot)
\end{array}\right)\right\|_{\Upsilon_{n}}=\sup _{t \geqslant 0}\left\|q_{n}^{1}(t)\right\|_{P_{n}\left(\sigma_{n}^{+}\right) E_{n}^{\beta}}+\sup _{t \geqslant 0}\left\|q_{n}^{2}(t)\right\|_{\left(I_{n}-P_{n}\left(\sigma_{n}^{+}\right)\right) E_{n}^{\beta}} .
$$

Таким образом, дискретная сходимость $\Upsilon_{n} \ni q_{n}(\cdot) \rightarrow q(\cdot) \in \Upsilon$ означает, что

$$
\sup _{0 \leqslant t<\infty}\left\|q_{n}^{1}(t)-p_{n}^{\beta} q^{1}(t)\right\|_{P_{n}\left(\sigma_{n}^{+}\right) E_{n}^{\beta}}+\sup _{0 \leqslant t<\infty}\left\|q_{n}^{2}(t)-p_{n}^{\beta} q^{2}(t)\right\|_{\left(I_{n}-P_{n}\left(\sigma_{n}^{+}\right)\right) E_{n}^{\beta}} \rightarrow 0 \quad \text { при } n \rightarrow \infty .
$$

Проверим, что из условия $\left\|q_{n}(\cdot)\right\| \Upsilon_{n}=O(1)$ вытекает $P^{\beta}$-компактность последовательности $\left\{\Theta_{n}\left(\eta_{n}, q_{n}(\cdot)\right)\right\}$. Применим теорему Арцела-Асколи к последовательностям

$$
\left(-A_{n}\right)^{\beta} E_{\alpha}\left(t^{\alpha} A_{u_{n}^{*}}^{-}\right) \eta_{n}+\left(-A_{n}\right)^{\beta} \int_{0}^{t} P_{\alpha}\left(t-s, A_{u_{n}^{*}}^{-}\right) G_{n}\left(q_{n}^{1}(s), q_{n}^{2}(s)\right) d s
$$

и

$$
\begin{aligned}
& \left(-A_{n}\right)^{\beta}\left(\int_{0}^{t} P_{\alpha}\left(t-s, A_{u_{n}^{*}}^{+}\right) P_{n, j} \tilde{H}_{n}\left(q_{n}^{1}(s), q_{n}^{2}(s)\right) d s-\right. \\
& \left.\quad-E_{\alpha}\left(t^{\alpha} A_{u_{n}^{*}}^{+}\right) \int_{0}^{\infty} Q\left(e^{-\lambda_{1}^{1 / \alpha} s}, s\right) P_{n, j} \tilde{H}_{n}\left(q_{n}^{1}(s), q_{n}^{2}(s)\right) d s\right) .
\end{aligned}
$$

В силу (6.3.6) имеем

$$
\left\|\left(-A_{n}\right)^{\beta} \int_{0}^{t} P_{\alpha}\left(t-s, A_{n}\right) \tilde{H}_{n}\left(q_{n}^{1}(s), q_{n}^{2}(s)\right) d s\right\| \leqslant \varepsilon \quad \text { при } t \geqslant t_{\varepsilon} .
$$


Остается применить теорему Арцела-Асколи к множествам функций

$$
\begin{gathered}
\left\{\left(-A_{n}\right)^{\beta} \int_{0}^{t} P_{\alpha}\left(t-s, A_{n}+f_{n}^{\prime}\left(u_{n}^{*}\right)\right) P_{n}\left(\sigma_{n}^{+}\right) \tilde{H}_{n}\left(q_{n}^{1}(s), q_{n}^{2}(s)\right) d s\right\}, \\
\left\{\left(-A_{n}\right)^{\beta} \int_{0}^{t} P_{\alpha}\left(t-s, A_{u_{n}^{*}}^{+}\right)\left(I_{n}-P_{n}\left(\sigma_{n}^{+}\right)\right) G_{n}\left(q_{n}^{1}(s), q_{n}^{2}(s)\right) d s\right\}
\end{gathered}
$$

на $\left[0, t_{\varepsilon}\right]$; это делается, как в теореме 6.3.4.

Теорема 6.3.8 (см. [222]). Пусть $\Delta_{c c} \neq \varnothing u u^{*}$ - гиперболическая точка равновесия задачи (6.1.1). Тогда для достаточно малого $\delta>0$ уравнение (6.2.7) имеет такое решение $\zeta^{*}(\cdot)$, что уравнения

$$
\zeta_{n}(t)=\Theta_{n}\left(\eta_{n}, \zeta_{n}(t)\right)
$$

имеют решения $\zeta_{n}^{*}(\cdot)$ при $n \geqslant n_{0} u \zeta_{n}^{*}(t) \rightarrow \zeta^{*}(t)$ равномерно по $t \in[0, \infty)$ nри $n \rightarrow \infty$.

Доказательство. Известно, что $\left|\operatorname{ind}\left(\zeta_{0}, I-\Theta\right)\right|=1$ при $\zeta_{0}=0$. Согласно [21, теорема 54.1], существуют такие $\rho, \delta>0$, что $|\operatorname{ind}(\zeta, I-\Theta)|=1$ при $\|\eta-0\|_{E^{\beta}} \leqslant \delta$ и $\|\zeta-0\|_{\Upsilon} \leqslant \rho$. По теореме 6.3.7 операторы $\Theta_{n} \rightarrow \Theta$ сходятся компактно, так что

$$
\gamma\left(I_{n}-\Theta_{n}, \partial Q_{n}\right)=\operatorname{ind}(\zeta, I-\Theta)
$$

и, следовательно, уравнение

$$
\zeta_{n}(t)=\Theta_{n}\left(\eta_{n}, \zeta_{n}(t)\right)
$$

имеет хотя бы одно решение $\zeta_{n}^{*}(\cdot)$ в любом множестве $Q_{n}$ при $n \geqslant n_{0}$. Последовательность $\left\{\zeta_{n}^{*}(\cdot)\right\}$ дискретно компактна и $\zeta_{n}^{*}(t) \rightarrow \zeta^{*}(t)$ равномерно по $t \geqslant 0$ при $n \rightarrow \infty$.

Предложение 6.3.1. Предположим, что и* - гиперболическое равновесие задачи (6.1.1) и нуль не принадлежит спектру оператора $A+f^{\prime}\left(u^{*}\right): D(A) \subset E \rightarrow E$. Пусть $\Delta_{c c} \neq \varnothing u$ $\left\|f_{n}^{\prime}\left(u_{n}\right)-f_{n}^{\prime}\left(p_{n}^{\beta} u^{*}\right)\right\| \rightarrow 0$ nри $\left\|u_{n}-p_{n}^{\beta} u^{*}\right\|_{E_{n}^{\beta}} \rightarrow 0$. Тогда существуют такие $\delta>0$ и $n_{0}>0$, что $u_{n}^{*}$ имеет локальное устойчивое многообразие $W_{l o c}^{s t}\left(u_{n}^{*}\right) \subset E_{n}^{\beta}$ для любого $n \geqslant n_{0}$. Если обозначить его $\delta$-часть через

$$
W_{n, \delta}^{s t}\left(u_{n}^{*}\right)=\left\{v_{n}(\cdot) \in W_{l o c}^{s t}\left(u_{n}^{*}\right):\left\|v_{n}(t)-u_{n}^{*}\right\|_{E_{n}^{\beta}}<\delta, t \geqslant 0\right\}, \quad n \geqslant n_{0},
$$

mо $W_{n, \delta}^{s t}\left(u_{n}^{*}\right)$ сходится $\kappa W_{\delta}^{s t}\left(u^{*}\right)$ nри $n \rightarrow \infty$, т.е. для любого $t \in[0, \infty)$

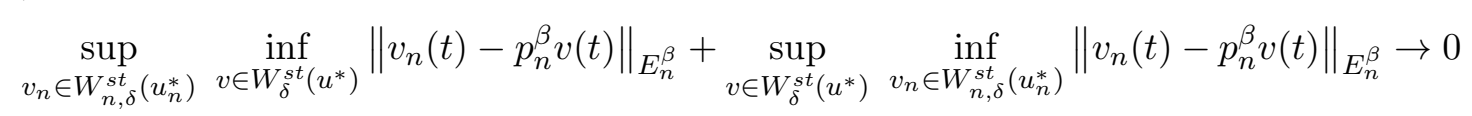

nрu $n \rightarrow \infty$.

Доказательство. Покажем, что для достаточно малого $\rho>0$ найдется устойчивое многообразие для $u_{n}^{*}$ :

$$
W_{n}^{\text {st }}=\left\{\left(w_{n}(\cdot), z_{n}(\cdot)\right): z_{n}(\cdot) \in\left(I_{n}-P_{n}\left(\sigma_{n}^{+}\right)\right) E_{n}^{\beta}, w_{n}(\cdot) \in P_{n}\left(\sigma_{n}^{+}\right) E_{n}^{\beta}\right\} .
$$

Из (6.3.6) для вектора $\zeta_{n}(t)=\left(\begin{array}{c}w_{n}(t) \\ z_{n}(t)\end{array}\right)$ получаем, что найдется такое положительное $\delta$, что операторы $\Theta_{n}\left(\eta_{n}, \cdot\right)$ дифференцируемы по Фреше в шарах $\mathcal{U}_{E_{n}^{\beta}}\left(p_{n}^{\beta} \zeta^{*} ; \delta\right)$ и для любого $\varepsilon>0$ найдется такое $\delta_{\varepsilon}>0$, что

при

$$
\left\|\Theta_{n}^{\prime}\left(\eta_{n}, \zeta_{n}\right)-\Theta_{n}^{\prime}\left(\eta_{n}, p_{n}^{\beta} \zeta^{*}\right)\right\| \leqslant \varepsilon
$$

$$
\left\|\zeta_{n}(\cdot)-p_{n}^{\beta} \zeta^{*}(\cdot)\right\|_{\Upsilon_{n}} \leqslant \delta_{\varepsilon} .
$$


Ясно также, что выполнены условия

$$
\left\|p_{n}^{\beta} \zeta^{*}(\cdot)-\Theta_{n}\left(\eta_{n}, p_{n}^{\beta} \zeta^{*}(\cdot)\right)\right\|_{\Upsilon_{n}} \rightarrow 0, \quad\left\|\left(I_{n}-\Theta_{n}^{\prime}\left(\eta_{n}, p_{n}^{\beta} \zeta^{*}(\cdot)\right)\right)^{-1}\right\| \leqslant \text { const } .
$$

Тогда согласно [259, теорема 2], получаем, что существуют такие $n_{0} \in \mathbb{N}$ и $0<\delta_{0} \leqslant \delta$, что уравнения (6.3.4) имеют при $n \geqslant n_{0}$ единственные решения $\zeta_{n}^{*}(\cdot)$ в шарах $\mathcal{U}_{E_{n}^{\beta}}\left(p_{n}^{\beta} \zeta^{*} ; \delta_{0}\right)$ и $\zeta_{n}^{*}(\cdot) \rightarrow \zeta^{*}(\cdot)$ при $n \rightarrow \infty$.

\section{СПИСОК ЛИТЕРАТУРЫ}

1. Антонюк A. В., Кочубей A. Н., Пискарев С. И. О компактности и равномерной непрерывности разрешающего семейства для уравнения с дробными производными// Допов. нац. акад. наук Укр. - 2014. - № 6. - С. 7-12.

2. Бабенко Ю. И. Метод дробного дифференцирования в прикладных задачах теории тепломассообмена. - НПО «Профессионал», 2009.

3. Баскаков А. Г. Линейные дифференциальные операторы с неограниченными операторными коэффициентами и полугруппы разностных операторов// Мат. заметки. - 1996. - 59, № 6. - С. $811-820$.

4. Баскаков А. Г. Спектральный анализ линейных дифференциальных операторов и полугруппы разностных операторов, II// Диффер. уравн. - 2001. - 37, № 1. - С. 3-11.

5. Баскаков А. Г. О дифференциальных и разностных операторах Фредгольма// Докл. РАН. - 2007. - 416. - C. $156-160$.

6. Баскаков А. Г. Исследование дифференциальных уравнений с неограниченными операторными коэффициентами методами теории операторов. - Воронеж: Издательский дом ВГУ, 2017.

7. Баскаков А. Г., Калужина Н. С., Поляков Д.М. Медленно меняющиеся на бесконечности полугруппы операторов// Изв. вузов. Мат. - 2014. - № 7. - С. 3-14.

8. Баскаков А. Г., Пастухов А. И. Спектральный анализ оператора взвешенного сдвига с неограниченными операторными коэффициентами// Сиб. мат. ж. - 2001. - 42, № 6. - С. 1231-1243.

9. Бобылев Н. А., Красносельский М. А. Функционализация параметра и теорема родственности для автономных систем// Диффер. уравн. - 1970. - 6, № 11. - С. 1946-1952.

10. Бобылев Н. А., Красносельский М. А. Об аппроксимации авторелаксаций в системах автоматического управления// Докл. АН СССР. - 1983. - 272, № 2. - С. 267-271.

11. Васильев В. В., Крейн С. Г., Пискарев С. И. Полугруппы операторов, косинус оператор-функции и линейные дифференциальные уравнения// Итоги науки и техн. Сер. Мат. анализ. - 1990. - 28. C. $87-202$.

12. Васильев B. В., Пискарев С. И. Дифференциальные уравнения в банаховом пространстве. Теория полугрупп операторов. - М.: Изд-во МГУ, 1996.

13. Васильев B. В., Пискарев С. И. Библиографический указатель по теме «Дифференциальные уравнения в абстрактных пространствах». - М.: НИВЦ МГУ, 2001.

14. Васильев В. В., Пискарев С. И., Селиванова Н. Ю. Проинтегрированные полугруппы, $C$-полугруппы и их приложения// Итоги науки и техн. Совр. мат. прилож. Темат. обзоры. - 2017. - 131. - С. 3-109.

15. Вишик M. И., Чепъжов В. В. Аппроксимация траекторий, лежащих на глобальном аттракторе гиперболического уравнения с быстро осциллирующей по времени внешней силой// Мат. сб. — 2003. - 194, № 9. - С. 3-30.

16. Гохберг И. Ц., Крейн М. Г. Введение в теорию линейных несамосопряженных операторов в гильбертовом пространстве. - М.: Наука, 1965.

17. Гурова И. Н. О методах полудискретизации для квазилинейных уравнений с некомпактной полугруппой// Изв. вузов. Мат. - 2000. - № 4. - С. 60-65.

18. Каток А. Б., Хасселблат Б. Введение в современную теорию динамических систем. - М.: Факториал, 1999.

19. Кокурин М. М. О единственности решения обратной задачи Коши для дифференциального уравнения с дробной производной в банаховом пространстве// Изв. вузов. Мат. - 2013. — № 12. - С. 19-35. 
20. Корнев А. А. Об аппроксимации аттракторов полудинамических систем// Мат. сб. - 2001. - 192 , № 10 . - C. $19-32$.

21. Красносельский М. А., Забрейко П. П. Геометрические методы нелинейного анализа. - М.: Наука, 1975.

22. Красносельский М. А., Забрейко П. П., Пустыльник Е. Е., Соболевский П. Е. Интегральные операторы в пространствах суммируемых функций. - М.: Наука, 1966.

23. Крейн С. Г. Линейные дифференциальные уравнения в банаховом пространстве. - М.: Наука, 1967.

24. Пискарев С. И. Оценки погрешности при аппроксимации полугрупп операторов дробями Паде// Изв. вузов. Мат. - 1979. - № 4. - С. 33-38.

25. Пискарев С. И. Сходимость разностных схем при решении нелинейных параболических уравнений// Мат. заметки. - 1988. - 44, № 1. - С. 112-123.

26. Пискарев С. И. Дифференциальные уравнения в банаховом пространстве и их аппроксимация. Изд-во МГУ, 2005.

27. Попов А. Ю., Седлецкий А. М. Распределение корней функций Миттаг-Леффлера// Совр. мат. Фундам. направл. - 2011. - 40. - С. 3-171.

28. Самко С. Г., Килбас А. А., Маричев О. И. Интегралы и производные дробного порядка и некоторые их приложения. - Минск: Наука и техника, 1987.

29. Соболевский П. Е. Теория полугрупп и устойчивость разностных схем// в кн.: Теория операторов в функциональных пространствах. - Новосибирск, 1975. - С. 304-337.

30. Соболевский П. Е. Некоторые свойства решений дифференциальных уравнений в дробных пространствах // Тр. Ин-та мат. Воронеж. гос. ун-та. - 1975. - № 74. - С. 68-76.

31. Федоров B. E., Романова E. A. Неоднородное эволюционное уравнение дробного порядка в секториальном случае// Итоги науки и техн. Совр. мат. прилож. Темат. обзоры. - 2018. - 149. - C. $103-112$.

32. Agase S. B., Raghavendra V. Existence of mild solutions of semilinear differential equations in Banach spaces// Indian J. Pure Appl. Math. — 1990. — 21, № 9. — P. 813-821.

33. Ahues M., Piskarev S. Spectral approximation of weakly singular integral operators. I. Convergence theory// Proc. 4 Int. Conf. "Integral Methods in Science and Engineering" (Oulu, Finland, June 17-20, 1996). - Longman, 1996.

34. Akhmerov R. R., Kamenskii M. I., Potapov A. S., Rodkina A. E., Sadovskii B. N. Measures of Noncompactness and Condensing Operators. — Basel: Birkhäuser-Verlag, 1992.

35. Akrivis G. Stability of implicit and implicit-explicit multistep methods for nonlinear parabolic equations// IMA J. Numer. Anal. — 2018. — 38, № 4. - P. 1768-1796.

36. Alikhanov A. A. A new difference scheme for the time fractional diffusion equation// J. Comput. Phys. 2015. - 280. - P. 424-438.

37. Alkhayuon H. M., Ashwin P. Rate-induced tipping from periodic attractors: partial tipping and connecting orbits / / Chaos. - 2018. - 28, № 3. - 033608 .

38. Alouges F., Debussche A. On the qualitative behavior of orbits of a parabolic partial differential equation and its discretizatin in the neighborhood of a hyperbolic fixed point// Numer. Funct. Anal. Optim. 1991. - 12. - P. 253-269.

39. Alvarez E., Lizama C. Weighted pseudo almost periodic solutions to a class of semilinear integrodifferential equations in Banach spaces// Adv. Differ. Eqs. - 2015. - 2015. - 31.

40. Amann H. Existence and regularity for semilinear parabolic evolution equations// Ann. Scu. Norm. Sup. Pisa Cl. Sci. - 1984. - 11, № 4. - P. 593-675.

41. Amann H. Linear and Quasilinear Parabolic Problems. Vol. I. Abstract Linear Theory. - Basel: Birkhäuser-Verlag, 1995.

42. Ambrosetti A., Prodi G. A Primer of Nonlinear Analysis. - Cambridge: Cambridge Univ. Press, 1995.

43. Amir B., Maniar L. Existence and asymptotic behavior of solutions of semilinear Cauchy problems with nondense domain via extrapolation spaces// Rend. Circ. Mat. Palermo, II. Ser. — 2000. — 49, № 3. — P. 481-496.

44. Anselone P. M. Collectively Compact Operator Approximation Theory and Applications to Integral Equations. — Englewood Cliffs, New Jersey: Prentice-Hall, 1971. 
45. Aparicio R., Keyantuo $V$. Well-posedness of degenerate integro-differential equations in function spaces// Electr. J. Differ. Eqs. — 2018. - 2018. - 79.

46. Appell J. Measures of noncompactness, condensing operators and fixed points: An application-oriented survey// Fixed Point Theory. — 2005. - 6, № 2. — P. 157-229.

47. Appell J., De Pascale E., Vignoli A. Nonlinear Spectral Theory. 2004.

48. Appell J., Zabrejko P., Nonlinear Superposition Operators. - Cambridge: Cambridge Univ. Press, 1990.

49. Aràndiga F., Caselles V. On strongly stable approximations// Rev. Mat. Univ. Complut. Madrid. — 1994. - 7, № 2. - P. 207-217.

50. Arendt W., Batty C. J., Hieber M. K., Neubrander F. Vector-Valued Laplace Transforms and Cauchy Problems. - Basel: Birkhäuser, 2001.

51. Arrieta J. M., Carvalho A. N., Lozada-Cruz G. Dynamics in dumbbell domains. I. Continuity of the set of equilibria// J. Differ. Eqs. - 2006. - 231. - P. 551-597.

52. Arrieta J. M., Carvalho A. N., Rodriguez-Bernal A. Parabolic problems with nonlinear boundary conditions and critical nonlinearities// J. Differ. Eqs. - 1999. - 156, № 2. - P. 376-406.

53. Arrieta J. M., Carvalho A. N., Rodriguez-Bernal A. Upper semicontinuity for attractors of parabolic problems with localized large diffusion and nonlinear boundary conditions// J. Differ. Eqs. — 2000. — 168, № 1. - P. 33-59.

54. Arrieta J. M., Carvalho A. N., Rodriguez-Bernal A. Attractors of parabolic problems with nonlinear boundary conditions. Uniform bounds// Commun. Partial Differ. Eqs. — 2000. — 25, № 1-2. — P. 1-37.

55. Ashyralyev A. A note on fractional derivatives and fractional powers of operators// J. Math. Anal. Appl. - 2009. - 357. - P. 232-236.

56. Ashyralyev A., Cuevas C., Piskarev S. On well-posedness of difference schemes for abstract elliptic problems in $L^{p}([0, T] ; E)$ spaces // Numer. Funct. Anal. Optim. - 2008. — 29, № 1-2. — P. 43-65.

57. Ashyralyev A., Martinez M., Pastor J., Piskarev S. On well-posedness of abstract hyperbolic problems in function spaces// Proc. WSPC. - 2009. - P. 679-688.

58. Ashyralyev A., Pastor J., Piskarev S., Yurtsever H. A. Second-order equations in functional spaces: qualitative and discrete well-posedness// J. Abstr. Appl. Anal. — 2015. — 948321.

59. Ashyralyev A., Piskarev S., Weis L. On well-posedness of difference schemes for abstract parabolic equations in $L^{p}([0, T] ; E)$ spaces// Numer. Funct. Anal. Optim. — 2002. — 23, № 7-8. — P. 669-693.

60. Ashyralyev A., Sobolevskii P. E. Well-Posedness of Parabolic Difference Equations. - Basel-BostonBerlin: Birkhäuser-Verlag, 1994.

61. Ashyralyev A., Sobolevskii P. E. New Difference Schemes for Partial Differential Equations. - BaselBoston-Berlin: Birkhäuser-Verlag, 2004.

62. Atangana A. Derivative with a New Parameter. Theory, Methods and Applications. — Amsterdam: Elsevier/Academic Press, 2016.

63. Atangana A. Fractional Operators with Constant and Variable Order with Application to Geo-Hydrology. - Amsterdam: Elsevier/Academic Press, 2018.

64. Atangana A. Nonvalidity of index law in fractional calculus: A fractional differential operator with Markovian and non-Markovian properties// Phys. A. - 2018. - 505. - P. 688-706.

65. Atangana A., Goufo E. F. D. Conservatory of Kaup-Kupershmidt equation to the concept of fractional derivative with and without singular kernel// Acta Math. Appl. Sin. — 2018. — 34, № 2. — P. 351-361.

66. Bajlekova E. G. Fractional evolution equations in Banach spaces/ Ph.D. Thesis - Eindhoven Univ. Technology, 2001.

67. Bakaev N. Yu. Linear Discrete Parabolic Problems. - Amsterdam: North-Holland, 2006.

68. Bakaev N., Thomee V., Wahlbin L. B. Maximum-norm estimates for resolvents of elliptic finite element operators// Math. Comp. - 2003. - 72. — P. 1597-1610.

69. Banas J., Goebel K. Measures of Noncompactness in Banach Spaces. - New York-Basel: Marcel Dekker, 1980. 
70. Barbu V., Pavel N. On the invertibility of $I+\exp (-t A)$ with $A$ maximal monotone// Proc. World Congr. Nonlin. Anal., (Tampa, Florida, August 19-26, 1992). — de Gruyter, 1992. — P. 2231-2237.

71. Baskakov A. G., Krishtal I. A. Spectral analysis of operators with the two-point Bohr spectrum// J. Math. Anal. Appl. - 2005. - 308, № 2. - P. 420-439.

72. Baskakov A. G., Sintyaev Yu. N. Finite-difference operators in the study of differential operators: solution estimates// J. Differ. Eqs. — 2010. — 46, № 2. — P. 214-223.

73. Batty Charles J. K., Chill R., Tomilov Yu. Strong stability of bounded evolution families and semigroups// J. Funct. Anal. — 2002. — 193, № 1. - P. 116-139.

74. Bernardes Jr. N. C., Cirilo P. R., Darji U. B., Messaoudi A., Pujals E. R. Expansivity and shadowing in linear dynamics// J. Math. Anal. Appl. — 2018. — 461, № 1. - P. 796-816.

75. Beyn W.-J. The numerical computation of connecting orbits in dynamical systems// IMA J. Numer. Anal. - 1990. - 10, № 3. - P. 379-405.

76. Beyn W.-J. Numerical methods for dynamical systems// Proc. 4th Summer School "Advances in Numerical Analysis" (Lancaster, UK, 1990). — New York: Oxford Univ. Press, 1991. — P. 175-236.

77. Beyn W.-J. On the numerical approximation of phase portraits near stationary points// SIAM J. Numer. Anal. - 1987. - 24, № 5. - P. 1095-1113.

78. Beyn W.-J., Kolezhuk V. S., Pilyugin S. Yu. Convergence of discretized attractors for parabolic equations on the line// Zap. Nauch. Semin. LOMI - 2004. - 318. - P. 14-41.

79. Beyn W.-J., Piskarev S. I. Shadowing for discrete approximations of abstract parabolic equations// Discr. Cont. Dynam. Syst. Ser. B. - 2008. - 10, № 1. - P. 19-42.

80. Bobylev N. A., Kim J. K., Korovin S. K., Piskarev S. I. Semidiscrete approximations of semilinear periodic problems in Banach spaces// Nonlin. Anal. — 1998. — 33, № 5. - P. 473-482.

81. Bruschi S. M., Carvalho A. N., Cholewa J. W., Dlotko T. Uniform exponential dichotomy and continuity of attractors for singular perturbed damped wave equation// J. Dynam. Differ. Eqs. — 2006. — 18. — P. 767.

82. Bu S., Cai G. Solutions of second-order degenerate integro-differential equations in vector-valued function spaces// Sci. China Math. — 2013. — 56, № 5. - P. 1059-1072.

83. Bu S., Cai G. Well-posedness of second-order degenerate differential equations with finite delay// Proc. Edinb. Math. Soc. — 2017. - 60. - P. 349-360.

84. Bu S., Cai G. Well-posedness of second-order degenerate differential equations with finite delay in vectorvalued function spaces// Pac. J. Math. - 2017. — 288, № 1. - P. 27-46.

85. Bu S., Cai $G$. Well-posedness of fractional degenerate differential equations with finite delay on vectorvalued functional spaces// Math Nach. — 2018. - 291. - P. 759-773.

86. Cao Q., Pastor J., Siegmund S., Piskarev S. Approximations of parabolic equations at the vicinity of hyperbolic equilibrium point// Numer. Funct. Anal. Optim. — 2014. — 35, № 10. — P. 1287-1307.

87. Carbone V. L., Carvalho A. N., Schiabel-Silva K. Continuity of attractors for parabolic problems with localized large diffusion// Nonlin. Anal. Theory Meth. Appl. — 2008. — 68, № 3. — P. 515-535.

88. Carvalho A. N., Langa J. A., Robinson J. C. Attractors for Infinite-Dimensional Non-Autonomous Dynamical Systems. — Berlin: Springer-Verlag, 2013.

89. Carvalho A. N., Piskarev S. I. A general approximation scheme for attractors of abstract parabolic problems// Numer. Funct. Anal. Optim. — 2006. — 27, № 7-8. — P. 785-829.

90. Chatelin F. Spectral Approximation of Linear Operators. - New York: Academic Press, 1983.

91. Cholewa J. W., Dlotko T. Global Attractors in Abstract Parabolic Problems. - Cambridge: Cambridge Univ. Press, 2000.

92. Clément P., Gripenberg G., Londen S.-O. Regularity properties of solutions of fractional evolution equations// in: Evolution Equations and Their Applications in Physical and Life Sciences (Lumer G. et al., eds.). - New York: Marcel Dekker, 2001. - P. 235-246.

93. Clément P., Londen S.-O. Regularity aspects of fractional evolution equations// Rend. Ist. Mat. Univ. Trieste. - 2000. - 31, № 2. - P. 19-20.

94. Cong N. D., Doan T. S., Siegmund S., Tuan H. T. On stable manifolds for planar fractional differential equations// Appl. Math. Comput. — 2014. - 226. — P. 157--168. 
95. Cong N. D., Doan T. S., Siegmund S., Tuan H. T. On stable manifolds for fractional differential equations in high-dimensional spaces// Nonlin. Dynam. - 2016. — 86. - P. 1885-1894.

96. Crouzeix M., Larsson S., Piskarev S., Thomee V. The stability of rational approximations of analytic semigroups// BIT Numer. Math. — 1993. — 33, № 1. — P. 74-84.

97. Czaja R., Marin-Rubio P. Pullback exponential attractors for parabolic equations with dynamical boundary conditions// Taiwan. J. Math. — 2017. — 21, № 4. - P. 819-839.

98. da Prato G., Grisvard P. Sommes d'opérateus linéaires et équations différentielles opérationnelles// J. Math. Pures Appl. IX. Sér. - 1975. - 54, № 3. - P. 305-387.

99. da Prato G., Grisvard P. Équations d'évolution abstraites non linéaires de type parabolique// C. R. Acad. Sci. Paris. Sér. A. - 1976. - 283, № 9. - P. 709-711.

100. Danca M.-F., Feckan M., Kuznetsov N. V., Chen G. Complex dynamics, hidden attractors and continuous approximation of a fractional-order hyperchaotic PWC system// Nonlin. Dynam. — 2018. — 91, № 4. — P. 2523-2540.

101. Dancer E. N. Upper and lower stability and index theory for positive mappings and applications// Nonlin. Anal. Theory Meth. Appl. - 1991. - 17. - P. 205-217.

102. Deshpande A., Daftardar-Gejji V. Local stable manifold theorem for fractional systems// Nonlin. Dynam. - 2016. - 83. - P. 2435-2452.

103. Diethelem K. The Analysis of Fractional Differential Equations. - Heidelberg-Dordrecht-London-New York: Springer-Verlag, 2004.

104. Eberhardt B., Greiner G. Baillon's theorem on maximal regularity// Acta Appl. Math. — 1992. — 27. — P. $47-54$.

105. El-Borai M. M. Some probability densities and fundamental solutions of fractional evolution equations// Chaos Solitons Fract. - 2002. - 14. - P. 433-440.

106. Engel K.-J., Nagel R. One-Parameter Semigroups for Linear Evolution Equations. — Berlin: SpringerVerlag, 2000.

107. Engel K.-J., Nagel R. A Short Course on Operator Semigroups. — New York: Springer-Verlag, 2006.

108. Ergenç T., Karasözen B., Piskarev S. Approximation for semilinear Cauchy problems involving second order equations in separable Banach spaces// Nonlin. Anal. Theory Meth. Appl. — 1997. — 28. — P. 11571165.

109. Fan Z. Characterization of compactness for resolvents and its applications// Appl. Math. Comput. 2014. - 232. - P. 60-67.

110. Fan Z., Dong Q., Li G. Almost exponential stability and exponential stability of resolvent operator families// Semigroup Forum. - 2016. - 93. - P. 491-500.

111. Fujita H., Mizutani A. On the finite element method for parabolic equations. I. Approximation of holomorphic semigroups// J. Math. Soc. Jpn. — 1976. — 28, № 4. - P. 749-771.

112. Fujita H., Suzuki T. Evolution problems// in: Handbook of Numerical Analysis. — Amsterdam: NorthHolland, 1991. - P. 789-928.

113. Gao G., Sun Z. The finite difference approximation for a class of fractional sub-diffusion equations on a space unbounded domain// J. Comput. Phys. - 2013. - 236. — P. 443-460.

114. Gal C. G., Warma M. Fractional-in-Time Semilinear Parabolic Equations and Applications. — Springer, 2020.

115. Garay B. M. On structural stability of ordinary differential equations with respect to discretization methods// Numer. Math. — 1996. — 72, № 4. — P. 449-479.

116. Garay B. M., Lee K. Attractors under discretization with variable stepsize// Discr. Contin. Dynam. Syst. - 2005. - 13, № 3. - P. 827-841.

117. Gearhart L. Spectral theory for contraction semigroups on Hilbert spaces// Trans. Am. Math. Soc. 1978. - 236. - P. 385-394.

118. Goldstein J. A. Semigroups of Linear Operators and Applications. - New York: Clarendon Press, Oxford University Press, 1985.

119. Grigorieff R. D. Zur Theorie linearer approximationsregulärer Operatoren, I, II// Math. Nachr. — 1973. - 55. - P. 233-249, 251-263. 
120. Grigorieff R. D. Diskrete Approximation von Eigenwertproblemen. I. Qualitative Konvergenz// Numer. Math. - 1975. - 24. - P. 355-374.

121. Grigorieff R. D. Diskrete Approximation von Eigenwertproblemen. II. Konvergenzordnung// Numer. Math. - 1975. - 24. - P. 415-433.

122. Grigorieff R. D. Diskrete Approximation von Eigenwertproblemen. III. Asymptotische Entwicklungen// Numer. Math. - 1975/76. - 25, № 1. - P. 79-97.

123. Grigorieff R. D. Über diskrete Approximationen nichtlinearer Gleichungen. 1. Art// Math. Nachr. - 1975. - 69. - P. 253-272.

124. Grigorieff R. D. Zur Charakterisierung linearer approximationsregulärer Operatoren// in: Mathematical Papers Given on the Occasion of Ernst Mohr's 75th Birthday. — Berlin: Tech. Univ. Berlin, 1985. — P. 63-77.

125. Grigorieff R. D., Jeggle H. Approximation von Eigenwertproblemen bei nichlinearer Parameterabhängigkeit// Manuscr. Math. — 1973. - 10. — P. 245-271.

126. Guidetti D. Fractional derivative in spaces of continuous functions. - preprint, 2019.

127. Guidetti D., Karasözen B., Piskarev S. Approximation of abstract differential equations// J. Math. Sci. - 2004. - 122, № 2. - P. 3013-3054.

128. Gurova I. N. On a certain topological method for studying difference schemes// Dokl. Akad. Nauk SSSR. - 1979. - 248, № 1. - P. 25-28.

129. Gurova I. N., Kamenskii M. I. On the semidiscretization method in the problem of periodic solutions of quasilinear autonomous parabolic equations// Differ. Eqs. - 1996. — 32, № 1. — P. 106-112.

130. Hu Y., Hе J. H. On fractal space-time and fractional calculus// Thermal Sci. — 2016. — 20, № 3. — P. $773-777$.

131. Haase M. The Functional Calculus for Sectorial Operators. - Basel: Birkhäuser-Verlag, 2006.

132. Hale J. K. Asymptotic Behavior of Dissipative Systems. - Am. Math. Soc., 1989.

133. Hale J. K., Lin X. B., Raugel G. Upper semicontinuity of attractors for approximations of semigroups and partial differential equations// Math. Comp. - 1988. - 50. - P. 89-123.

134. Han X., Kloeden P. Attractors Under Discretisation. - Springer, 2017.

135. He B., Cao J., Yang B. Weighted Stepanov-like pseudo-almost automorphic mild solutions for semilinear fractional differential equations// Adv. Differ. Eqs. - 2015. - 2015. - 74.

136. Henry D. Geometric Theory of Semilinear Parabolic Equations. - Berlin: Springer-Verlag, 1981.

137. Hess P. Periodic-Parabolic Boundary-Value Problems and Positivity. — Longman, 1991.

138. Hoppe R. H. W. A constructive approach to the Bellman semigroup// Nonlin. Anal. Theory Meth. Appl. - 1985. - 9, № 11. - P. 1165-1181.

139. Huang J., Li J., Shen T. Dynamics of stochastic modified Boussinesq approximation equation driven by fractional Brownian motion// Dynam. Partial Differ. Eqs. — 2014. — 11, № 2. — P. 183-209.

140. Irwin M. C., Smooth Dynamical Systems. - River Edge, New Jersey: World Scientific, 2001.

141. Jin B., Lazarov R., Pasciak J., Zhou Z. Galerkin FEM for fractional order parabolic equations with initial data in $H^{-s}, 0 \leqslant s \leqslant 1 / /$ in: Numerical Analysis and Its Applications, 2013. — P. 24-37.

142. Jin B., Lazarov R., Zhou Z. Error estimates for a semidiscrete finite element method for fractional order parabolic equations// SIAM J. Numer. Anal. — 2013. — 51, № 1. - P. 445-466.

143. Jin B., Li B., Zhou Zh. Discrete maximal regularity of time-stepping schemes for fractional evolution equations// Numer. Math. - 2018. - 138, № 1. - P. 101-131.

144. Kaashoek M. A., Verduyn S. M. An integrability condition on the resolvent for hyperbolicity of the semigroup// J. Differ. Eqs. - 1994. — 112, № 2. - P. 374-406.

145. Kamenskii M., Obukhovskii V., Petrosyan G., Jen-Chih Yao On semilinear fractional-order differential inclusions in Banach spaces// Fixed Point Theory. — 2017. — 18, № 1. — P. 269-292.

146. Kamenskii M., Obukhovskii V., Petrosyan G., Jen-Chih Yao On approximate solutions for a class of semilinear fractional-order differential equations in Banach spaces// Fixed Point Theory Appl. — 2017. -2017 . -28 . 
147. Kamenskii M., Obukhovskii V., Petrosyan G., Jen-Chin Yao Existence and approximation of solutions to nonlocal boundary-value problems for fractional differential inclusions// Fixed Point Theory Appl. 2019. - 2019. - 2 .

148. Kamenskii M., Obukhovskii V., Zecca P. Condensing Multivalued Maps and Semilinear Differential Inclusions in Banach Spaces. — Berlin: Walter de Gruyter, 2001.

149. Karatay I., Bayramoglu S. R. A characteristic difference scheme for time-fractional heat equations based on the Crank-Nicholson difference schemes// Abstr. Appl. Anal. — 2012. — 2012. — 548292.

150. Katayama S. Global Solutions and the Asymptotic Behavior for Nonlinear Wave Equations with Small Initial Data. - Tokyo: Math. Soc. Jpn., 2017.

151. Kato T. Perturbation Theory for Linear Operators. - Berlin: Springer-Verlag, 1995.

152. Keyantuo V., Lizama C., Warma M. Spectral criteria for slvability of boundary value problems and positivity of solutions of time-fractional differential equations// Abstr. Appl. Anal. — 2013. — 2013. 614328.

153. Keyantuo V., Lizama C., Warma M. Existence, regularity, and representation of solutions of time fractional wave equations// Electr. J. Differ. Eqs. - 2017. - 2017. - 222.

154. Keyantuo V., Lizama C., Warma M. Existence, regularity, and representation of solutions of time fractional diffusion equations// Adv. Differ. Eqs. — 2016. — 21, № 9-10. — P. 837-886.

155. Kilbas A. A., Srivastava H. M., Trujillo J. J. Theory and Applications of Fractional Differential Equations. - Amsterdam: Elsevier, 2006.

156. Kloeden P. E. Upper semicontinuity of attractors of delay differential equations in the delay// Bull. Austr. Math. Soc. - 2006. - 73. - P. 299-305.

157. Kloeden P. E., Lorenz J. Stable attracting sets in dynamical system and their one-step discretizations// SIAM J. Numer. Anal. — 1986. — 23. — P. 986-995.

158. Kloeden P. E., Lorenz J. Lyapunov stability and attractors under discretization// Proc. Equadiff 87 Conf. (Xanthi, Greece, August 1987). — Marcel-Dekker, 1989. — P. 361-368.

159. Kloeden P. E., Piskarev S. I. Discrete convergence and the equivalence of equi-attraction and the continuous convergence of attractors// Int. J. Dynam. Syst. Differ. Eqs. - 2007. - 1. - P. 38-43.

160. Kostić M. A note on semilinear fractional equations governed by abstract differential operators// An. Ştiinţ. Univ. ACuza Iaşi 1. I., Ser. Nouă, Mat. — 2016. — 62, № 2. — P. 757-762.

161. Kostin I. N. Lower semicontinuity of a nonhyperbolic attractor// J. London Math. Soc. — 1995 . - 52. - P. 568-582.

162. Kovacs B., Li B., Lubich C. A-Stable time discretizations preserve maximal parabolic regularity// SIAM J. Numer. Anal. — 2016. — 54, № 6. - P. 3600-3624.

163. Ladyzhenskaya O. Attractors for Semigroups and Evolution Equations. - Cambridge: Cambridge Univ. Press, 1991.

164. Larsson $S$. Nonsmooth data error estimates with applications to the study of the long-time behavior of finite element solutions of semilinear parabolic problems. - Chalmers Univ. Technol., 1992.

165. Larsson $S$. Numerical analysis of semilinear parabolic problems// in: The Graduate Student's Guide to Numerical Analysis'98 (Ainsworth M. et al., eds.). — Berlin: Springer-Verlag, 1999. — P. 83-117.

166. Larsson S., Pilyugin S. Yu. Numerical shadowing near the global attractor for a semilinear parabolic equation. - Chalmers Univ. Technol., 1998.

167. Larsson S., Sanz-Serna J. M. The behavior of finite element solutions of semilinear parabolic problems near stationary points// SIAM J. Numer. Anal. — 1994. - 31, № 4. - P. 1000-1018.

168. Larsson S., Sanz-Serna J.-M. A shadowing result with applications to finite element approximation of reaction-diffusion equations// Math. Comp. — 1999. — 68, № 225. - P. 55-72.

169. Latushkin Y., Pogan A., Schnaubelt R. Dichotomy and Fredholm properties of evolution equations// J. Oper. Theory. - 2007. - 58, № 2. - P. 387-414.

170. Latushkin Y., Prüss J., Schnaubelt R. Stable and unstable manifolds for quasilinear parabolic systems with fully nonlinear boundary conditions// J. Evol. Eqs. - 2006. - 6. - P. 537-576.

171. Latushkin Y., Prüss J., Schnaubelt R. Center manifolds and dynamics near equilibria of quasilinear parabolic systems with fully nonlinear boundary conditions// Discr. Cont. Dynam. Syst. Ser. B. — 2008. — 
9. - P. 595-633.

172. Li C., Chen A. Numerical methods for fractional partial differential equations// Int. J. Comp. Math. 2018. — 95, № 6-7. — P. 1048-1099.

173. Li C. Y., Li M. Hölder regularity for abstract fractional Cauchy problems with order in $(0,1) / /$ J. Appl. Math. Phys. - 2018. - 6. - P. 310-319.

174. Li D., Kloeden P. E. Equi-attraction and the continuous dependence of attractors on parameters// Glasgow Math. J. - 2004. - 46. - P. 131-141.

175. Li K., Jia J. Existence and uniqueness of mild solutions for abstract delay fractional differential equations// Comput. Math. Appl. - 2011. — 62, № 3. - P. 1398-1404.

176. Li M., Chen C., Li F. On fractional powers of generators of fractional resolvent families// J. Funct. Anal. - 2010. - 259. - P. 2702-2726.

177. Li M., Morozov V., Piskarev S. On the approximations of derivatives of integrated semigroups// J. Inv. Ill-Posed Probl. - 2010. - 18, № 5. - P. 515-550.

178. Li M., Morozov V., Piskarev S. On approximations of derivatives of integrated semigroups, II// J. Inv. Ill-Posed Probl. - 2011. — 19, № 3-4. - P. 643-688.

179. Li M., Piskarev S. On approximation of integrated semigroups// Taiwan. J. Math. — 2010. — 14, № 6 . - P. 2137-2161.

180. Li M., Zheng Q. On spectral inclusions and approximations of a times resolvent families// Semigroup Forum. - 2004. - 69, № 3. - P. 356-368.

181. Lin K.-B., Liu H., Pang C.-T. Approximate controllability of semilinear functional differential equations with Hille-Yosida operator// J. Nonlin. Convex Anal. — 2016. — 17, № 2. — P. 275-285.

182. Lin $Y$., $X u$ C. Finite difference spectral approximations for the time-fractional diffusion equation// J. Comput. Phys. - 2007. - 225. - P. 1533-1552.

183. Liu F., Wu H. X. Regularity of discrete multisublinear fractional maximal functions// Sci. China Math. - 2017. - 60. - P. 1461-1476.

184. Liu L., Fan Zh., Li G., Piskarev S. Maximal regularity for fractional Cauchy equation in Hölder space and its approximation// Comput. Meth. Appl. Math. — 2019. - 19, № 2. - P. 160-178.

185. Liu H., Chang J. C. Existence for a class of partial differential equations with nonlocal conditions// Nonlin. Anal. Theory Meth. Appl. — 2009. — 70. - P. 3076-3083.

186. Liu R., Li M., Pastor J., Piskarev S. On the approximation of fractional resolution families// Differ. Eqs. - 2014. - 50, № 7. - P. 927-937.

187. Liu R., Li M., Piskarev S. I. Approximation of semilinear fractional Cauchy problem// Comput. Meth. Appl. Math. - 2015. - 15, № 2. - P. 203-212.

188. Liu R., Li M., Piskarev S. I. Stability of difference schemes for fractional equations// Differ. Eqs. - 2015. - 51. - P. 904-924.

189. Liu R., Li M., Piskarev S. I. The order of convergence of difference schemes for fractional equations// Numer. Funct. Anal. Optim. - 2017. - 38. - P. 754-769.

190. Liu R., Piskarev S. Numer. Funct. Anal. Optim. - 2021. - 10.1080/01630563.2021.1901117.

191. Liu R., Piskarev S. The order of convergence of difference schemes for nonhomogeneous fractional equations// Numer. Funct. Anal. Optim..

192. Liu R., Piskarev S. Approximation of semilinear fractional Cauchy problem, II// Semigroup Forum. 2020. - 101, № 3. - P. 751-768.

193. Lizama C. Abstract linear fractional evolution equations// in: Fractional Differential Equations (Kochubei A., Luchko Yu., eds.). - Berlin-Boston: de Gruyter, 2019. — P. 465-498.

194. Lizama C. Abstract linear fractional evolution equations// in: Fractional Differential Equations (Kochubei A., Luchko Yu., eds.). — Berlin-Boston: de Gruyter, 2019. — P. 499-514.

195. Lizama C., Pereira A., Ponce R. On the compactness of fractional resolvent operator functions// Semigroup Forum. - 2016. - 93. - P. 363-374.

196. Lord G. J., Stuart A. M. Discrete Gevrey regularity attractors and upper semicontinuity for a finite difference approximation to the Ginzburg-Landau equation// Numer. Funct. Anal. Optim. — 1995. - 16, 
№ 7-8. - P. 1003-1047.

197. Lorenz T. Differential equations for closed sets in a Banach space, survey and extension// Vietnam J. Math. - 2017. - 45, № 1-2. - P. 5-49.

198. Mainardi F. Fractional diffusive waves in viscoelastic solids// in: Nonlinear Waves in Solids ( Wegner J. L., Norwood F. R., eds.). — Fairfield, 1995. - P. 93-97.

199. Martínez C., Sanz M. The Theory of Fractional Powers of Operators. - Amsterdam: North-Holland, 2001.

200. Matsumoto T., Tanaka N. Abstract Cauchy problem for weakly continuous operators// J. Math. Anal. Appl. - 2016. - 435, № 1. - P. 267-285.

201. Meng F., Liu C., Zhang C. Existence of multiple equilibrium points in global attractor for damped wave equation// Boundary-Value Probl. - 2019. - 2019. - 6.

202. Miller K. S., Ross B. An Introduction to the Fractional Calculus and Fractional Differential Equations. — New York: Wiley, 1993.

203. Miyazaki R., Kim D., Naito T., Jong Son Shin Fredholm operators, evolution semigroups, and periodic solutions of nonlinear periodic systems// J. Differ. Eqs. — 2014. — 257, № 11. — P. 4214-4247.

204. Moshrefi-Torbati M., Hammond J. K. Physical and geometrical interpretation of fractional operators// J. Franklin Inst. — 1998. - 335B, № 6. - P. 1077-1086.

205. Nevanlinna O., Vainikko G. Limit spectrum of discretely converging operators// Numer. Funct. Anal. Optim. - 1996. - 17, № 7-8. - P. 797-808.

206. Olszowy L. Existence of mild solutions for semilinear nonlocal Cauchy problems in separable Banach spaces// Z. Anal. Anwend. — 2013. — 32, № 2. — P. 215-232.

207. Olszowy L. Existence of mild solutions for the semilinear nonlocal problem in Banach spaces// Nonlin. Anal. - 2013. - 81. - P. 211-223.

208. Ostermann A., Palencia C. Shadowing for nonautonomous parabolic problems with applications to longtime error bounds// SIAM J. Numer. Anal. — 2000. — 37, № 5. — P. 1399-1419.

209. Owolabi K. M., Atangana A. Numerical simulations of chaotic and complex spatiotemporal patterns in fractional reaction-diffusion systems// Comput. Appl. Math. - 2018. — 37, № 2. — P. 2166-2189.

210. Owolabi K. M., Atangana A. Robustness of fractional difference schemes via the Caputo subdiffusionreaction equations// Chaos Solitons Fract. — 2018. — 111. — P. 119-127.

211. Palmer K. J. A perturbation theorem for exponential dichotomies// Proc. Roy. Soc. Edinburgh Sec. A 1987. - 106, № 1-2. - P. 25-37.

212. Palmer K. J. Exponential dichotomies and Fredholm operators// Proc. Am. Math. Soc. — 1988. — 104, № 1. - P. 149-156.

213. Palmer K. Shadowing in Dynamical Systems. Theory and Applications. — Dordrecht: Kluwer Academic, 2000.

214. Pastor J., Piskarev S. The exponential dichotomy under discretization on general approximation scheme// Adv. Numer. Anal. — 2011. - 2011. - 582740.

215. Paunonen L. Robustness of strong stability of semigroups// J. Differ. Eqs. — 2014. — 257, № 12 . — P. 4403-4436.

216. Pazy A. Semigroups of Differential Equations. - New York-Basel: Marcel Dekker, 1983.

217. Petryshyn W. V. Approximation-Solvability of Nonlinear Functional and Differential Equations. - New York-Basel-Hong Kong: Marcel Dekker, 1993.

218. Pilyugin S. Yu. Shadowing in Dynamical Systems. — Berlin: Springer-Verlag, 1999.

219. Piskarev S. On approximation of holomorphic semigroups// Tartu Riikl. Ul. Toimetised — 1979 . — 492. - P. 3-23.

220. Piskarev S. I. Estimates for the rate of convergence in the solution of ill-posed problems for evolution equations// Izv. Akad. Nauk SSSR. Ser. Mat. — 1987. — 51, № 3. - P. 676-687.

221. Piskarev S. I. Approximation of positive $C_{0}$-semigroups of operators// Differ. Uravn. — 1991. -27 , № 7 . - P. 1245-1250.

222. Piskarev S. and S. Siegmund Approximations of stable manifolds in the vicinity of hyperbolic equilibrium points for fractional differential equations// Nonlin. Dynam - 19. — 95, № 1. — P. 685-697. 
223. Podlubny I. Fractional Differential Equations. - Academic Press, 1998.

224. Ponce R. Existence of mild solutions to nonlocal fractional Cauchy problems via compactness// Abstr. Appl. Anal. — 2016. - 2016. - 4567092.

225. Poongodi R., Murugesu R. Existence of mild solution for fractional nonlocal neutral impulsive integrodifferential equations with state-dependent delay// Nonlin. Stud. — 2016. — 23, № 2. — P. 209-223.

226. Poongodi R., Murugesu R., Nirmalkumar R. Exact controllability results for a class of abstract nonlocal Cauchy problem with impulsive conditions// Evol. Equ. Control Theory. — 2017. — 6, № 4. — P. 599-613.

227. Prüss J. Evolutionary Integral Equations and Applications. — Basel: Birkhäuser, 1993.

228. Ran $Y$., Li J. Pullback attractors for non-autonomous reaction-diffusion equation with infinite delays in $C_{\gamma, L^{r}(\Omega)}$ or $C_{\gamma, W^{1, r}(\Omega)} / /$ Boundary Value Probl. - 2018. - 2018. - 99.

229. Rau R. T. Hyperbolic evolution groups and dichotomic evolution families// J. Dynam. Differ. Eqs. 1994. 一 6, № 2. - P. 335-350.

230. Rau R. T. Hyperbolic evolution semigroups on vector valued function spaces// Semigroup Forum. - 1994. — 48, № 1. - P. 107-118.

231. Robinson J. C. Infinite-Dimensional Dynamical Systems. An Introduction to Dissipative Parabolic PDEs and the Theory of Global Attractors. - Cambridge: Cambridge Univ. Press, 2001.

232. Robinson R. C. An Introduction to Dynamical Systems: Continuous and Discrete. - Upper Saddle River, New Jersey: Pearson Prentice Hall, 2004.

233. Rottmann J. Spectral Properties of Mixed Hyperbolic-Parabolic Systems. — Univ. Bielefeld, 2005.

234. Sano H., Tanaka N. Well-posedness and flow invariance for semilinear functional differential equations governed by nondensely defined operators// Differ. Integral Eqs. — 2017. — 30, № 9-10. — P. 695-734.

235. Sayevand K., Pichaghchi K. Successive approximation: a survey on stable manifold of fractional differential systems// Fract. Calc. Appl. Anal. - 2015. - 18. - P. 621-641.

236. Sell G. R., You Y. Dynamics of Evolutionary Equations. - New York: Springer-Verlag, 2002.

237. Shomberg J. L. Attractors for damped semilinear wave equations with singularly perturbed acoustic boundary conditions// Electron. J. Differ. Eqs. — 2018. — № 152.

238. Siegmund S., Piskarev S. Approximations of stable manifolds in the vicinity of hyperbolic equilibrium points for fractional differential equations// Nonlin. Dynam. - 2019. — 95, № 1. - P. 685-697.

239. Smale S. Differentiable dynamical systems// Bull. Am. Math. Soc. — 1967. — 73. — P. 747-817.

240. Sobolevskii P. E. The coercive solvability of difference equations// Dokl. Akad. Nauk SSSR. - 1971. 201. - P. 1063-1066.

241. Stuart A. Perturbation theory for infinite-dimensional dynamical systems// Proc. Sixth SERC Summer School in Numerical Analysis (Leicester, UK, July 18-29, 1994). — Oxford: Clarendon Press, 1995. P. 181-290.

242. Stuart A. Convergence and stability in the numerical approximation of dynamical systems// in: The State of the Art in Numerical Analysis. - New York: Oxford Univ. Press, 1997. — P. 145-169.

243. Stuart A. M., Humphries A. R. Numerical Analysis and Dynamical Systems. - Cambridge: Cambridge Univ. Press, 1996.

244. Stummel F. Diskrete Konvergenz linearer Operatoren// Proc. Conf. "Linear Operators and Approximation" (Oberwolfach Math. Res. Inst., 1971). — Basel: Birkhäuser, 1972. — P. 196-216.

245. Stummel F. Perturbation of domains in elliptic boundary value problems// Lect. Notes Math. — 1976. 503. - P. 110-136.

246. Tatar N. The decay rate for a fractional differential equation// J. Math. Anal. Appl. — 2004. — 295. P. 303-314.

247. Tateishi A. A., Ribeiro H. V., Lenzi E. K. The role of fractional time-derivative operators on anomalous diffusion// Front. Phys. - 2017. - 5. - 52.

248. Thomée V. Finite element methods for parabolic problems. Some steps in the evolution// in: Finite Element Methods. — New York: Marcel Dekker, 1994. — P. 433-442.

249. Tomilov Yu. A resolvent approach to stability of operator semigroups// J. Operator Theory. — 2001. 46. - P. 63-98. 
250. Tran Dinh Ke and Tran Van Tuan Finite-time attractivity for semilinear fractional differential equations// Res. Math. - 2018. - 73, № 7. - P. 1-19.

251. Trotter H. F. Approximation of semigroups of operators// Pac. J. Math. — 1958. — 8. - P. 887-919.

252. Trotter H. F. Approximation and perturbation of semigroups// Proc. Conf. "Linear Operators and Approximation" (Oberwolfach Math. Res. Inst., 1974). — Basel: Birkhäuser, 1974. — P. 3-21.

253. Tuan Hoang The, Siegmund S., Son Doan Thai, Cong Nguyen An instability theorem for nonlinear fractional differential systems// Discr. Contin. Dynam. Syst. Ser. B. — 2017. — 22, № 8. — P. 3079-3090.

254. Uchaikin V. V. Fractional Derivatives for Physicists and Engineers. - Berlin-Heidelberg: Springer-Verlag, 2013.

255. Ushijima T. Approximation theory for semigroups of linear operators and its application to approximation of wave equations// Jpn. J. Math. — 1975. — 1, № 1. - P. 185-224.

256. Vainikko G. Funktionalanalysis der Diskretisierungsmethoden. — Leipzig: Teubner Verlag B.G., 1976.

257. Vainikko G. Über die Konvergenz und Divergenz von Näherungsmethoden bei Eigenwertproblemen// Math. Nachr. - 1977. - 78. - P. 145-164.

258. Vainikko G. Über Konvergenzbegriffe für lineare Operatoren in der numerischen Mathematik// Math. Nachr. - 1977. - 78. - P. 165-183.

259. Vainikko G. Approximative methods for nonlinear equations (two approaches to the convergence problem)// Nonlin. Anal. Theory Meth. Appl. — 1978. — 2. — P. 647-687.

260. Vainikko G. Foundations of finite difference method for eigenvalue problems// Proc. Summer School "The Use of Finite Element Method and Finite Difference Method in Geophysics" (Liblice, 1977). — Prague: Česk. Akad. Věd, 1978. - P. 173-192.

261. Vainikko G., Piskarev S. Regularly compatible operators// Izv. Vyssh. Ucheb. Zaved. Mat. — 1977. — № 10. - P. 25-36.

262. Vasiliev V. V., Piskarev S. I. Differential equations in Banach spaces. II. Cosine-operator functions// J. Math. Sci. - 2004. — 122, № 2. - P. 3055-3174.

263. Vijayakumar V., Murugesu R., Poongodi R., Dhanalakshmi S. Controllability of second-order impulsive nonlocal Cauchy problem via measure of noncompactness// Mediterr. J. Math. — 2017. — 14, № 1 . — 3 .

264. Voigt J. On the convex compactness property for the strong operator topology// Note Mat. — 1992. — 12. - P. 259-269.

265. Vrabie I. I. A class of semilinear delay differential equations with nonlocal initial conditions// Dynam. Partial Differ. Eqs. - 2018. - 15, № 1. - P. 45-60.

266. Vu Quoc Phong A new proof and generalizations of Gearhart's theorem// Proc. Am. Math. Soc. — 2007. - 135, № 7. - P. 2065-2072.

267. Vu Quoc Phong The spectral radius, hyperbolic operators and Lyapunov's theorem// in: Evolution Equations and Their Applications in Physical and Life Sciences. — New York: Marcel Dekker, 2001. — P. 187194.

268. Vu Quoc Phong On the exponential stability and dichotomy of $C_{0}$-semigroups// Stud. Math. — 1999. 132, № 2. - P. 141-149.

269. Vu Quoc Phong, Schuler E. The operator equation $A X-X B=C$, admissibility, and asymptotic behavior of differential equations// J. Differ. Eqs. - 1998. - 145, № 2. - P. 394-419.

270. Wang R. N., Chen D. H., Xiao T. J. Abstract fractional Cauchy problems with almost sectorial operators// J. Differ. Eqs. - 2012. — 252. - P. 202-235.

271. Wang R. N., Xiao T. J., Liang J. A note on the fractional Cauchy problems with nonlocal initial conditions // Appl. Math. Lett. - 2011. - 24. — P. 1435-1442.

272. Xia Zh., Wang D., Ching-Feng Wen, and Jen-Chih Yao Pseudo asymptotically periodic mild solutions of semilinear functional integro-differential equations in Banach spaces// Math. Meth. Appl. Sci. — 2017. — 40, № 18. - P. 7333-7355.

273. Xiao J.-Zh., Wang Zh.-Y., Liu J. Hausdorff product measures and $C^{1}$-solution sets of abstract semilinear functional differential inclusions// Topol. Meth. Nonlin. Anal. — 2017. — 49, № 1. — P. 273-298.

274. Yosida K. Functional Analysis. — Berlin: Springer-Verlag, 1965. 
275. Yu J., Wang $Z$. Existence of mild solutions for nonlocal impulsive evolution equations with noncompact semigroups// J. Nanchang Univ. Nat. Sci. — 2016. — 40, № 4. — P. 319-323.

276. Zhang H., Zhang W., Hu Q. Global existence and blow-up of solution for the semilinear wave equation with interior and boundary source terms// Boundary-Value Probl. — 2019. — 2019. — 18.

277. Zhang J., Zhong C., You B. The existence of multiple equilibrium points in global attractors for some symmetric dynamical systems, II// Nonlin. Anal. Real World Appl. — 2017. — 36. — P. 44-55.

278. Zhang Q., Li G. Global attractors of strongly damped wave equations with critical nonlinearities// Acta Math. Appl. Sin. - 2017. — 40, № 2. - P. 192-203.

279. Zhao X. Fourier spectral approximation to global attractor for $2 \mathrm{D}$ convective Cahn-Hilliard equation// Bull. Malays. Math. Sci. Soc. (2). — 2018. — 41, № 2. — P. 1119-1138.

Пискарев Сергей Игоревич

Научно-исследовательский вычислительный центр МГУ им. М. В.Ломоносова;

Марийский государственный университет, Йошкар-Ола;

Всероссийский институт научной и технической информации РАН, Москва

E-mail: piskarev@gmail.com

Овчинников Алексей Витальевич

МГУ им. М. В.Ломоносова;

Всероссийский институт научной и технической информации РАН, Москва

E-mail: ovchinnikov@viniti.ru 